Florida International University FIU Digital Commons

7-6-2016

\title{
Doping as a Possible Means to create Superconductivity in Graphene
}

Kiar Holland

Florida International University, kholl004@fiu.edu

DOI: 10.25148 /etd.FIDC000772

Follow this and additional works at: https://digitalcommons.fiu.edu/etd

\section{Recommended Citation}

Holland, Kiar, "Doping as a Possible Means to create Superconductivity in Graphene" (2016). FIU Electronic Theses and Dissertations. 2550.

https://digitalcommons.fiu.edu/etd/2550

This work is brought to you for free and open access by the University Graduate School at FIU Digital Commons. It has been accepted for inclusion in FIU Electronic Theses and Dissertations by an authorized administrator of FIU Digital Commons. For more information, please contact dcc@fiu.edu. 


\title{
FLORIDA INTERNATIONAL UNIVERSITY
}

Miami, Florida

\section{DOPING AS A POSSIBLE MEANS TO CREATE SUPERCONDUCTIVITY IN GRAPHENE}

\author{
A dissertation submitted in partial fulfillment of \\ the requirements for the degree of \\ DOCTOR OF PHILOSOPHY \\ in \\ ELECTRICAL ENGINEERING \\ by
}

Kiar J. Holland

2016 
To: Interim Dean Ranu Jung

College of Engineering and Computing

This dissertation, written by Kiar J. Holland, and entitled Doping as a Possible Means to Create Superconductivity in Graphene, having been approved in respect to style and intellectual content, is referred to you for judgment.

We have read this dissertation and recommend that it be approved.

Jeffrey Fan

Armando Barreto

Kinzy Jones

Chunlei Wang

Grover Larkins, Major Professor

Date of Defense: July 6, 2016

The dissertation of Kiar J. Holland is approved.

Interim Dean Ranu Jung College of Engineering and Computing

Andrés G. Gil

Vice President for Research and Economic Development and Dean of the University Graduate School

Florida International University, 2016 


\section{ABSTRACT OF THE DISSERTATION}

\section{DOPING AS A POSSIBLE MEANS TO CREATE SUPERCONDUCTIVITY IN}

\section{GRAPHENE}

by

Kiar J. Holland

Florida International University, 2016

Miami, Florida

\section{Professor Grover Larkins, Major Professor}

The possibility of creating superconductivity in Highly Oriented Pyrolytic Graphite (HOPG) by means of doping was investigated. Bulk HOPG samples were doped with phosphorous using either ion-implantation or by Chemical Vapor Deposition growth with phosphine in the gas mixture. The methods for testing the graphene samples, once doped, were done by performing $R$ vs. $T$ measurements, and determining via observation suppressed superconductive characteristics signaling the presence of the Meissner Effect in a strong applied magnetic field. Before doping, the resistance vs. temperature ( $R$ vs. $T$ ) characteristic of the HOPG was measured. The $R$ vs. $T$ characteristic was again measured after doping, and for surface multilayers of graphene exfoliated from the post doped bulk sample. A 100 to $350 \mathrm{mT}$ magnetic field was supplied, and the $R$ vs. $T$ characteristic was re-measured on a number of samples.

Phosphorous-implanted HOPG samples exhibit deviations from the expected rise in resistance as the temperature is reduced to some point above $100 \mathrm{~K}$. The application of a modest magnetic field reverses this trend. A step in resistance at a temperature of approximately $50-60 \mathrm{~K}$ in all of the samples is clearly observed, as well as a second step 
at $100-120 \mathrm{~K}$, a third at a temperature range of $150-180 \mathrm{~K}$ and a fourth from about 200$240 \mathrm{~K}$. A response consistent with the presence of magnetic field flux pancake vortices in phosphorous implanted HOPG and in phosphorous-doped exfoliated multilayer graphene has been observed. The lack of zero resistance at low temperatures is also consistent with pancake vortex behaviour in the flux-flow regime. The presence of magnetic vortices requires, and is direct evidence of superconductivity. 


\section{TABLE OF CONTENTS}

CHAPTER

PAGE

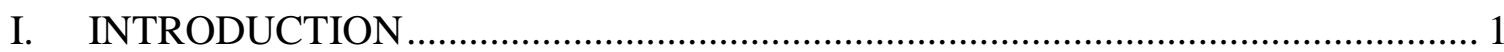

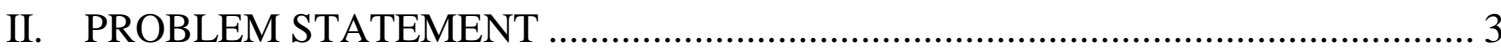

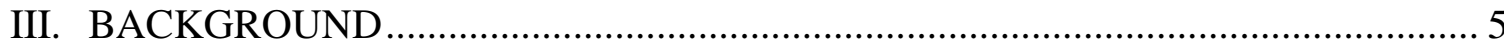

A. Highly Oriented Pyrolytic Graphite (HOPG) ……............................................ 5

B. Type 1 Superconductivity ...................................................................... 7

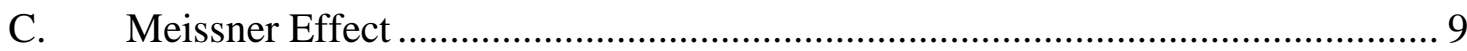

D. Inherent Superconductive Traits ............................................................. 9

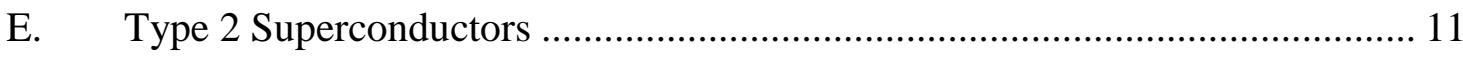

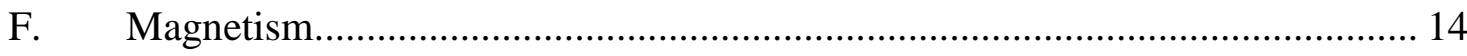

G. Resistance versus Temperature ................................................................... 18

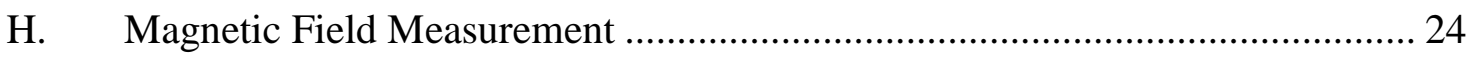

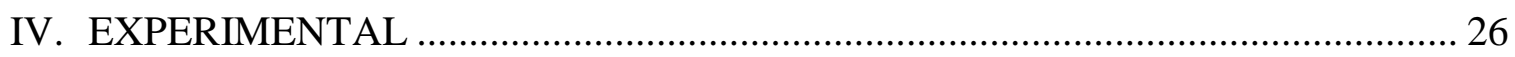

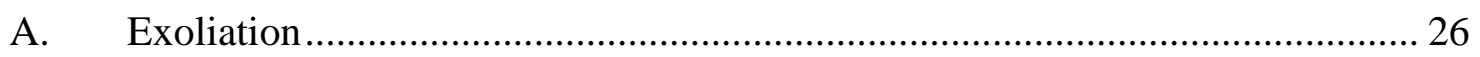

B. Resistance versus Temperature Measurement ................................................... 31

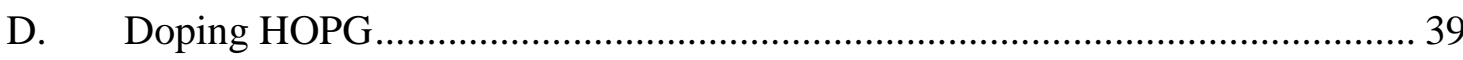

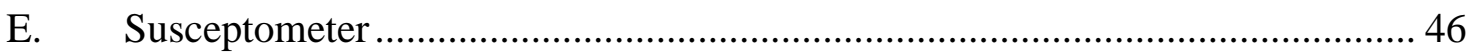

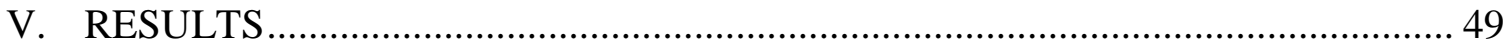

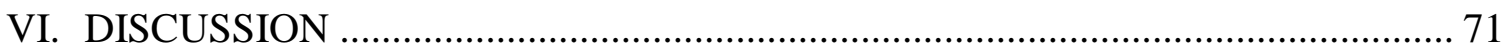

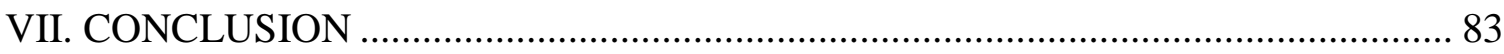

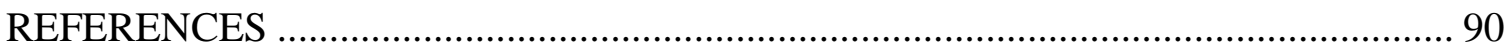

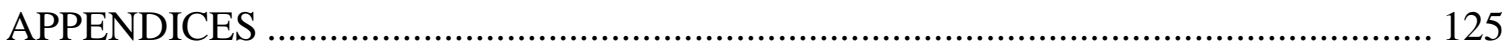

VITA ……… 


\section{TABLE OF TABLES}

TABLE

PAGE

Table 1. HOPG Grades. Data Collected and Confirmed from [79]-[84] ........................ 6

Table 2. Type 1 Superconductors. Data from [166] .............................................. 8

Table 3. Solvents tested for graphene lift off from Kapton ${ }^{\circledR}$ vacuum tape and general purpose Scotch ${ }^{\circledR}$ tape. The green shade denotes lift off while still floating in the solvent. Yellow shading denotes lift off with the aid of tweezers. Red denotes no lift

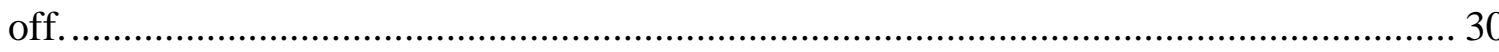

Table 4. Stopping and range for ion-implanted species at $10 \mathrm{keV}$ and $5 \mathrm{keV}$ for argon. Green highlights the species that showed features that warrant further investigation...... 41

Table 5. Dose and concentration for ion-implanted species. All were done at $10 \mathrm{keV}$ with the exception of argon which was done at $5 \mathrm{keV}$. All used a current of $104 \mathrm{pA}$. Green highlights the species that showed features that warrant further investigation...... 42

Table 6. Multi-energy implation of phosphorus for sample 065 ................................ 43

Table 7. Plasma Enhanced CVD recipe. Phosphine used was 1000 ppm in argon. ....... 43 


\section{TABLE OF FIGURES}

FIGURE

PAGE

Figure 1: A HOPG sample with scratch used to indicate the bottom side....................... 7

Figure 2: Four probe measurement. Collinear setup. Picture courtesy of [89]............... 20

Figure 3: Correction factor plot. Picture courtesy of [89]..................................... 21

Figure 4: Delta Mode reading calculation. Screenshot of Keithley's help menu in test

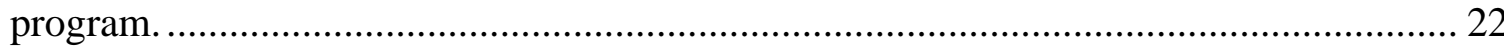

Figure 5: Delta Mode Cancelling Thermoelectric drift. ........................................... 23

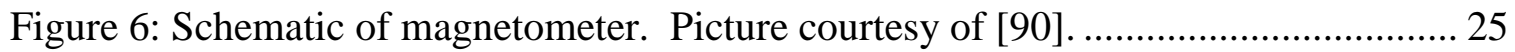

Figure 7: System created for sample preparation. Chuck with through hole to create vacuum, capable of holding down HOPG sample. Silicon Kapton ${ }^{\circledR}$ tape was added to give a soft surface for the HOPG to rest, which was found to give a better vacuum seal. Vacuum system with tubes connected to chuck shown on the left.

Figure 8: Sample preparation system with HOPG sample secured to chuck via vacuum seal. A piece of Kapton ${ }^{\circledR}$ tape will be laid on top of the HOPG, and carefully pressed down to remove all air bubbles.

Figure 9: Keithley $6221 \mathrm{DC}$ and AC current source (on left) and Keithley 2182A nanovoltmeter (on right) with external cooling fan in between to keep airflow constant for cooling of the device.

Figure 10: The peeled sample lifted from the HOPG, loaded into the cryogenic system where probes are placed collinearly onto a continuous area of graphene.

Figure 11: Colinear Probes positioned on sample with broken pieces of Lanthanum Aluminate ( $\mathrm{LaAl})$ placed near the edges to prevent curling.

Figure 12: Coil created in-house to generate magnetic field. This slides over the outer shield and was adjusted to make the center of the coils on the same plane as the sample. The ends of the copper coils are connected to a 4 amp DC source.

Figure 13: Flow chart for $R$ vs. $T$ measurement subVI for data acquisition. 37

Figure 14: Flow chart for $R$ vs. $T$ measurement subVI for data acquisition continued.... 38 
Figure 15: SRIM simulated distribution of (1) phosphorus ions implanted in graphite at $E p=10 \mathrm{keV}$, (2) damage in graphite lattice caused by implant and (3) probable region of effect.

Figure 16: Copper witness visually showing graphene after successful Chemical Vapor Deposition process.

Figure 17: Graphite sample holder with array of HOPG samples loaded. Two copper witness samples are placed at the upper left corner of the array to indicate the position of the first sample in the series.

Figure 18: Susceptometer System. 46

Figure 19: Outer coil, inner coil and 2 pieces of single bore alumina. 47

Figure 20: Brass guides, custom made to center coils. These are screwed on top of the coldhead.

Figure 21: Outer coil, inner coil, alumina with sample between alumina pieces and centered using custom sample holder are placed on top of the coldhead and held in placed by 4 brass guides that wedge the assembly in place.

Figure 22: Measured $R$ vs. $T$ dependence of a phosphorous-implanted HOPG sample. Curve 1 (black) is before implantation, curve 2 (red) is of the phosphorous-implanted sample before magnetic field was applied, curve 3 (green) is of the phosphorousimplanted sample with magnetic field applied and curve 4 (blue) is of the phosphorous-implanted sample after the magnetic field applied in curve 3 magnetic field was removed.

Figure 23: $R$ vs. $T$ of thin film exfoliated from phosphorous-doped HOPG measured without (1) and with $0.035 \mathrm{~T}$ applied magnetic field (2) ....................................... 51

Figure 24. Aluminum ion-implanted sample. Bulk HOPG with low dose..................... 52

Figure 25. Arsenic ion-implanted sample. Peel with low dose..................................... 52

Figure 26. Beryllium ion-implanted sample. Bulk with low dose.............................. 53

Figure 27. Fluorine ion-implanted sample. Bulk with low dose............................... 53

Figure 28. Lithium ion-implanted sample. Bulk with low dose. ................................ 54

Figure 29. Nitrogen ion-implanted sample. Bulk with low dose................................ 54

Figure 30. Sodium ion-implanted sample. Bulk with low dose.................................. 55 
Figure 31. Sulfur ion-implanted sample. Bulk with low dose.

Figure 32: Measured $R$ vs. $T$ of four thin films exfoliated from phosphorousimplanted (Ep $=10 \mathrm{keV}$, dose $1.2 \times 108 \mathrm{~cm}-2)$ HOPG samples. (a) HOPG-008, layer 3; (b) HOPG-019, layer 3; (c) HOPG-019, layer 6; (d) HOPG-021, layer 7. The layer numbers indicate the number of multilayers peeled from the host sample, i.e. layer 7 would be the $7^{\text {th }}$ layer exfoliated from that sample.

Figure 33: This represents the simulated normalized distributions of implanted atoms and lattice damage caused by implanted atoms versus depth in HOPG. This represents the simulated normalized distributions of implanted atoms and lattice damage caused by implanted atoms versus depth in HOPG. Curve 1 is the calculated distribution of the implanted phosphorous, curve 2 is the computed damage caused by the implanted phosphorous, curve 3 is the calculated distribution of the implanted argon and curve 4 is the computed damage caused by the implanted argon.

Figure 34: $R$ vs. $T$ of a thin film sample peeled off phosphorous-implanted and then argon-implanted bulk HOPG-023. Curves 1 to 4 are four identical sequential runs with the same probe position.

Figure 35. Multi energy implantation Sample 065.

Figure 36. PECVD graphene grown in the presence of phosphine. First peel taken from bulk HOPG.

Figure 37: Magnetization measurements: Zero-Field Cooled (ZFC) and Field Cooled (FC) $\mathrm{M} / \mathrm{H}$ plots along with a $1 \mathrm{~T}$ Field Cooled $\mathrm{M} / \mathrm{H}$ plot for a film exfoliated from a doped-while-grown Plasma Chemical Vapor Deposition sample.

Figure 38: The free energy (a-c) and magnetization (d-f) as a function of the applied magnetic field for a filled square (a,d) and for the square with two (b,e) and four (c,f) anti-dots. The insets show the free energy for higher vorticity. The vertical lines show the ground state transitions between different vortex states and open circles indicate continuous transitions between different vortex states. The GL parameter is $k=0.28$ [277].

Figure 39: Phosphorous-doped during growth in plasma Chemical Vapor Deposition graphene sample zero-field-cooled magnetization results plotted on same axis type as used in Berdiyorov's work. Note same general shape as in Figure 38(d).

Figure 40: AC Susceptometer results for a graphene film on Kapton ${ }^{\circledR}$ tape exfoliated from a phosphorous-doped-while-grown Chemical Vapor Deposition sample. (a) film was cut into multiple pieces and the pieces were stacked to increase the screening fraction; (b) magnified vertical axis AC susceptometer results for the same sample and run as shown in (a). 
Figure 41: AC Susceptometer results for a $\mathrm{Pr} 2-\mathrm{xCexCuO} 4$ superconductor in the same instrument as was used to produce the data shown in Figure 40. The magnified transition region is shown in the inset.

Figure 42: Raman spectra for a film on Kapton ${ }^{\circledR}$ tape exfoliated from a phosphorousdoped-while-grown Chemical Vapor Deposition graphene sample. The peak ratios give a thickness of approximately 5 monoatomic layers.

Figure 43: Hall effect at $\mathrm{T}=293 \mathrm{~K}$ and $\mathrm{T}=80 \mathrm{~K}$ for the same film on Kapton ${ }^{\circledR}$ tape exfoliated from a phosphorous doped-while-grown Chemical Vapor Deposition graphene sample as is shown in the Raman spectrum in Figure 42.

Figure 44: Graph of the four point resistance (averaged over all contact configurations) of the device at room temperature and in 0T field. Vxx preamplifier reversed the polarity. 4 terminal resistance of the film determined to be $45 \mathrm{mOhm}$.

Figure 45: Graph of the Hall signal after symmetrization the different voltage probe and current configurations were made symmetric at room temperature.

Figure 46: Graph of the Hall signal after symmetrization the different voltage probe and current configurations were made symmetric at $80 \mathrm{~K}$. 


\section{ABBREVIATIONS AND ACRONYMS}

Highly Oriented Pyrolytic Graphite HOPG

Yttrium Barium Copper Oxide

$\mathrm{YBa}_{2} \mathrm{Cu}_{3} \mathrm{O}_{7-\mathrm{X}}$

Super Conducting Quantum Interference Device

SQUID 


\section{INTRODUCTION}

Electrical resistance causes a loss of power from point $\mathrm{A}$ to point $\mathrm{B}$, due to the effects of Joule heating [1]. This conversion of electrical power leads to heat dissipation. As the temperature increases, the electrical resistance increases further [2]-[14], which can make the device unreliable, inefficient, slow, and increases the probability of premature failure [15]-[41]. In order to offset electrical (resistive) losses in power transmission, utility companies generate more power than is delivered to the end user [1].

Superconductivity is a phenomenon that typically appears at very low temperatures. A superconductor exhibits zero resistance while also expelling a magnetic field [42]. A room temperature superconductive material could facilitate the implementation of many power intensive systems (in MRIs etc.) [43], [44].

A room temperature superconductor would revolutionize technology; not only in the field of electrical and electronic engineering; but also in the extended capabilities attainable and new levels of freedom in the design and uses of electronic devices. Computers would work faster, cooler and more reliably [15]-[41]. Mobile devices would have increased battery life. The cost of electricity generation and transmission would lower, and the list goes on [1].

To date, there have not been any room temperature superconducting materials found [45]-[52]. This is why the search for new types of superconductors with higher critical temperatures is critical.

Carbon is known to possess a large number of allotropes, exhibiting interesting and unique mechanical, chemical and electrical properties [91]. Recently, quite a number of 
researchers have turned their attention to graphene [92]-[108], [162], a two dimensional carbon structure that could be used as a test object to study properties necessary for superconductivity [161], [163].

An economical method of obtaining graphene was discovered by Andre Geim and Konstantin Novoselov in 2004 when they pulled ordinary adhesive tape off the surface of graphite [53], [54], producing a monolayer of carbon atoms adhered to the tape. Through their work, they discovered that this single carbon layer was not only the thinnest known material, but also the strongest [53], [54], [111]. The high conductivity and translucent properties of graphene sparked the interest of the photovoltaic industry and touchscreen manufactures, among many other groups. Geim and Novoselov went on to win the Nobel Prize in Physics in 2010 for their work [53], [54].

Doping graphene, due to its close coupling or strong scattering by plasmons as well as phonons has led many to the belief that the Critical Temperature of a superconductor can be raised higher than previously observed in other materials [72]-[78]. More specifically, it has also been suggested that this can be done by raising the density of conduction electrons for each graphene sheet [58]-[66].

Highly Oriented Pyrolytic Graphite (HOPG) was used in preliminary studies of this work, as interlayer coupling in it is weak [109], [110]. This makes it provide a good approximation for graphene. The robust mechanical nature of HOPG, along with its ease of use made it the preferred starting material. An electrical response suggestive of superconductivity in phosphorous (electron donor) implanted Highly Oriented Pyrolytic Graphite was observed. The ultimate critical temperature in this system appeared to be in excess of $100 \mathrm{~K}$. It was noted that the critical temperature may be considerably higher if 
damage incurred during the doping process was minimized. This further positioned HOPG as the starting material of choice.

The phenomenon described in this work strongly suggests the presence of superconductivity, and as a result, the decision was made to proceed based on the possibility that superconductivity is present. However, it should be noted that it is not possible to rule out some new and previously undiscovered physical manifestation of the extreme anisotropy and two dimensionality of the material. Due to the results of this work, no viable, established alternative to superconductivity can be proposed at this time.

\section{PROBLEM STATEMENT}

Prior to our work, there has never, to our knowledge, been an exhaustive, systematic attempt to dope graphene to create a high temperature superconductor. Phosphorus doped graphene created by Plasma Enhanced Chemical Vapor Deposition has not been created or studied and should have unique characteristics. A safe, custom system, built in-house, will need to be created to achieve phosphine Plasma Enhanced Chemical Vapor Deposition. We will need to build a custom cryogenic system for measuring graphene and HOPG at near absolute zero while also going to high temperatures so that we do not miss an above room temperature transition. A magnetic field generator will need to be built for the system so that the sample being tested is not disturbed when the physical apparatus is applied. We will need to develop custom software to orchestrate automation from highly accurate voltage measurements, control current generation, compressor, temperature monitoring and temperature controller. A magnetic susceptometer will need to be constructed, capable of near absolute zero 
temperatures while also being sensitive enough to detect slight changes in the magnetic field of a thin film.

Hypothesis:

- Based on our previous work of doping HOPG with boron and phosphorus dopants, we determined that doping using phosphorus and other electron donors could create a high temperature superconductor.

- Graphene doped with electron donors should create flux vortices and become a type II superconductor.

- Graphene doped using plasma enhanced Chemical Vapor Deposition will minimize damage to the lattice and allow longer coherence lengths. This will improve its superconductivity but create flux flow which will add to the resistance.

- Multilayer graphene doped using ion implantation will create columnar defects that will pin the flux vortices and prevent them from moving. This will create pancake vortices and lower the resistance when the graphene is superconducting.

- The mixed state region will have a very wide transition phase since the anisotropy of graphene is much higher than that of known type II superconductors.

- We expect to see a Hall Effect sign reversal since similar type II superconductors see this. 


\section{BACKGROUND}

In doped graphene, it has previously been hypothesized [72]-[74], [76] that the close coupling or strong scattering of electrons by both phonons and plasmons indicates a potential for superconductivity at considerably higher temperatures than previously observed [112]-[136]. Kopelevich et al. [57]-[67] reported a few cases of suspected superconductivity in Highly Oriented Pyrolytic Graphite, however the results were inconclusive.

As far as it is known, our prior work [72] is the first in which a systematic attempt was made to substitutionally dope HOPG/graphene/graphite into a superconductive state. Later work by Scheike et. al. [140]-[159] and Ballestar [160] also provided hints of possible superconductivity in doped graphite. The work described herein details the efforts in attempting to confirm or disprove these results as originating from superconductivity.

\section{A. Highly Oriented Pyrolytic Graphite (HOPG)}

The focus on the use Highly Oriented Pyrolytic Graphite (HOPG) was due to the difficulties involved with doping graphene, and concerns with how to physically handle graphene in a testing environment without damaging samples. HOPG is easily handled and is structurally a "stack" of graphene sheets [55].

HOPG is available in four different grades of quality; with ZYA being the highest and ZYH being the lowest [79]-[82]. The lower the mosaic spread, the lower the angle of deviation of the grain boundaries from the perpendicular axis and hence, a more highly 
ordered HOPG sample [83], [84]. Thi means that there will also be a larger grain size for higher order HOPG as well [84].

Table 1. HOPG Grades. Data Collected and Confirmed from [79]-[84].

\begin{tabular}{|c|c|c|c|}
\hline \multirow{2}{*}{ Grade } & \multicolumn{2}{|c|}{ Mosaic Spread } & \multirow{2}{*}{ Chip size, $\mathrm{mm}$} \\
\hline & Value & Accuracy & \\
\hline ZYA & $0.4^{\circ}$ & $\pm 0.1^{\circ}$ & \multirow{4}{*}{$10 \times 10$} \\
\hline ZYB & $0.8^{\circ}$ & $\pm 0.2^{\circ}$ & \\
\hline ZYD & $1.5^{\circ}$ & $\pm 0.3^{\circ}$ & \\
\hline $\mathrm{ZYH}$ & $3.5^{\circ}$ & $\pm 0.5^{\circ}$ & \\
\hline
\end{tabular}

While this would affect doping, using lower quality- lower cost ZYH grades for early trials of different doping techniques is more economical. Higher grade HOPG can be used for techniques that prove to be promising in the lower grade $\mathrm{ZYH}$. Furthermore, a slightly less oriented HOPG sample may have beneficial doping properties due to easier implantation in defect areas. 


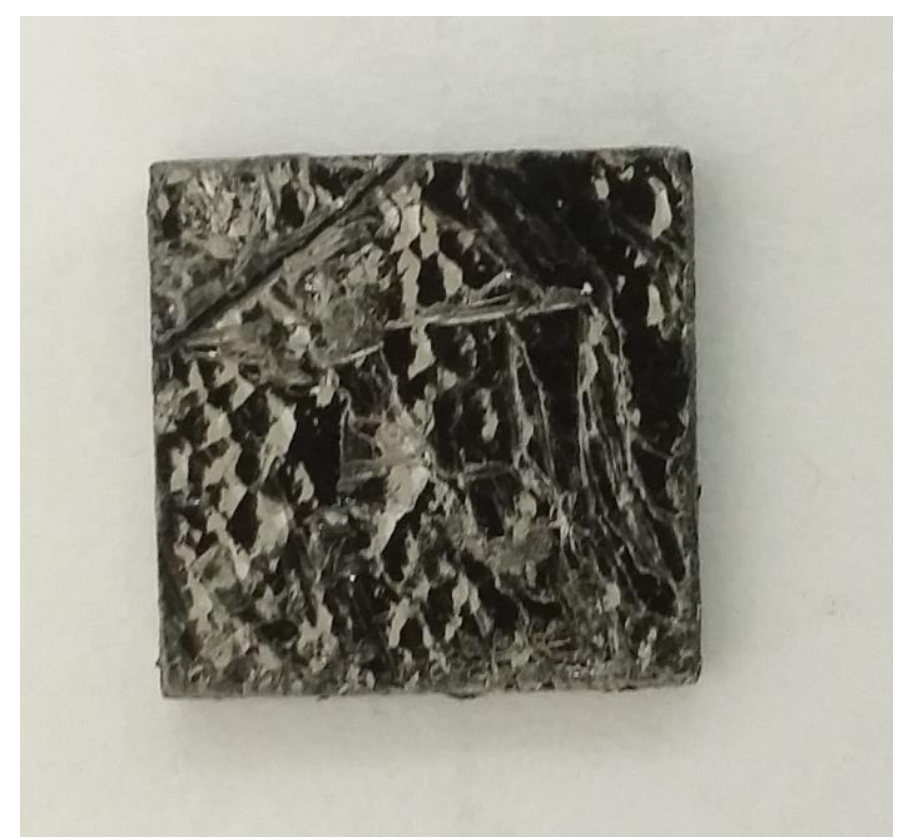

Figure 1: A HOPG sample with scratch used to indicate the bottom side.

\section{B. Type 1 Superconductivity}

On July 10, 1908, helium was successfully liquefied by a Dutch physicist named Heike Kamerling Onnes by using several precooling stages as well as the HampsonLinde cycle [164]. This discovery allowed for testing temperatures that could not be achieved before. By reducing the pressure, he was able to go below helium's boiling point of 4.2 K for a final temperature of $1.5 \mathrm{~K}$ [164],[165]. Three years later in 1911, he found that solid mercury wire submerged in liquid helium had a resistance that abruptly dropped to zero at $4.2 \mathrm{~K}$ [164],[165]. This was the first discovery of superconductivity [164],[165]. Later, many other single element type I superconductors were discovered, as seen in Table 2. 
Table 2. Type 1 Superconductors. Data from [166].

\begin{tabular}{|l|l|c|}
\hline \multicolumn{1}{|c|}{ Material } & Tc (K) & $\begin{array}{c}\text { Lattice } \\
\text { Structure }\end{array}$ \\
\hline Lead (Pb) & 7.196 & FCC \\
\hline Lanthanum (La) & 4.88 & HEX \\
\hline Tantalum (Ta) & 4.47 & BCC \\
\hline Mercury (Hg) & 4.15 & RHL \\
\hline Tin (Sn) & 3.72 & TET \\
\hline Indium (In) & 3.41 & TET \\
\hline Palladium (Pd) & 3.3 & - \\
\hline Chromium (Cr) & 3 & - \\
\hline Thallium (Tl) & 2.38 & HEX \\
\hline Rhenium (Re) & 1.697 & HEX \\
\hline Protactinium (Pa) & 1.40 & TET \\
\hline Thorium (Th) & 1.38 & FCC \\
\hline Aluminum (Al) & 1.175 & FCC \\
\hline Gallium (Ga) & 1.083 & ORC \\
\hline Molybdenum (Mo) & 0.915 & BCC \\
\hline
\end{tabular}

\begin{tabular}{|l|l|c|}
\hline \multicolumn{1}{|c|}{ Material } & Tc (K) & $\begin{array}{c}\text { Lattice } \\
\text { Structure }\end{array}$ \\
\hline Zinc (Zn) & 0.85 & HEX \\
\hline Osmium (Os) & 0.66 & HEX \\
\hline Zirconium (Zr) & 0.61 & HEX \\
\hline Americium (Am) & 0.60 & HEX \\
\hline Cadmium (Cd) & 0.517 & HEX \\
\hline Ruthenium (Ru) & 0.49 & HEX \\
\hline Titanium (Ti) & 0.40 & HEX \\
\hline Uranium (U) & 0.20 & ORC \\
\hline Hafnium (Hf) & 0.128 & HEX \\
\hline Iridium (Ir) & 0.1125 & FCC \\
\hline Beryllium (Be) & 0.023 & HEX \\
\hline Tungsten (W) & 0.0154 & BCC \\
\hline Platinum (Pt) & 0.0019 & - \\
\hline Lithium (Li) & 0.0004 & BCC \\
\hline Rhodium (Rh) & 0.000325 & FCC \\
\hline
\end{tabular}

It can be seen that excellent conductors such as copper, silver and gold are not superconductors [166]. This is because they are in a tightly packed FCC lattice structure that creates damping of the electron phonon interaction [166]. The FCC lattice superconductors that appear on the list are able to create adequate lattice vibrations because they have a low modulus of elasticity which promotes phonon-mediated electron coupling [166]. 


\section{Meissner Effect}

In 1933, German physicists Walther Meissner and Robert Ochsenfeld discovered that a superconductor would expel its magnetic field when transitioning to its superconducting state [167]-[171]. This phenomenon that would later be known as the Meissner Effect, means that superconductors are perfect diamagnets in addition to being a perfect conductors [167]-[171]. The superconductor will exclude magnetic fields that would have otherwise flowed through it, by means of current loops to cancel these fields out (screening currents) [167]-[171]. However, this cancelling breaks down when the applied magnetic field crosses a critical value $H_{c}$ [167]. After crossing the critical value, superconductivity will completely cease in type I superconductors [168],[169]. In type II superconductors, after crossing the critical value, there will be a mixed state (also known as a vortex state) where the magnetic flux will increasingly penetrate the material even though there will remain to be no resistance to electric current [170],[171]. There is then a second critical applied field strength where superconductivity will completely cease [170],[171].

\section{Inherent Superconductive Traits}

Brothers Fritz and Heinz London showed in 1935 that the magnetic field decays exponentially from the surface, which would later be known as the London penetration depth [172]. Although it provided an explanation for the Meissner effect as well as resistanceless transport through experimental observations, it did not provide microscopic explanations [173], [174]. These microscopic explanations were given in 1957 by BCS theory [175]. 
In 1956, Leon Cooper described a phenomenon where fermions (such as electrons) can have an arbitrarily small attraction towards each other and lead the pair to have a lower energy than the Fermi energy [174]. This shows that the fermions are paired which became known as Cooper pairing [170], [173]-[176]. This is a unique phenomenon that happens at low temperatures. Normally, electrons would repel each other due to each having a negative charge as stated by Coulomb repulsion [170], [173]-[176]. At low temperatures, the positive ions of the lattice will be attracted to the electrons enough to move toward it and create an area of positive charge density surrounding that area in the lattice [170], [173]-[176]. If the positive charge created is high enough, it will attract other electrons and overcome their repulsion to each other to create a pair [170], [173][176]. After this electron-phonon interaction develops a Cooper pair, the result adds the half spin electrons to create a boson [170], [173]-[176].

In 1957, John Bardeen, Leon Cooper, and John Robert Schrieffer found that the condensate of these Cooper pairs could explain, on a microscopic level, superconductivity [170]. Since Cooper pairs become a boson, they can form a large Bose-Einstein condensate with overlapping pairs [170], [173]-[176]. To break these pairs, they would need to break all the pairs that have condensated as a whole, which creates an oppositional barrier and is a crucial necessity for superconductors [170], [173][176]. This was called BCS theory (an abbreviation of their last names) and they went on to receive the Nobel Prize in Physics in 1972 [176]. 


\section{E. Type 2 Superconductors}

In 1957, superconductors were classified into two types when Alexei Alexeyevich Abrikosov investigated what happen in Ginzburg-Landau theory if $k$ were large instead of small where $k$ is the ratio between superconducting penetration depths and coherence length [177],[178]. Type II superconductors are comprised mostly of metallic compounds and alloys which can yield a higher critical temperatures $T_{c}$ than type I superconductors [177],[178]. The superconducting "perovskites" (metal-oxide ceramics that normally have a ratio of 2 metal atoms to every 3 oxygen atoms) also belong to this type II group [177],[178].

The A15 phases are series of intermetallic compounds with the chemical formula $\mathrm{A}_{3} \mathrm{~B}$ (where $\mathrm{A}$ is a transition metal and $\mathrm{B}$ can be any element) and have a specific structure [179]-[184].

The first time the A15 structure compound was observed was in 1931 when an electrolytically deposited layer of tungsten was examined [179]. Several compounds of the A15 structure were discovered in the following years including the discovery of vanadium silicide $\left(\mathrm{V}_{3} \mathrm{Si}\right)$ which exhibited superconductivity at around $17 \mathrm{~K}$ in 1953 [179]-[184].

In 1954, Niobium-tin $\left(\mathrm{Nb}_{3} \mathrm{Sn}\right)$ was discovered to be a superconductor [185],[186]. In 1961, after several years of investigating, this compound showed that it could superconduct with large currents and strong magnetic fields. This gave it practical applications to be used with high-power magnets and electric machinery [185]-[194]. 
Niobium and titanium (NbTi) alloy is a type II superconductor with a critical temperature of 10 Kelvins and a critical magnetic field of 15 Teslas [195]-[198]. In 1962, at Atomics International, T.G. Berlincourt and R.R.Hake discovered the high critical magnetic field and high critical supercurrent density that it processes which gives it a critical role in the superconducting magnet industry [199]-[214]. It has been used in all particle accelerators so far and for the vast majority of MRI systems [199]-[214]. It is a ductile alloy with mechanical properties which make it easy to fabricate and use [199][214].

The first of the oxide superconductors was created in 1973 by a DuPont researcher named Art Sleight [178]. He found that $\mathrm{BaPbO}_{3}$ and $\mathrm{BaBiO}_{3}$ had a critical temperature of $13 \mathrm{~K}$ and in the late 1970 s, other metal oxides were found to be superconductors as well [178].

The field of superconductivity changed dramatically in 1986, when J. Georg Bednorz and K. Alex Müller produced a material, $\mathrm{La}_{2} \mathrm{CuO}_{4}$, with a critical temperature of $35 \mathrm{~K}$ [215]. This material is different from the previous classes of material in that it has a complex crystal structure made from several components, based around copper oxide units [215]. This discovery earned Bednorz and Müller a Nobel Prize, and was followed a year later by the discovery, by Paul Chu and colleagues, of a superconducting ceramic, $\mathrm{YBa}_{2} \mathrm{Cu}_{3} \mathrm{O}_{7}$ (often abbreviated to $\mathrm{YBCO}$ or 1-2-3 from the ratio of its metal atoms) [215]. This ceramic had an even higher critical temperature of around $92 \mathrm{~K}$ [215]. This meant for the first time that a material exhibited superconducting behavior at temperatures 
above that of liquid nitrogen $(77 \mathrm{~K})$, which is much cheaper and easier to handle than liquid helium [215].

In July 30, 1998, Scientists in Rüschlikon, Switzerland succeeded in doubling the transition temperature at which a material becomes superconducting and loses all resistance to the transport of electrical current [216]. In a paper published in "Nature", a group of scientists from IBM's Zurich Research Laboratory, the Universities of Geneva and Neuchatel (Switzerland), as well as Antwerp (Belgium), reported the successful incorporation of strain into the atomic lattice of a superconducting oxide film, thereby raising the transition temperature of the oxide material from 25 to 49 Kelvin [216]. Besides having practical significance and potential for new record transition temperatures, this finding also highlighted the role played by atomic lattice parameters in the mechanism of superconductivity [216].

The theory of type II superconductor and the discovery of superfluidity in helium3 lead Alexei Alexeyevich Abrikosov, Vitaly Ginzburg, and Anthony James Leggett to be awarded the Nobel Prize in October 7, 2003 [216]. According to their work, vortex lines in a superfluid are analogous to the flux lines that occur in a type II superconductor when it is placed in a magnetic field [216]. In rotating superfluid ${ }^{3} \mathrm{He}$, the vortex structure is particularly rich [216].

The work of Anthony J. Leggett was crucial for understanding the order parameter structure in the superfluid phases of ${ }^{3} \mathrm{He}[216],[217]$. His discovery was that several simultaneously broken symmetries can appear in condensed matter [216],[217]. 
This allowed for a deeper understanding of complex phase transitions in fields such as liquid crystal physics, particle physics and cosmology [217].

In 2015, an article published in Nature by researchers of the Max Planck Institute suggested that under extreme pressure, $\mathrm{H}_{2} \mathrm{~S}$ transitioned to $\mathrm{H}_{3} \mathrm{~S}$ and entered a superconductive state with a transition temperature of $203 \mathrm{~K}$ [218]. The pressure used was 150 gigapascals which is 1.5 million times atmospheric pressure, in a diamond anvil cell [218]. As of 2015, this gives hydrogen sulfide the highest accepted superconducting critical temperature [218]-[228]. By substituting a small part of sulfur with phosphorus and using even higher pressures than what was used for the $203 \mathrm{~K} T_{c}$, it has been predicted that it may be possible to raise the critical temperature to above $0{ }^{\circ} \mathrm{C}$ and even achieve room-temperature superconductivity [218]-[228]. Their research suggests that other hydrogen compounds could superconduct at up to $260 \mathrm{~K}$ which would match up with the original research of Ashcroft [217],[225],[226].

It is important to note that 150 gigapascals is an extremely high pressure to sustain and work with which limits the practicality of using hydrogen sulfide in many applications. Mercury barium calcium copper oxide $\left(\mathrm{H}_{\mathrm{g}} \mathrm{Ba}_{2} \mathrm{Ca}_{2} \mathrm{Cu}_{3} \mathrm{O}_{8}\right)$ remains as the highest transition temperature superconductor at ambient pressure that has been confirmed by multiple independent research groups, with a $T_{c}$ of $133 \mathrm{~K}$ [229].

\section{F. Magnetism}

Examination of the volume of prior work [232]-[272] done to characterize and model the behaviour of magnetic vortices in layered superconductors leads to the conclusion that pancake vortices would be the preferred vortex form if this extremely 
anisotropic material (the ratio of the in-plane resistivity to the normal direction resistivity in pure multilayer graphene is greater than 100,000:1) is superconducting [273]-[274]. A more specific conclusion [267], is that for graphene stacks with interlayer distances of 0.2 $\mathrm{nm}$ and relatively long magnetic penetration depths (graphene and graphite are diamagnetic materials with high levels of magnetic anisotropy [275], [276]) that the interlayer coupling, if the material was superconductive with a magnetic self-pinning attractive force between pancake vortices in layers $i$ and $j$ is given as follows:

$$
F\left(\mathbf{r}_{j}, j, i\right) \approx-\rho\left(\phi_{j}\right)\left(\frac{\varphi_{0}}{2 \pi \Lambda}\right)^{2} \frac{\sqrt{\rho^{2}{ }_{j}}+i-j^{2}\left|s^{2}-i-j\right| s}{\rho_{j}}
$$

where:

$$
\Lambda=2 \lambda_{\|}^{2} / s
$$

$\Lambda$ is the two dimensional (2D) thin-film screening length; $\lambda_{\|}$is the effective penetration depth parallel to the graphene planes and $s$ is the interlayer spacing.

Where the layers $i$ and $j$ are adjacent and the vortices are directly vertically aligned, this reduces to:

$$
\mathbf{F}(\mathbf{z}, 0,1) \approx \hat{z} \frac{\varphi_{0}^{2} s^{2}}{16 \pi^{2} \lambda_{\|}^{4}}
$$


It can be seen that that the magnetic pinning force between two pancake vortices in adjacent layers is proportional to the square of the interlayer spacing and inversely proportional to the fourth power of the magnetic field penetration depth within the layer.

The single pancake vortex pinning energy is given by Clem [268] as:

$$
U_{0} \equiv\left(\phi_{0} / 4 \pi\right)^{2} s / \lambda_{a b}^{2}
$$

where $\lambda_{a b}$ is the in-plane penetration depth.

This gives a self-pinning characteristic temperature for a single pancake vortex in $\mathrm{YBa}_{2} \mathrm{Cu}_{3} \mathrm{O}_{7-\mathrm{X}}$ of $1200 \mathrm{~K}$ and vortex motion begins to be a problem at about $1 / 20$ of that temperature. Expressions (5) and (6) below [268] are given for $\mathrm{YBa}_{2} \mathrm{Cu}_{3} \mathrm{O}_{7-\mathrm{X}}$ :

$$
U_{0} / k_{B}=1200 \mathrm{~K}
$$

where the flux motion temperature regime is:

$$
\left(U_{0} / k_{B}\right) / 20=60 \mathrm{~K}
$$

The inter-plane separation of graphene is $0.2 \mathrm{~nm}$ and that of $\mathrm{YBa}_{2} \mathrm{Cu}_{3} \mathrm{O}_{7-\mathrm{X}}$ is $1.2 \mathrm{~nm}$ so if the in-plane penetration depth $\lambda_{a b}$ is the same the expected temperature where flux motion regime begins to be important for graphene would be about $10 \mathrm{~K}$. This is an upper estimate given that the measured conduction anisotropy in graphite is significantly greater than that of $\mathrm{YBa}_{2} \mathrm{Cu}_{3} \mathrm{O}_{7-\mathrm{X}}$. It is expected that realistic estimates for the onset temperature for flux-flow would be much lower. Therefore, the expectation is that, even 
if the material is superconducting with Cooper pairs, the resistance, due to flux flow effects, would be non-zero even at low temperatures and currents.

The strength of attraction of a pancake vortex to a vacancy due to implantation damage is related to the size of the vacancy and the in-plane penetration depth that governs the physical size of the pancake vortex. The pinning force at low temperatures will not be significant in this highly anisotropic material unless pancake vortices, which can be pinned to a vacancy, have numerous similar vortices pinned at vacancies in adjacent layers. Pancake vortex "stacks" can form at these adjacent vacancies, which may result from implantation damage. The pinning force of these stacks would be from the summation of the vortices in the stack's mutual magnetic and Josephson interactions [266].

The pinning energy and the temperature required to "melt" one of these vortex stacks are proportional. Once a stack melts, all of the vortices in the stack are free to move and immediately contribute to resistive losses in the material. This would lead to the expectation of upwards steps in resistance at the melting temperatures of the various height pancake vortex stacks. Pancake vortex stacks will still form, regardless of the presence of implantation damage; however, the probability of adjacent vacancies leading to the formation of vertical stacks would be less in materials without columnar defects to stack along. A doped-while-grown material would therefore be less likely to have sharp steps as the pancake vortices would be less vertically aligned and more tilted away from the normal to the layers (greater spread in layer-to-layer offsets).

Magnetization measurements on thin film superconductors where the penetration depth is many times greater than the film thickness have been done by Berdiyorov [277] 
and a number of theoretical works [275]-[310] that are consistent with the experimentally observed results exist. The results from Berdiyorov are best summarized thus: first, the magnetization is negative and, second, the magnetization has a "valley" or quasiparabolic shape as a function of temperature and/or applied field. This is quite different from what is seen for thicker, classical, samples where pancake vortices are not formed and the applied magnetic field is expelled from the bulk of the sample.

The primary differences that thin films (where the thickness is far less than the magnetic penetration depth) show in comparison to materials with a unity ratio of thickness to penetration depth (in an AC susceptometer) are: (1) a smaller net signal as the screening is smaller and (2) a broader, depressed, transition as a function of temperature due to the field penetration and vortices [311]-[351].

In Hall effect measurements on mixed state layered superconductors with weak flux pinning, it has been widely observed that the presence of a vortex state with mobile vortices often leads to a sign reversal in the Hall voltage as the sample goes through the transition [352]-[355].

\section{G. Resistance versus Temperature}

The Resistance versus Temperature measurement is one of the most basic ways to determine the presence of superconductive properties [415]. Once the critical temperature is passed and the sample goes into the superconducting phase, there will be a sudden drop in resistance [42]-[52], [416].

Prior work on doped HOPG [72] showed that the sample's resistance never went to zero, even at low temperatures. Flux-flow resistance was identified to be the potential reason for non-zero resistance at low temperatures [230], [231]. Flux pinning has been 
indicated as the key to reducing this flux-flow resistance in layered high critical temperature semiconductors [230]-[270]. Consideration must also be given to the nanometer scale thickness of the exfoliated graphene film under test, as it relates to the magnitude of the test current used in $R$ vs. $T$ measurements [412]-[414], [417]-[419]. This current must be as small as possible or it could potentially influence the results due to either local magnetic fields or heating.

Due to the sensitivity of carrying out $R$ vs. $T$ measurements on thin films, numerous data points are taken in order for an accurate result to be attained. In order to lessen the difficulty, greater focus should be placed on the ability to detect when even a small portion of the sample is superconducting, which will lead to much smaller steps than seen in classical superconductors.

The four probe measurements are the industry standard for measuring superconductors as well as semiconductors [86]-[88]. For superconductors, the collinear probe arrangement is more popular due to its stronger signal strength at lower resistances, as well as a lower need for the Hall Effect measurement (as with semiconductors) [86]. This helps to increase the signal to noise ratio as well as eliminating the effect of probe resistance or contact resistance [86], [87].

Two outer probes are used to source current while two other probes placed between them measure the voltage drop [86]-[88]. 


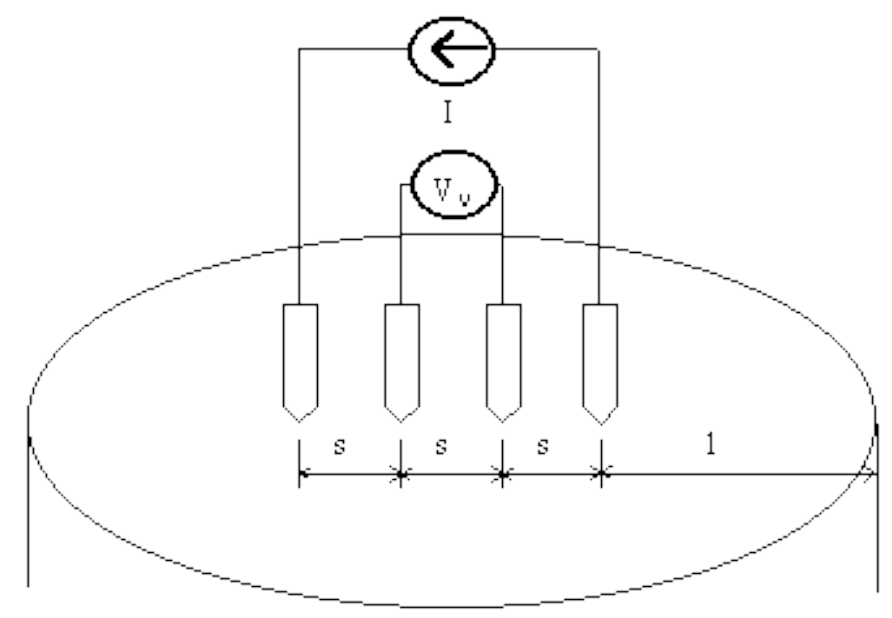

Figure 2: Four probe measurement. Collinear setup. Picture courtesy of [89].

The resistivity is then calculated by using the following:

$$
\rho_{0}=2 a \pi s \frac{V}{I}=a \rho_{0}
$$

Where ' $\mathbf{a}$ ' is the thickness correction factor and $\mathbf{s}$ is the spacing between probes, presumed to be equal [89]. Since graphene is extremely thin, the correction factor will be determined by use of the plot below, and substitute it for $\mathbf{K}$ in the following formula, where $\mathbf{t}$ is the thickness and $\mathbf{m}$ is the slope:

$$
a=K\left(\frac{t}{s}\right)^{m}
$$




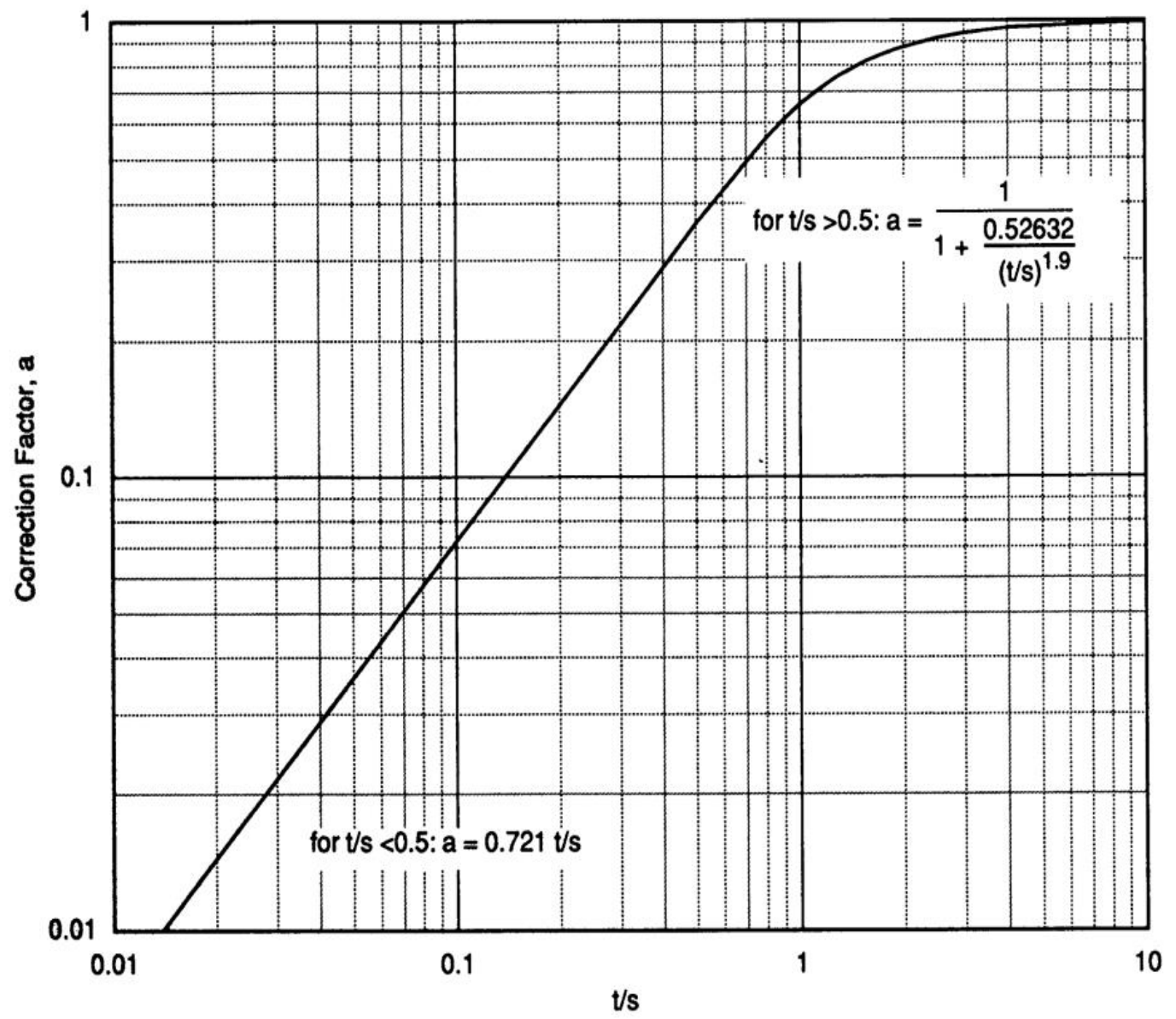

Figure 3: Correction factor plot. Picture courtesy of [89].

The delta mode method being implemented by the Keithley Delta Mode System 6221/2182A is displayed below: 


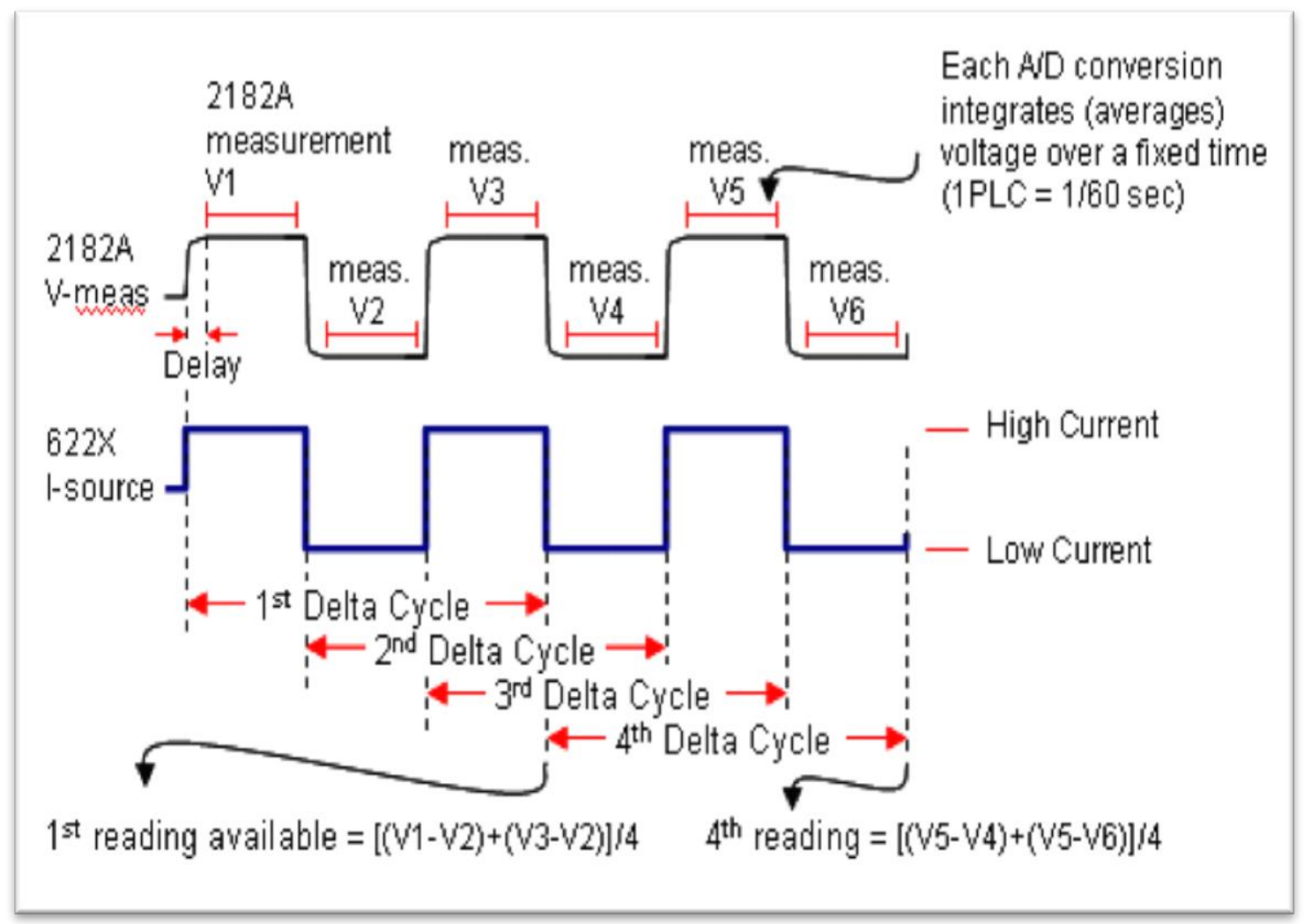

Figure 4: Delta Mode reading calculation. Screenshot of Keithley's help menu in test program.

Since the current being supplied is switching polarity but remains at the same absolute value, the difference in potential between V1 and V2 is twice as large as the voltage should be. The same can be said for the difference between V3 and V2. Therefore, we arrive at the expression:

$$
\begin{gathered}
V_{a}=\text { negative_edge }=\left(V_{1}-V_{2}\right) / 2 \\
V_{b}=\text { positive_edge }=\left(V_{3}-V_{2}\right) / 2
\end{gathered}
$$

The final voltage reading would be the average of these two readings: 


$$
\begin{gathered}
V_{f}=\left(V_{s}+V_{b}\right) / 2 \\
V_{f}=\left[\left(V_{1}-V_{2}\right) / 2+\left(V_{3}-V_{2}\right) / 2\right] / 2 \\
V_{f}=\left[\left(V_{1}-V_{2}\right) / 2+\left(V_{3}-V_{2}\right)\right] / 4
\end{gathered}
$$

This method is beneficial as it eliminates linear thermoelectric drift. The drift only has to be linear with respect to the 3 points being measured, as seen in the figure below.

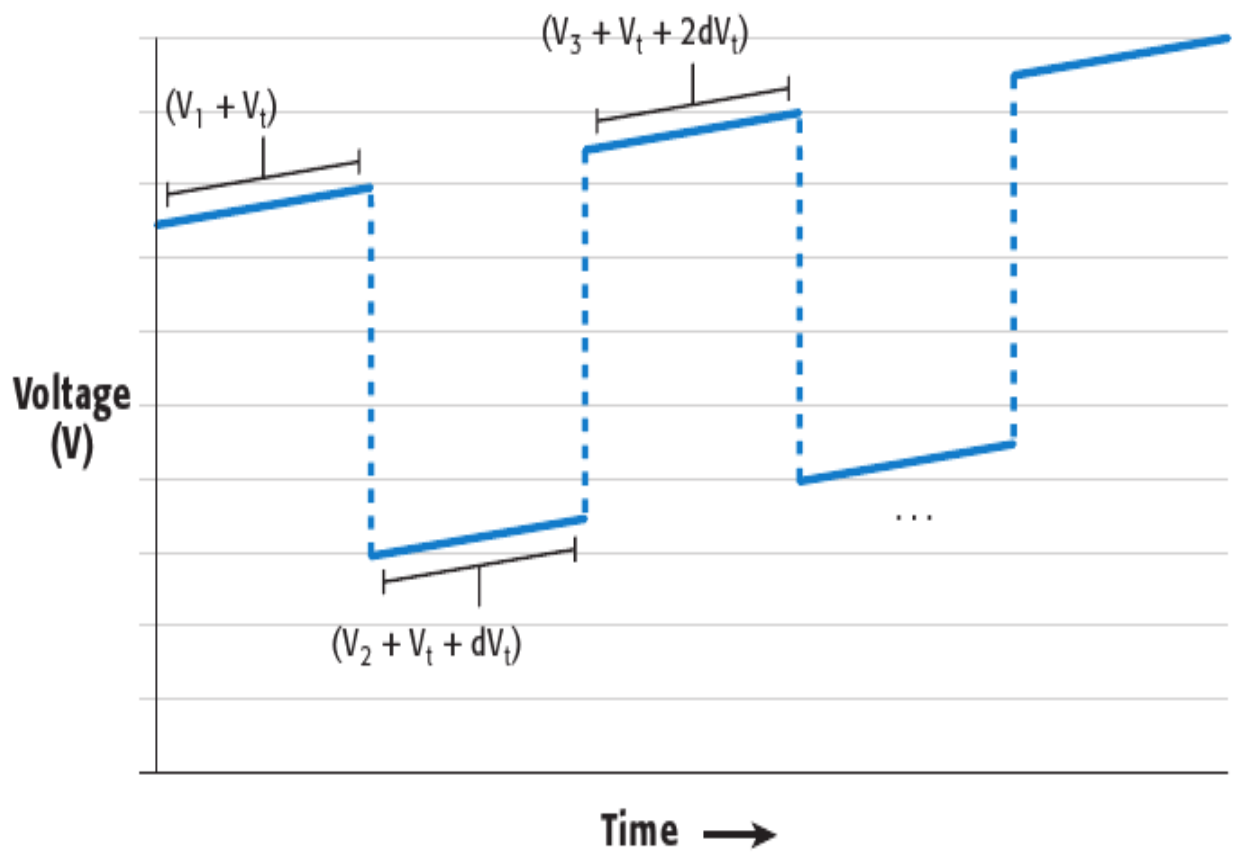

Figure 5: Delta Mode Cancelling Thermoelectric drift.

Letting $V_{t}$ represent the offset caused by the thermoelectric drift and $d_{t}$ represent the change in the offset of thermoelectric drift since the initial $V_{t}$ (to simulate drift going upwards or downwards):

$$
\begin{gathered}
V_{a}=\left[\left(V_{1}+V_{t}\right)-\left(V_{2}+V_{t}+d V_{t}\right)\right] / 2 \\
V_{a}=\left(V_{1}-V_{2}-d V_{t}\right) / 2
\end{gathered}
$$




$$
\begin{gathered}
V_{b}=\left[\left(V_{3}+V_{t}+2 d V_{t}\right)-\left(V_{2}+V_{t}+d V_{t}\right)\right] / 2 \\
V_{b}=\left(V_{3}+2 d V_{t}-V_{2}-d V_{t}\right) / 2
\end{gathered}
$$

$V_{t}$ is removed in expanding $V_{a}$ and $V_{b}$. Once $V_{f}$ is calculated, we see that all the $\mathrm{dV}_{t} s$ are cancelled:

$$
\begin{gathered}
V_{f}=\left(V_{a}+V_{b}\right) / 2 \\
V_{f}=\left[\left(V_{1}-V_{2}+d V_{t}\right) / 2+\left(V_{3}+2 d V_{t}-V_{2}-d V_{t}\right) / 2\right] / 2 \\
V_{f}=\left(V_{1}-V_{2}-d V_{t}+V_{3}+2 d V_{t}-V_{2}-d V_{t}\right) / 4 \\
V_{f}=\left[\left(V_{1}-V_{2}\right)+\left(V_{3}-V_{2}\right)\right] / 4
\end{gathered}
$$

This final value for $\mathrm{V}_{\mathrm{f}}$ represents a single reading that has removed any linear thermoelectric drift that occurred between $\mathrm{V}_{1}$ and $\mathrm{V}_{3}$.

\section{H. Magnetic Field Measurement}

To detect the Meissner Effect, a susceptometer (also called a magnetometer) is used to detect the shift in magnetism. 


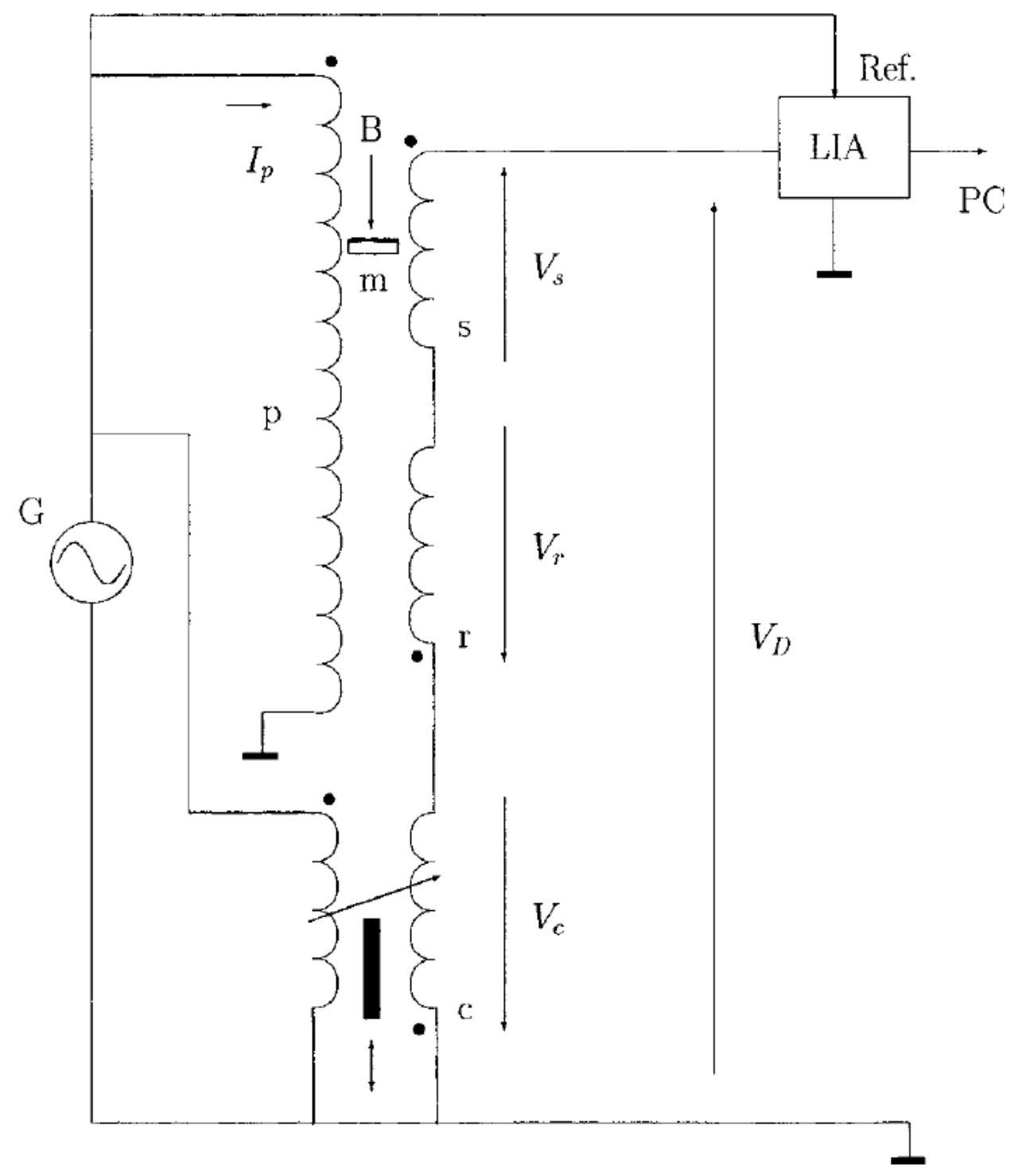

Figure 6: Schematic of magnetometer. Picture courtesy of [90].

$\mathbf{M}$ represents the material, $\mathbf{s}$ is the coil that is around the material, $\mathbf{r}$ is the reference coil, $\boldsymbol{c}$ is the compensation for compensation of imbalance between $V_{s}$ and $V_{r}$ with no sample present. $\mathbf{p}$ is outer coil generating the magnetic field and $\mathbf{G}$ is the current going to the external coil [90]. 
When $V_{r}$ and $V_{s}$ are balanced, (no magnetic field change) $V_{d}$ should be zero, otherwise the material is causing an imbalance, repelling the magnetic field. To quench the superconducting properties, a strong magnetic field is applied to the sample [387][411].

\section{EXPERIMENTAL}

All samples used in this work were HOPG ZYH specimens with a mosaic spread of $3.5^{\circ} \pm 1.5^{\circ}$, grain size of $30-40 \mathrm{~nm}$ and density of $2.255-2.265 \mathrm{~g} / \mathrm{cm}^{3}$. Prior to the first use, 10 to 15 monatomic layers were removed from every sample by exfoliation to ensure a pristine layer for initial testing. More than 20 bulk HOPG samples and over 35 exfoliated thin graphite layers, - both doped and non-doped, - were tested in this work. For all samples, preparation proceeded as follows:

All samples were scratched on one side, to indicate the bottom of the sample. Although the HOPG samples have two working sides, it was important to keep track of which side was the bottom after the single sided cleaning and doping process. Before doping, regardless of whether doping was by implantation or through Chemical Vapor Deposition growth, the sample was placed into a closed cycle refrigeration system and the Resistance vs. Temperature ( $R$ vs. $T$ ) characteristic of the HOPG was measured.

\section{A. Exoliation}

Exfoliation, pioneered by Novoselov and Geim [92]-[108], has been used to obtain samples for measurements by a large number of other researchers and is widely utilized.

Kapton $^{\circledR}$ tape made of polymide [85] has in the past been used successfully to peel a layer of graphene from highly oriented pyrolytic graphite. This tape is meant for high 
vacuum usage due to its uniform silicone adhesive layer which ensures air bubbles will not be trapped in between the tape and the object it is adhering to [85]. Air bubbles will lead to outgassing that can make the vacuum pressure unstable, which is undesirable.

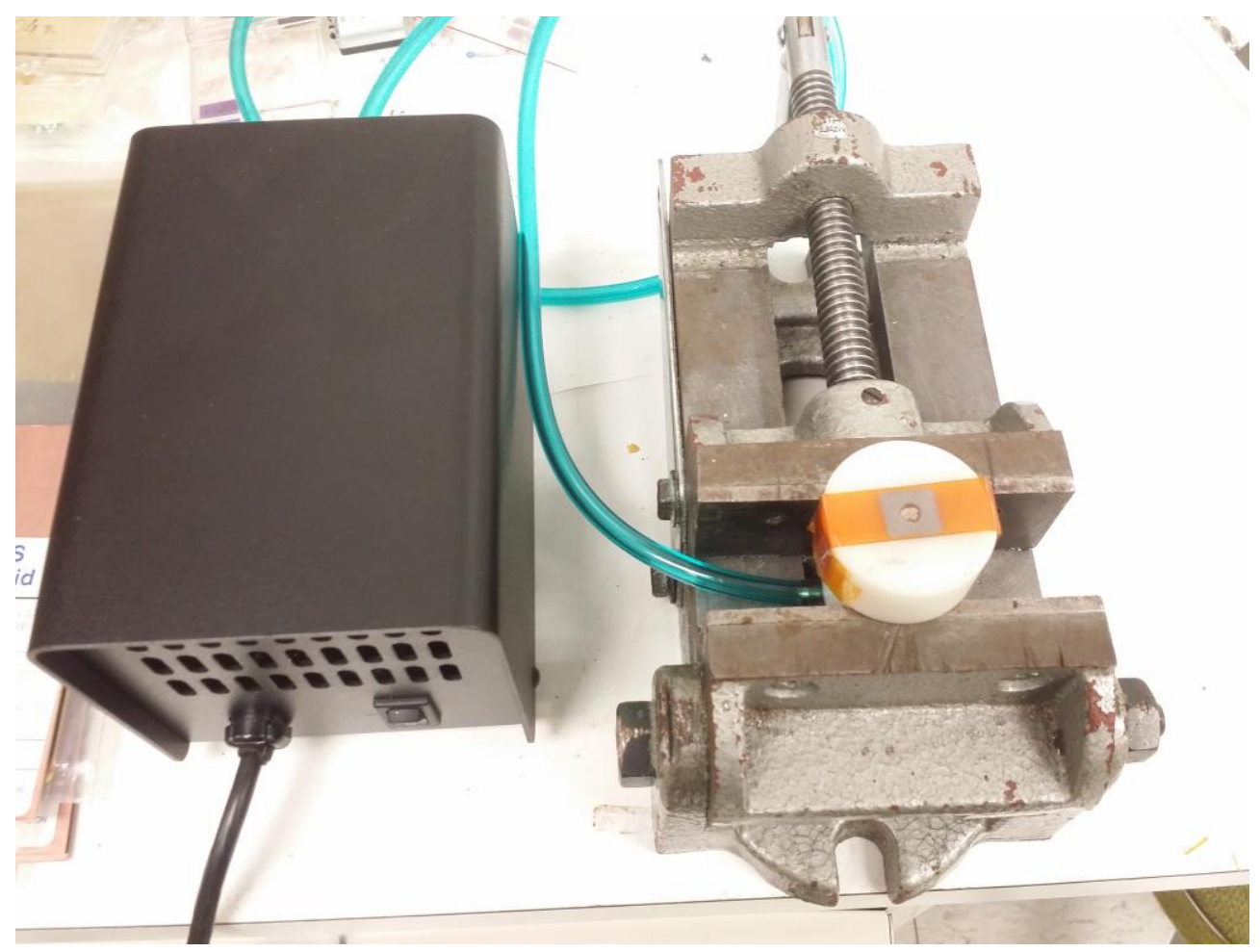

Figure 7: System created for sample preparation. Chuck with through hole to create vacuum, capable of holding down HOPG sample. Silicon Kapton ${ }^{\circledR}$ tape was added to give a soft surface for the HOPG to rest, which was found to give a better vacuum seal. Vacuum system with tubes connected to chuck shown on the left. 


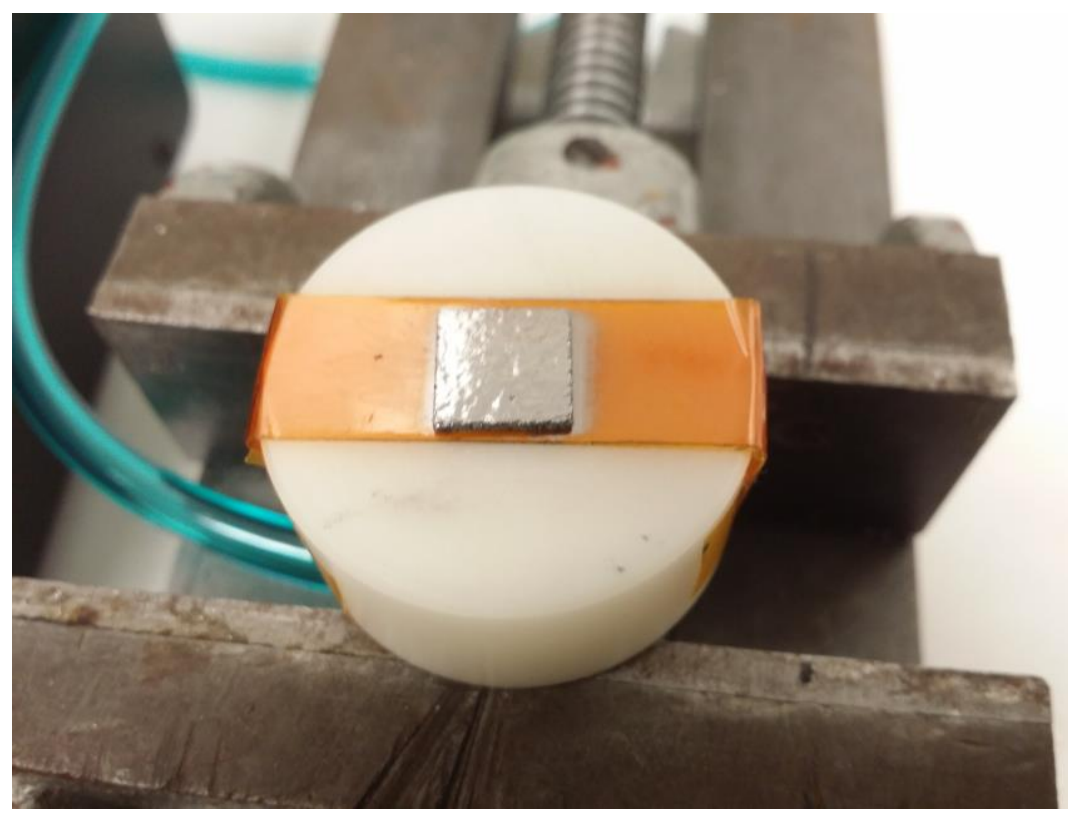

Figure 8: Sample preparation system with HOPG sample secured to chuck via vacuum seal. A piece of Kapton ${ }^{\circledR}$ tape will be laid on top of the HOPG, and carefully pressed down to remove all air bubbles.

This makes Kapton tape ideal for the application of peeling graphene since an air bubble would translate into an area of graphene that may not lift off, leading to a noncontinuous flake of graphene. Also, the resistance of the Kapton ${ }^{\circledR}$ tape, in this application, approaches infinity as its resistance is much higher than the resistant measurement devices can read (thus, not contributing to the resistance of the sample being measured).

Theoretically, this process should only remove a single layer of graphite (graphene). Practically however, this is not generally the case. There are many factors causing several layers to be peeled off simultaneously. Examples such as: an uneven sample surface allowing the lowest layers to pull top layers along with it and layers simply being stuck together with a force greater on the top layer than on the bottom. In order to mitigate this 
effect, a second or even third peel of the initial peel can be taken (thus, dividing the layers of graphene to eventually approach 1).

The move from bulk ion-implanted HOPG to exfoliated (peeled) graphene from ionimplanted HOPG allowed the removal of bulk effects from the physics occurring in the first $20 \mathrm{~nm}$ where the greatest damage from the dopant implantation were located [56]. The use of phosphine as a dopant in doped-while-grown plasma Chemical Vapor Deposition films was observed to minimize the implantation damage.

Removal of graphene from tape was investigated using solvents that would dissolve the adhesive. Having graphene without tape would allow for uniform thermal expansion as well as being able to test the doped side directly. It would also allow for closer stacking of samples during susceptibility measurements, which would give a stronger response since the effective sample thickness could be made to be much larger than the London penetration depth.

Each solvent was placed in a glass beaker and graphene on either Kapton ${ }^{\circledR}$ or $\operatorname{Scotch}^{\circledR}$ general purpose tape was placed in the beaker, completely submerged by the solvent. The beaker was then covered with plastic wrap to avoid evaporation and placed in a fume hood for over 24 hours. After that time, the graphene was removed, placed on a lint free towel and removal from the tape was attempted using two tweezers. 
Table 3. Solvents tested for graphene lift off from Kapton ${ }^{\circledR}$ vacuum tape and general purpose $\operatorname{Scotch}^{\circledR}$ tape. The green shade denotes lift off while still floating in the solvent. Yellow shading denotes lift off with the aid of tweezers. Red denotes no lift off.

\begin{tabular}{|c|c|c|c|c|c|c|}
\hline Solvent & Tape & $\begin{array}{l}\text { Lift } \\
\text { Off? } \\
(\mathbf{y} / \mathbf{n})\end{array}$ & Difficulty & $\begin{array}{c}\text { Time } \\
\text { In } \\
\text { solvent }\end{array}$ & Comments & Solubility \\
\hline $\begin{array}{l}3 \mathrm{M}^{\circledR} \text { General } \\
\text { Purpose } \\
\text { Adhesive } \\
\text { Cleaner }\end{array}$ & Vacuum & Yes & Hard & $24 \mathrm{hrs}$ & Difficult to get full piece to remove. & \\
\hline $\begin{array}{l}3 \mathrm{M}^{\circledR} \text { General } \\
\text { Purpose } \\
\text { Adhesive } \\
\text { Cleaner }\end{array}$ & $\operatorname{Scotch}^{\circledR}$ & Yes & Hard & $24 \mathrm{hrs}$ & Difficult to get full piece to remove. & \\
\hline TCE & Vacuum & Yes & Easy & $24 \mathrm{hrs}$ & Full lift off in liquid. & $\begin{array}{l}\text { ether, ethanol, } \\
\text { chloroform }\end{array}$ \\
\hline TCE & $\operatorname{Scotch}^{\circledast}$ & No & Very Hard & $24 \mathrm{hrs}$ & $\begin{array}{l}\text { Scotch tape curled and could not remove } \\
\text { graphene }\end{array}$ & \\
\hline Kerosene & Vacuum & Yes & Hard & $24 \mathrm{hrs}$ & Partial Lift off. Some flakes still remain. & \\
\hline Kerosene & $\operatorname{Scotch}^{\circledast}$ & No & Very Hard & $24 \mathrm{hrs}$ & & \\
\hline Goof-Off $^{\circledR}$ & Vacuum & Yes & Easy & $24 \mathrm{hrs}$ & $\begin{array}{l}\text { Graphene slid off tape when pushed } \\
\text { horizontally }\end{array}$ & \\
\hline Mineral Spirits & Vacuum & Yes & Easy & $27 \mathrm{hrs}$ & $\begin{array}{l}\text { Graphene stuck to napkin when put } \\
\text { upside-down and only used tweezers to } \\
\text { lift tape off. }\end{array}$ & \\
\hline Mineral Spirits & $\operatorname{Scotch}^{\circledR}$ & No & Very Hard & $27 \mathrm{hrs}$ & & \\
\hline Xylene & Vacuum & Yes & Easy & $27 \mathrm{hrs}$ & $\begin{array}{l}\text { Extremely easy to remove. Graphene } \\
\text { stuck to napkin when put upside-down } \\
\text { and only used tweezers to lift tape off. }\end{array}$ & $\begin{array}{l}\text { non-polar } \\
\text { solvents such } \\
\text { as aromatic } \\
\text { hydrocarbons }\end{array}$ \\
\hline Xylene & $\operatorname{Scotch}^{\circledR}$ & Yes & Easy & $27 \mathrm{hrs}$ & $\begin{array}{l}\text { Extremely easy to remove. Graphene } \\
\text { stuck to napkin when put upside-down } \\
\text { and only used tweezers to lift tape off. } \\
\text { Easiest scotch tape removal. }\end{array}$ & \\
\hline MEK & Vacuum & Yes & Easy & $27 \mathrm{hrs}$ & As easy as Xylene, Mineral Spirits. & Water \\
\hline MEK & $\operatorname{Scotch}^{\circledR}$ & No & Very Hard & $27 \mathrm{hrs}$ & $\begin{array}{l}\text { Pieces came off but scotch tape was } \\
\text { gooey and staying on. }\end{array}$ & \\
\hline Goo-Gone $^{\circledR}$ & Vacuum & Yes & Easy & $27 \mathrm{hrs}$ & & \\
\hline Goo-Gone $^{\circledR}$ & $\operatorname{Scotch}^{\circledR}$ & Yes & Medium & $27 \mathrm{hrs}$ & $\begin{array}{l}\text { Partially stuck to scotch tape but able to } \\
\text { get it off in somewhat big flakes. }\end{array}$ & \\
\hline Krud-Kutter ${ }^{(B)}$ & Vacuum & Yes & Easy & $27 \mathrm{hrs}$ & & \\
\hline Krud-Kutter $^{\circledR}$ & $\operatorname{Scotch}^{\circledR}$ & Yes & Medium & $27 \mathrm{hrs}$ & Very similar to goo-gone. & \\
\hline Turpentine & Vacuum & Yes & Easy & $27 \mathrm{hrs}$ & & \\
\hline Turpentine & $\operatorname{Scotch}^{\circledR}$ & Yes & Medium & $27 \mathrm{hrs}$ & & \\
\hline
\end{tabular}


As seen in Table 3, TCE, Xylene and MEK were the best solvents from the ones we tested. These either floated off while still in the solvent or were taken off with little to no resistance. Out of those 3 , MEK has the added benefit of being soluble in water, making the post removal or residue safe and easy.

For fear of altering the sample, the tape removal process was implemented in later testing. However, this may be an important finding for future work.

\section{B. Resistance versus Temperature Measurement}

In order to achieve a high level of sensitivity, there should be little to no noise, little to no thermoelectric error and high precision present in the measuring equipment. The Keithley Delta Mode System 6221/2182A in conjunction with LabView allows for the noise to be minimized, thermoelectric error to be eliminated completely while being able to take measurements at a $1 \mathrm{nV}$ resolution [86].

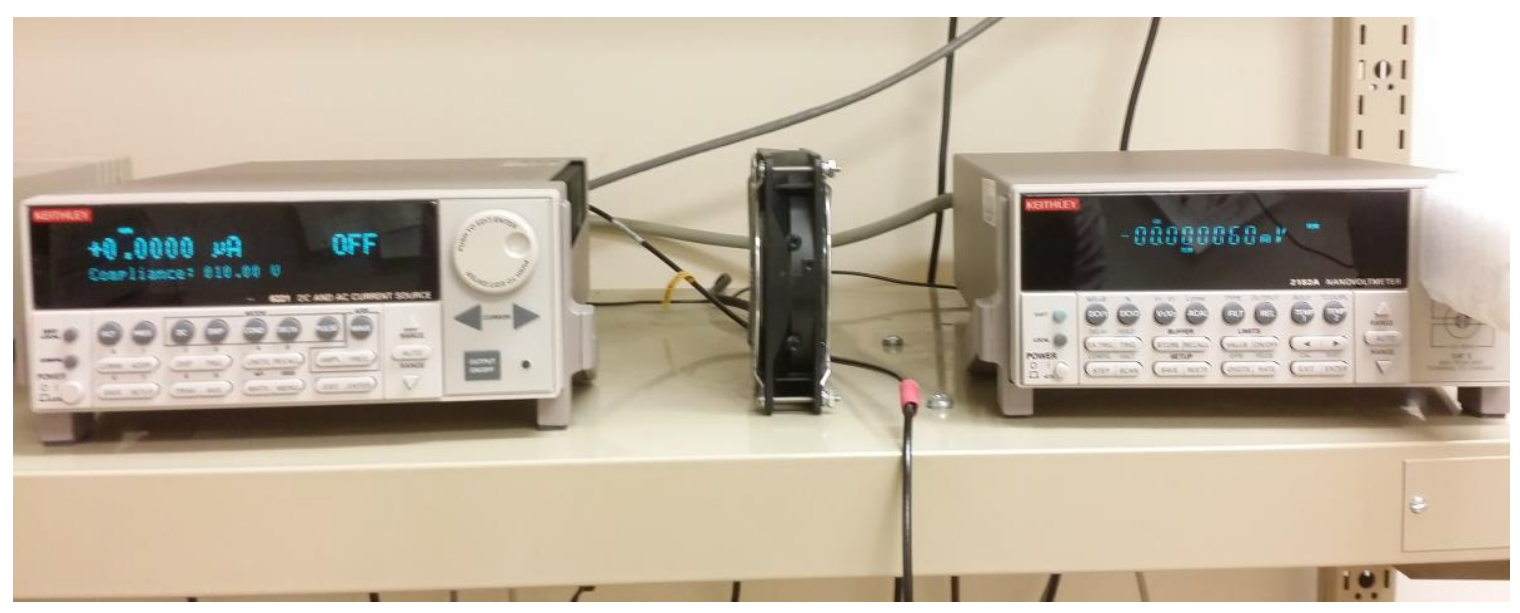

Figure 9: Keithley 6221 DC and AC current source (on left) and Keithley 2182A nanovoltmeter (on right) with external cooling fan in between to keep airflow constant for cooling of the device. 


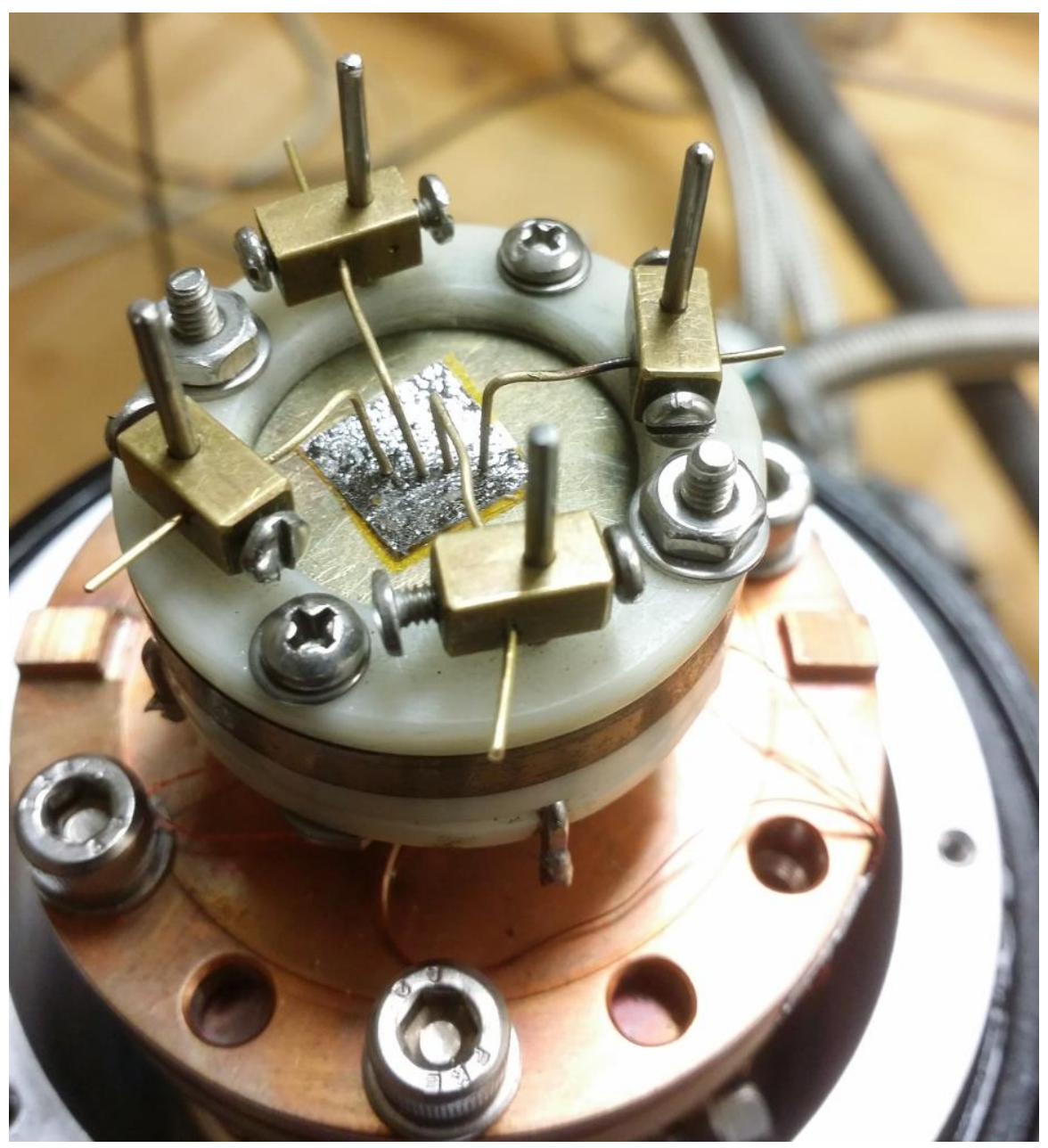

Figure 10: The peeled sample lifted from the HOPG, loaded into the cryogenic system where probes are placed collinearly onto a continuous area of graphene.

The probes are placed firmly enough to maintain contact, but not so firm that they puncture the material. This is verified after the sample is taken out of the chamber by making sure no holes have been created.

During this measurement portion, the temperature is brought down to 2.5 Kelvin by a compressed helium cooled cryogenic system. This is done under vacuum for the health of the cryogenic system but should not affect the measurements. 


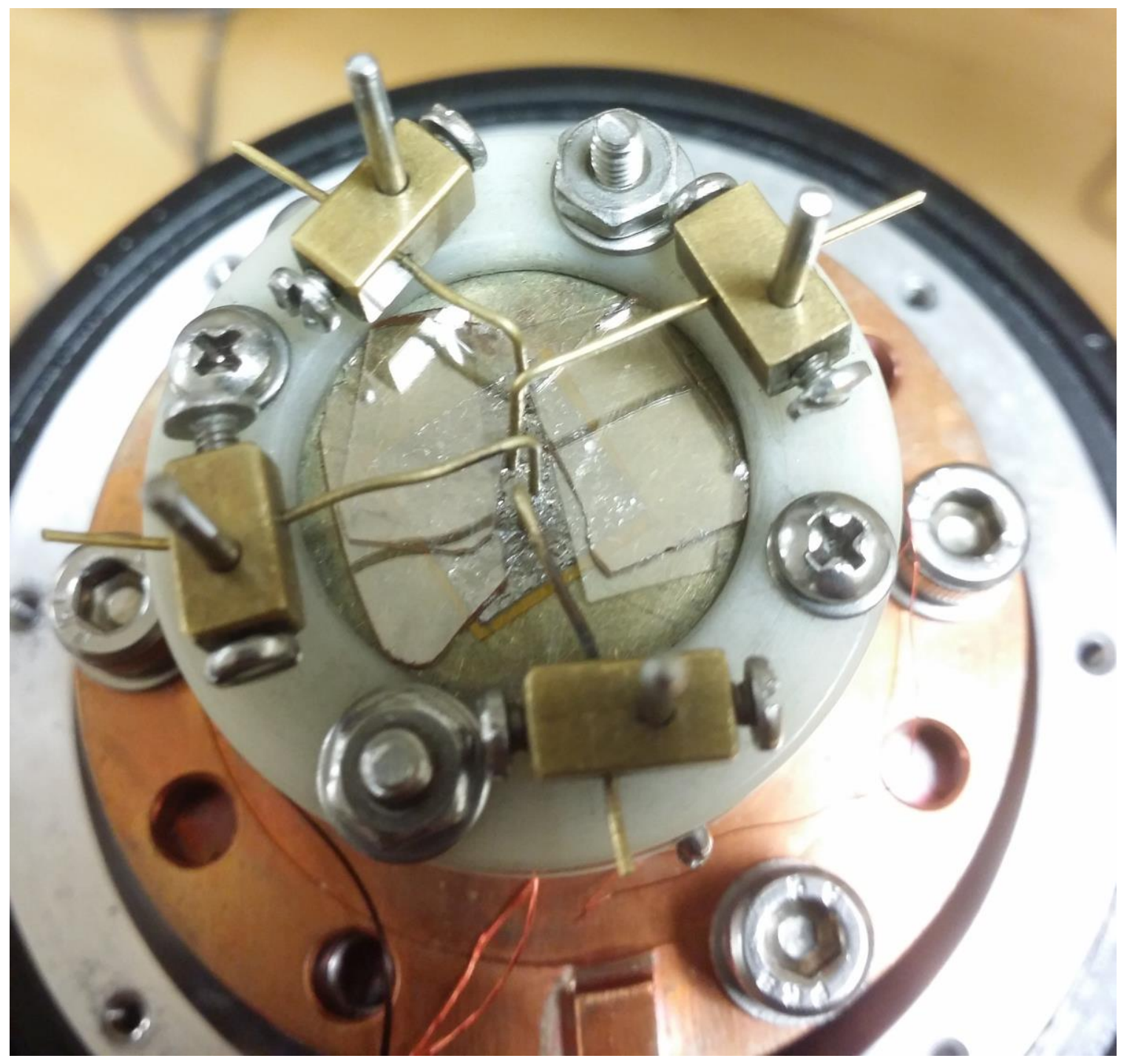

Figure 11: Colinear Probes positioned on sample with broken pieces of Lanthanum Aluminate (LaAl) placed near the edges to prevent curling.

After several trial runs of bringing samples down to 2.5 Kelvin and back to room temperature, the ends of the samples were observed to be curling upwards. This curling had a noticeable change on the response of the sample, thus a method to secure the 
sample was achieved by placing Lanthanum Aluminate ( $\mathrm{LaAl})$ on the edges. LaAl was chosen for its high thermal conductivity, which would ensure that the sample temperature itself would not be affected. LaAl was also chosen due to its low electrical conductivity, which ensured that there would be no shorting or interference between collinear probes.

When signs of superconductivity are present, additional $R$ vs. $T$ measurements is carried out in the presence of a magnetic field. Characteristics that are reduced substantially in the presence of a magnetic field can be interpreted as possibly being due to superconducting properties so it is also important to apply this magnetic field without physically disturbing the sample. 


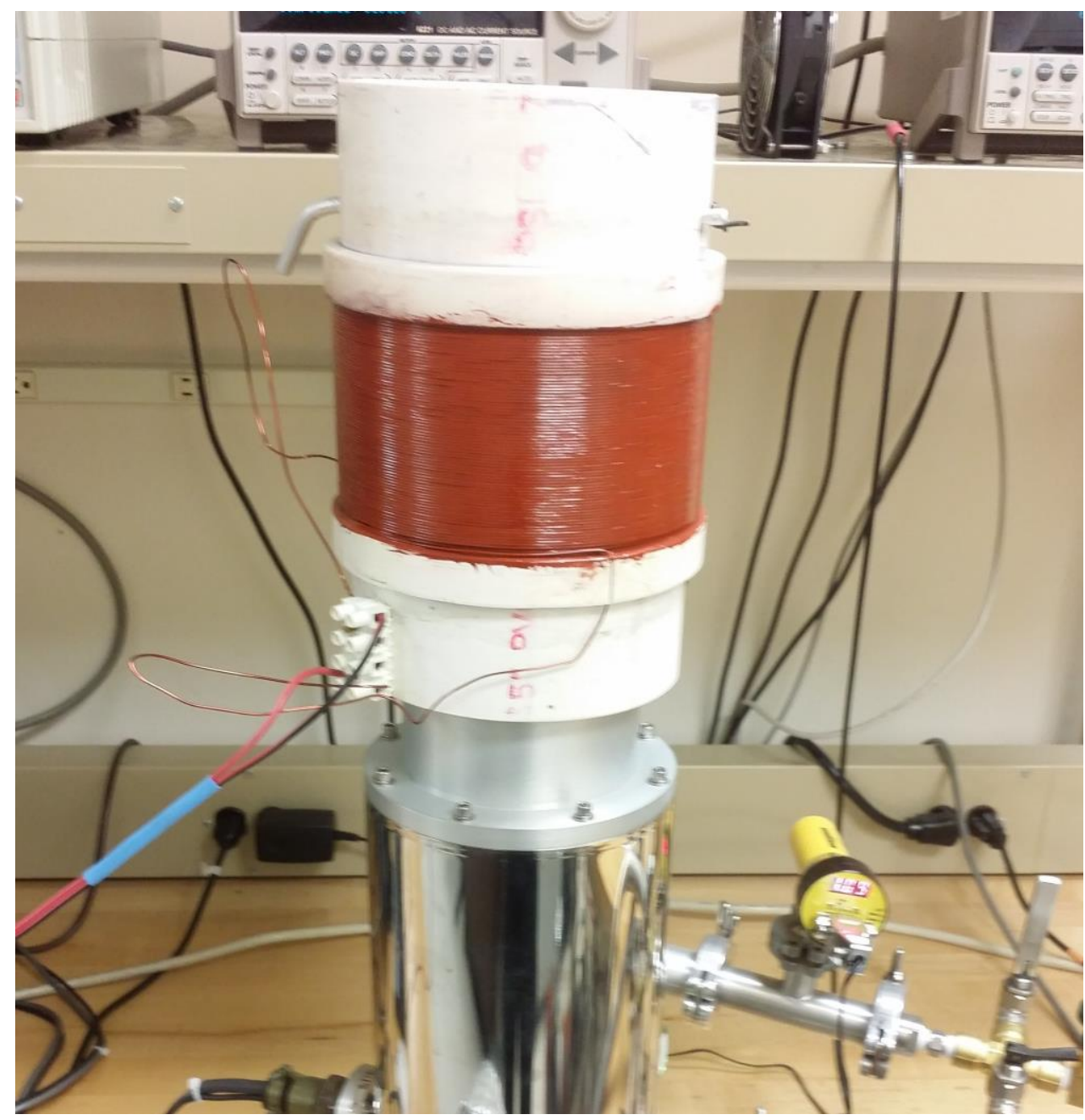

Figure 12: Coil created in-house to generate magnetic field. This slides over the outer shield and was adjusted to make the center of the coils on the same plane as the sample. The ends of the copper coils are connected to a 4 amp DC source.

\section{Programming for Resistance versus Temperature Measurements}

Extensive programming was done in LabView to efficiently carry out the laborious runs. This was an ongoing process refining and optimization. Additions were made to prevent any previous errors that we saw from happening again. One of the first programs that were created for the $R$ vs. $T$ measurements was the data acquisition VI. This fully automated the Delta measurements as well as monitoring the temperature in the 
cryogenic system. The program takes an initial temperature measurement to determine if the run is cooling or warming and then determines if it will need to turn the heaters on later. If it determines that it is cooling, it will take a packet of 250 measurements and then divide them into 10 sub packets. Each of those 10 sub packets will be an average of 25 measurements. For warming, it will take the full packet of 250 measurements and simply get the average to give 1 average measurement from the entire packet. It then displays this information and records it along with other pertinent information, to a Comma Separated Value file before moving on to the next measurement. Comma Separate Value files allow the data to be saved in a table structure while being able to opened by text editors or as spreadsheets. For cooling runs, the program text messages the user to alert them that the cooling run has completed. This lets the user know that they need to turn off the compressor as well as the water that cools the compressor. Then it sends the Comma Separate Value file and the picture of the $R$ vs $T$ on screen data display to everyone on the team which it also does at the end of the warming run (see Appendix 26 to Appendix 51 ) .

Later, this program was turned to a subVI with an outer VI that controlled the compressor (see Appendix 25 to Appendix 38 ). 


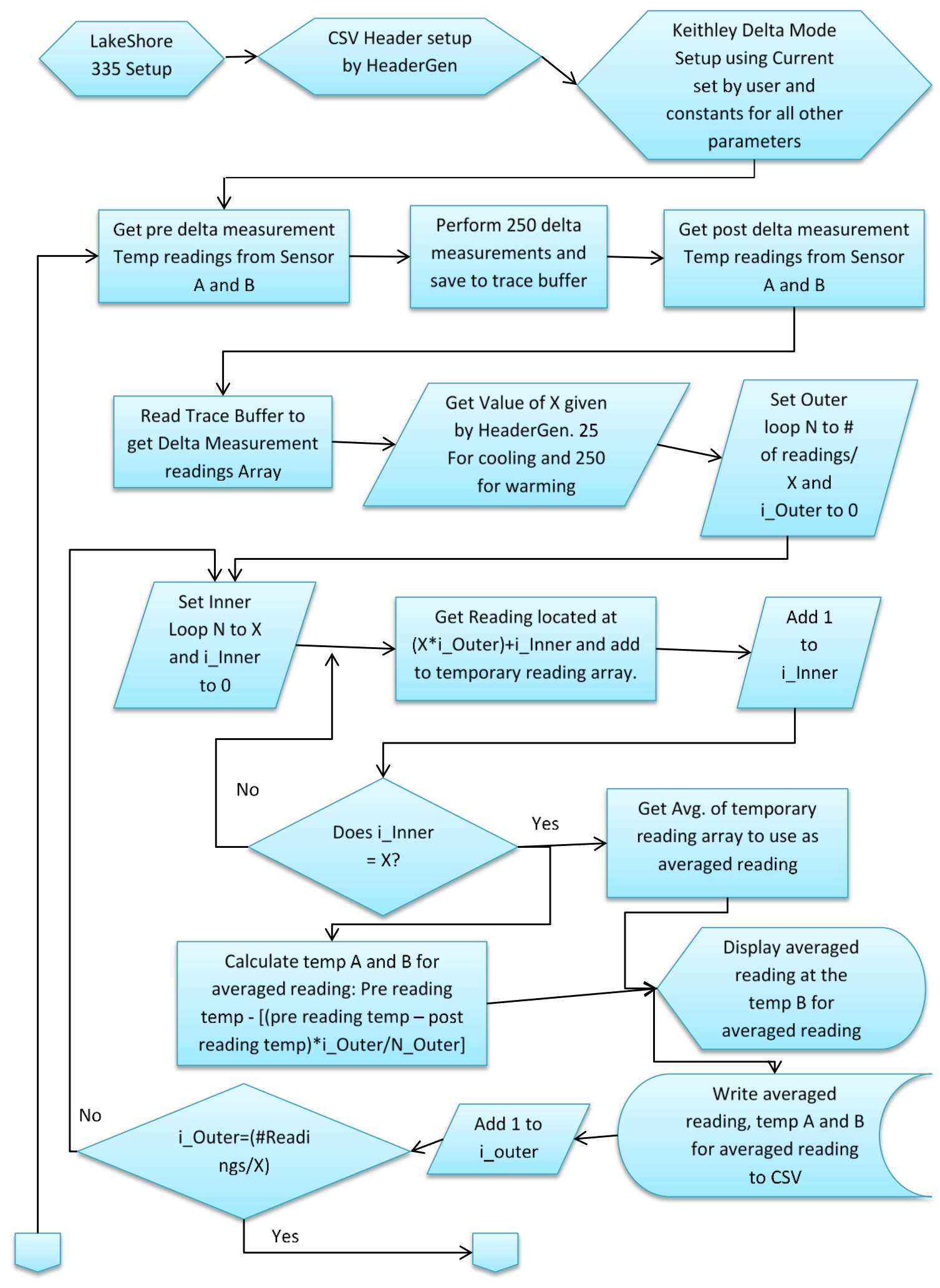

Figure 13: Flow chart for $R$ vs. $T$ measurement subVI for data acquisition. 


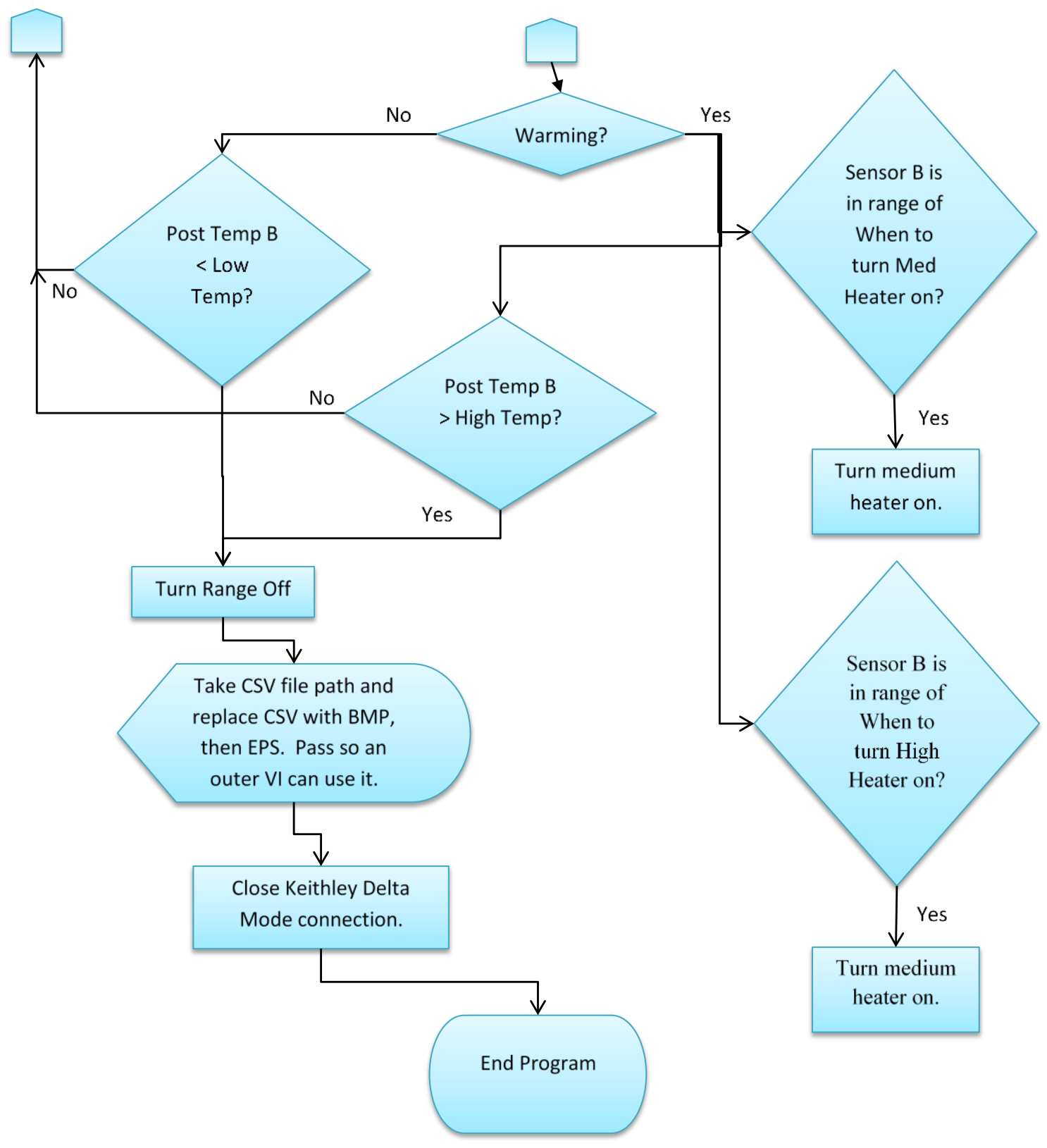

Figure 14: Flow chart for $R$ vs. $T$ measurement subVI for data acquisition continued. 
All measurements were averaged delta mode in nature and each data point recorded was the result of 250 individual readings averaged together. Measurement speed was approximately 10 seconds per final averaged data point recorded. All measurements were made at the minimum practical stimulus current, always $10 \mu \mathrm{A}$ or less (typically $1 \mu \mathrm{A}$ ), to avoid current induced degradation of the responses. After initial testing, the samples were doped with phosphorous using either ion-implantation or by Chemical Vapor Deposition growth with phosphine in the gas mixture.

\section{Doping $H O P G$}

One electron donor (phosphorous), and an electron acceptor (boron) were selected initially for testing the hypothesis. The primary doping method of ion implantation was preferred simply due to expediency. Low energies and doses were used to minimize damage.

A response suggestive of superconductivity in phosphorous (electron donor) implanted HOPG was observed [72]. The estimated critical temperature in this system is in excess of $100 \mathrm{~K}$ and, may very likely be considerably higher if damage incurred during the doping could be further minimized. It must be stated that the observed "superconductive type" effect is very likely confined to a very thin layer somewhat further into the HOPG than the peak of the implantation distribution (Figure 15). Doping with electron acceptors (boron) [72] has not been observed to induce the effect despite their probably having caused less damage (lower mass, lower dose and same energy) to the HOPG than the phosphorus. 
The computed depth profile of the ion-implanted phosphorous in graphite and the computed damage profile are shown in Figure 15, curves 1 and 2. The corresponding computed profiles for the ion-implanted argon in graphite are shown in Figure 33, curves 3 and 4. Since there is no characterized implantation model for the stopping power of HOPG as a substrate we selected the closest substrate material in the library, graphite, for the simulation.

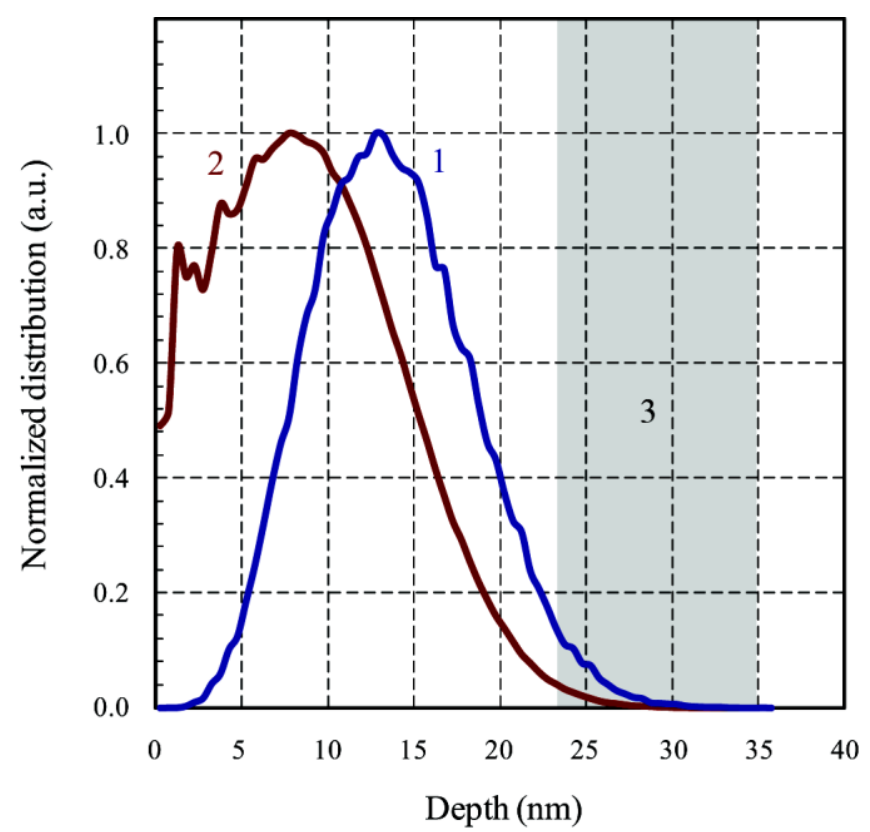

Figure 15: SRIM simulated distribution of (1) phosphorus ions implanted in graphite at $E p=10 \mathrm{keV}$, (2) damage in graphite lattice caused by implant and (3) probable region of effect.

Minimal doping and energy levels were deliberately selected in order to minimize the damage done by the implantation to the graphene sheets in the HOPG. This reduces the disorder in the lattice. This damage could potentially provide scattering centers. These scattering centers would very likely have a negative impact on any electron- 
electron coupling mechanism, regardless of whether the coupling mechanism is phonon or plasmon mediated.

An array species were chosen for ion-implantation doping to provide us with a bigger picture of what dopants were most promising for investigation. Prior to doping, SRIM simulations were performed to characterize their stopping characteristics seen in

Table 4.

Table 4. Stopping and range for ion-implanted species at $10 \mathrm{keV}$ and $5 \mathrm{keV}$ for argon. Green highlights the species that showed features that warrant further investigation.

\begin{tabular}{|c|c|c|c|c|c|c|c|c|c|}
\hline Element & $\begin{array}{c}\text { Atom } \\
\text { Radius } \\
\text { (pm) }\end{array}$ & $\begin{array}{c}\text { Crystal } \\
\text { Radius } \\
\text { (pm) }\end{array}$ & $\begin{array}{c}\text { Ionic } \\
\text { Charge }\end{array}$ & $\begin{array}{c}\text { Valence } \\
\text { Electrons }\end{array}$ & $\begin{array}{c}\text { Atomic } \\
\text { Mass } \\
\text { (amu) }\end{array}$ & $\begin{array}{c}\text { Electron } \\
\text { Donor or } \\
\text { Acceptor? }\end{array}$ & $\begin{array}{c}\text { Projected } \\
\text { Range (A) }\end{array}$ & $\begin{array}{c}\text { Longitudinal } \\
\text { Straggle (A) }\end{array}$ & $\begin{array}{c}\text { Lateral } \\
\text { Straggle } \\
\text { (A) }\end{array}$ \\
\hline Aluminum & 118 & 67.5 & $3+$ & 3 & 27 & acceptor & 149 & 49 & 37 \\
\hline Arsenic & 114 & 72 & $3+$ & 5 & 75 & donor & 104 & 25 & 20 \\
\hline Beryllium & 112 & 59 & $2+$ & 2 & 9 & acceptor & 442 & 171 & 130 \\
\hline Fluorine & 42 & 22 & $7+$ & 7 & 19 & acceptor & 193 & 68 & 50 \\
\hline Lithium & 167 & 90 & $1+$ & 1 & 7 & acceptor & 612 & 234 & 187 \\
\hline Nitrogen & 56 & 30 & $3+$ & 5 & 14 & donor & 230 & 84 & 62 \\
\hline Sodium & 190 & 116 & $1+$ & 1 & 23 & acceptor & 169 & 58 & 43 \\
\hline Sulfur & 88 & 51 & $4+$ & 6 & 32 & donor & 125 & 38 & 30 \\
\hline Phosphorus & 98 & 58 & $3+$ & 5 & 31 & donor & 132 & 41 & 32 \\
\hline Argon & 71 & 71 & $0+$ & 8 & 40 & neutral & 72 & 22 & 17 \\
\hline
\end{tabular}

Each dopant was ion-implanted at a low dose $\left(1.2 \times 10^{8} \mathrm{~cm}^{-2}\right)$ and on a separate HOPG sample with a high dose $\left(1.2 \times 10^{11} \mathrm{~cm}^{-2}\right)$ using an energy of $10 \mathrm{keV}$ and current of 104 pA. Argon was used to create columnar damage, which will be seen in sample 023 
and was implanted at $5 \mathrm{keV}$ to give it a shallower implantation depth than the previous implanted phosphorus.

Table 5. Dose and concentration for ion-implanted species. All were done at $10 \mathrm{keV}$ with the exception of argon which was done at $5 \mathrm{keV}$. All used a current of $104 \mathrm{pA}$. Green highlights the species that showed features that warrant further investigation.

\begin{tabular}{|c|c|c|c|c|}
\hline Element & $\begin{array}{c}\text { Low Dose } \\
(\text { ions/cm })\end{array}$ & $\begin{array}{c}\text { High Dose } \\
\left(\text { ions/cm }^{2}\right)\end{array}$ & $\begin{array}{c}\text { Concentration } \\
\text { at Low Dose } \\
\text { (atoms/cm }\end{array}$ & $\begin{array}{c}\text { Concentration } \\
\text { at High Dose } \\
\text { (atoms/cm }\end{array}$ \\
\hline Aluminum & $1.20 \mathrm{E}+08$ & $1.20 \mathrm{E}+11$ & $9.8 \mathrm{E}+13$ & $9.8 \mathrm{E}+16$ \\
\hline Arsenic & $1.20 \mathrm{E}+08$ & $1.20 \mathrm{E}+11$ & $1.92 \mathrm{E}+14$ & $1.92 \mathrm{E}+17$ \\
\hline Beryllium & $1.20 \mathrm{E}+08$ & $1.20 \mathrm{E}+11$ & $2.81 \mathrm{E}+13$ & $2.81 \mathrm{E}+16$ \\
\hline Fluorine & $1.20 \mathrm{E}+08$ & $1.20 \mathrm{E}+11$ & $7.06 \mathrm{E}+13$ & $7.06 \mathrm{E}+16$ \\
\hline Lithium & $1.20 \mathrm{E}+08$ & $1.20 \mathrm{E}+11$ & $2.05 \mathrm{E}+13$ & $2.05 \mathrm{E}+16$ \\
\hline Nitrogen & $1.20 \mathrm{E}+08$ & $1.20 \mathrm{E}+11$ & $5.71 \mathrm{E}+13$ & $5.71 \mathrm{E}+16$ \\
\hline Sodium & $1.20 \mathrm{E}+08$ & $1.20 \mathrm{E}+11$ & $8.28 \mathrm{E}+13$ & $8.28 \mathrm{E}+16$ \\
\hline Sulfur & $1.20 \mathrm{E}+08$ & $1.20 \mathrm{E}+11$ & $1.26 \mathrm{E}+14$ & $1.26 \mathrm{E}+17$ \\
\hline Phosphorus & $1.20 \mathrm{E}+08$ & $1.20 \mathrm{E}+11$ & $1.17 \mathrm{E}+14$ & $1.17 \mathrm{E}+17$ \\
\hline Argon & $1.20 \mathrm{E}+08$ & None & $1.45 \mathrm{E}+14$ & None \\
\hline
\end{tabular}

Since phosphorus showed the best results (which will be talked about and shown later in the Results section) and was the easiest for us to work with, many other ionimplantations were performed. One was a multi implantation method that used 5 different energies shown in Table 6. This was performed from highest energy to lowest so that the deepest penetration energy does not have to cross through a prior doped section. These were done with a low dose $\left(1.2 \times 10^{8} \mathrm{~cm}^{-2}\right)$ and current of $104 \mathrm{pA}$. 
Table 6. Multi-energy implantation of phosphorus for sample 065.

\begin{tabular}{|c|c|c|c|c|c|}
\hline Sample & $\begin{array}{c}\text { Energy 1 } \\
(\mathbf{k e V})\end{array}$ & $\begin{array}{c}\text { Energy 2 } \\
(\mathbf{k e V})\end{array}$ & $\begin{array}{c}\text { Energy 3 } \\
(\mathbf{k e V})\end{array}$ & $\begin{array}{c}\text { Energy 4 } \\
(\mathbf{k e V})\end{array}$ & $\begin{array}{c}\text { Energy 5 } \\
(\mathbf{k e V})\end{array}$ \\
\hline 065 & 20 & 10 & 6 & 3 & 1 \\
\hline
\end{tabular}

The doped-while-grown material was prepared in a proven graphene Chemical Vapor Deposition system using plasma enhanced Chemical Vapor Deposition on HOPG substrates. The HOPG was used as a seed crystal as it was easier to remove the dopedwhile-grown layer from the HOPG via exfoliation than a monolayer from copper foil. The gas mixture was methane at $20 \mathrm{sccm}$, hydrogen at $10 \mathrm{sccm}$, argon at $14 \mathrm{sccm}$ and $0.1 \%$ phosphine in an argon carrier gas at $5 \mathrm{sccm}$. The plasma power was $30 \mathrm{~W}$ for 15 minutes as seen in Table 7. The substrate temperature was $800{ }^{\circ} \mathrm{C}$ and two pieces of partially overlapping copper film were placed adjacent to the HOPG in order to provide a visual witness that graphene had been grown as seen in Figure 16.

Table 7. Plasma Enhanced CVD recipe. Phosphine used was $1000 \mathrm{ppm}$ in argon.

\begin{tabular}{|c|c|c|c|c|c|c|c|}
\hline \multicolumn{4}{|c|}{ Flow Rate ( sccm ) } & Plasma & $\begin{array}{c}\text { Deposition } \\
\text { Power } \\
\text { Time } \\
(\mathbf{~ s ~})\end{array}$ & $\begin{array}{c}\text { Deposition } \\
\text { Temperature } \\
\left({ }^{\circ} \mathbf{C}\right)\end{array}$ & $\begin{array}{c}\text { Annealing } \\
\text { Temperature } \\
\left({ }^{\circ} \mathbf{C}\right)\end{array}$ \\
\hline 20 & 10 & 14 & 5 & 30 & 900 & 800 & 900 \\
\hline
\end{tabular}

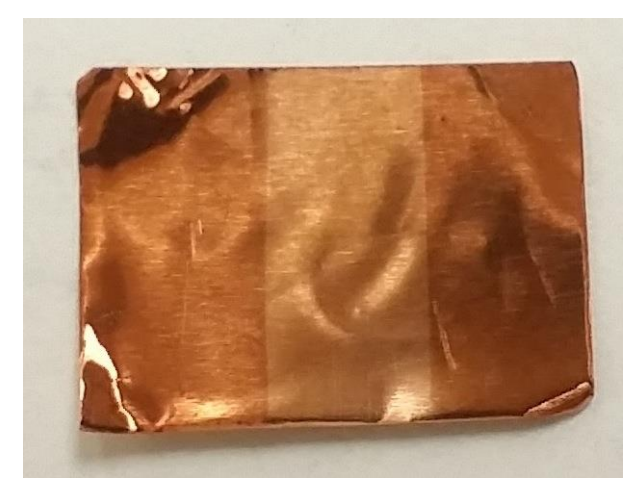

Figure 16: Copper witness visually showing graphene after successful Chemical Vapor Deposition process. 


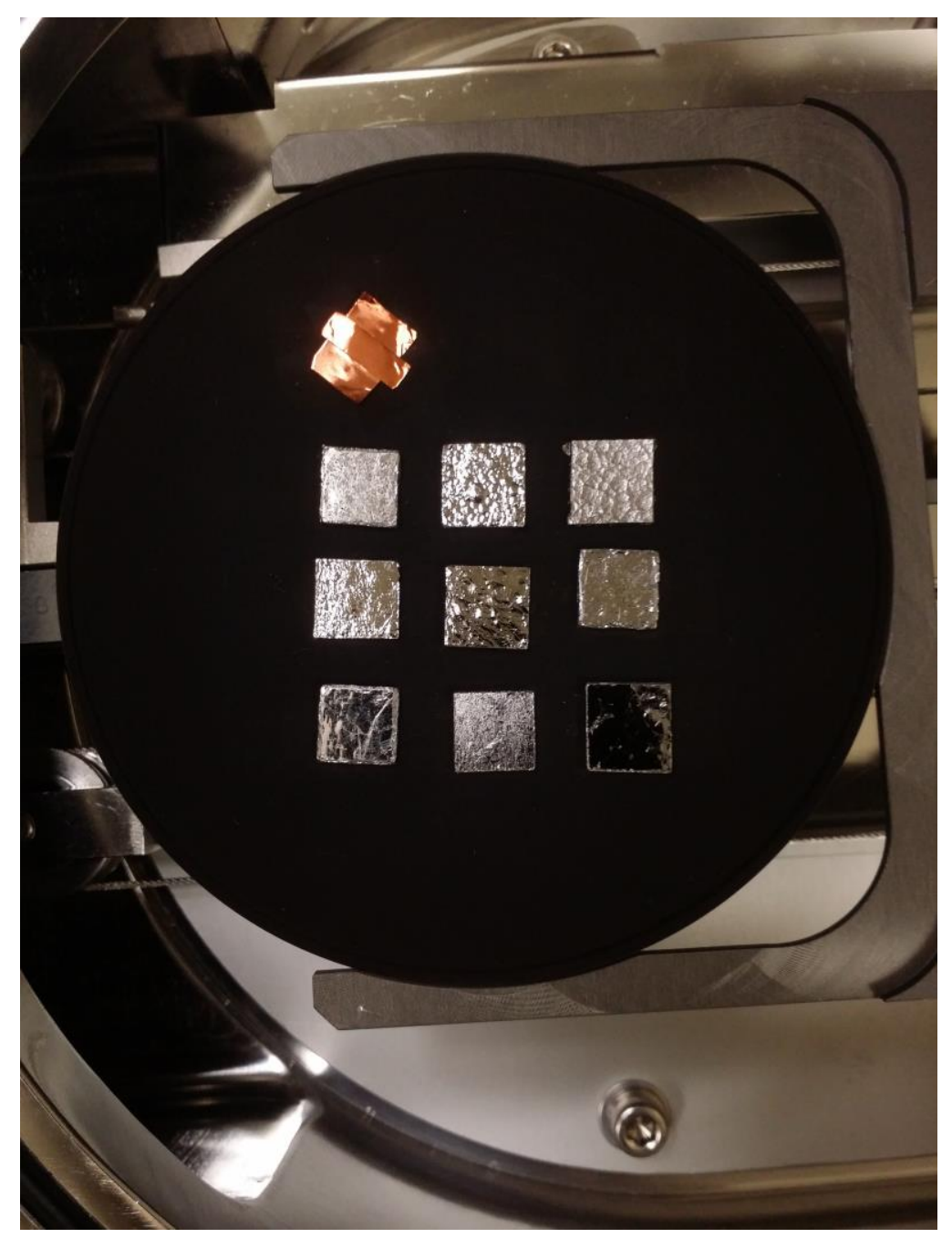

Figure 17: Graphite sample holder with array of HOPG samples loaded. Two copper witness samples are placed at the upper left corner of the array to indicate the position of the first sample in the series.

After doping, either by implantation or through growth, the sample was placed in the closed cycle refrigeration system again. The $R$ vs. $T$ characteristic of the HOPG was then re-measured. All measurements were averaged delta mode in nature and each data 
point recorded was the result of 250 individual measurements averaged together. Measurement speed is approximately 10 seconds per final averaged data point recorded. All measurements were made at the minimum practical stimulus current, always $10 \mu \mathrm{A}$ or less (typically $1 \mu \mathrm{A})$, to avoid current induced degradation of the responses.

After the measuring of the $R$ vs. $T$ characteristic of the post doped bulk sample the doped surface was exfoliated using silicon adhesive Kapton ${ }^{\circledR}$ film tape to remove multilayers of graphene for testing. These multilayer graphene films, still on the tape, were then placed into the closed cycle refrigeration system and the $R$ vs. $T$ characteristic of the graphene multilayer stack was measured. To check that the graphene exfoliations were affected by an applied magnetic field similarly to the bulk phosphorous ionimplanted HOPG shown in Figure 22 a modest, calculated, 100 to 350 milli-Tesla magnetic field was supplied by a dc driven coil placed externally over the refrigerator vacuum shroud and the $R$ vs. $T$ characteristic was re-measured on a number of samples. In addition to the $R$ vs. $T$ measurements, several samples were tested in SQUID magnetometer and AC susceptometer. The results of these measurements and details of Hall effect measurements on one of our exfoliated doped-while-grown samples and a Raman characterization of the film are presented in the results section below.

Estimating the thickness of graphene can be roughly done by measuring the light transmission of a monolayer of the material. As a guide, the thickness for of the material can be determined as $T=(.977){ }^{\mathrm{n}}$ for $\mathrm{n}$ layers. Other considerations, such as the film tape transmission, must be made which adds more uncertainty to the estimation method. In order to obtain more accurate information as to the thickness of the exfoliated layers bound to the adhesive backing of the tape, Raman spectroscopy was performed. This was 
done at both room temperature and $T=77 \mathrm{~K}$ on different positions on the exfoliated material. Room temperature van der Pauw and Hall measurement were performed in an attempt to determine the resistivity of the Phosphine-doped Graphene; as well as the $\mathrm{n}_{2 \mathrm{D}}$ density and mobility values of the material.

\section{E. Susceptometer}

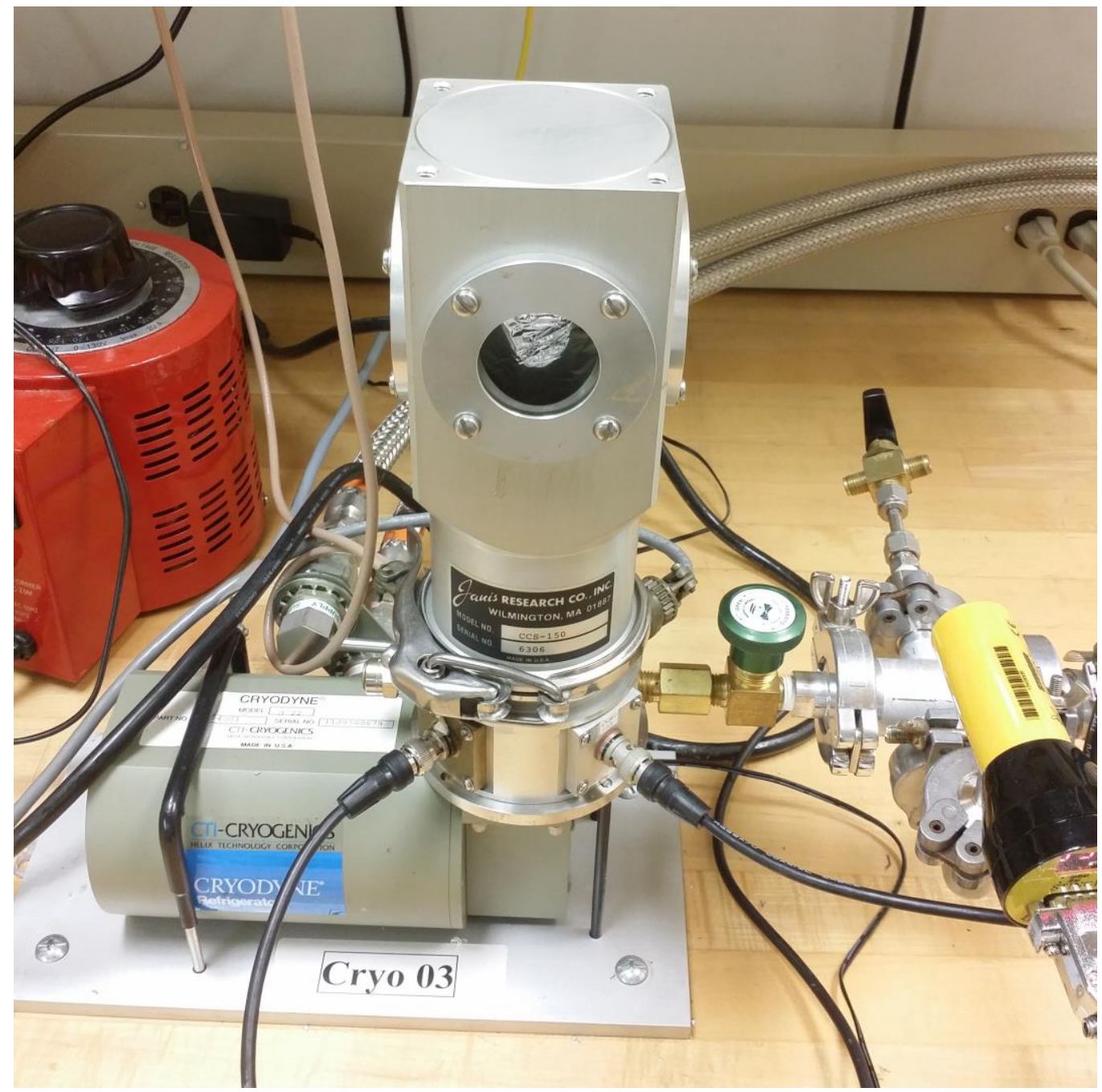

Figure 18: Susceptometer System. 


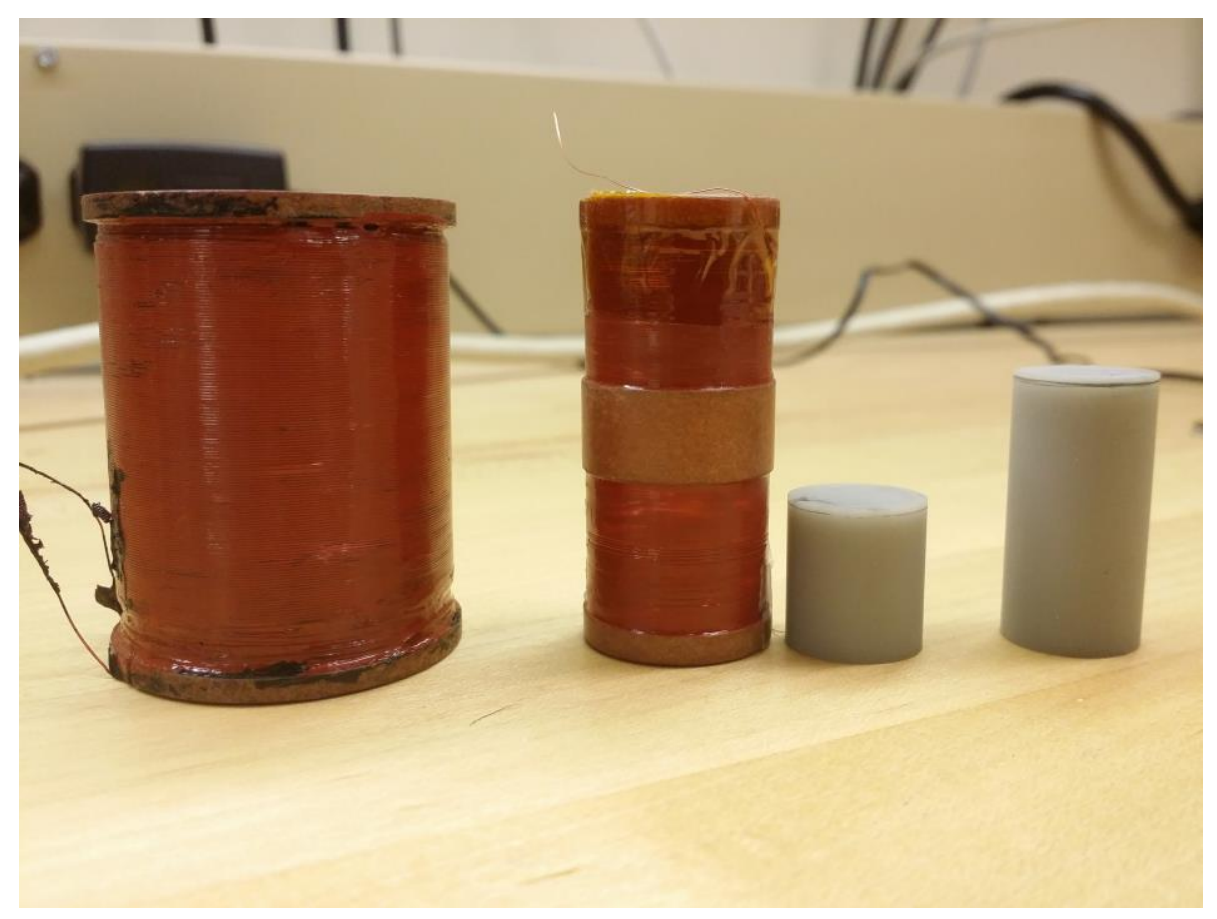

Figure 19: Outer coil, inner coil and 2 pieces of single bore alumina.

In Figure 19 we see coils used for susceptometer. On the left, is the outer coil that is responsible for delivering the magnetic field. All turns are in the same direction. Inner coil, on the right, is used for detecting the magnetic field. These have half the windings going in one direction, and the other half going in the opposite direction. This cancels out the field being received and should give a remaining voltage representative of the Meissner effect experienced by the sample. Leads exit on the top.

The Alumina was chosen for its high thermal conductivity, while also being an electric insulator. This ensures that the sample is lowered to the appropriate temperature while also preventing shunting of superconductivity. The diameter allows them to fit into the inner coil and rest the sample between the two pieces of alumina. The position of the sample is adjusted so that it is located in the center of one of the winding directions (so 
located either $1 / 4$ or $3 / 4$ of the height of the inner coil). The Alumina is raised and lowered accordingly, using a spacer.

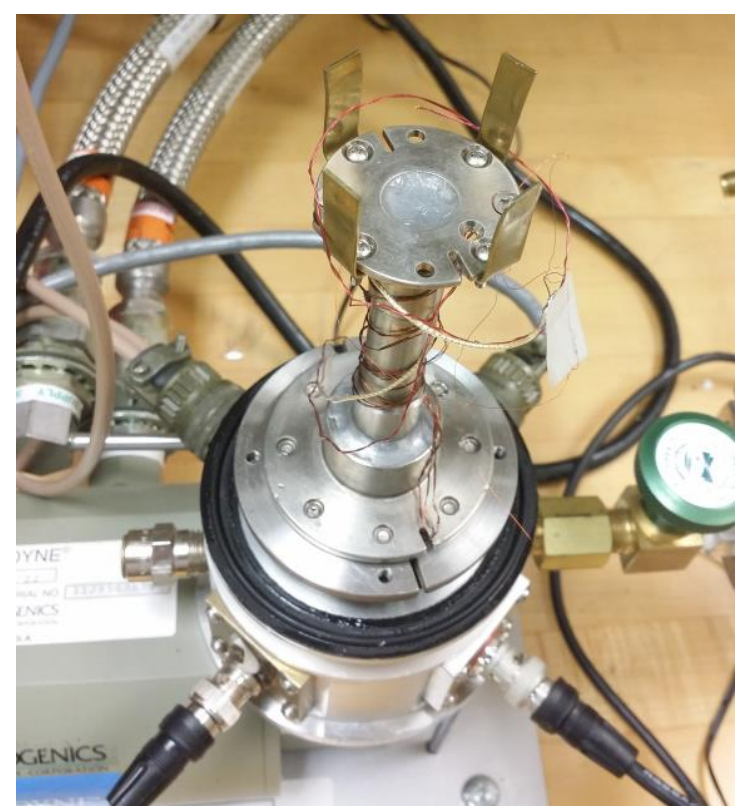

Figure 20: Brass guides, custom made to center coils. These are screwed on top of the coldhead.

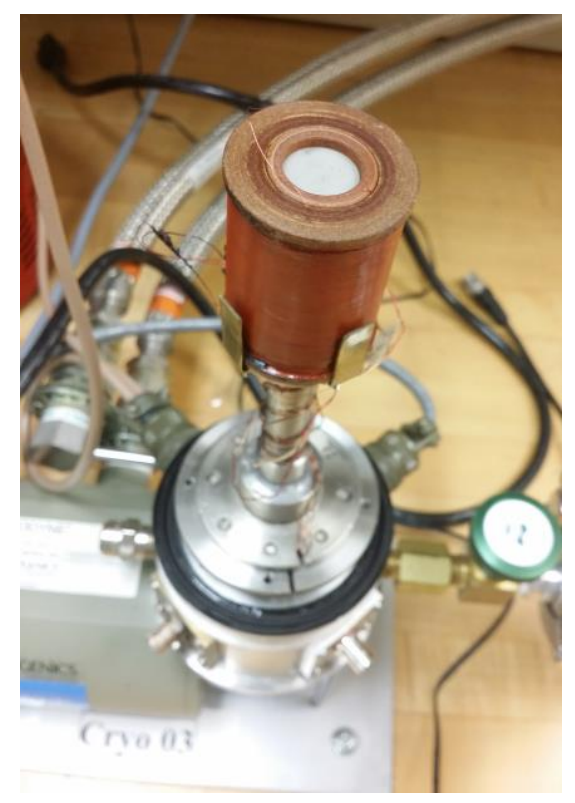

Figure 21: Outer coil, inner coil, alumina with sample between alumina pieces and centered using custom sample holder are placed on top of the coldhead and held in placed by 4 brass guides that wedge the assembly in place. 
The complete assembly was covered by an aluminum shroud that was wrapped 6 times in aluminized mylar. The final aluminum shield was placed over this to create the vacuum seal. A Signal Recovery Model 7270 DSP Lock-in amplifier was used to create the signal to the outer coil as well as measure using the inner coil. LabView software was created to control all functions of this system (see Appendix 25 to Appendix 37 ).

To see if the system was working correctly, we used a $\mathrm{YBa}_{2} \mathrm{Cu}_{3} \mathrm{O}_{7-\mathrm{x}}$ Hairpin sample since this is a known superconductor. We found that a reliable reading could not be yielded, after numerous attempts to balance the coils and find the lowest noise frequency.

\section{RESULTS}

Phosphorous-implanted HOPG samples were shown in prior work [72] to have exhibited deviations from the expected rise in resistance as the temperature is reduced to some point above $100 \mathrm{~K}$ (Figure 22). The relatively large drop in resistance at lower temperatures (by a factor of more than 2) was also considered a possible indication of superconductivity in the sample. It was also noted that the application of a modest magnetic field reversed this trend. All of this as well as the resistance vs. temperature curve for non-doped HOPG are shown in Figure 22 overleaf. 


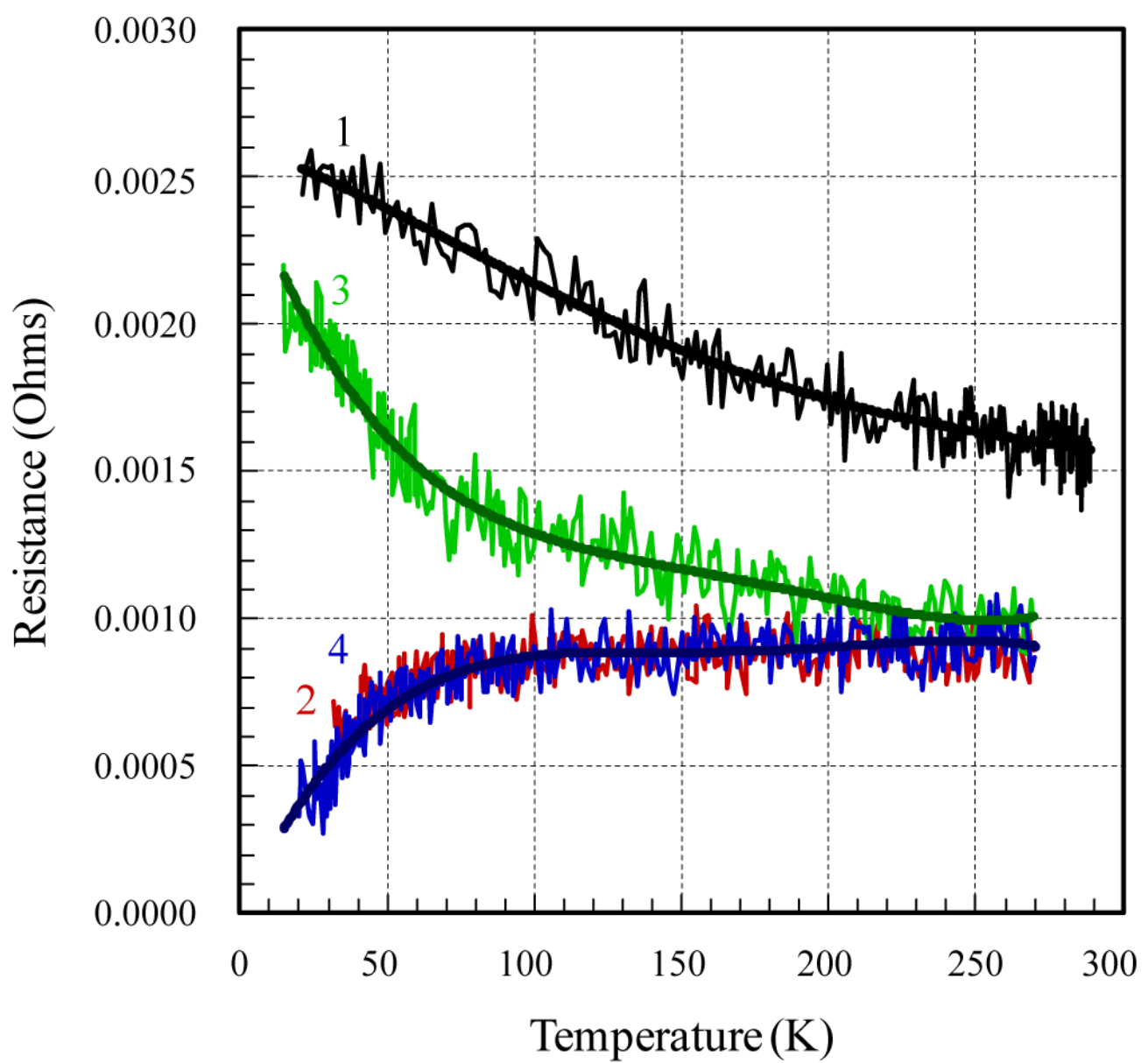

Figure 22: Measured $R$ vs. $T$ dependence of a phosphorous-implanted HOPG sample. Curve 1 (black) is before implantation, curve 2 (red) is of the phosphorousimplanted sample before magnetic field was applied, curve 3 (green) is of the phosphorous-implanted sample with magnetic field applied and curve 4 (blue) is of the phosphorous-implanted sample after the magnetic field applied in curve 3 magnetic field was removed.

The lack of zero resistance and the modest magnetic field (maximum attainable was less than $0.035 \mathrm{~T}$ ) required to quench the effect even in the exfoliated multilayer graphene samples is shown in for a representative sample. 


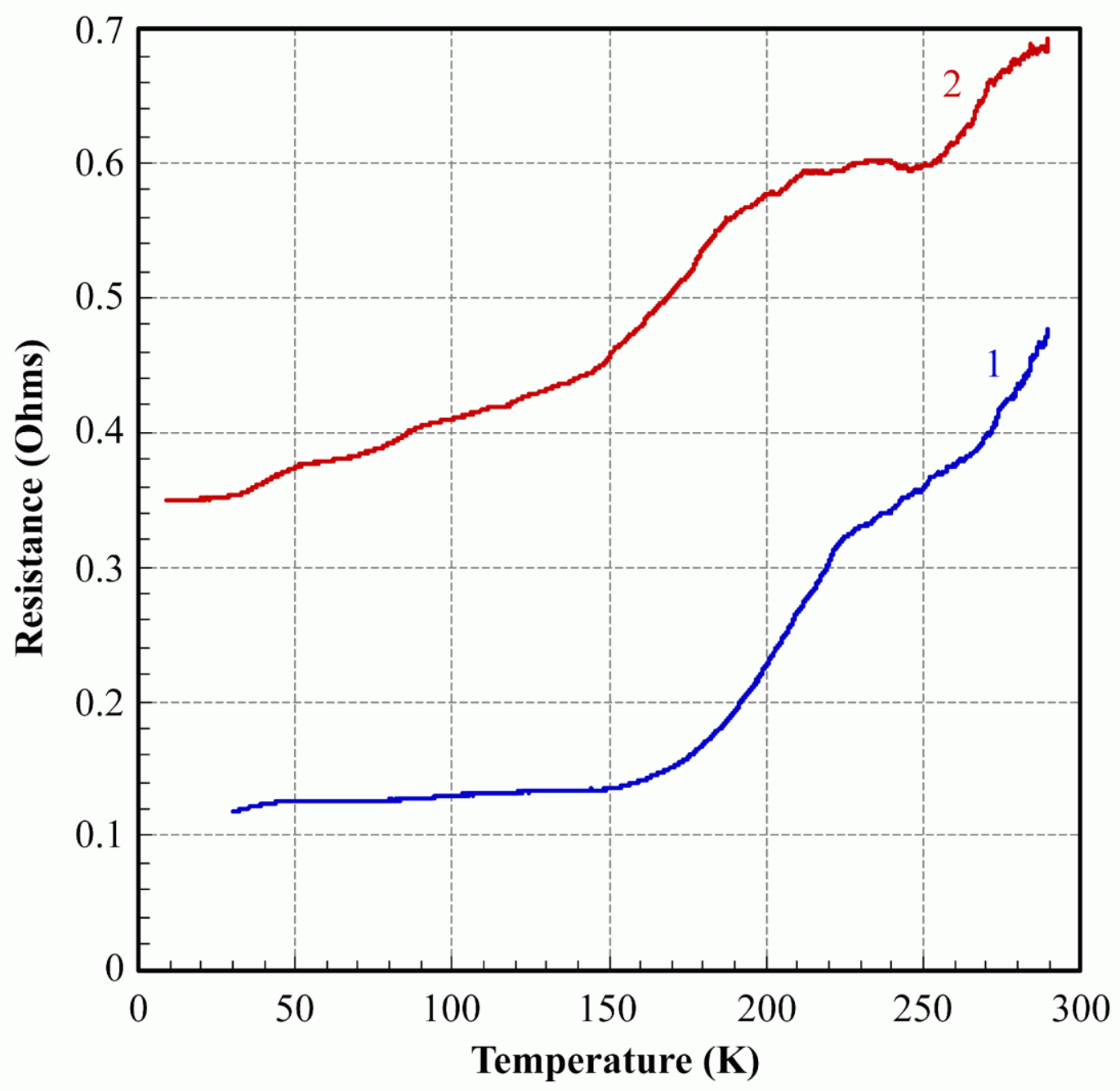

Figure 23: $R$ vs. $T$ of thin film exfoliated from phosphorous-doped HOPG measured without (1) and with $0.035 \mathrm{~T}$ applied magnetic field (2). 


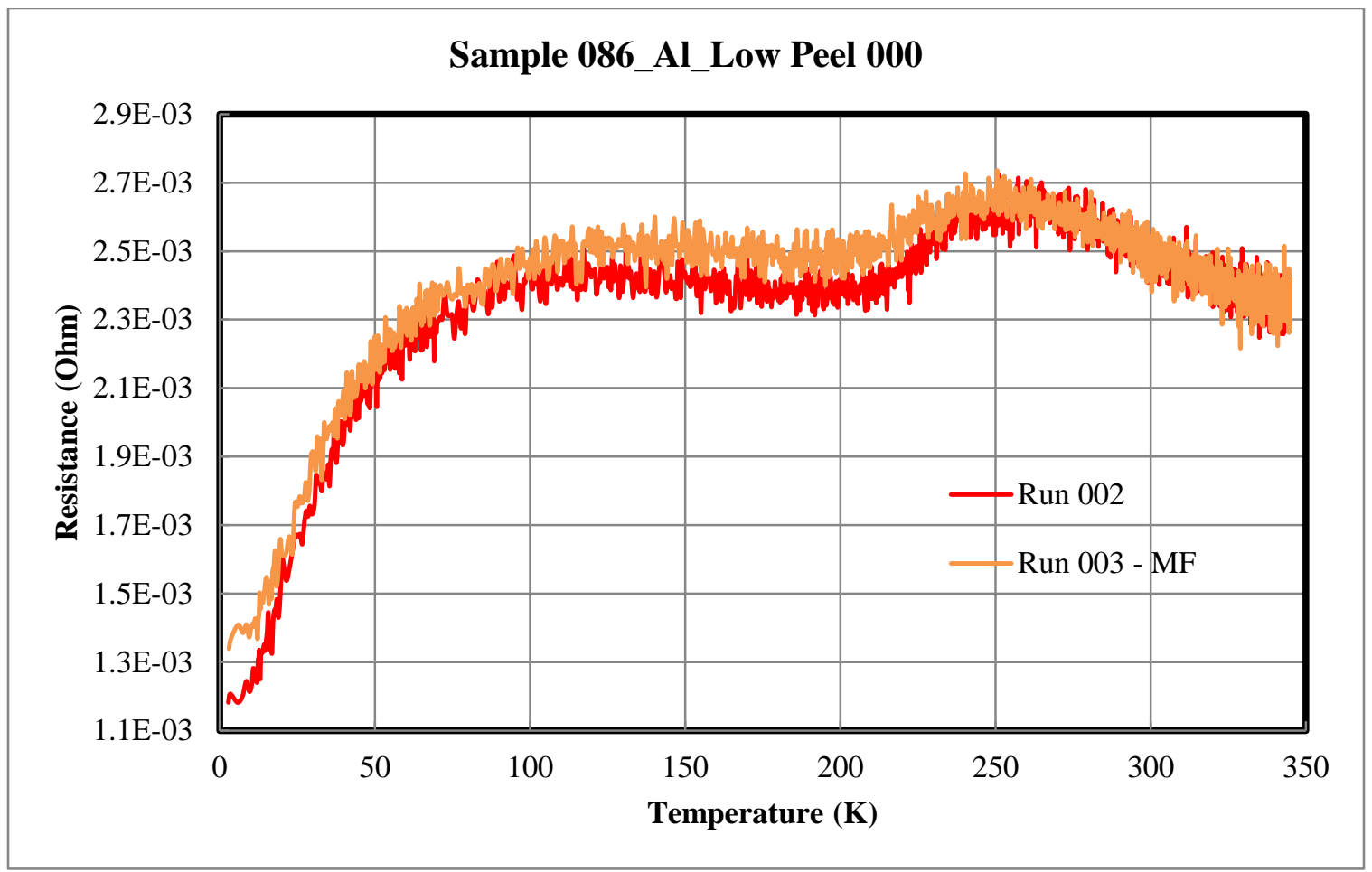

Figure 24. Aluminum ion-implanted sample. Bulk HOPG with low dose.

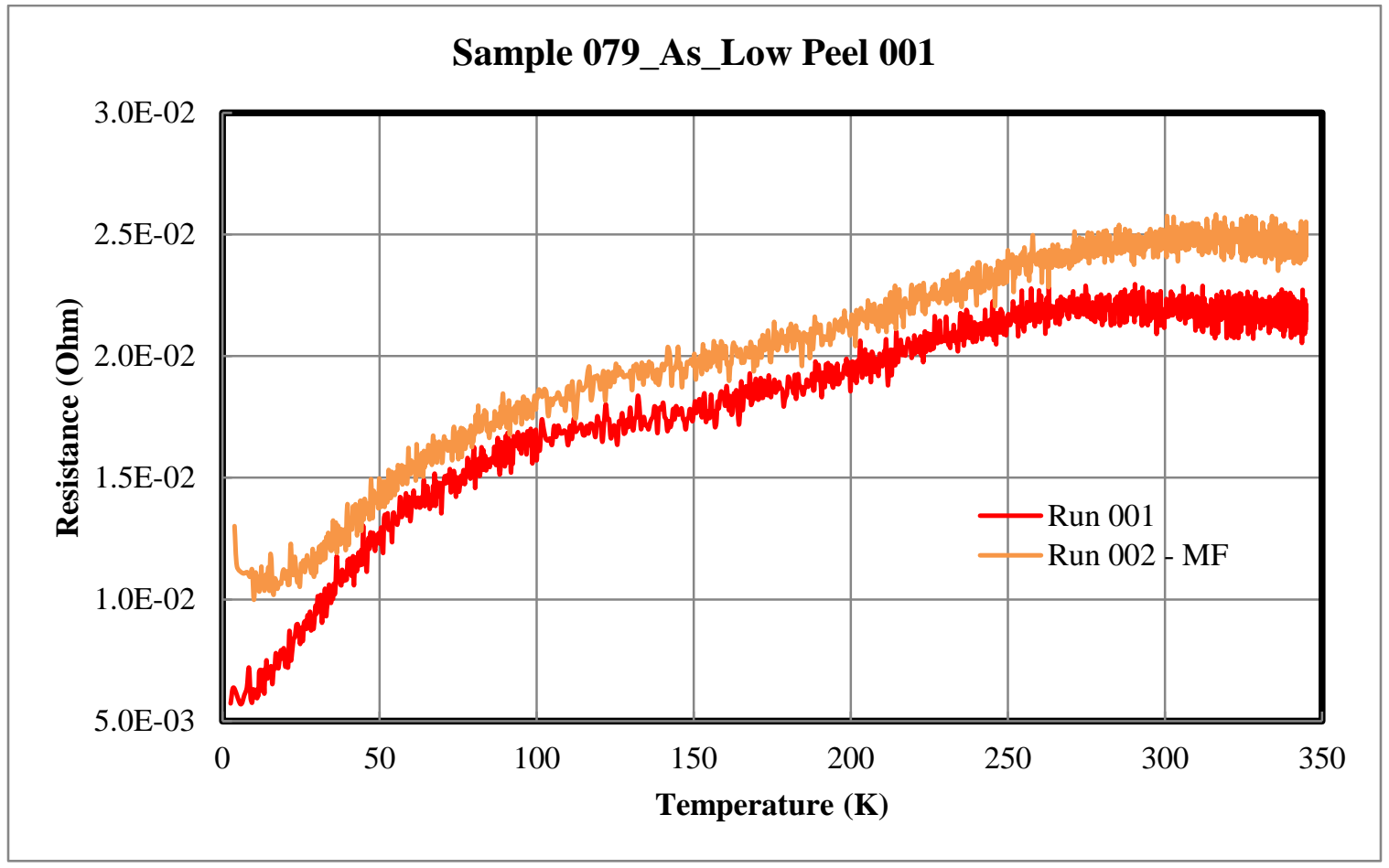

Figure 25. Arsenic ion-implanted sample. Peel with low dose. 


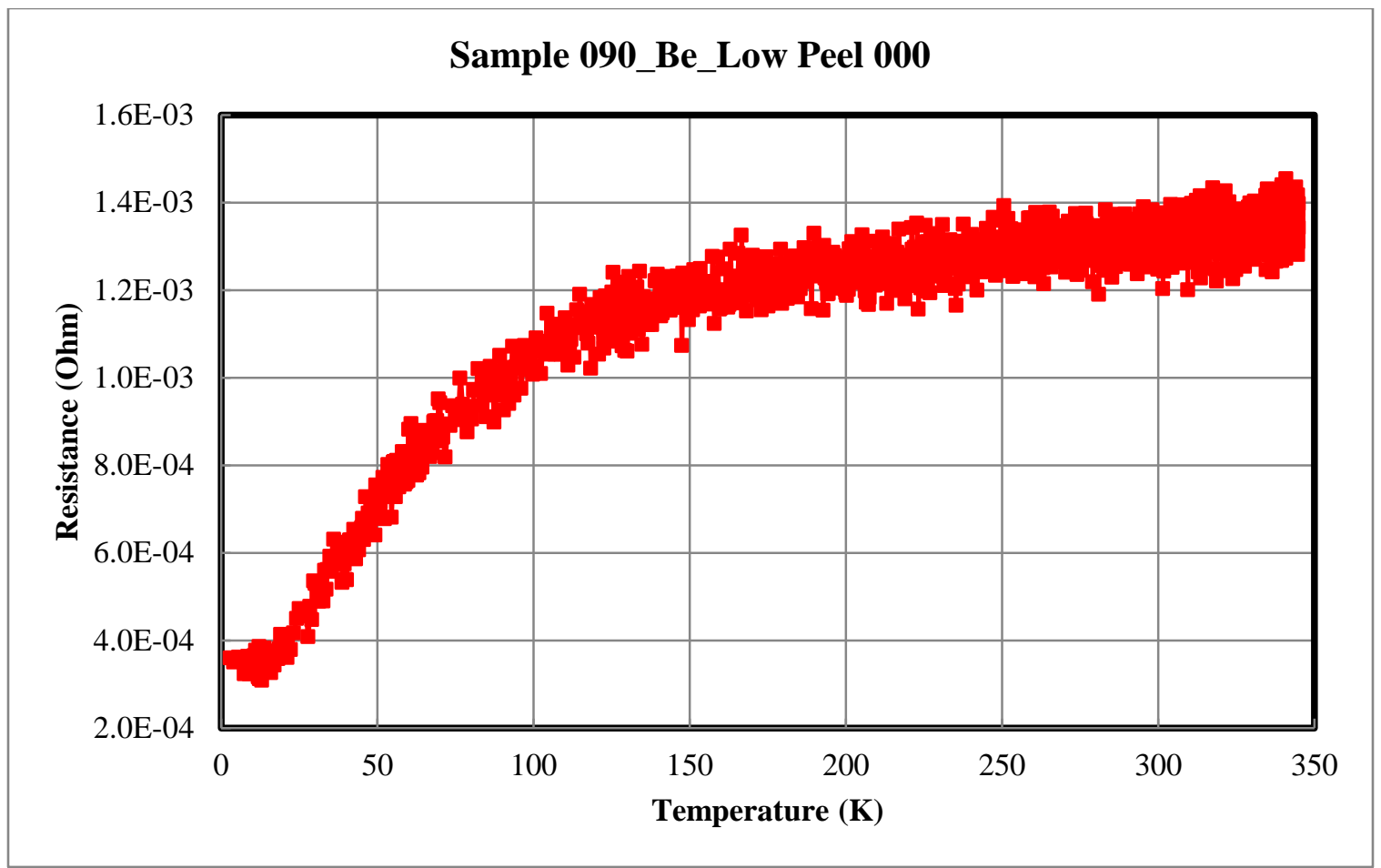

Figure 26. Beryllium ion-implanted sample. Bulk with low dose.

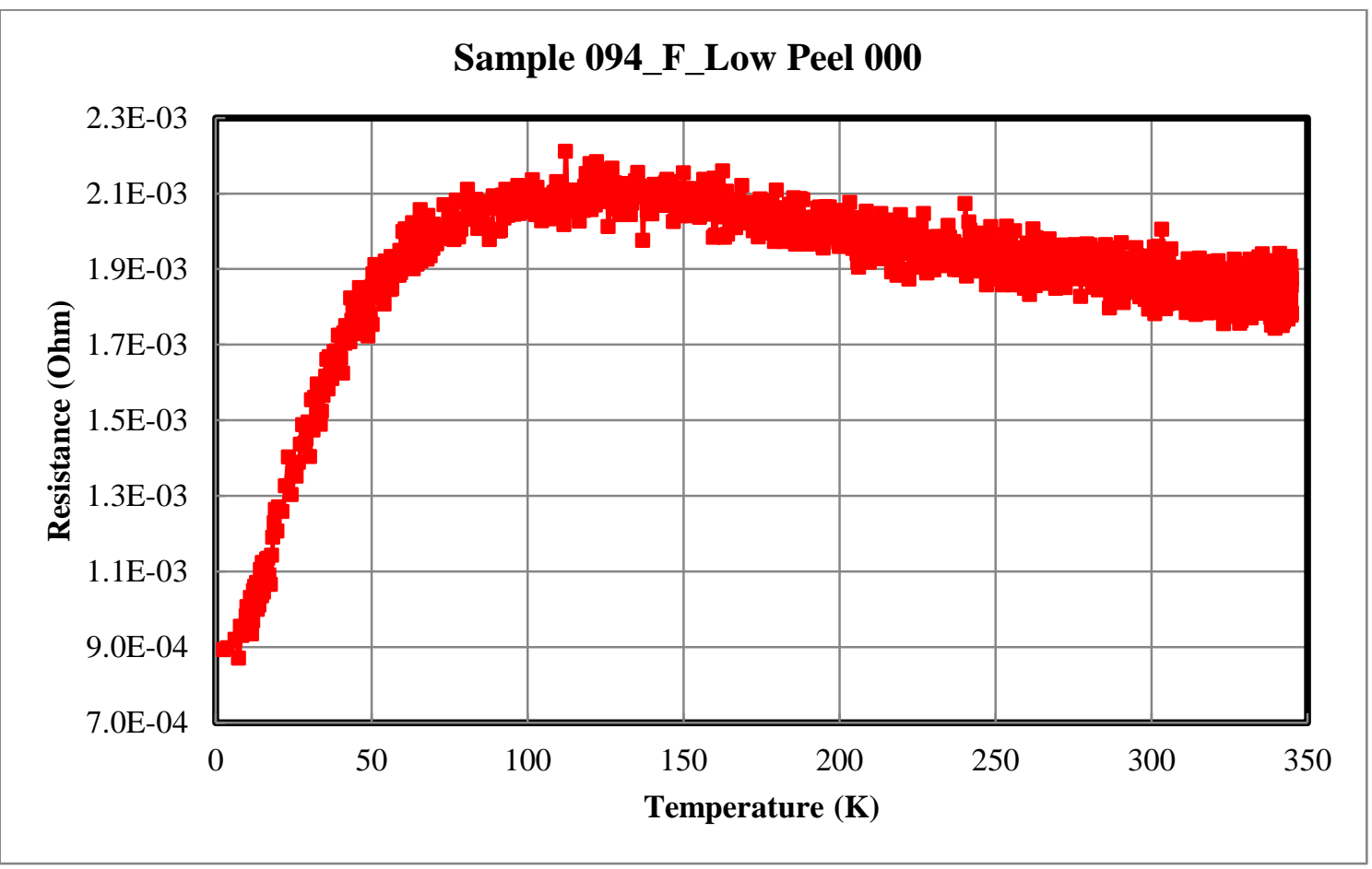

Figure 27. Fluorine ion-implanted sample. Bulk with low dose. 


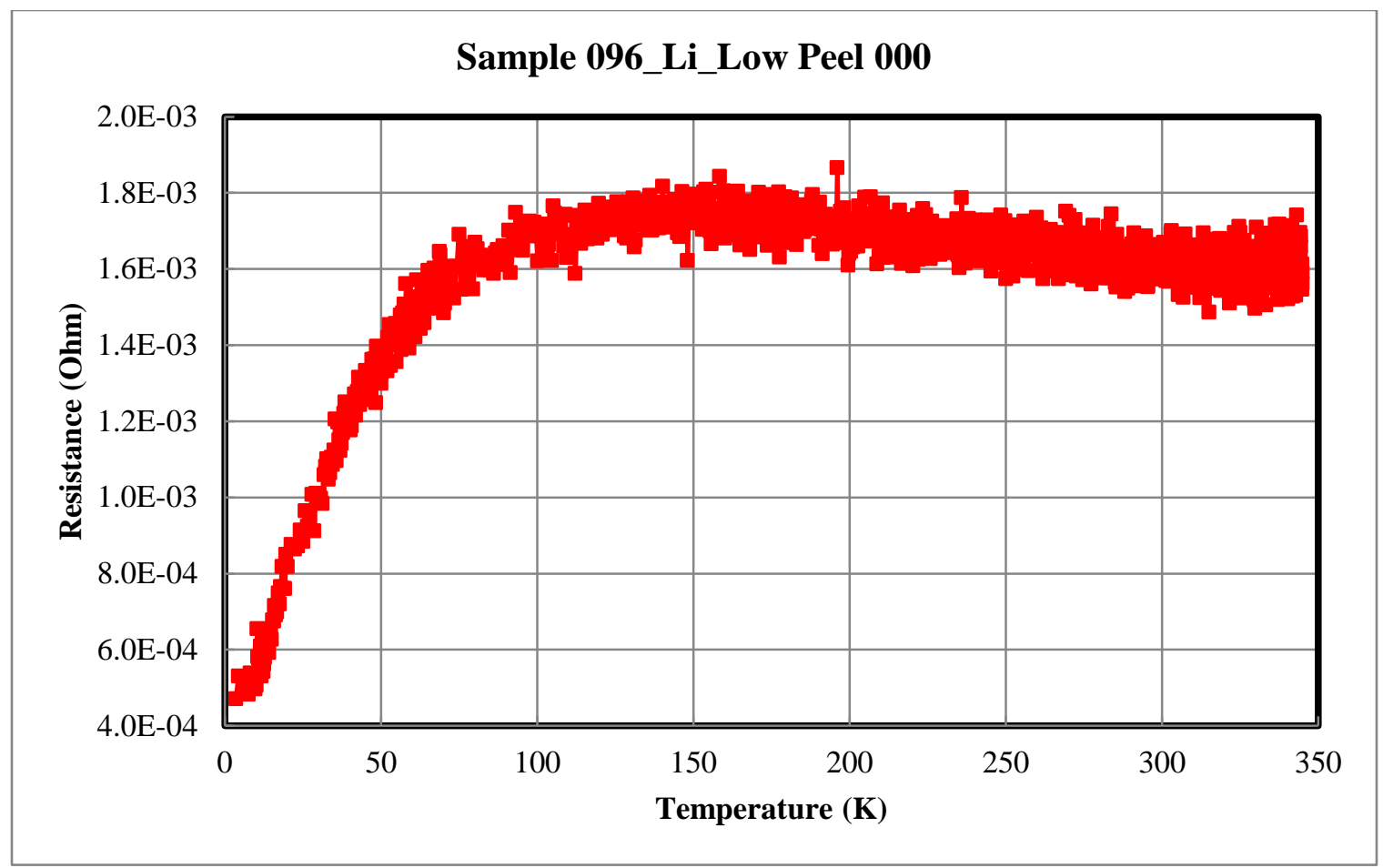

Figure 28. Lithium ion-implanted sample. Bulk with low dose.

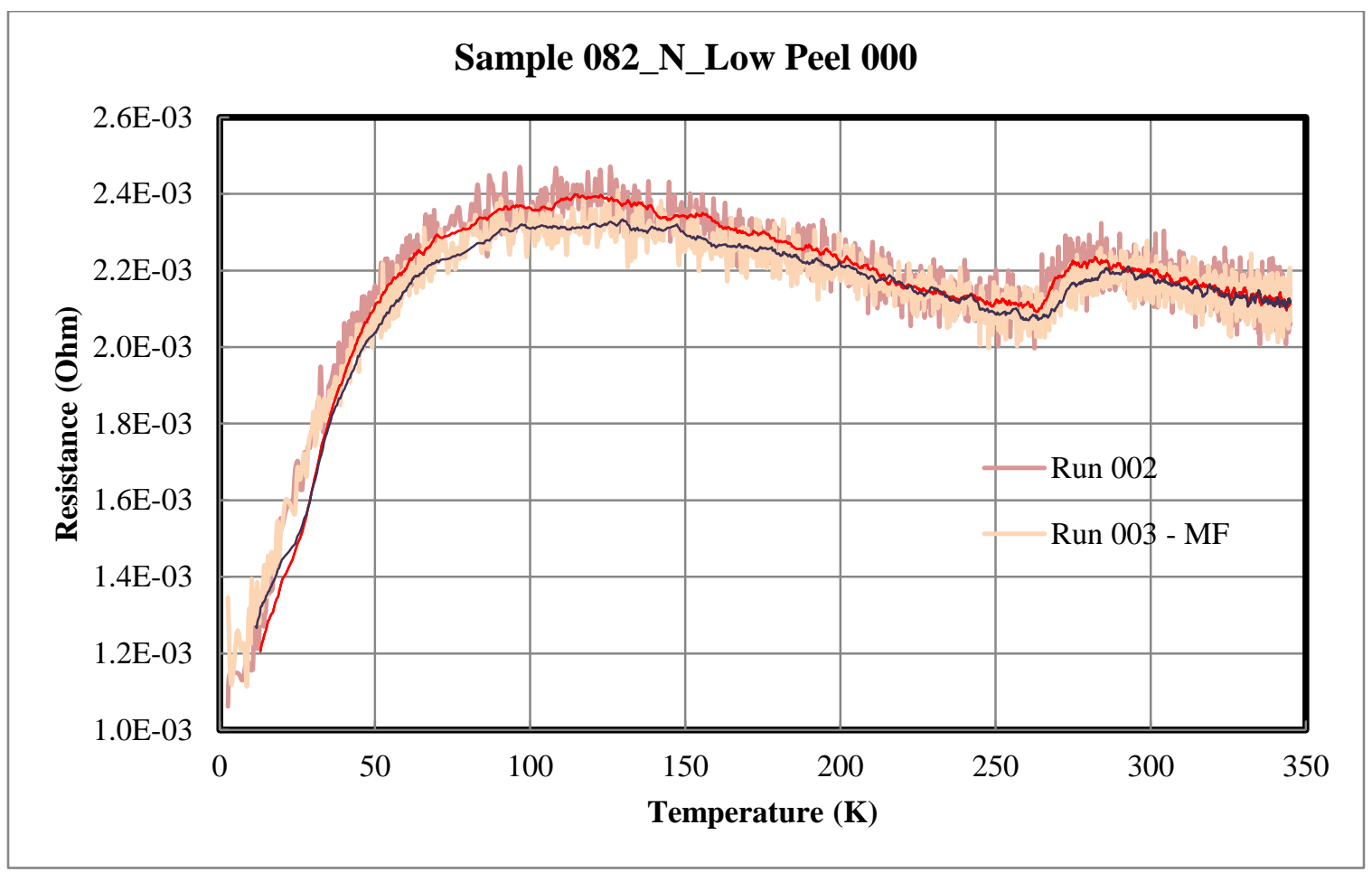

Figure 29. Nitrogen ion-implanted sample. Bulk with low dose. 


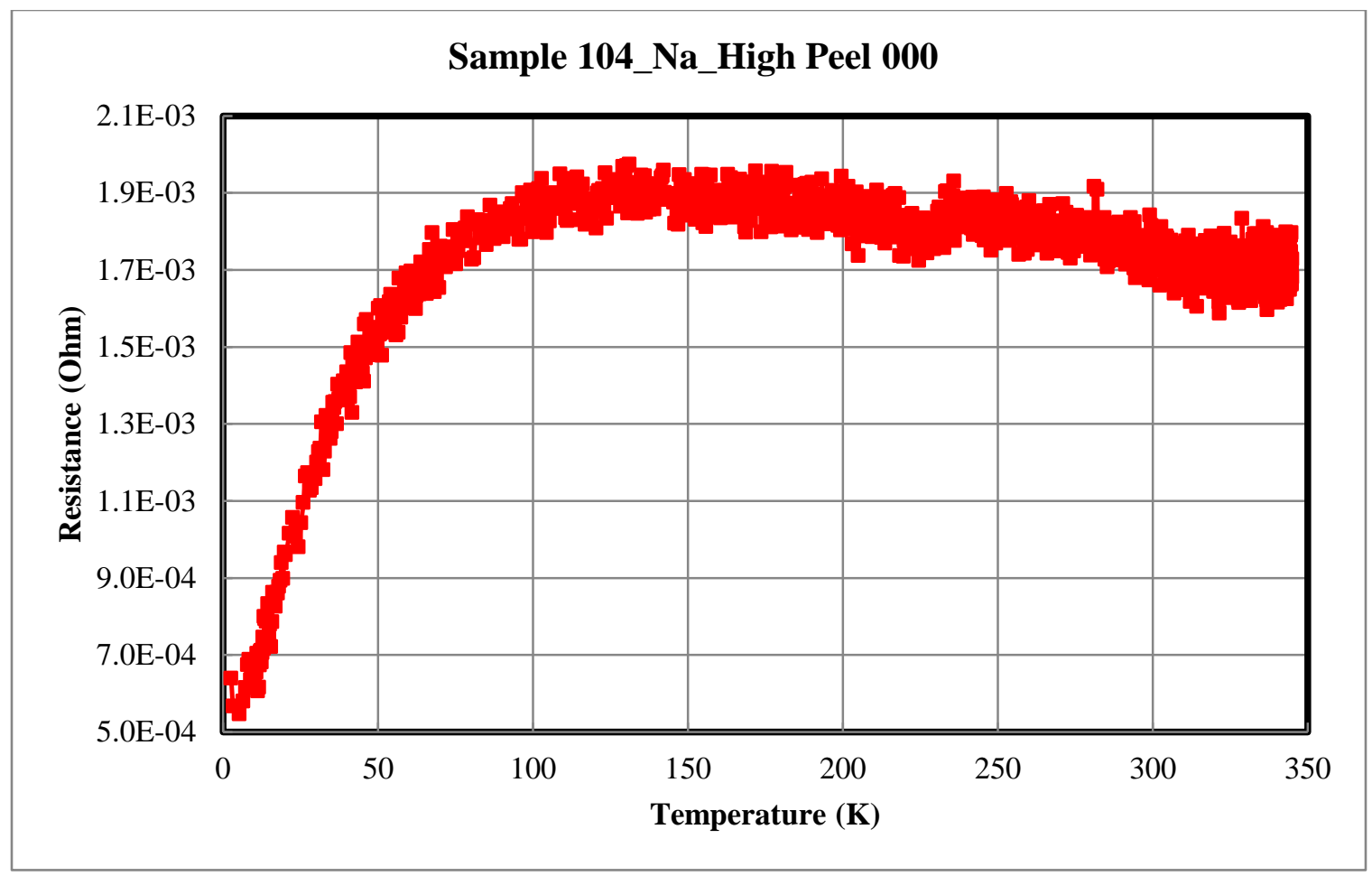

Figure 30. Sodium ion-implanted sample. Bulk with low dose.

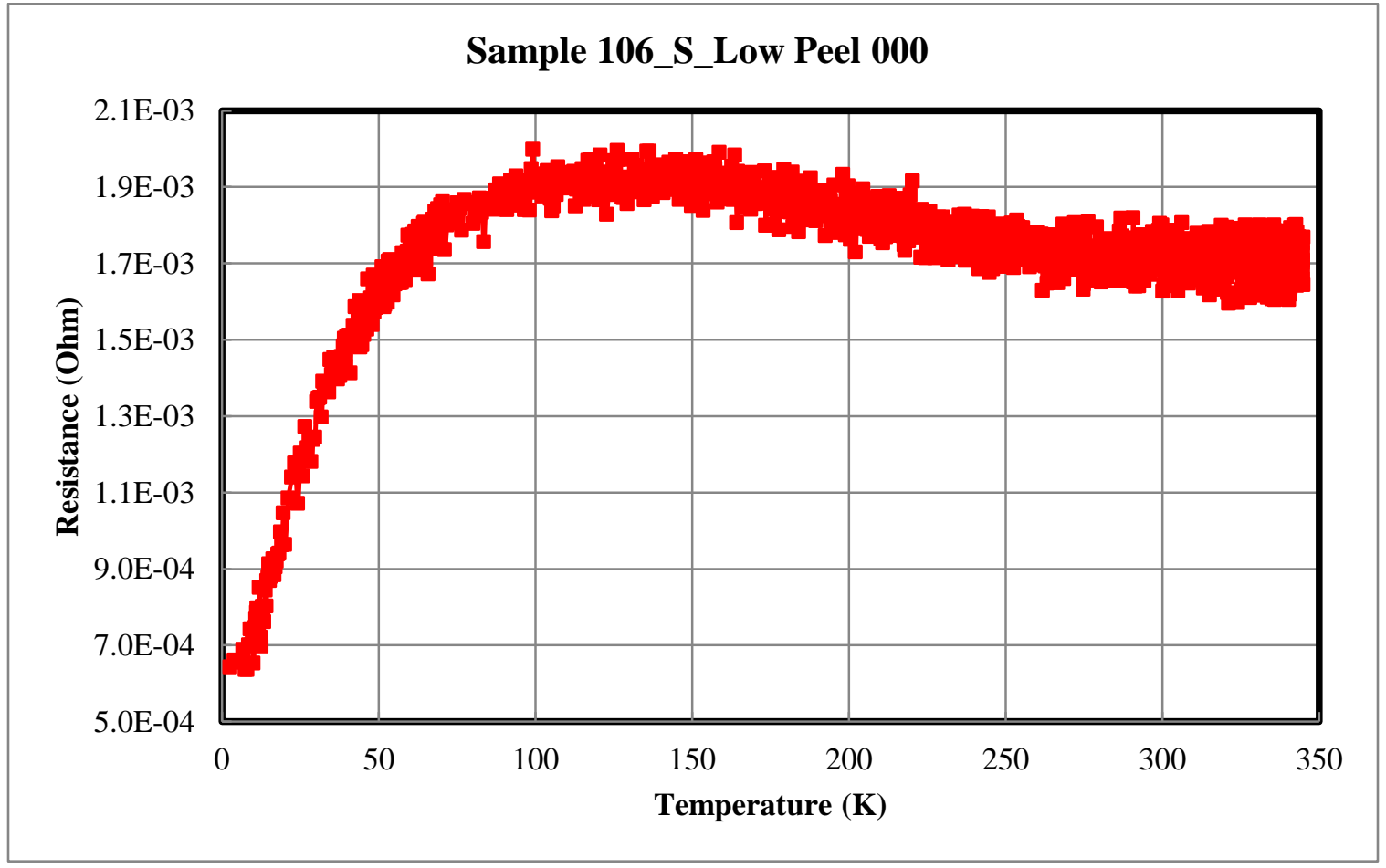

Figure 31. Sulfur ion-implanted sample. Bulk with low dose. 
Beryllium, fluorine, lithium and sulfur did not show any features at high or low doses (see Appendix 15 -Appendix 20, Appendix 23 , Appendix 24 ). Aluminum showed features for both low and high while sodium showed features only at high doses (see Appendix 7 -Appendix 9, Appendix 21, Appendix 22). All of the pentavalent species (arsenic, nitrogen, phosphorus) showed features (Appendix 5, Appendix 6 , Appendix 10 -Appendix 14 ). Since phosphorus showed the strongest features and was also the easiest to work with, more in depth research was focused on it.

In order to better understand the potential causes for the observed results, a number of $R$ vs. $T$ characteristics are examined, shown in Figure 32, of similarly exfoliated graphene films taken from bulk phosphorous-implanted HOPG samples.

Comparing characteristics (a)-(d) in Figure 32 it is clear that there is a step in resistance at a temperature of approximately 50-60 K in all of the samples. Upon close examination of the data, it can be determined that there is a second resistance step at 100$120 \mathrm{~K}$, a third at a temperature range of approximately $150-180 \mathrm{~K}$ and, yet a fourth at a temperature from about 200-240 K.

Additional steps can be observed in the $R$ vs. $T$ characteristics of just about all of the samples. The most probable explanation is that the features are due to magnetic vortex lattice melting and subsequent flux-flow losses. 


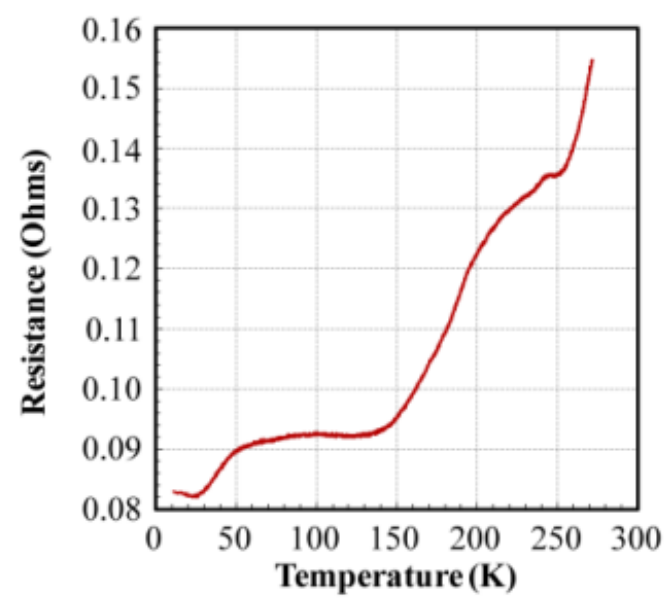

a

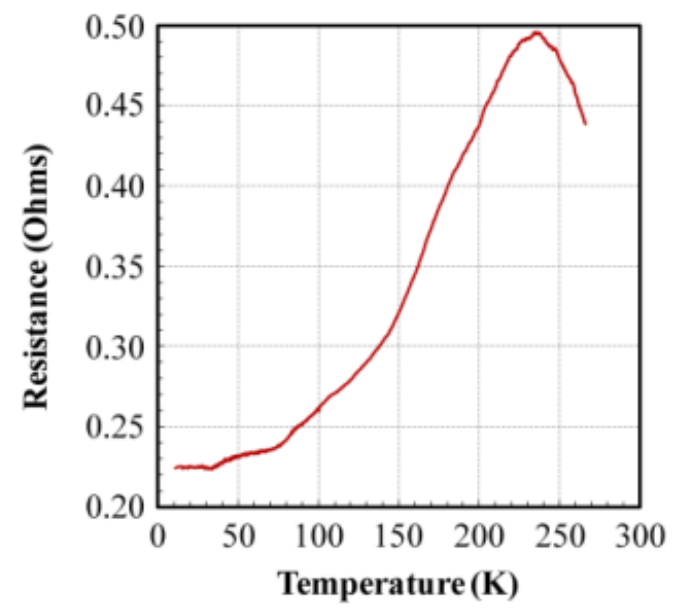

C

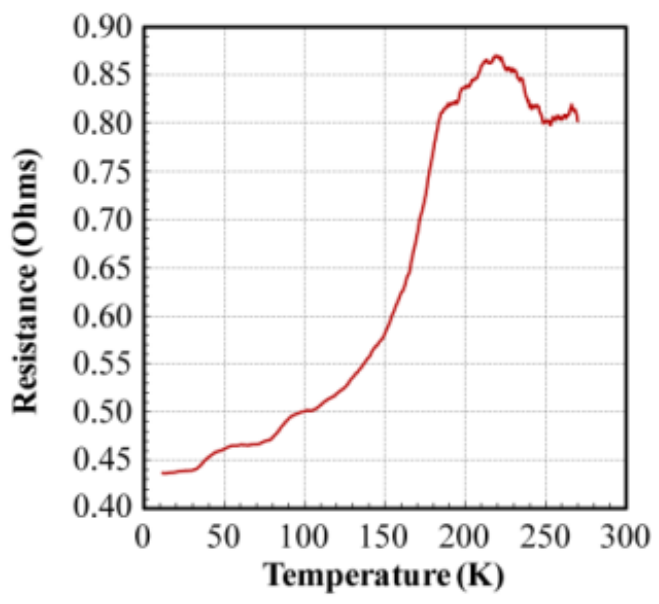

b

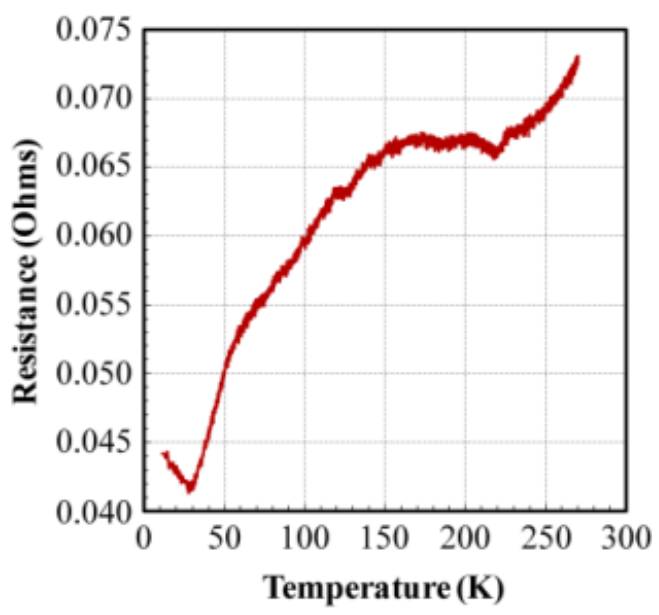

d

Figure 32: Measured $R$ vs. $T$ of four thin films exfoliated from phosphorousimplanted (Ep $=10 \mathrm{keV}$, dose $1.2 \times 108 \mathrm{~cm}-2)$ HOPG samples. (a) HOPG-008, layer 3; (b) HOPG-019, layer 3; (c) HOPG-019, layer 6; (d) HOPG-021, layer 7. The layer numbers indicate the number of multilayers peeled from the host sample, i.e. layer 7 would be the $7^{\text {th }}$ layer exfoliated from that sample.

To see if additional lattice damage by neutral ion species could increase pinning, which could only occur if magnetic vortices were present, and reduce losses, a sample which had been previously implanted with phosphorous but had not yet been exfoliated was sent back for implantation with argon. This implantation was done at reduced energy $(5 \mathrm{keV})$ and the same dose as the phosphorous implantation $\left(1.2 \times 10^{8} \mathrm{~cm}^{-2}\right)$ to place the 
damage in front of the peak in the phosphorous distribution. If the resistance was indeed due to flux-flow, a stronger pinning would be observed in the $R$ vs. $T$ characteristic.

Figure 33 shows the computed range distributions for the phosphorous (1) and the argon (3) implants in this HOPG sample and the computed damage distributions caused by implanted phosphorous (2) and argon (4).

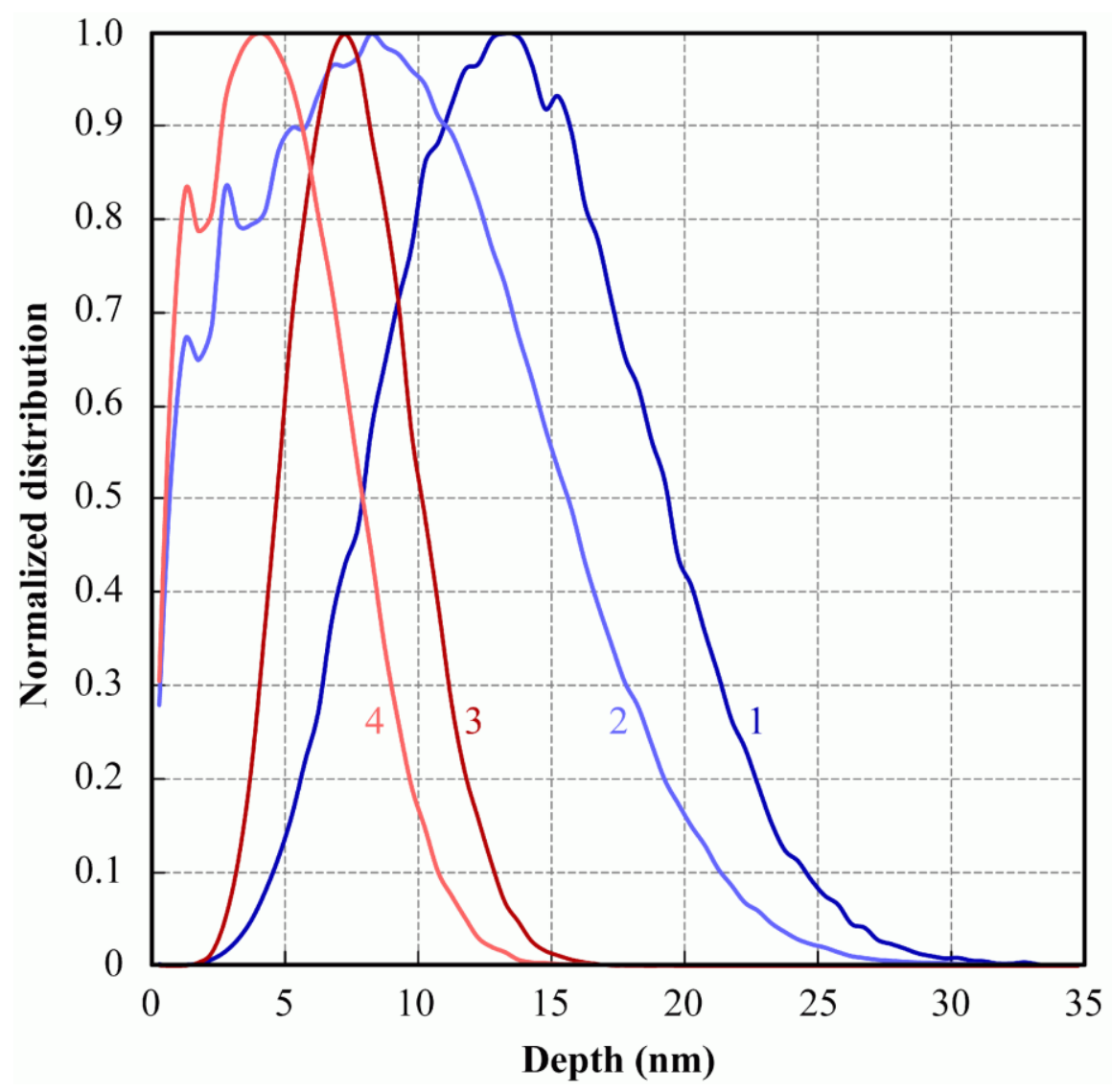

Figure 33: This represents the simulated normalized distributions of implanted atoms and lattice damage caused by implanted atoms versus depth in HOPG. This represents the simulated normalized distributions of implanted atoms and lattice damage caused by implanted atoms versus depth in HOPG. Curve 1 is the calculated distribution of the implanted phosphorous, curve 2 is the computed damage caused by the implanted phosphorous, curve 3 is the calculated distribution of the implanted argon and curve 4 is the computed damage caused by the implanted argon. 
The $R$ vs. $T$ characteristic of an exfoliated graphene multilayer from this doubly implanted sample (phosphorous followed by argon) is shown in curve 1 of Figure 34 . Note that the first $R$ vs. $T$ characteristic taken showed the same qualitative behaviour as the samples in Figure 32.

An anomaly in the form of a small notch can be observed in curve 1 of Figure 34 overleaf. Its size (18 data points each from 250 averaged measurements) is considered too great to be either noise or a measurement error. This notch is at a temperature of $132 \mathrm{~K}$ and gave cause to re-measure the sample multiple times. These re-measurements were performed without disturbing the sample or altering the refrigerated environment. In these subsequent $R$ vs. $T$ re-measurements, shown as curves 2 through 4 in Figure 34, a large and abrupt step was observed. The step was in the temperature region of 210-230 K on the first re-measurement. On a second re-measurement it was observed to have moved upward to a temperature of 250-260 K. On a third, and final, re-measurement, the step was noted to have migrated upwards to a temperature of $264-267 \mathrm{~K}$. 


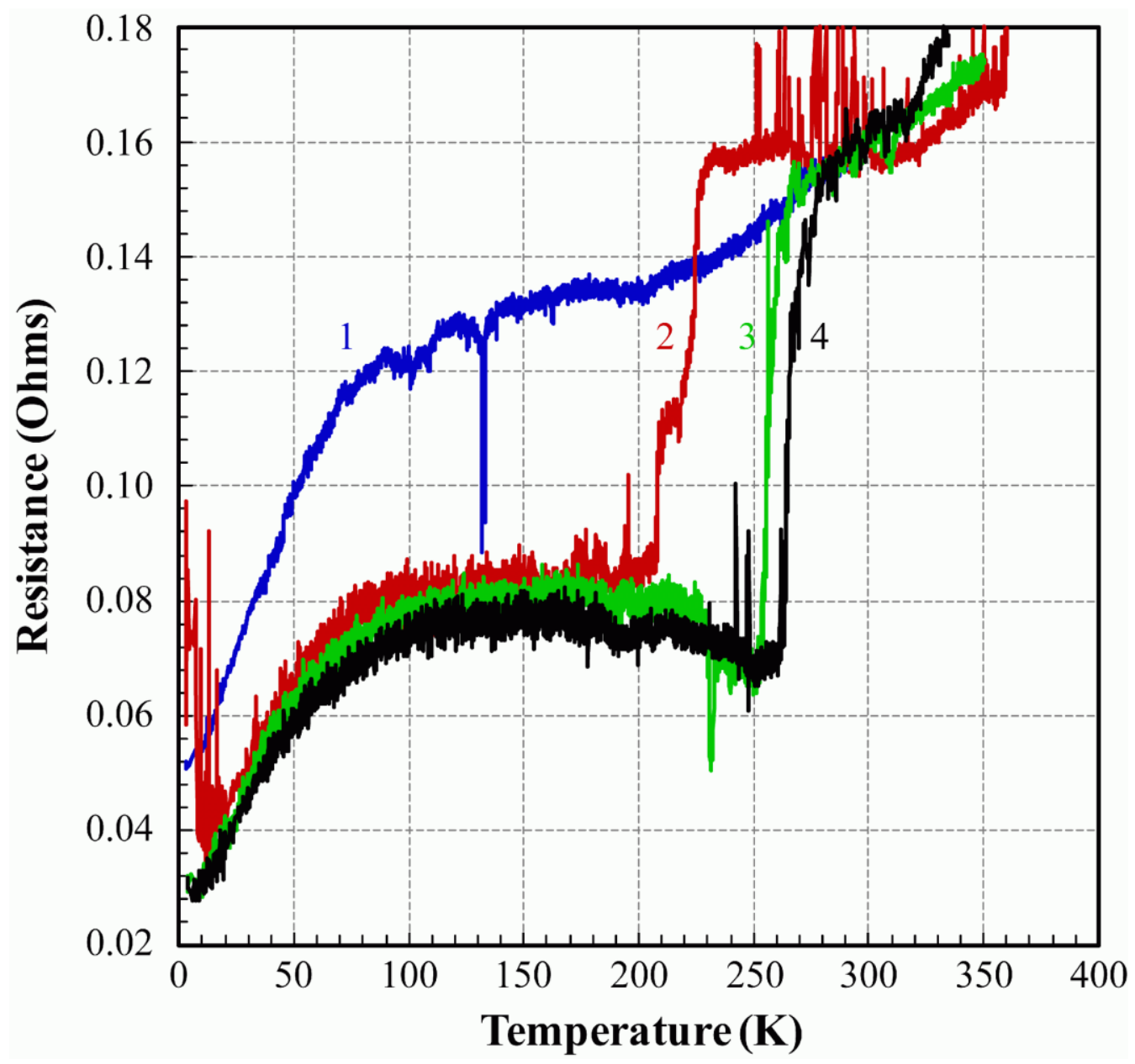

Figure 34: $R$ vs. $T$ of a thin film sample peeled off phosphorous-implanted and then argon-implanted bulk HOPG-023. Curves 1 to 4 are four identical sequential runs with the same probe position.

It can be determined that there is no contact issue present when all four $R$ vs. $T$ characteristics for the graphene multilayer from sample HOPG-023 are plotted together on the same graph (Figure 34). It can be seen that the low temperature and the high temperature resistances have not been changed significantly from run to run. It is also clear that the notch observed in the first $R$ vs. $T$ characteristic is an attempt for the material to move to the resistance state achieved in the later runs and cannot be dismissed as spurious. 


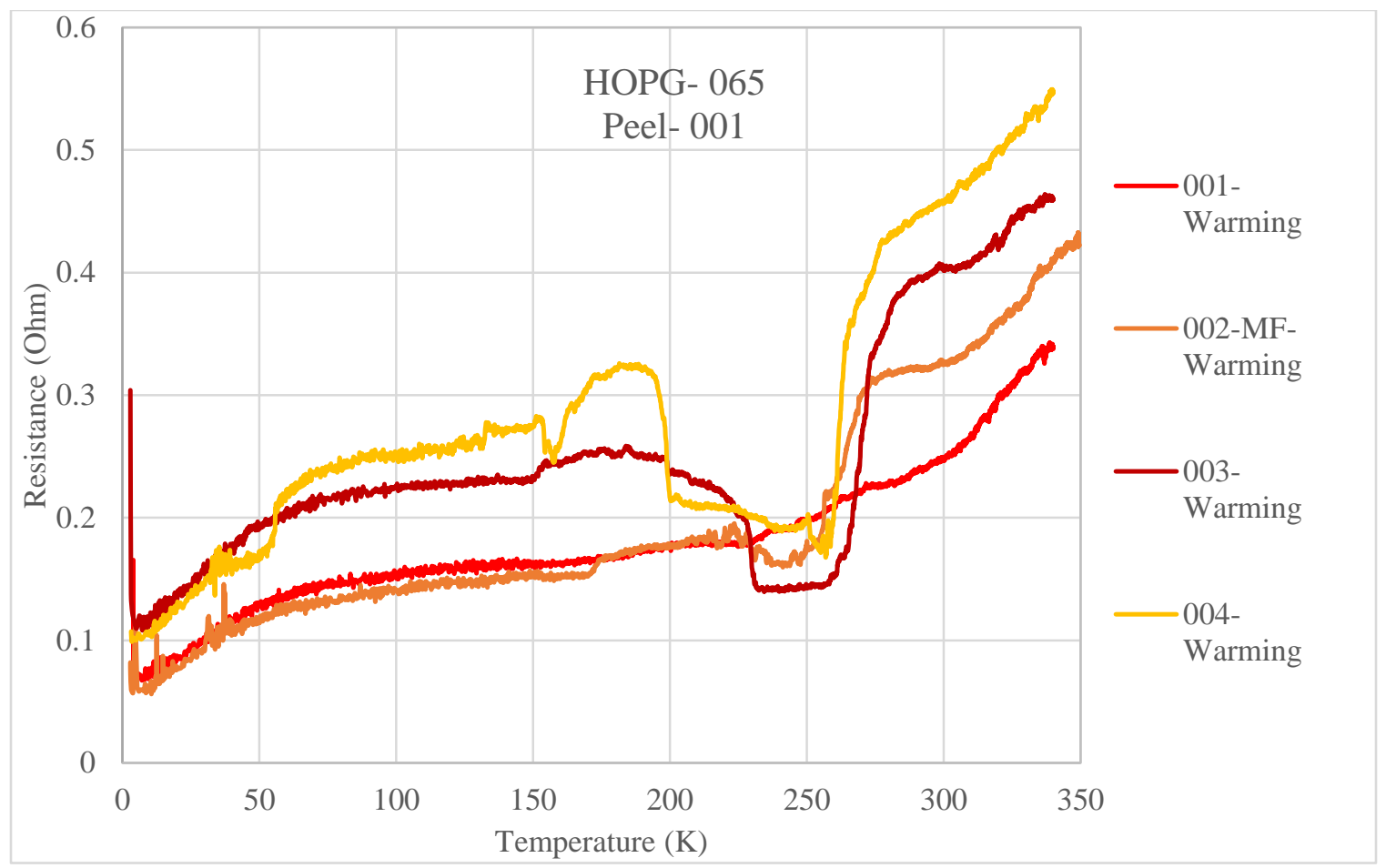

Figure 35. Multi energy implantation Sample 065.

For our multi energy ion-implantation sample seen in Figure 35, we see prominent valleys at around $260 \mathrm{~K}$ for all runs except the initial run. This valley first appears when the magnetic field is applied and continues to grow after the field is removed for the following runs. On the fourth run, this valley continues all the way down to $200 \mathrm{~K}$. This feature is similar to the phosphorus followed by argon multi energy implantation in Figure 34 . 


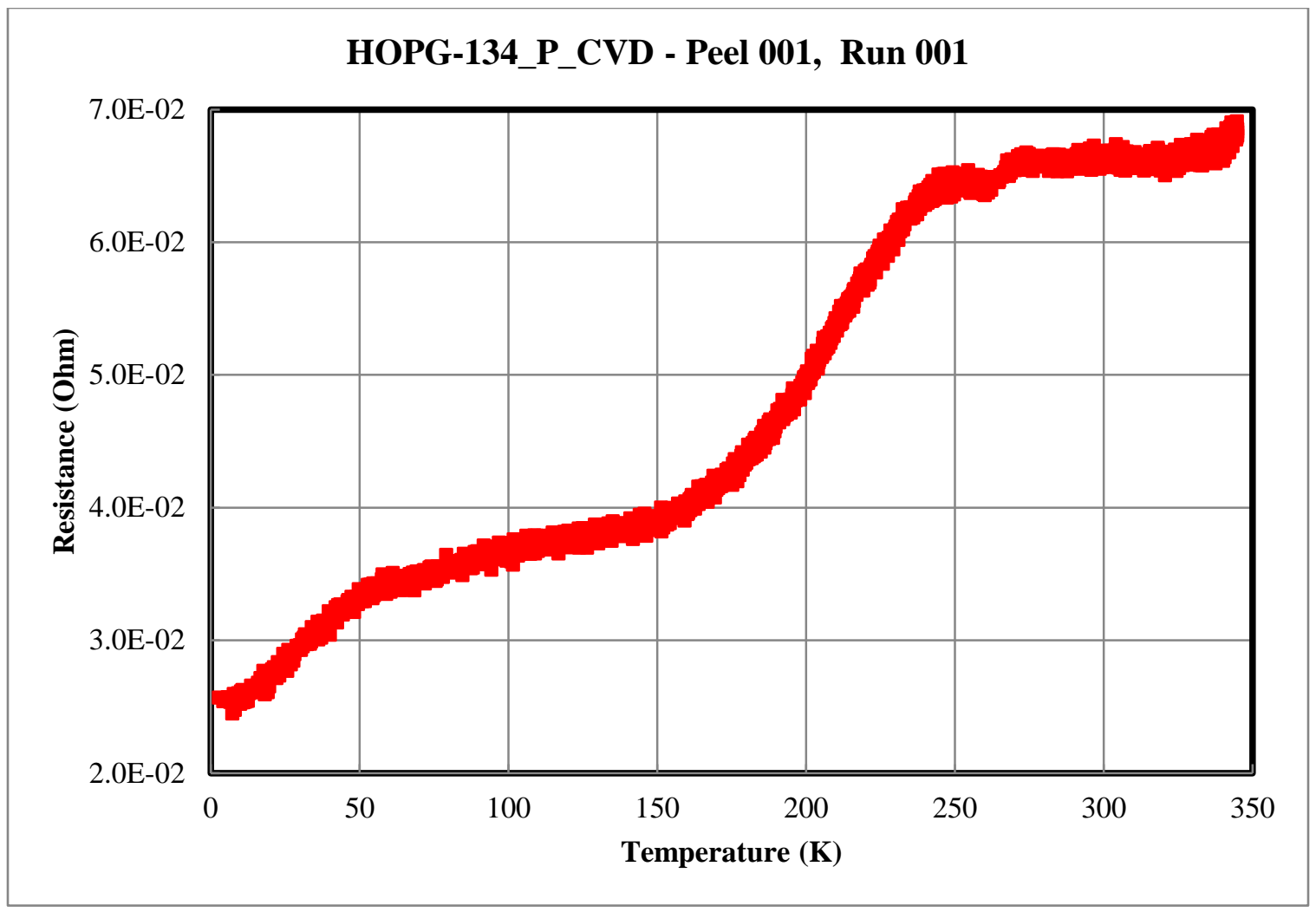

Figure 36. PECVD graphene grown in the presence of phosphine. First peel taken from bulk HOPG.

In Figure 36 we see a representative $R$ vs. $T$ measurement of a graphene grown in the presence of phosphine using our custom plasma enhanced CVD system. The transition can clearly be seen at $240 \mathrm{~K}$ and leveling off at $150 \mathrm{~K}$ before dropping again at $50 \mathrm{~K}$. As expected, since the flux vortices are not pinned, they are free to flow, rather than being abruptly dismantled as see in the ion-implanted samples. This transition has been seen in over 50 of our samples.

The SQUID magnetometer measurements of a doped-while-grown exfoliated thin film are shown in Figure 37. They consist of a Zero-Field Cooled magnetization run followed by a Field Cooled magnetization run. The hysteresis loop in the Zero-Field Cooled to Field Cooled curves begins to open at a temperature of approximately $260 \mathrm{~K}$. 


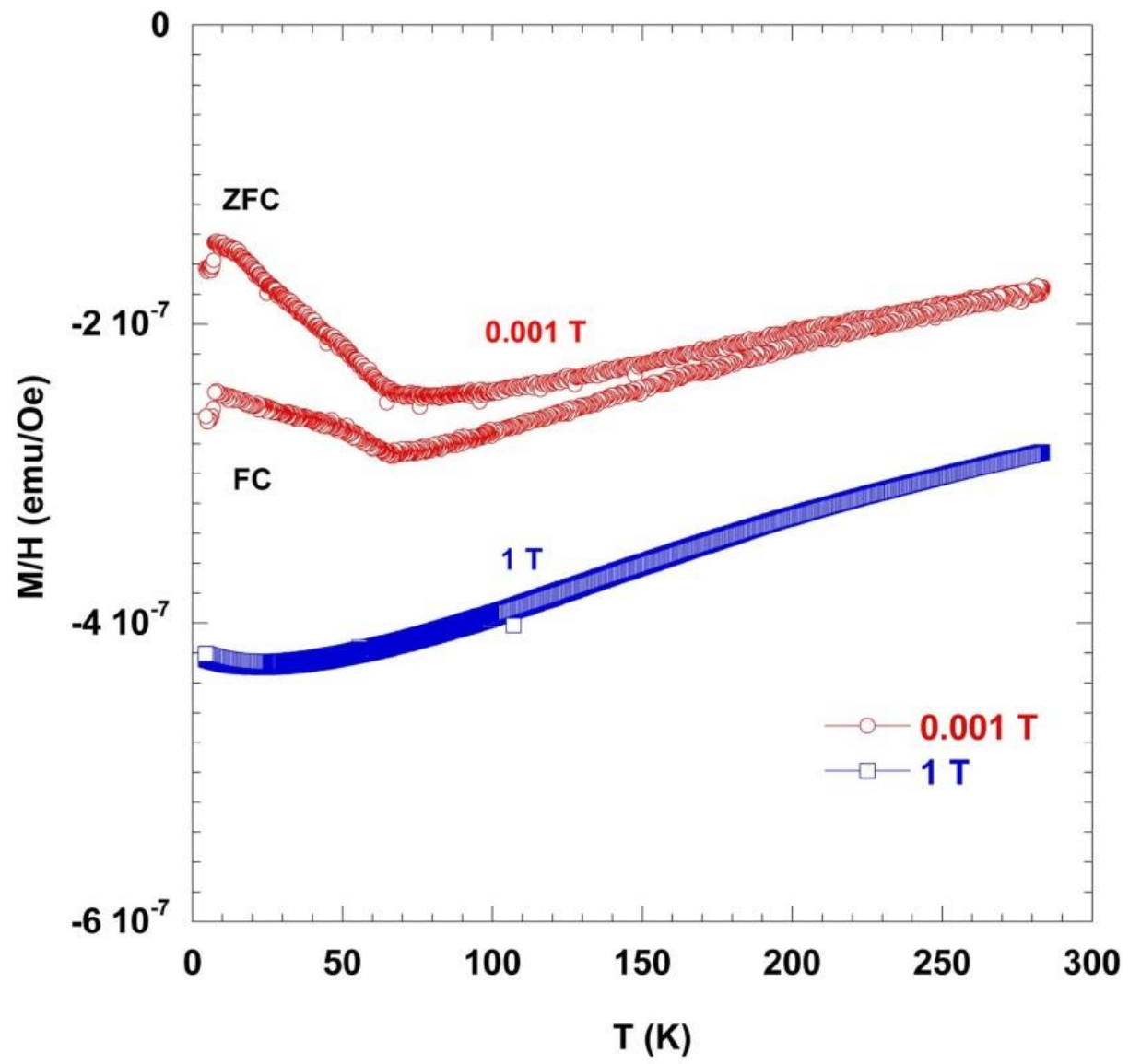

Figure 37: Magnetization measurements: Zero-Field Cooled (ZFC) and Field Cooled (FC) M/H plots along with a $1 \mathrm{~T}$ Field Cooled M/H plot for a film exfoliated from a doped-while-grown Plasma Chemical Vapor Deposition sample.

Additionally, when the horizontal axis of the Zero-Field Cooled magnetization run is converted from $\mathrm{T}$ to $\mathrm{H} / \mathrm{H}_{\mathrm{C} 2}$ (temperature to the Magnetic Field / Upper critical field of the vortex state) and the data is re-plotted, similar results are attained to those seen in the experiments and calculations done by Berdiyorov's [277] and Novosolev [278]-[287] The Figure 38 (Fig 3.2 in [277]) shows the qualitative shape of a pancake vortex array in a type I superconductor. The thickness here is much less than the magnetic penetration 
depth (ratio less than unity). Figure 38(d) would provide the greatest similarity to the samples used in this work as it has no "hard pinning" sites (anti-dots).

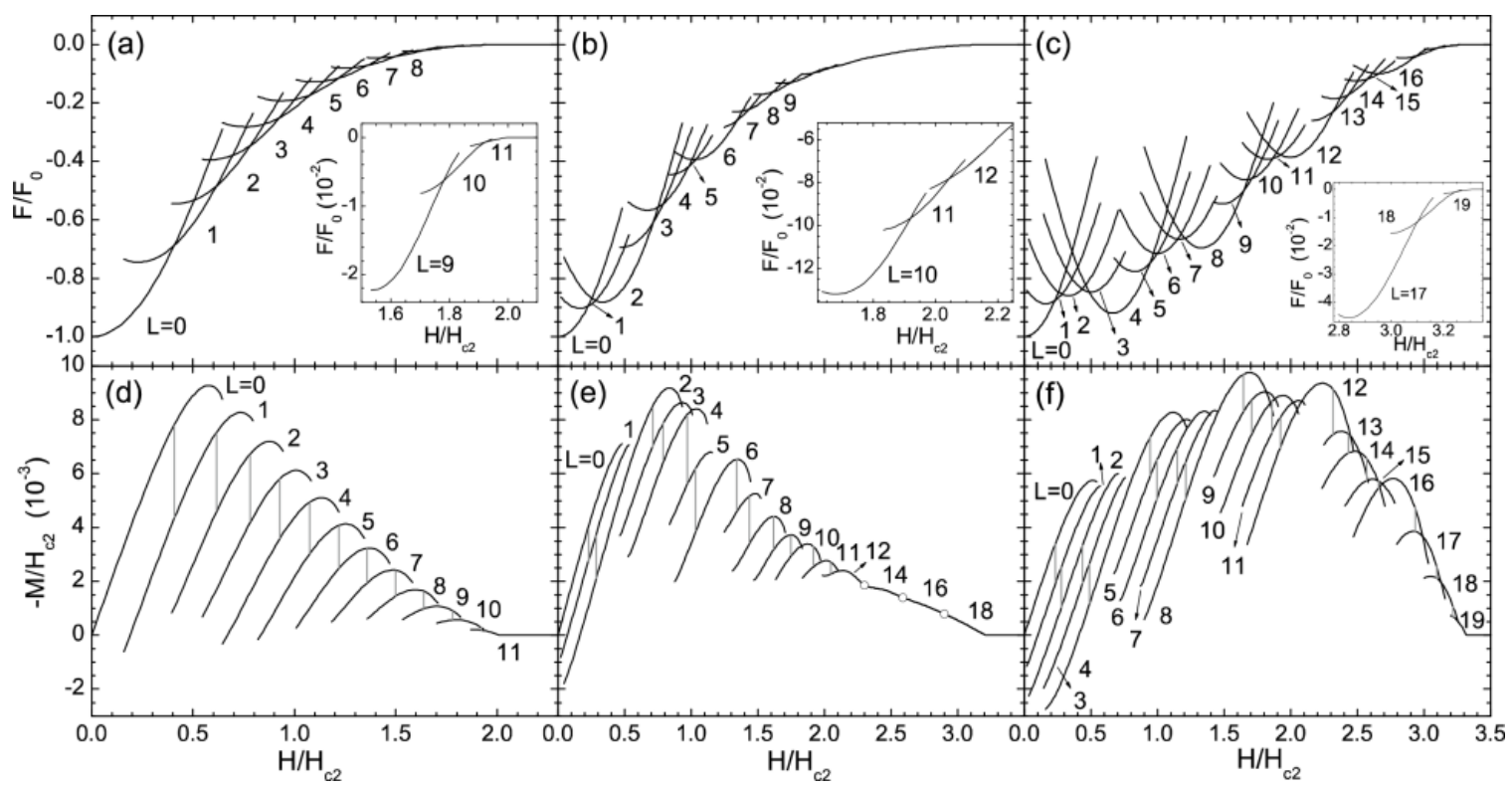

Figure 38: The free energy (a-c) and magnetization (d-f) as a function of the applied magnetic field for a filled square $(a, d)$ and for the square with two $(b, e)$ and four (c,f) anti-dots. The insets show the free energy for higher vorticity. The vertical lines show the ground state transitions between different vortex states and open circles indicate continuous transitions between different vortex states. The GL parameter is $k=0.28$ [277].

Re-plotting the data of the phosphorous-doped-while-grown Chemical Vapor Deposition exfoliated graphene film Zero-Field Cooled curve given in Figure 37, a comparison can be made with Figure 38(d). The temperature information was converted to the ratio of $\mathrm{H}$ to $\mathrm{H}_{\mathrm{C} 2}$ so as to make the comparison with Figure 38(d) [277]. The best comparison that can be made qualitatively is via observing the shape of the curves. This is due to the fact that there is no known value for $\mathrm{H}_{\mathrm{C} 2}$ for the phosphorous-doped-whilegrown Chemical Vapor Deposition exfoliated graphene film. This is shown in Figure 39. Note the step in the curve at low $\mathrm{H} / \mathrm{H}_{\mathrm{C} 2}$ in Figure 39. This corresponds qualitatively to 
one of the discontinuous jumps in Berdiyorov's data and, should the measurement temperature have been taken to lower values, would most likely have continued vertically.

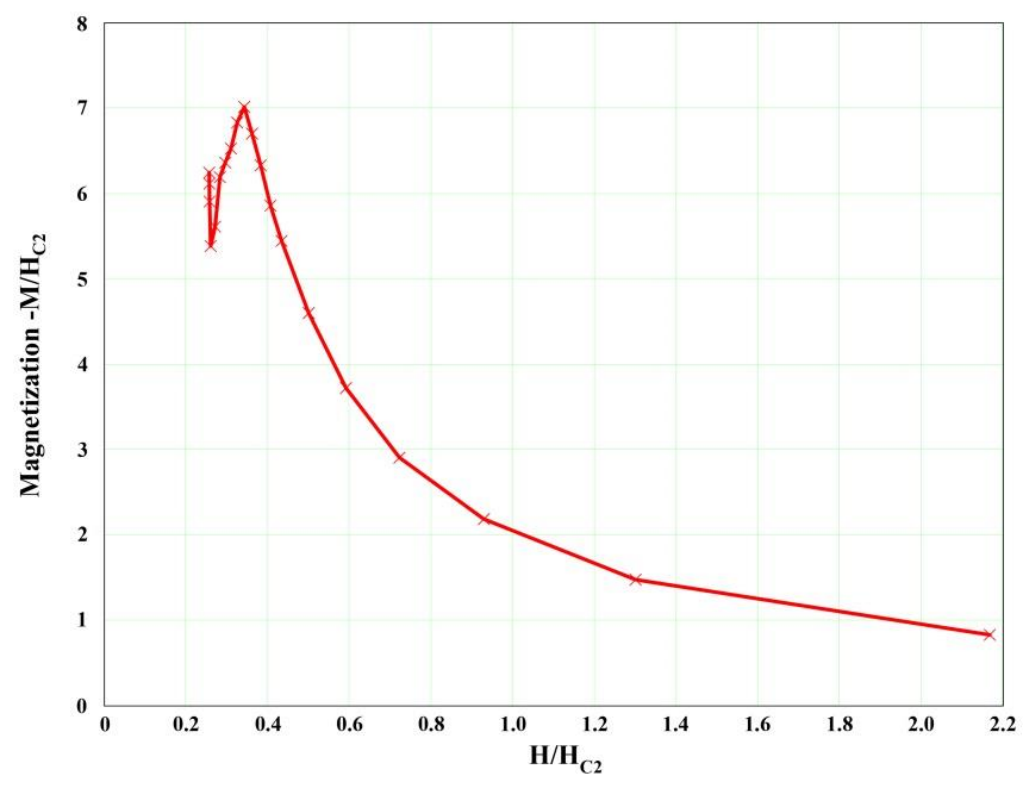

Figure 39: Phosphorous-doped during growth in plasma Chemical Vapor Deposition graphene sample zero-field-cooled magnetization results plotted on same axis type as used in Berdiyorov's work. Note same general shape as in Figure 38(d).

The AC susceptometer measurements for a representative doped-while-grown exfoliated thin film are shown in Figure 40(a) and Figure 40(b). In addition to each of the full-scale plots, a corresponding plot with a magnified vertical axis is shown. A representative plot from a known superconductor is also included as Figure 41 for comparison. Note the small transition that is relatively broad and begins in the area of $150 \mathrm{~K}$ (this may be depressed as a result of the measurement field). 


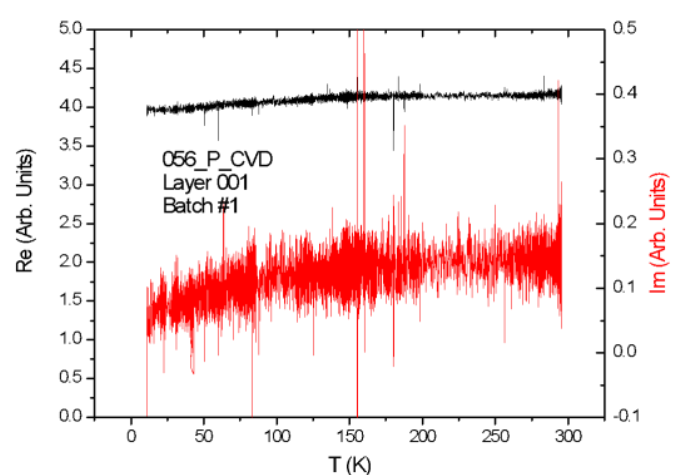

(a)

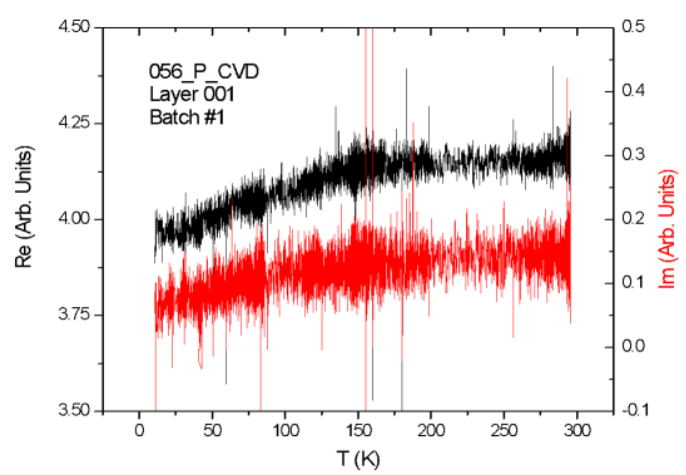

(b)

Figure 40: AC Susceptometer results for a graphene film on Kapton ${ }^{\circledR}$ tape exfoliated from a phosphorous-doped-while-grown Chemical Vapor Deposition sample. (a) film was cut into multiple pieces and the pieces were stacked to increase the screening fraction; (b) magnified vertical axis AC susceptometer results for the same sample and run as shown in (a).

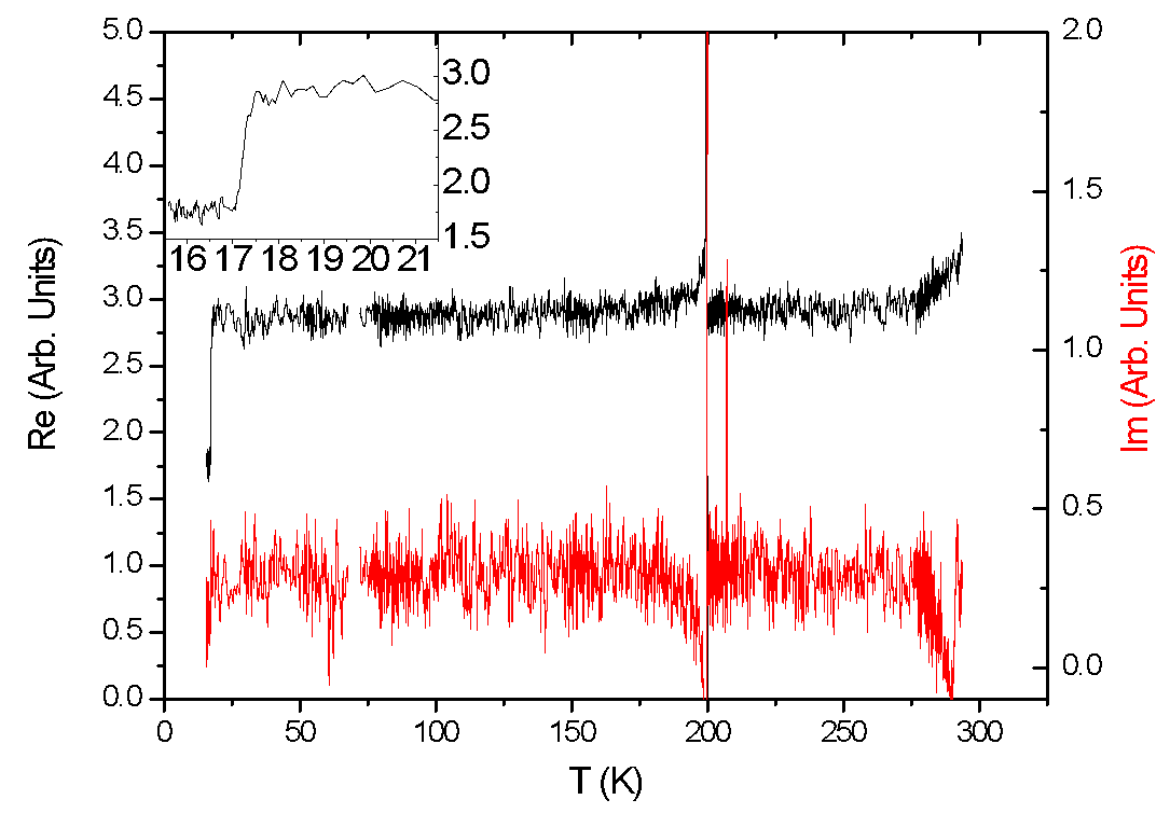

Figure 41: AC Susceptometer results for a $\mathrm{Pr} 2-\mathrm{xCexCuO4}$ superconductor in the same instrument as was used to produce the data shown in Figure 40. The magnified transition region is shown in the inset. 
The final measurements that have been done included a Raman spectrum of one of our exfoliated films to estimate the film's thickness (Figure 42).

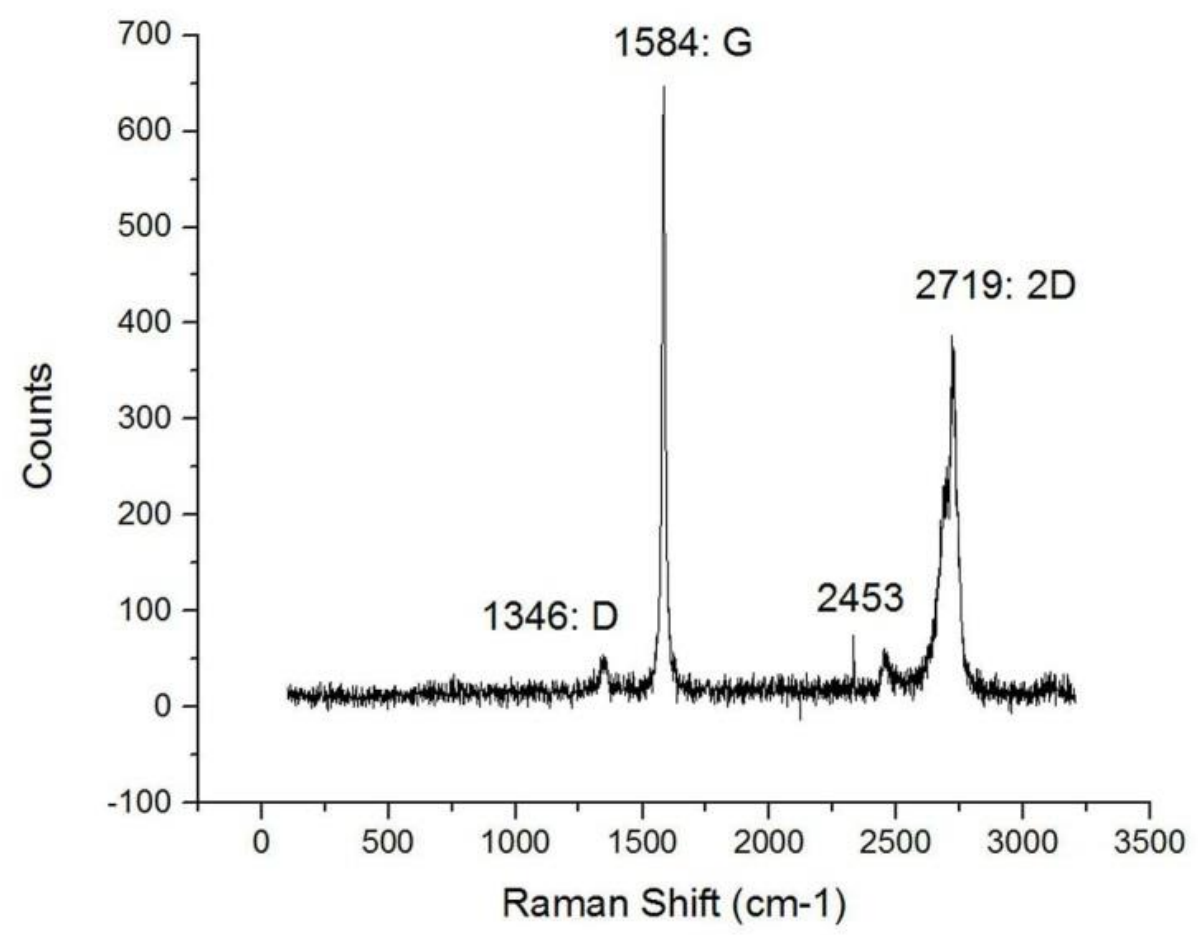

Figure 42: Raman spectra for a film on Kapton ${ }^{\circledR}$ tape exfoliated from a phosphorous-doped-while-grown Chemical Vapor Deposition graphene sample. The peak ratios give a thickness of approximately 5 monoatomic layers.

The number of layers can be determined by analysing the ratio G/2D. The Raman spectrum plot in Figure 42 indicates a G/2D ratio of $646 \mathrm{~K} / 385 \mathrm{~K}$, which is a little less than 2. This clearly indicates that the graphene material is multi-layered. As the G/2D ratio for 5 monolayers of graphene is about 3.8, the measurements suggest that the exfoliated layers are composed of about 5 to 6 monolayers. 
Hall Effect measurements at temperatures of $297 \mathrm{~K}$ and $80 \mathrm{~K}$ are shown in Figure 43. The change in sign of the Hall voltage is not due to issues in instrumentation. The contacts and extraction calculations were left unaltered between both runs.

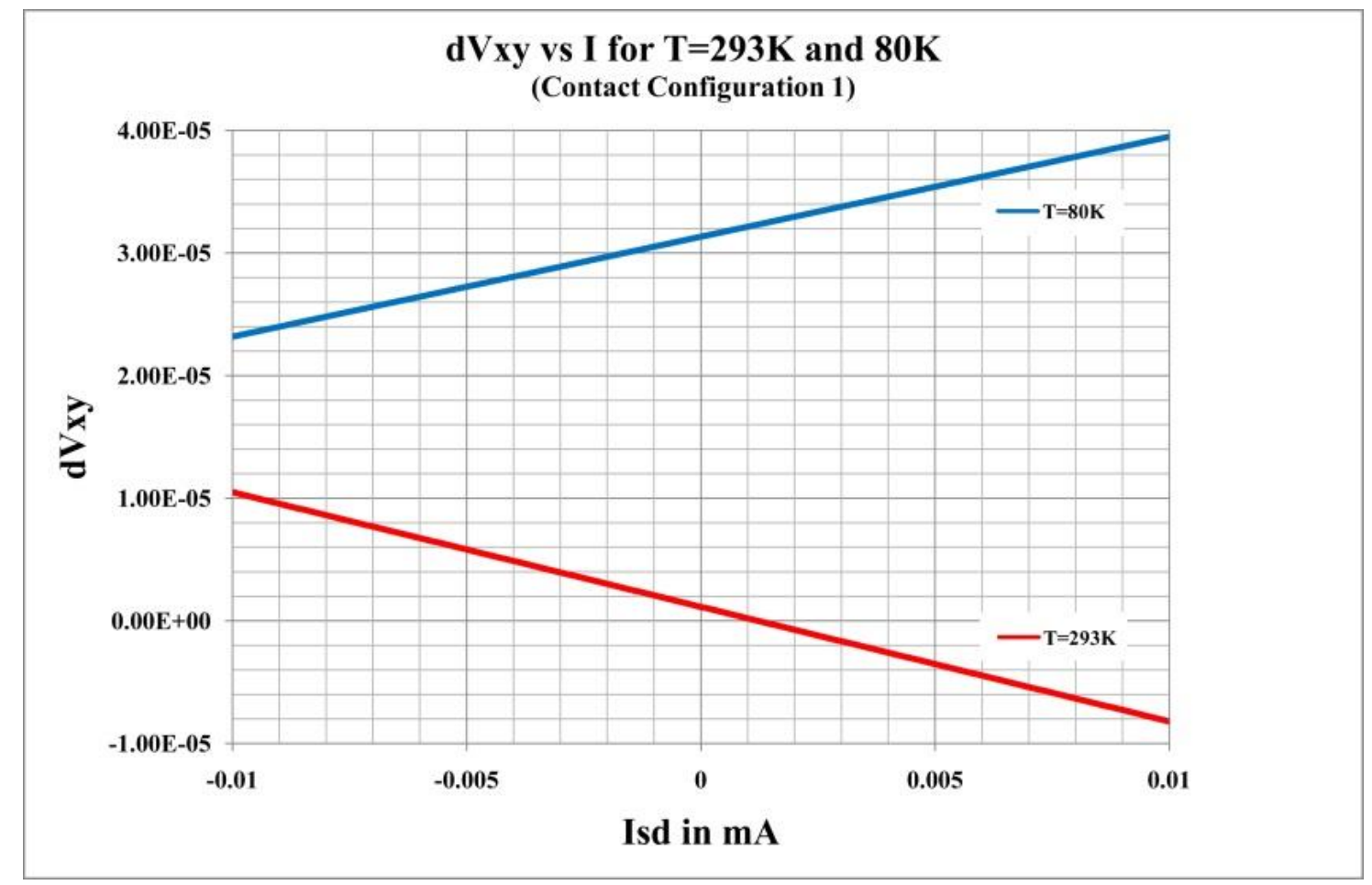

Figure 43: Hall effect at $T=293 \mathrm{~K}$ and $\mathrm{T}=80 \mathrm{~K}$ for the same film on Kapton ${ }^{\circledR}$ tape exfoliated from a phosphorous doped-while-grown Chemical Vapor Deposition graphene sample as is shown in the Raman spectrum in Figure 42.

For both positive and negative field directions, four different configurations were averaged in resistivity mode and Hall configurations via use of a Hall card and switch from a Keithley model 7001 Switch System.

The great amount of coupling in the conductivity tensor $\left(\mathrm{V}_{\mathrm{xx}}\right.$ and $\left.\mathrm{V}_{\mathrm{xy}}\right)$ required the data to be symmetrized in order to yield only the induced magnetic field. This is shown in Figure 44. (i.e. $\left[\mathrm{V}_{\mathrm{xy}_{-}+\mathrm{B}}-\mathrm{V}_{\mathrm{xy}_{\_} \mathrm{B}}\right]$ ) 


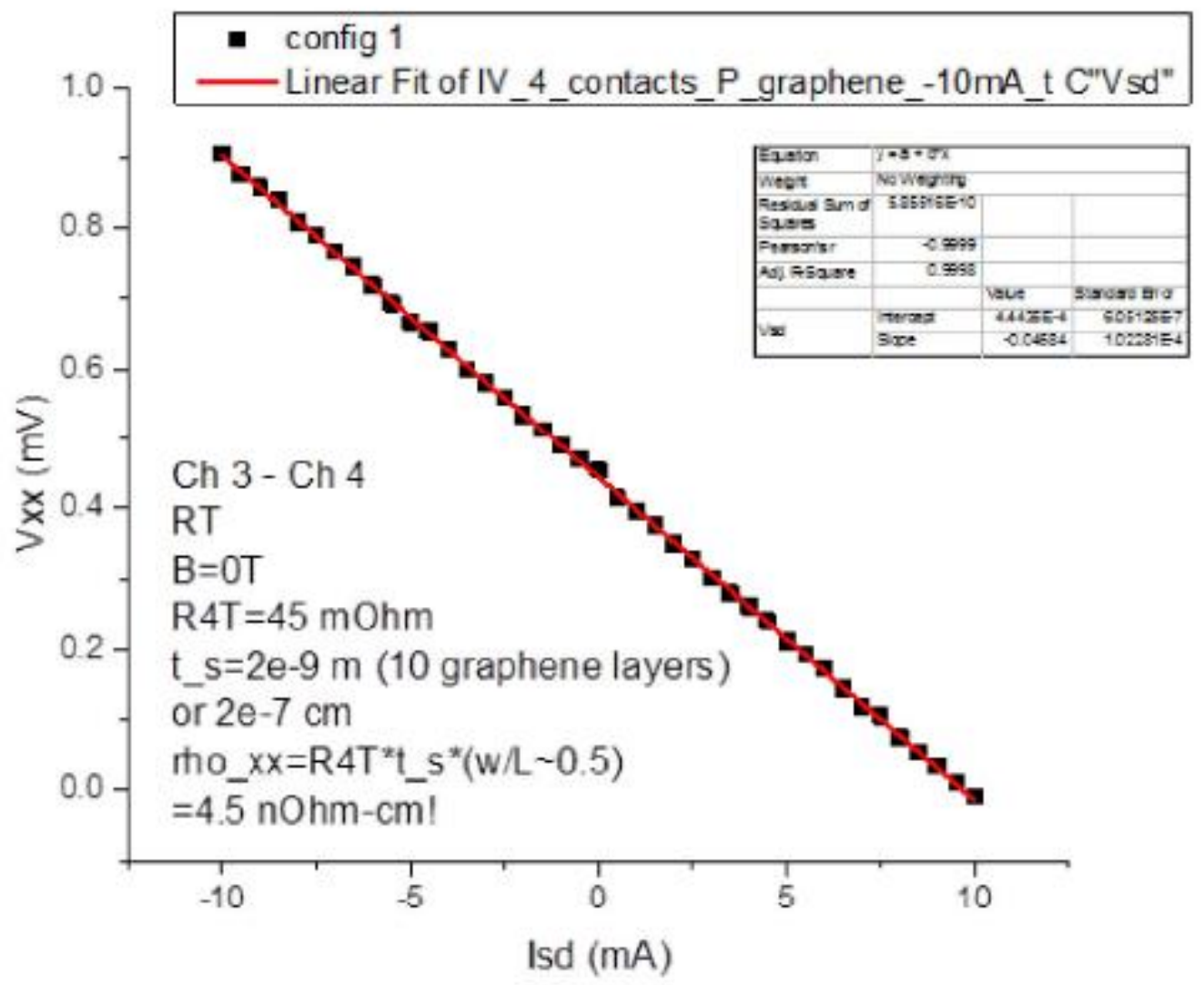

Figure 44: Graph of the four point resistance (averaged over all contact configurations) of the device at room temperature and in $0 \mathrm{~T}$ field. Vxx preamplifier reversed the polarity. 4 terminal resistance of the film determined to be $45 \mathrm{mOhm}$.

The Hall signal shown below in Figure 45 (red trace in Figure 43) suggests a two dimensional carrier density of $4.70 \times 10^{17} / \mathrm{cm}^{2}$. This result was seen after the different voltage probe and current configurations were made symmetric at room temperature. Using the resistivity obtained from the data shown in Figure 44, an effective mobility of $590 \mathrm{~cm}^{2} / \mathrm{Vs}$ was calculated. This result is dependent on knowing the exact sample thickness which is somewhat uncertain due to roughness and topological factors. 


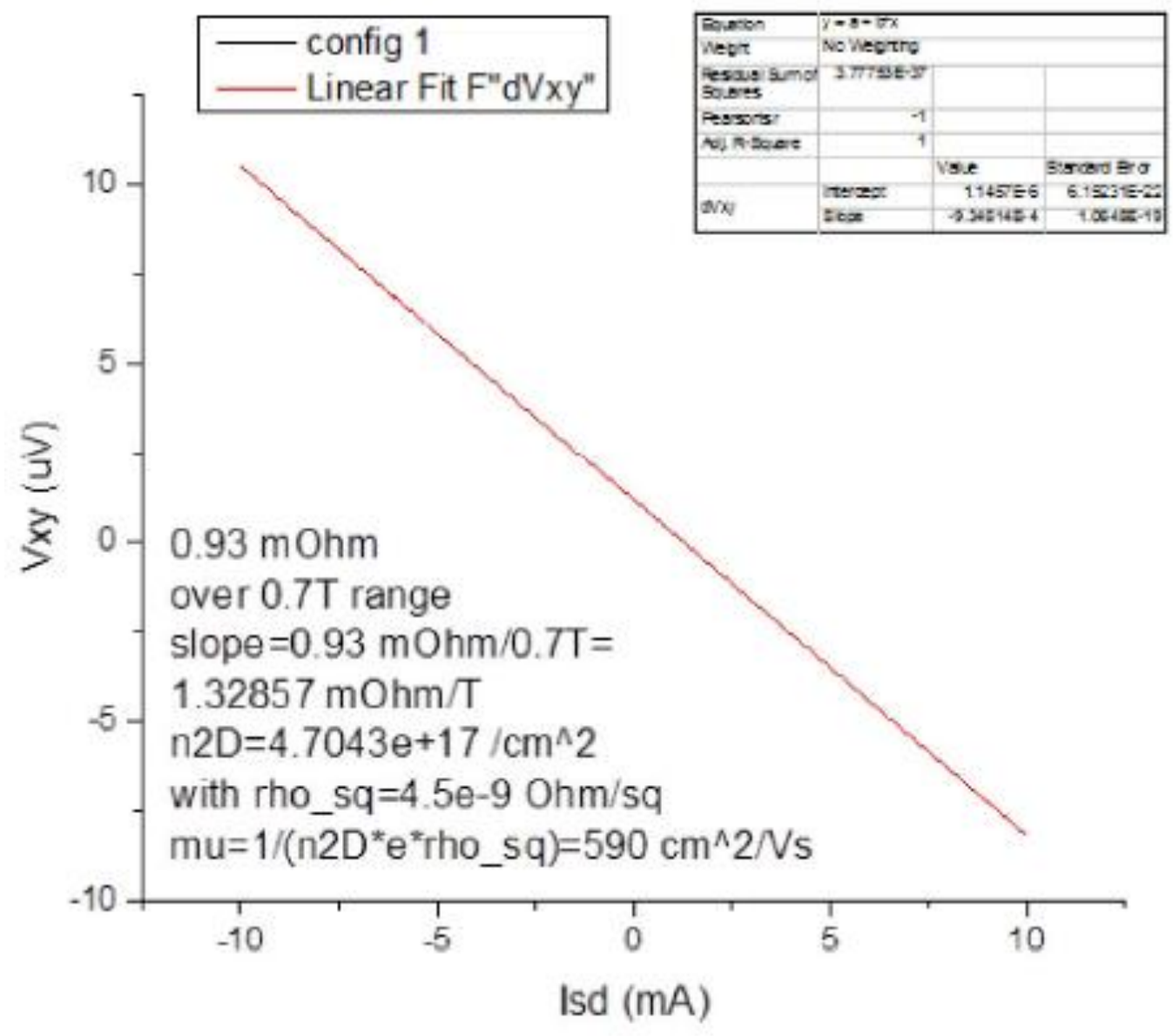

Figure 45: Graph of the Hall signal after symmetrization the different voltage probe and current configurations were made symmetric at room temperature.

Figure 46 (blue trace in Figure 43) shows the graph of the Hall voltage at $80 \mathrm{~K}$ taken after symmetrisation. As can be seen, the density (Hall slope) has not changed dramatically, but increased slightly. 


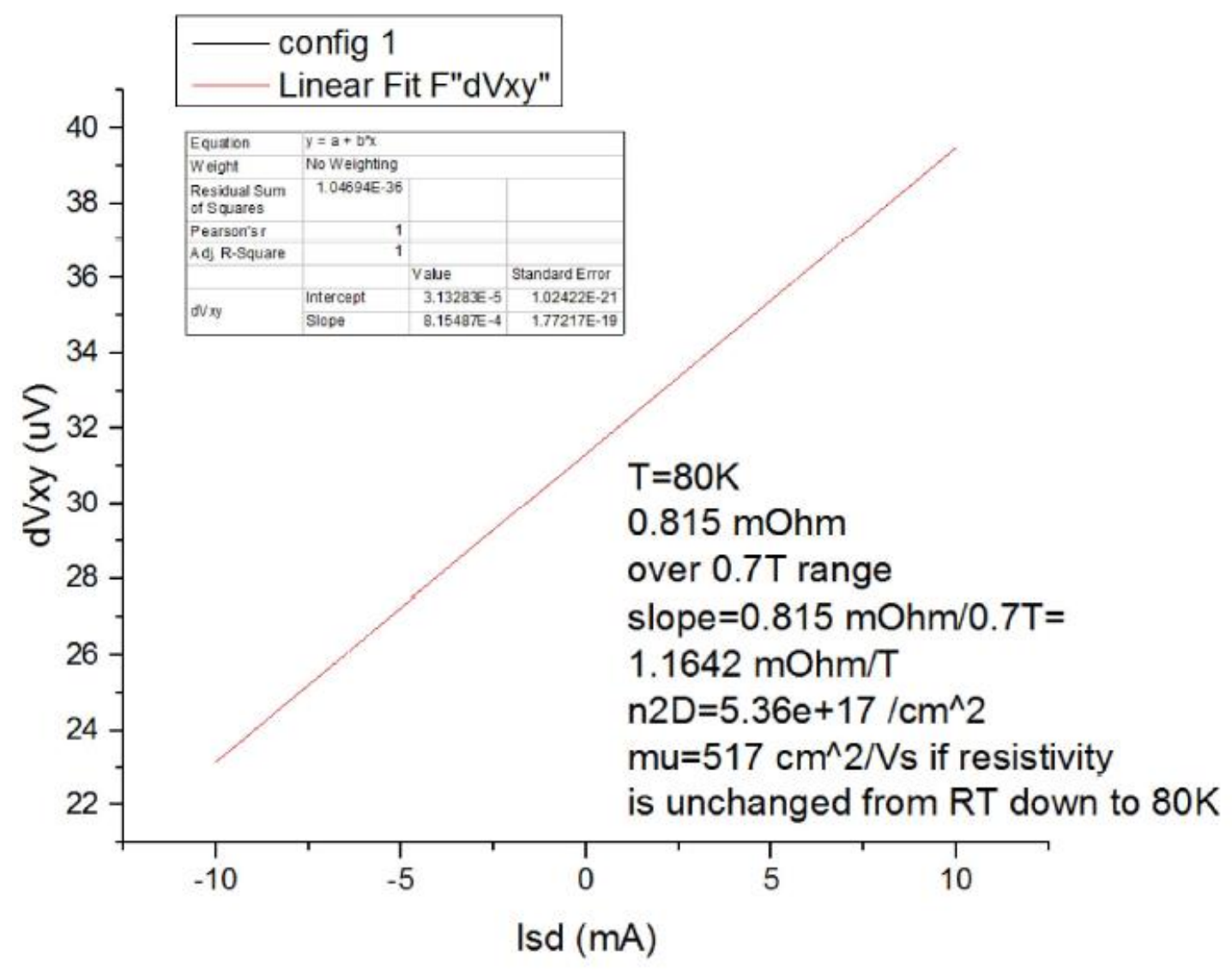

Figure 46: Graph of the Hall signal after symmetrization the different voltage probe and current configurations were made symmetric at $80 \mathrm{~K}$.

\section{DISCUSSION}

To verify that our delta measurement cryogenic system was working correctly, we did several $R$ vs. $T$ measurements using known superconductors. Since we know what the output should look like for the $R$ vs. $T$ measurement, it would be easy to deduce what problems we were having with our system, if any. The first superconductor that we tested was a $\mathrm{YBa}_{2} \mathrm{Cu}_{3} \mathrm{O}_{7-\mathrm{x}}$ target that was grown in-house, several years prior. The thickness of these targets was $3 \mathrm{~mm}$ while being $8 \mathrm{~mm}$ in diameter. Since the volume 
was large, we expected to see a clear response. A current bias of $1 \mathrm{~mA}$ was used after trying several biases out to see which gave the cleanest signal while still giving us a high level of sensitivity. Since the delta resolution decreases a decade for every decade increase in current bias, keeping the current bias low was the key. Lanthanum Aluminate was placed under the $\mathrm{YBa}_{2} \mathrm{Cu}_{3} \mathrm{O}_{7-\mathrm{X}}$ target to prevent shunting of superconductivity while also being thermally conductive. Our collinear probe setup was then positioned on the sample, using the same positioning and spacing that we would be using for our HOPG samples. Due to $\mathrm{YBa}_{2} \mathrm{Cu}_{3} \mathrm{O}_{7-\mathrm{x}}$ 's rough surface, Indium dots were placed between the probes and $\mathrm{YBa}_{2} \mathrm{Cu}_{3} \mathrm{O}_{7-\mathrm{x}}$ surface. Since Indium is an extremely malleable material it was able to enhance contact when pushed into the surface of the $\mathrm{YBa}_{2} \mathrm{Cu}_{3} \mathrm{O}_{7-\mathrm{x}}$. Because the probe spacing is so much greater than the coherence length of $\mathrm{YBa}_{2} \mathrm{Cu}_{3} \mathrm{O}_{7-\mathrm{X}}$, the addition of Indium would not affect the superconductive behavior [137]-[139]. On cooling we see that it starts transitioning at 21 Kelvin, then reaching zero resistance at 10.6 Kelvin (see $\mathrm{R}$ vs. T for YBa2Cu3O7-X Target in Appendix 1 ). On warming, it starts transitioning at 88 Kelvin, and reaches zero resistance at 68 Kelvin. This is exactly what we expect to see from a sample of this thickness. The difference in the transition temperature for cooling and warming are largely due to the volume of the sample. The top surface of the target will take longer to cool than the thermocouple under the stage, where the temperature readings are taken. These are the transition temperatures we expected, so we know that our temperature measurements as well as cooling system are working correctly. There's also a lack of noise in this measurement, which is ideal. These are the transition temperatures we expected, so we know that our temperature measurements as well as cooling system are working correctly. 
The $\mathrm{YBa}_{2} \mathrm{Cu}_{3} \mathrm{O}_{7-\mathrm{x}}$ Hairpin filter is 1 millimeter thick and 8 millimeters square. Lanthanum aluminate was placed under the sample in the same manner as the $\mathrm{YBa}_{2} \mathrm{Cu}_{3} \mathrm{O}_{7}$. $\mathrm{x}$ target aforementioned, as well as Indium dots being placed under the probes to create better contact. The Hairpin filter has a higher density than the target, with a smoother surface. For the $R$ vs. $T$ measurement, we see that the Hairpin sample experiences a transition on cooling of 42 Kelvin (see R vs. T for YBa2Cu3O7-X Hairpin in Appendix 2 ). It reaches zero resistance at 27 Kelvin. For Warming, it begins the transition at 92 Kelvin, and reaches zero resistance at 83 Kelvin. The transition temperature between warming and cooling has a difference of 50 Kelvin whereas for $\mathrm{YBa}_{2} \mathrm{Cu}_{3} \mathrm{O}_{7-\mathrm{x}}$ Target, the difference was 67 Kelvin. This makes sense because as a sample gets thinner, the transition temperature will be closer for Warming and Cooling since the sample is cooling at a faster rate on the surface. The current bias for the Hairpin filter was 1 micro amp. Since the current was far lower for this measurement, we see a result that is noisier than the Target, which used 1000 times more current.

To further verify our system, we performed an $R$ vs. $T$ measurement using Niobium which is an element type II superconductor (see R vs. T for Niobium in Appendix 3 ). We used the same set of procedures as the $\mathrm{YBa}_{2} \mathrm{Cu}_{3} \mathrm{O}_{7-\mathrm{X}}$ Target and Hairpin samples, with the exception of using the Indium between the probes. This was not needed due to the smoother surface of the Niobium. A bias of 1mA was used during cooling but had slightly more noise than desired, so the bias was raised to $10 \mathrm{~mA}$ for warming. We see that the transition temperature is 9.244 Kelvin. We see an abrupt transition with only 1 data point between the initial transitioning temperature to its zero resistance state. Moreover, this in-between point is most likely due to the transitioning taking place 
during the 11 second measuring cycle and being averaged with the resistance before the transition period. Since Niobium has a transition temperature of 9.3 Kelvin, our measurement is only 0.056 Kelvin off. It is also worth noting that the warming run ended at 200 Kelvin due to a power failure. This was far beyond the transition temperature, so the run was not repeated. However, this inspired us to buy a large universal power supply to prevent this from happening in future runs.

During an $R$ vs. $T$ run on a bulk sample, we accidentally discovered what a loss of contact of our probes would look like during a measurement (see Loss of Contact in Appendix 4 ). The probes were placed on the sample with little to no pressure applied. This meant that there was very little contact being made to the surface of the HOPG at room temperature. During the cooling cycle, thermal contraction would cause the HOPG and lanthanum aluminate to get slightly smaller, resulting in a loss of contact mid run. This occurred at 146 Kelvin and then happened again on warming at 223 Kelvin. Since this is the bulk HOPG sample, it's expected to have a shift between warming and cooling since the top of the sample will not cool as fast, but we see drop to zero resistance instantaneously. This is indicative of a type 1 superconductor, and we know that if HOPG was to superconductive, it would be a type 2 superconductor. There was no noticeable change that we could see, caused by applying a magnetic field. During the cooling run, we watched it the entire time, and noticed that the indicator light at the bottom right-hand corner of the Keithley Model 6221 current source started to blink when it fell to the 0 resistance reading. According to the Keithley Model 6221 manual, this indicates that there is an open circuit. We performed a continuity test via the Triaxial connection on the chamber which connects to the current probes inside to verify this. 
Although this was accidental, this gave us a valuable look at what it is like to have a loss of contact during a run. Extra care was given in future runs to make ensure that firm pressure was applied to the probes for ample contact to the sample surface.

Arsenic is stripped of its 3 outermost electrons in the $\mathrm{S}$ and $\mathrm{P}$ orbitals, prior to ion implantation, to give it a crystal ionic radius of $72 \mathrm{pm}$. On impact with the HOPG surface, the charge neutralization process returns the electrons, giving it an atomic radius of $114 \mathrm{pm}$. At this radius, with an energy of $10 \mathrm{keV}$, the projected range is $104 \AA$ with a longitudinal straggle of $25 \AA$ and lateral straggle of $20 \AA$. When this pentavalent atom comes to rest, it becomes an electron donor for neighboring carbon atoms since it has 1 extra valence electron.

For the first run on the arsenic peel 001 which had a low dose implantation which yielded a concentration of $1.92 \mathrm{E}+14 / \mathrm{cm}^{3}$, there is an abrupt change in slope at 255 Kelvin, which continues to 148.77 Kelvin and levels off until reaching 94 Kelvin (see As Low Peel in Appendix 5. Directly after this run, and without taking the sample out of the chamber or disturbing the probes, we did another run using a magnetic field.

For the magnetic field run, we see that the slope between 94 Kelvin and 255 Kelvin is not as prominent. This is very subtle, and it is not clear if this is experiencing a transition due to flux flow.

For the high dose implantation which yielded a concentration in HOPG of $1.92 \mathrm{E}+17 / \mathrm{cm}^{3}$, we see a negative temperature coefficient down to 134 Kelvin (see As Hi Peel in Appendix 6 ). This phenomenon is seen in semiconductors and means that as the temperature increases, the number of active charge carriers increases. However, since we do not see a freeze out region, this would not be considered an intrinsic or even extrinsic 
semiconductor. There does not seem to be any sign of superconductivity at this concentration.

Aluminum is stripped of its 3 outermost electrons in the $\mathrm{S}$ and $\mathrm{P}$ orbitals, prior to ion implantation, to give it a crystal ionic radius of $67.5 \mathrm{pm}$. On impact with the HOPG surface, the charge neutralization process returns the electrons, giving it an atomic radius of $118 \mathrm{pm}$. At this radius, with an energy of $10 \mathrm{keV}$, the projected range is $149 \AA$ with a longitudinal straggle of $49 \AA$ and lateral straggle of $37 \AA$. When this trivalent atom comes to rest, it becomes an electron acceptor for neighboring carbon atoms since it has 1 less valence electron.

For Run 002 of our low dose implantation which had a concentration in HOPG of $9.8 \mathrm{E}+13 / \mathrm{cm}^{3}$, we see a decrease in resistance from 264 Kelvin down to 210 Kelvin (see Al Low Bulk Run 002 and Run 003 - MF in Appendix 7 ). This step appears to be a transition indicative of a type II superconductor. The resistance flattens until reaching 114 Kelvin where resistance descends again until reaching the bottom temperature. When a magnetic field was applied, the transition was suppressed by 14 Kelvin for the first transition temperature (250 Kelvin) and by 9 kelvin for the end of that transition (201 Kelvin). After that, the resistance flattens out to 114 Kelvin, which is identical to the previous temperature. When the magnetic field was removed, the first transition is identical to the magnetic field run, beginning at 250 Kelvin and ending at 201 Kelvin (see Al Low Bulk Run 003-MF and Run 004 in Appendix 8 ). The resistance flattens out for a longer range of temperatures, decreasing after 68 Kelvin.

We see a slightly negative temperature coefficient with the resistance remaining constant from 230 Kelvin to 183 Kelvin in our high dose Aluminum implantation with a 
concentration of $9.8 \mathrm{E}+16 / \mathrm{cm}^{3}$ (see Al High in Appendix 9 ). This resembles the 49 Kelvin window for the first transition seen in the low concentration sample, but shifted down 20 Kelvin. This shows that the lower concentration of aluminum ion implantation promoted features consistent with type II superconductors better than the higher concentration.

Nitrogen is stripped of its 3 outermost electrons in the $\mathrm{S}$ and $\mathrm{P}$ orbitals, prior to ion implantation, to give it a crystal ionic radius of $30 \mathrm{pm}$. On impact with the HOPG surface, the charge neutralization process returns the electrons, giving it an atomic radius of $56 \mathrm{pm}$. At this radius, with an energy of $10 \mathrm{keV}$, the projected range is $230 \AA$ with a longitudinal straggle of $84 \AA$ and lateral straggle of $62 \AA$. When this pentavalent atom comes to rest, it becomes an electron donor for neighboring carbon atoms since it has 1 extra valence electron.

For our low dose implantation with a concentration in HOPG of $5.71 \mathrm{E}+13 / \mathrm{cm}^{3}$, we see a transition at 283 Kelvin that ends at 261 Kelvin (see N Low, Peel 000, Run 002 and Run 003 - MF in Appendix 10 ). When the magnetic field was applied, although the transition ended at 261 Kelvin as well, we see it diverge from the previous pattern at 278 Kelvin. Instead of having a rounded apex, there appears to be a small amount of suppression present. Afterwards, the two trend lines converge. When the magnetic field run was followed by a non-magnetic field run, we see that the trend lines are very similar, and in some places, overlapping (see N Low, Peel 000, Run 003 - MF and Run 004 in Appendix 11. This may be due to residual flux vortices that were induced by the magnetic field still being present in the sample. This prompted us to remove graphene peels to explore the two-dimensional effect that the nitrogen low concentration doping 
may have been having. On the third exfoliation, we see that the first abrupt resistance decrease occurs at 329 Kelvin and ends at 319 Kelvin (see N Low, Peel 003, Run 002 and Run 003 - MF in Appendix 12 ). From 319 Kelvin to 280 Kelvin, there is a linear decrease in resistance. At 280 Kelvin, there is a linear increase in resistance to 235 Kelvin at which point it begins to decrease in resistance again. Each one of these prominent features is suppressed when the magnetic field is applied, resulting in a smooth curve. After the curve in that region, the resistance continues to decrease as the temperature goes down. This is 5 Kelvin less than the non-magnetic field run prior, and can be considered to have been shifted and suppressed. On the final run, the magnetic field was removed, and we see that the drop in resistance most likely occurred at a higher temperature then our system achieves during runs (see N Low, Peel 003, Run 003 - MF and Run 004 in Appendix 13 ). At 319 Kelvin, the drop of resistance stops and increases until 306 Kelvin. From there it decreases to 280 Kelvin at which point, it increases to 230 Kelvin where it then starts to decrease until the bottom temperature. Comparing run 002 and run 004, we see that they have very similar features and Run 004 has a slightly lower resistance which is overlapping with the magnetic field run. This shows a residual effect of the magnetic field which causes it to maintain a lower resistance. This can partly be explained by pancake vortices melting. The presence of a magnetic field causes a suppression of features which returned when the magnetic field is removed. However, a change in the current flow is induced by the magnetic field, seen in following runs.

The high dose implantation with a concentration in HOPG of $5.71 \mathrm{E}+16 / \mathrm{cm}^{3}$, did not have any signs of superconductivity (see N High in Appendix 14 ). There was a mild 
negative temperature coefficient but did not have any prominent steps or features. Lower doses of nitrogen ion implanted into HOPG warrants future investigation.

Beryllium is stripped of its 2 outermost electrons in the $S$ orbital, prior to ion implantation, to give it a crystal ionic radius of $59 \mathrm{pm}$. On impact with the HOPG surface, the charge neutralization process returns the electrons, giving it an atomic radius of $112 \mathrm{pm}$. At this radius, with an energy of $10 \mathrm{keV}$, the projected range is $442 \AA$ with a longitudinal straggle of $171 \AA$ and lateral straggle of $130 \AA$. When this bivalent atom comes to rest, it becomes an electron acceptor for neighboring carbon atoms since it has 2 less valence electron.

For the beryllium low-dose sample with a concentration in HOPG of $2.81 \mathrm{E}+13$ $/ \mathrm{cm}^{3}$, there are no signs of superconductivity present (see Be Low in Appendix 15 ). The curve is featureless with a positive temperature coefficient. For the high dosage with concentration in HOPG of $2.81 \mathrm{E}+16 / \mathrm{cm}^{3}$, the $R$ vs. $T$ response changed slightly, giving it a slightly negative temperature coefficient but still does not have any prominent features (see Be High in Appendix 16 ).

Fluorine is stripped of its 7 outermost electrons in the $\mathrm{S}$ and $\mathrm{P}$ orbitals, prior to ion implantation, to give it a crystal ionic radius of $22 \mathrm{pm}$. On impact with the HOPG surface, the charge neutralization process returns the electrons, giving it an atomic radius of $42 \mathrm{pm}$. At this radius, with an energy of $10 \mathrm{keV}$, the projected range is $193 \AA$ with a longitudinal straggle of $68 \AA$ and lateral straggle of $50 \AA$. When this univalent atom comes to rest, it has 3 more electrons than its neighboring carbon atoms but will accept an additional electron to fill its last orbital. 
Fluorine low dosage with a concentration in HOPG of $7.06 \mathrm{E}+13 / \mathrm{cm}^{3}$, has a very slight negative temperature coefficient that is featureless with no signs of superconductivity (see F Low in Appendix 17 ). The high dosage with concentration in HOPG of $7.06 \mathrm{E}+16 / \mathrm{cm}^{3}$, has a positive temperature coefficient but it is also featureless as well as having no signs of superconductivity (see F High in Appendix 18 ).

Lithium is stripped of its outermost electron in the $\mathrm{S}$ orbital, prior to ion implantation, to give it a crystal ionic radius of $90 \mathrm{pm}$. On impact with the HOPG surface, the charge neutralization process returns the electrons, giving it an atomic radius of $167 \mathrm{pm}$. At this radius, with an energy of $10 \mathrm{keV}$, the projected range is $612 \AA$ with a longitudinal straggle of $234 \AA$ and lateral straggle of $187 \AA$. This is the furthest range for species that we have tested. When this univalent atom comes to rest, it becomes an electron acceptor for neighboring carbon atoms since it has 3 less valence electron.

Lithium low dosage with a concentration in HOPG of $2.05 \mathrm{E}+13 / \mathrm{cm}^{3}$, has a very slight negative temperature coefficient that is featureless with no signs of superconductivity (see Li Low in Appendix 19). The high dosage with a concentration in HOPG of $2.05 \mathrm{E}+16 / \mathrm{cm}^{3}$, has a positive temperature coefficient but it is featureless with no signs of superconductivity (see Li High in Appendix 20 ).

Sodium is stripped of its outermost electron in the $\mathrm{S}$ orbital, prior to ion implantation, to give it a crystal ionic radius of $116 \mathrm{pm}$. On impact with the HOPG surface, the charge neutralization process returns the electrons, giving it an atomic radius of $190 \mathrm{pm}$. At this radius, with an energy of $10 \mathrm{keV}$, the projected range is $169 \AA$ with a longitudinal straggle of $58 \AA$ and lateral straggle of $43 \AA$. When this univalent atom 
comes to rest, it becomes an electron acceptor for neighboring carbon atoms since it has 3 less valence electron.

The low dosage of sodium ion implanted with a concentration in HOPG of $8.28 \mathrm{E}+13 / \mathrm{cm}^{3}$, has the most extreme negative temperature coefficient out of the samples we have seen (see Na Low in Appendix 21 ). At our highest temperature of 345 Kelvin, the resistance is 1.8 milliohms. The resistance continues to go up, as temperature goes down until 58 Kelvin where are the resistance is 3.1 milliohms. It then goes down to a final resistance of 2.7 milliohms at 3 Kelvin which is still a higher resistance then the highest temperature. This may have potential as a semiconductor or in other applications where such a negative temperature coefficient is desirable.

For the high sodium dosage with a concentration in HOPG of $8.28 \mathrm{E}+16 / \mathrm{cm}^{3}$, we see a small step at 235 Kelvin (see Na High in Appendix 22 ). Dosages in this range or higher may merit future investigation.

Sulfur is stripped of its 4 outermost electrons in the $\mathrm{S}$ and $\mathrm{P}$ orbitals, prior to ion implantation, to give it a crystal ionic radius of $51 \mathrm{pm}$. On impact with the HOPG surface, the charge neutralization process returns the electrons, giving it an atomic radius of $88 \mathrm{pm}$. At this radius, with an energy of $10 \mathrm{keV}$, the projected range is $125 \AA$ with a longitudinal straggle of $38 \AA$ and lateral straggle of $30 \AA$. When this hexavalent atom comes to rest, it becomes an electron donor for neighboring carbon atoms since it has 2 extra valence electrons.

Both low dosages and high dosages of sulfur which have a concentration in HOPG of $1.26 \mathrm{E}+14 / \mathrm{cm}^{3}$ and $1.26 \mathrm{E}+14 / \mathrm{cm}^{3}$ respectively, have a featureless negative 
temperature coefficient in their $R$ vs. $T$ curves (see S Low in Appendix 23 and S High in Appendix 24 ). No signs of superconductivity are present.

A response consistent with the presence of magnetic field flux vortices in phosphorous implanted Highly Oriented Pyrolytic Graphite and in phosphorous-doped exfoliated multilayer graphene has been observed. The melting of stacks of pancake vortices of varying lengths can account for the repeated nature of the observed steps in the Resistance versus Temperature characteristics of the material. The lack of zero resistance at low temperatures is also consistent with pancake vortex behaviour in the flux-flow regime. Thus the observed features can be described using the pancake vortex phenomenon. The presence of magnetic vortices requires, and is direct evidence of, superconductivity. The small Meissner effect may simply mean that the volume fraction of material involved is quite small or that the penetration depth is significantly larger than the sample thickness.

The material that was subjected to post doping argon implantation (damage) showed a discontinuous step in resistance at a temperature of about $265 \mathrm{~K}$. The initial findings from prior work [72], led to the conclusion that the ultimate critical temperature in this system under test was in excess of $100 \mathrm{~K}$ and, may have very likely been considerably higher if damage incurred during the doping process was minimized. Clearly this conclusion has not been voided and, based on the material with phosphine in the Plasma Chemical Vapor Deposition growth gas mixture, may well be valid for a transition at a temperature of greater than $300 \mathrm{~K}$.

It was observed that in addition to the first multilayer of Phosphine doped graphene, there was also present a great amount of graphite layered material adhering to the desired 
layers. This extra material may have the effect of acting as a parallel conductor, shunting the graphene transport to some degree, however we are unable to ascertain what effect this additional material has on the transport measurements with our current experimental apparatus.

\section{CONCLUSION}

By using several known superconductors, we were able to verify that our cryogenic system was working correctly. When testing the $\mathrm{YBa}_{2} \mathrm{Cu}_{3} \mathrm{O}_{7-\mathrm{x}}$ Target, we used indium between the Surface and probes. Even though this is not typically used for our HOPG and graphene samples, this did not change the measurement process and still allowed us to confirm proper system functionality. Another deviation was that we used 1 milliamp since $\mathrm{YBa}_{2} \mathrm{Cu}_{3} \mathrm{O}_{7-\mathrm{X}}$ is a low conducting ceramic until it becomes superconducting. This changed the sensitivity of our Delta measurements when compared to our HOPG and graphene samples but showed that our transition temperature was accurately at 88 Kelvin. The thickness of the sample ( $3 \mathrm{~mm}$ ) caused the top surface to cool slower than the bottom and led to a cooling transition temperature of 21 Kelvin. During cooling, which is 18 times faster than warming, this becomes more noticeable. For warming, this temperature gradient becomes negligible.

When testing the $\mathrm{YBa}_{2} \mathrm{Cu}_{3} \mathrm{O}_{7-\mathrm{x}}$ Hairpin filter which was 1 millimeter thick, we saw that there was a cooling transition temperature of 42 Kelvin and warming transition temperature of $92 \mathrm{Kelvin}$. Since the same current was used as the $\mathrm{YBa}_{2} \mathrm{Cu}_{3} \mathrm{O}_{7-\mathrm{X}}$ target, we can assume that heating caused by current is equal and the cooling transition temperature being closer to the warming transition temperature is solely due to the sample being 
thinner. The lower temperature gradient assured us that for thin graphene peels, this will be significantly lower.

When testing our element type II superconductor niobium, we found that our warming transition temperature was 9.244 Kelvin which is 0.056 Kelvin from its known transition temperature of 9.3 Kelvin. This further confirms that our thermocouples are working correctly and have high accuracy. We also saw that our system was capable of measuring an instantaneous drop in resistance even at very low temperatures. This one was also important for refining our set up to include a universal power supply which would continue measurements during power failures.

We learned that our Keithley Model 6221 will blink or source zero current with a blue indicator light on the front panel if there is an open circuit due to a loss of contact. The measurement will also drop to zero in voltage causing a erroneous reading in the resistance since there is no current running through the material. This happens virtually instantaneously due to thermal contraction. Although this was not desired, this shows us that small or gradual steps we were to see in future runs could not simply be explained by loss of contact.

For our low dose ion implantation of arsenic, we saw a change in slope at 255 Kelvin which continues to 148.77 Kelvin. There could be a very gradual transition occurring. With a magnetic field applied, the change in slope happened more gradually but did not create a change conclusive enough to determine if there is flux flow present. The higher dose of Arsenic ion implantation did not appear to have this effect. Exploring a lower dose of arsenic could potentially enhance the features we saw or simply 
minimizing the damage that could be lowering the coherence length, by using a Chemical Vapor Deposition arsenic graphene growth.

On our aluminum ion implanted sample with low dosage, we see an abrupt step at 264 Kelvin which ends at 210 Kelvin. When the magnetic field was applied, this step was suppressed by 14 Kelvin. The end of the transition was suppressed by 9 Kelvin. This shows a clear change caused by the magnetic field. On the following run, when the magnetic field was removed, there appears to be residual effects that cause the run to look identical to the magnetic field run. This can be explained by residual vortices that were induced by the prior run. For the higher dosage of arsenic, we do not see this step. This is most likely due to more damage in the sample. For future work, a stronger magnetic field could be applied to see if the step gets suppressed further. A higher grade of HOPG (ZYA) could be explored as well as Chemical Vapor Deposition growth with aluminum present.

The low dose ion implantation of nitrogen showed a negative temperature coefficient with a step a 283 Kelvin and ending at 261 Kelvin. When the magnetic field is applied, we see that instead of an abrupt step it changes to a gradual slope near the transition apex. Outside of that, the 2 runs are very close to each other and even overlapping at higher temperatures. When the magnetic field was removed, the following run was identical to the previous magnetic field run. There appears to be a residual effect caused by the magnetic field. For future work, a stronger magnetic field should be applied since there was not a strong change when the magnetic field we used was present. Upon further investigation, we did several peels to test the $2 \mathrm{D}$ characteristics that would closely approximate multi-layered grapheme [420]. There appeared to be 3 
prominent changes in the slope that were linearly connected. This multi-step region occurred between 329 Kelvin and 235 Kelvin. These steps disappear when the magnetic field is applied, and just turns to a smooth curve in that region. The overall resistance also lowers when the magnetic field is applied in that region butt on the following run when the magnetic field is removed, the features return and the overall resistance in that region remains identical to what it was with the field applied. This shows that there was a residual effect that occurred from the magnetic field that affected the resistance but still allowed the features to return. The fact that the magnetic field change the response warrants future investigation. The higher dose appears to be featureless due to excess damage.

For the beryllium low dose bulk sample, there are no features present. For the high dosage, it changes to a negative temperature coefficient but does not show any features. There are no signs of superconductivity present.

Fluorine had a slight negative temperature coefficient at low dosages but a positive temperature coefficient at high dosages. Both were featureless and did not show signs of superconductivity.

Lithium had a slight negative temperature coefficient at low dosages but a positive temperature coefficient at high dosages. Both were featureless and did not show signs of superconductivity.

For the low dosage of sodium, we see an extremely negative temperature coefficient. Even after the freeze out region, the resistance was higher then the resistance at 345 Kelvin. There were no features present and there are no signs of superconductivity. 
For the high dosage of sodium, we saw a small step at 235 Kelvin. This could be a transition for just several layers in the bulk HOPG that is being shunted by non superconducting layers in the HOPG. Testing with a magnetic field and removing several layers while testing each layer should be done to explore this feature.

Both low dosages and high dosages of sulphur yielded a negative temperature coefficient but did not show any features. There are no signs of superconductivity present.

For the phosporous doped samples the repeated steps in the resistance versus temperature characteristics can be seen to be independent of the manner in which the phosphorous-doped HOPG and graphene were doped. These steps become discontinuous at elevated temperatures when Argon (damage) is added. This is consistent with the results that are expected for thin superconducting films with and without damage.

The observed electrical resistance and magnetic behaviours of the samples under test can be seen to be consistent with superconductivity, even though a lack of zero resistance at low temperature was observed. In fact, the high degree of anisotropy in graphite and graphene predicts that if the material is a superconductor there would be significant magnetic flux-flow losses down to very low temperatures [68], [69]-[71].

The magnetic response of phosphorous-doped HOPG and graphene is as would be expected for superconductors of similar physical characteristics. That is (1) that the superconducting region is thin in comparison to the London (magnetic) penetration depth and (2) that the high level of anisotropy in the material is favourable for the formation of pancake vortices. The observed quenching of the $R$ vs. $T$ curve by application of a magnetic field is also to be expected for a superconductor. 
The magnetization and susceptibility results for phosphorous-doped HOPG and graphene suggests (1) a transition temperature above $260 \mathrm{~K}$ for the magnetization based upon the Zero-Field Cooled to Field Cooled Hysteresis loop and (2) a transition temperature above $150 \mathrm{~K}$ based upon the AC susceptometer measurements. There is no trace of ferromagnetism in the results as both the magnetization and susceptibility are negative in the lower temperature region.

In going from room temperature to a temperature of $80 \mathrm{~K}$, The Hall voltage has been observed to undergo a sign change during Hall Effect measurements. This phenomenon has been observed in other, known and accepted, superconductors that are in the flux-flow region of the mixed or vortex state [356]-[386]. It is also seen in anomalous ferromagnetism. Since there are (1) no ferromagnetic atoms and (2) no atoms with $\mathrm{d}$ or $\mathrm{f}$ electron shells in the sample, it is highly unlikely that ferromagnetism is involved in these Hall measurements. The fact that the magnetization and magnetic susceptibility are both negative suggests that ferromagnetism played no part in the sign reversal in the Hall voltage.

The aforementioned evidence forces us to conclude that phosphorous-doped Highly Oriented Pyrolytic Graphite (and phosphorous-doped graphene) is a superconductor with a transition temperature between $150 \mathrm{~K}$ and $260 \mathrm{~K}$. Some of the data acquired hints at a possibility that the transition temperature in the best samples may approach room temperature.

In summary, we were the first to systematically and exhaustively dope graphene for the purposes of creating a high temperature superconductor. We were the first to study the characteristics of Plasma Enhanced Chemical Vapor Deposited graphene in a 
phosphine environment due to our custom system built and certified for that purpose. We successfully built a cryogenic system that can achieve 2.5 Kelvin measurements, all the way up to 348 Kelvin, with a removable magnetic field coil system that does not disturb the sample. Detailed software was created from scratch to automate almost every aspect of our delta measurements to ensure consistency while also providing convenience. This software successfully controlled voltage measurements, current generation, compressor functionality and monitoring, temperature monitoring and heaters to allow above room temperature measurements. We were not successful in creating a high accuracy susceptometer due to ambient noise, however, we compensated by sending our samples to an external research facility to achieve the same goals.

- We have created the highest transition temperature on record, of upwards of 260 Kelvin with indications of near room temperature transitions using perfected doping techniques.

- All of the pentavalent electron donors showed signs of superconductivity (phosphorus, nitrogen, arsenic). These showed strongest features for low dosage.

- We saw that flux vortices were created in doped graphene, indicating it is a type II superconductor.

- As expected, Plasma Enhanced Chemical Vapor Deposition doping minimized the damage to our graphene lattice and allowed longer coherence lengths than ion implanted doping. This also gave us flux flow which added to our resistance and prevented us from seeing a zero resistance superconductor.

- Ion Implanted doping created columnar defects that pinned our flux vortices. This created pancake vortices that melted off in stages, causing multiple steps. 
- The mixed state region appeared to be much larger than we expected, with high temperature boundaries upwards of 260 Kelvin and low temperature boundaries unmeasurable by our equipment (may occur at temperatures under 3 Kelvin or not at all). This is the first time this phenomenon has been seen.

\section{REFERENCES}

[1] E. Benedict, T. Collins, D. Gotham, S. Hoffman, D. Karipides, S. Pekarek and R. and Ramabhadran, "LOSSES IN ELECTRIC," ECE Technical Reports, p. 266, 1992.

[2] G. Elert, "Electric Resistance," The Phsics Hypertextbook, 2012. [Online]. Available: http://physics.info/electric-resistance/. [Accessed 110 2012].

[3] John Bardeen, "Theory of the Meissner Effect in Superconductors." Physical Review. Vol. 97 No. 6 (March 1955) 1724-1725.

[4] Leon Cooper. "Bound Electron Pairs in a Degenerate Fermi Gas." Physical Review. Vol. 104 No. 4 (November 1956): 1189-1190.

[5] John Bardeen, Leon Cooper, John Robert Schrieffer. "Theory of Superconductivity." Physical Review. Vol. 108 No. 5 (December 1957): 11751204.

[6] John Bardeen. "Two-Fluid Model of Superconductivity." Physical Review Letters. Vol. 1 (1958): 399-400.

[7] Nobel Prize in Physics 1987 to Bednorz \& Müller "for their important breakthrough in the discovery of superconductivity in ceramic materials".

[8] Possible high $\mathrm{T}_{\mathbf{c}}$ superconductivity in the $\mathrm{Ba}-\mathrm{La}-\mathrm{Cu}-\mathrm{O}$ system. J.G. Bednorz and K.A. Müller. Zeitschrift für Physik B Condensed Matter. Vol. 64 No. 2 (June 1986): 189-193

[9] Bulk superconductivity at $36 \mathrm{~K}_{\text {in }} \mathrm{La}_{1.8} \mathrm{Sr}_{0.2} \mathrm{CuO}_{4}$. R.J. Cava, R.B. van Dover, B. Batlogg, and E.A. Rietman.Physical Review Letters. Vol. 58 No. 4 (26 January 1987): 408-410. 
[10] Superconductivity at $93 \mathrm{~K}$ in a new mixed-phase $\mathrm{Yb}-\mathrm{Ba}-\mathrm{Cu}-\mathrm{O}$ compound system at ambient pressure. M.K. Wu, J. R. Ashburn, C.J. Torng, P.H. Hor, R.L. Meng, L. Gao, Z.J. Huang, Y.Q. Wang, and C.W. Chu. Physical Review Letters. Vol. 58 No. 9 (2 March 1987): 908-910. This was the most cited scientific paper of 1987 and the 6th most cited of the 1980s despite containing an intentional typo.

[11] Superconductivity above $130 \mathrm{~K}$ in the $\mathrm{Hg}-\mathrm{Ba}-\mathrm{Ca}-\mathrm{Cu}-\mathrm{O}$ system. A. Schilling,et al. Nature. Vol. 363 (6 May 1993): 56-58.

[12] Superconductivity up to $164 \mathrm{~K}$ in $\mathrm{HgBa}_{2} \mathrm{Ca}_{-1} \mathrm{Cu} \mathrm{O}_{2} m_{+2+\delta}$ under quasihydrostatic pressures. L. Gao,et al. Physical Review B. Vol. 50 No. 6 (1 August 1994): 4260-4263.

[13] (2001)Nagamatsu, Nakagawa, Muranaka, Zenitani Akimitsu Nagamatsu2001 Nagamatsu, J., Nakagawa, N., Muranaka, T., Zenitani, Y. Akimitsu, J. Superconductivity at $39 \mathrm{~K}$ in magnesium diboride. ph Nature 410 , 63 (2001).

[14] Conventional superconductivity at 203 kelvin at high pressures in the sulfur hydride system. A.P. Drozdov, M.I. Eremets, I.A. Troyan, V. Ksenofontov \& S.I. Shylin. Nature. Vol. 525 No. 7567 (3 September 2015): 73-76.

[15] R. Kumar, K. Farkas, N. P. Jouppi, P. Ranganathan and D. M. Tullsen, "A Multi-Core Approach to Addressing the Energy-Complexity Problem in Microprocessors," In Proceedings of the Workshop on Complexity-Effective Design(WCED), pp. 1-8, June 2003.

[16] Integrated Device Technology, "64 Bit RISC Microprocessor.” 79R4700 data sheet. April 10, 2001.

[17] Digital Equipment Corporation. "Digital Semiconductor Alpha 21064 and Alpha 21064A Microprocessors.” Alpha 21064 and Alpha 21064A Hardware Reference Manual. June 1996.

[18] Digital Equipment Corporation. "Alpha 21164 Microprocessor”. Alpha 21164 Microprocessor:Hardware Reference Manual. July 1996

[19] Compaq Computer Corporation. "Alpha 21264 Microprocessor Hardware Reference Manual.” July 1999.

[20] Semiconductor Industry. "2001 Technology Roadmap for Semiconductors." January 2002. 
[21] D. H. Albonesi. "Selective cache-ways: On demand cache resource allocation." In IEEE/ACM International Symposium on Microarchitecture (MICRO-32), 1999.

[22] A. M. Despain and J.-L. Gaudiot. "HIDISC: A decoupled architecture for applications in data intensive computing." May 2001.

[23] D. Folegnani and A. Gonzalez. "Reducing power consumption of the issue logic." In Proceedings of the Workshop on Complexity-Effective Design. June 2000 .

[24] S. Ghiasi, J. Casmira, and D. Grunwald. "Using IPC variation in workloads with externally specified rates to reduce power consumption." In Workshop on Complexity Effective Design. June 2000.

[25] K. Govil, E. Chan, and H. Wasserman. "Comparing algorithms for dynamic speed-setting of a low-power cpu." In 1st Int'l Conference on Mobile Computing and Networking. Nov. 1995.

[26] D. Grunwald, A. Klauser, S. Manne, and A. Pleskun. "Confidence estimation for speculation control.” In 25th Annual International Symposium on Computer Architecture. June 1998.

[27] A. Iyer and D. Marculescu. "Power aware microarchitecture resource scaling." In Proceedings of IEEE Design, Automation and Test in Europe Confeence(DATE). March 2001.

[28] A. Klauser. "Trends in high-performance microprocessor design." In Telematik2001. January 2001.

[29] A. Kumar. “The HP PA-8000 RISC CPU.” In Hot Chips VIII. Aug. 1996.

[30] S. Manne, A. Klauser, and D. Grunwald. "Pipeline gating: Speculation control for energy reduction." In 25th Annual International Symposium on Computer Architecture. June 1998.

[31] R. Maro, Y. Bai, and R. Bahar. "Dynamically reconfiguring processor resources to reduce power consumption in high performance processors." In PACS. 2000

[32] J. M. Mulder, N. T. Quach, and M. J. Flynn. "An area model for on-chip memories and its applications." In IEEE Journal of Solid State Circuits. February 1991. 
[33] T. Pering, T. Burd, and R. Brodersen. "The simulation and evaluation of dynamic voltage scaling algorithms." In Proceedings of 1998 International Symposium on Low Power Electronics and Design. Aug. 1998

[34] J. M. Rabaey. "The quest for ultra-low energy computation opportunities for architectures exploiting low-current devices." April 2000.

[35] T. Sherwood and B. Calder. "Time varying behavior of programs." In UC San Diego Technical Report UCSD-CS-99- 630. August 1999.

[36] T. Sherwood, E. Perelman, G. Hammerley, and B. Calder. "Automatically characterizing large-scale program behavior." In Proceedings of the International Conference on 10th International Conference on Architectural Support for Programming Languages and Operating Systems. October 2002.

[37] P. Shivakumar and N. Jouppi. "CACTI 3.0: An integrated cache timing, power and area model." In Technical Report 2001/2, Compaq Computer Corporation. August 2001.

[38] D. Tullsen. "Simulation and modeling of a simultaneous multithreading processor." In 22nd Annual Computer Measurement Group Conference. December 1996.

[39] D. Tullsen, S. Eggers, and H. Levy. "Simultaneous multithreading: Maximizing on-chip parallelism." In 22nd Annual International Symposium on Computer Architecture. June 1995.

[40] D. Wall. "Limits of instruction-level parallelism." In Fourth International Conference on Architectural Support for Programming Languages and Operating Systems. pages 176- 188, April 1991.

[41] S. Wilton and N. Jouppi. "CACTI: an enhanced cache access and cycle time model.” In IEEE Journal of Solid State Circuits, Vol 31, No. 5. May 1996.

[42] S. N. Song and J. B. Ketterson, Superconductivity, Cambridge University Press, 1999.

[43] "Superconducting Magnets," hyperphysics, [Online]. Available: http://hyperphysics.phy-astr.gsu.edu/hbase/solids/scmag.html. [Accessed 110 2012].

[44] "Solid State References," HYPERPHYSICS.PHY-ASTR.GSU.EDU, [Online]. Available: htt://www.hyperphysics.phy-astr.gsu.edu 
[45] "Superconductors," SUPERCONDUCTORS.ORG, [Online]. Available: http://www.superconductors.org.

[46] "Superconductivity Papers Database." SPRCND.CLOUDAPP.NET [Online]. Available: http://sprcnd.cloudapp.net

[47] Institute of Physics, "Superconductor Science and Technology.” June 2016

[48] Heike Kamerlinght Onnes, "Investigrations into the Properties of Substances at Low Temperatures, which Have Led, amongst Othert Things, to the Preparation of Liquid Helium.” December 1913.

[49] John Bardeen, Leon Cooper, and John Schreiffer, "Theory of Superconductivity. The BCS-theory." 1972.

[50] Brian D. Josephson, Leo Esaki, Ivar Giaever, "The Discovery of Tunnelling Supercurrents.” December 1973.

[51] Alexei A. Abrikosov, Vitaly L. Ginzburg, Anthony J. Legget, "Theory of Superconductors and Superfluids." 2003

[52] Robert W. Dull, "A Teacher's Guide to Superconductivity for High School Students." September 1994.

[53] "Graphene - the perfect atomic lattice," Nobelprize.org, 5 October 2010. [Online]. Available: http://www.nobelprize.org/nobel_prizes/physics/laureates/2010/press.html. [Accessed 110 2012].

[54] "The Nobel Prize in Physics 2010 - Speed Read," Nobelprize.org, 5 october 2010. [Online]. Available: http://www.nobelprize.org/nobel_prizes/physics/laureates/2010/speedread.html.

[55] B. Kessler, C. Girit, A. Zettl and V. Bouchiat, Phys. Rev. Lett, vol. 104, no. 047001, p. 4, 2010.

[56] H. B. Heersche, P. Jarillo-Herrero, J. B. Oostinga, L. M. K. Vandersypen and a. A. F. Morpurgo, "Bipolar Supercurrent in Graphene," Nature, vol. 446, pp. 5659, 2007.

[57] X. Du, I. Skachko and a. E. Y. Andrei, "Josephson Current and Multiple Andreev Reflections in Graphene SNS Junctions," Phys. Rev., vol. B, no. 184507, p. 77, 2008. 
[58] A. Ballestar, J. Barzola-Quiqui and a. P. Esquinazi, "Evidence of Josephsoncoupled superconducting regions at the interfaces of Highly Oriented Pyrolytic Graphite," arXiv:1206.2463 [cond-mat.supr-con], p. 5, 13 Jun 2012.

[59] N. B. Hannay, T. H. Geballe, B. T. Matthias, K. Andres, P. Schmidt, and D. MacNair, "Superconductivity in Graphitic Compoungs." Phys. Rev. Lett. 14, 7. February 1965.

[60] R. Ricardo da Silva, J. H. S. Torres, Y. Kopelevich, "Indication of Superconductivity at $35 \mathrm{~K}$ in Graphite-Sulfur Composites." Phys. Rev. Lett. 87, 147001. September 2001.

[61] A. Ballestar, J. Barzola-Quiquia, P. Esquinazy, "Evidenve of Josephson-coupled superconducting regions at the interface of Highly Oriented Pyrolytic Graphite." June 2012.

[62] I. Felner, O. Wolf, O. Millo, "Superconductivity in Sulfer-Doped Amorphous Carbon Films." July 2009.

[63] J. Gonz'alez, F. Guinea, and M. A. H. Vozmediano, "Electron-electron interactions in graphene sheets." Phys. Rev. B 63, 134421. March 2001.

[64] A. M. Black-Schaffer and S. Doniach, "Resonating valence bonds and meanfield d-wave superconductivity in graphite." Phys. Rev. B 75, 134512. April 2007.

[65] N. B. Kopnin, T. T. Heikkil"a, and G. E. Volovik, "High-temperature surface superconductivity in topological flat-band systems." Phys. Rev. B 83, 220503 June 2011.

[66] N. Garcia and P. Esquinazi, "Mean Field Superconductivity Approach in Two Dimensions." November 2009.

[67] T. E. Weller, M. Ellerby, S. S. Saxena, R. P. Smith and N. T. Skipper, "Superconductivity in the Intercalated Graphite Compounds C6Yb and C6Ca," Nature Phys., vol. 39, no. 1, 2005.

[68] N. Emery, C. Herold, M. D'Astuto, V. Garcia, C. Bellin, J. F. Mareche, P. Lagrange and a. G. Loupias, "Superconductivity of bulk CaC6," Phys. Rev., vol. 95, no. 087003, 2005.

[69] A. Posazhennikova, "Josephson effect in Superconductors and Superfluids," 12 Aug 2009.

[70] B. D. Josephson, "Possible New Effects in Superconductie Tunnelling." Phys. Lett. 1, 251. June 1962. 
[71] A. Barone, G. Paterno, "Physics and Applications of the Josephson Effect." 1982.

[72] G. L. a. Y. Vlasov, "Indications of superconductivity in doped highly oriented pyrolytic graphite," Supercond. Sci. Technol., vol. 24, no. 092001, p. 4, 2011.

[73] K. V. G, Phys. Rev. B, vol. 75, no. 064514, 2007.

[74] U. B and a. C. N. A. H, Phys. Rev. Lett., vol. 98, no. 146801, 2007.

[75] B.-S. A. M and a. D. S, Phys. Rev. B, vol. 75, no. 134512, 2007.

[76] B. A, O. T, M. J. L, S. T and H. K. a. R. E, Solid State Commun., vol. 143, no. 63-71, 2007.

[77] P. E. a. G. J, Physica, vol. 40, no. 1039, pp. 460-462, 2007.

[78] P. S and S. V. B. a. B. G, no. arXiv:0809.0244, 2008.

[79] "HOPG: Highly Ordered Pyrolytic Graphite," MikroMasch, [Online]. Available: http://www.spmtips.com/HOPG. [Accessed 310 2012].

[80] T. P. Inc, "Pelco: Technical Notes - Highly Ordered Pyrolytic Graphite," [Online]. Available: http://www.tedpella.com/technote_html/626\%20TN.pdf. [Accessed 110 2012].

[81] "Highly Orientated Pyrolytic Graphite," tec tra, 277 1999. [Online]. Available: http://www.tectra.de/HOPG.htm.

[82] "Materials and Components," MOMENTIVE, [Online]. Available: http://www.momentive.com/categories/product-types-productslist.aspx? categoryId $=4294967335$.

[83] "High quality HOPG for scientific research and graphite HOPG monochromators," $\quad$ SPI supplies, [Online]. Available: http://www.2spi.com/catalog/new/HOPGsub.php. [Accessed 110 2012].

[84] "HOPG," [Online]. Available: http://nanoprobes.aistnt.com/apps/HOPG\%20info.htm.

[85] "KaptonTape.com," KaptonTape.com, [Online]. Available: http://www.kaptontape.com/. [Accessed 110 2012].

[86] Keithley, "Four-Probe Resistivity and Hall Voltage," Application Note Series, no. 2475, p. 8, 2011 
[87] Keithley, "Measuring the Resistivity and Determining the Conductivity Type of Semiconductor Materials Using a Four-Point Collinear Probe and the Model 6221 DC and AC Current Source," Application Note Series, no. 2615, 2005.

[88] Keithley, "Low-Level Pulsed Electrical Characterization with the Model 6221/2182A Combination," Application Notes Series, no. 2611, 2004.

[89] "Four Point Probe," University of Illinois Urbana-Champaign - Department of Electrical and Computer Engineering , 2012. [Online]. Available: http://fabweb.ece.uiuc.edu/gt/equations/fpp.aspx. [Accessed 110 2012].

[90] M. S. R. a. M. Salim, "Design aspects of a differential magnetic," Meas. Sci. Technol., vol. 12, no. 744, 2001.

[91] M. S. Dresselhaus, G. Dresselhaus, and P. C. Eklund, Science of fullerenes and carbon nanotubes, Academic Press. San Diego, CA, 1996.

[92] K. S. Novoselov, A. K. Geim, S. V. Morozov, Z. D. Jiang, Y. Zhang, V. Dubonos, I. V. Grigorieva, and A. A. Firsov, "Electric Field Effect in Atomically Thin Carbon Films,” Science, vol. 306, pp. 666-669, 2004.

[93] C. D. Dimitrakopoulos, D. J. Mascaro, "Organic thin-film transistors: A review of recent advances." IBM J. Res. Dev. 45, 11. January 2001.

[94] R. H. Baughman, A. A. Zakhidov, de Heer W.A., "Carbon nanotubes-the route toward applications." Science 297, 787. August 2002.

[95] S. V. Rotkin, K. Hess, "Possibility of a metallic field-effect transistor." Appl. Phys. Lett. 84, 3139. February 2004.

[96] A. V. Butenko, D. Shvarts, V. Sandomirsky, Y. Schlesinger, "Hall constant in quantum-sized semimetal Bi films: Electric field effect influence." J. Appl. Phys. 88, 2634. June 2000.

[97] M. S. Dresselhaus, G. Dresselhaus, "Raman spectroscopy as a proble of graphene and carbon nanotubes." Adv. Phys. 51, 1. January 2008.

[98] P. L. Walker, P. A. Thrower, "I. L. Spain, in Chemistry and Physics of Carbon." pp. 119-304.1981.

[99] O. A. Shenderova, V. V. Zhirnov, D. W. Brenner, "Critical Reviews on Solid State Mater." Sci. 27, 227. 2002.

[100] A. Krishnan et al., "Graphitic cones and the nucleation of curved carbon surfaces." Nature 388, 451. 1997. 
[101] E. Dujardin, T. Thio, H. Lezec, T. W. Ebbesen, "Electrical conductivity of individual carbon nanotubes." Appl. Phys. Lett. 79, 2474. July 1996.

[102] H. Shioyama, J. Mat. "Cleavage of graphite to graphene." Sci. Lett. 20, 499 March 2001.

[103] A. M. Affoune et al., "Experimental evidence of a single nano-graphene." Chem. Phys. Lett. 348, 17. November 2001.

[104] K. Harigaya, Y. Kobayashi, K. Takai, J. Ravier, T. Enoki, "Novel electronic wave interference patterns in nanographene sheets." J. Phys. Cond. Matter 14, L605. August 2002.

[105] T. A. Land, T. Michely, R. J. Behm, J. C. Hemminger, G. Comsa, "STM investigration of single layer graphite structures produced on Pt (111) by hydrogen decomposition." Surf. Sci. 264, 261. March 1992.

[106] Franklin N.R., Zhou C., Chapline M.G., Oeng S., Cho K. Dai H., J. Kong et al., "Nanotube molecular wires as chemical sensors." Science 287, 622. January 2000.

[107] M. Kru"ger, L. Forro, T. Nussbaumer, M. Buitelaar, C. Scho"nenberger, "The electrochemical carbon nanotube field-effect transistor." September 2000.

[108] M. R. Stan, P. D. Franzon, S. C. Goldstein, J. C. Lach, M. M. Zeigler, "Molecular electronics: from devices and interconnect to circuits and architecture." Proc. IEEE 91, 1940. November 2003.

[109] J. Hass, W. A. de Heer, and E. H. Conrad, "The growth and morphology of epitaxial multilayer graphene," J. Phys. Condens. Matter, vol. 20, no. 32, p. 323202, 2008.

[110] J. Hass, F. Varchon, J. E. Millán-Otoya, M. Sprinkle, N. Sharma, W. A. de Heer, C. Berger, P. N. First, L. Magaud, and E. H. Conrad, "Why multilayer graphene on $4 \mathrm{H}-\mathrm{SiC}(0001)$ behaves like a single sheet of graphene," Phys. Rev. Lett., vol. 100, no. 12, p. 125504, 2008.

[111] A. Bostwick, T. Ohta, J. L. McChesney, T. Seyller, K. Horn, and E. Rotenberg, "Renormalization of graphene bands by many-body interactions," Explor. Graphene Recent Res. Adv., vol. 143, no. 1-2, pp. 63-71, 2007.

[112] S. Pathak, V. B. Shenoy, and G. Baskaran, "Possibility of High Tc Superconductivity in doped Graphene," arXiv:0809.0244, 2008. 
[113] J.G. Bednorz, K.A. Muller, "Possible high Tc superconducvity in the Ba-La-CuO system.” Z Phys. B-Condensed Matter, 64, 189-193. June 1986.

[114] Anderson P.W., "The Resonating Valence Bond State in $\mathrm{La} 2 \mathrm{CuO} 4$ and Superconductivity." March 1987.

[115] J. Nagamatsu, N. Nakagawa, T. Muranaka, Y. Zenitani, J. Akimitsu, "Superconductivity at 39K in magnesium diboride." Letters to Nature. February 2001.

[116] Y. Kamihara, T. Watanabe, M. Hirano, "Iron-Based Layered Superconductor $\mathrm{LA}\left[\mathrm{O}_{1-\mathrm{x}} \mathrm{F}_{\mathrm{x}}\right] \mathrm{Fe} A \mathrm{~s}(\mathrm{x}=0.05-0.12)$ with $\mathrm{Tc}=26 \mathrm{~K} . "$ January 2008.

[117] A.K. Geim, K.S. Novoselov, "The rise of graphene.” June 2007.

[118] M.I. Katsnelson, "Graphene: carbon in two dimensions." arXiv:condmat/0612534. February 2007.

[119] A.H. Castro Neto, F. Guinea, N.M.R Peres, K.S. Novoselov, A.K. Geim, "The electronic properties of graphene." Rev. Mod. Phys. 81, 109. February 2008.

[120] G. Baskaran, "Resonating-valence-bond contribution to superconductivity in MgB.$"$ Phys. Rev. B 65, 212505. May 2002.

[121] A.M. Bkack-Schaffer, S. Doniach, "Resonating valence bonds and mean-field d-wave superconductivity in graphite." Phys. Rev. B 75, 134512. April 2007.

[122] B. Uchoa, A.H. Castro Neto, "Superconducting States of Pure and Doped Graphene." Phys. Rev. Lett. 98, 146801. April 2007.

[123] T.C. Choy, B.A. McKinnon, "Significance of nonorthogonality in tight-binding models. II. The possibility of high- Tc superconductivity in intercalation compounds." Phys. Rev. B 52. 24539. November 1995.

[124] N. Furukawa, "Antiferromagnetism of the Hubbard Model on a Layered Honeycomb Lattice- Is $\mathrm{MgB}_{2}$ a Nearly-Antiferromagnetic Metal?." J. Phys. Soc. Jpn. 70, pp. 1483-1486. March 2001.

[125] S. Onari, R. Arita, K. Kuroki, H. Aoki, "Superconductivity in repulsive electron systems with three dimensional disconnected Fermi surfaces." Phys. Rev. B 68, 024525. March 2003.

[126] D. Baeriswyl, E. Jackelman, "In The Hubbard Model: Its Physics and Mathematical Physics." NATO ASI series. Series B, Physics. 1995. 
[127] G. Baskaran, S.A. Jafari, "Gapless Spin-1 Neural Collective Mode Branch for Graphite." Phys. Rev. Lett. 89, 016402. June 2002.

[128] L. Pailing, "Nature of the chemical bond." Cornell University. January 1960.

[129] D. Ceperley, G. Chester, M. Kalos, "Monte Carlo simulation of a many-fermison study." Phys. Rev. B 16, 3081. October 1977.

[130] M.C. Gutzwiller, "Correlated electrons in a narrow band." Phys. Rev. 137, A1726-1735. March 1965

[131] A. Paramekanti, M. Randeria, N. Trivedi, "High Tc superconductors: A variational theory of the superconducting state." Phys. Rev. B 70, 054504. August 2004.

[132] S. Florens, A. Georges, "Slave-rotor mean field theories of strongly correlated systems and the Mott transition in finite dimensions." Phys. Rev. B 70, 035114. April 2004.

[133] E. Zhao, A. Paramekanti, "Self-consistent slave rotor mean-field theory for strongly correlated systems." Phys. Rev. B76, 195101. November 2007.

[134] C. Honerkamp, "Density Waves and Cooper Pairing on the Honeycomb Lattice." Phys. Rev. Lett. 100, 146404. April 2008.

[135] R. Ricardo da Silva, J.H.S Torres, and Y. Kopelevich, "Indication of Superconductivity at 35K in Graphite-Sulfur Composites." Phys. Rev. Lett. 87, 147001. September 2001.

[136] E. Perfetto and J. González, "Superconductivity in multi-walled carbon nanotubes and doped graphite," Proc. 8th Int. Conf. Mater. Mech. Supercond. High Temp. Supercond. M2S-HTSC VIII, vol. 460-462, pp. 1039-1040, 2007.

[137] Y. Kopelevich, P. Esquinazi, J. H. S. Torres, and S. Moehlecke, "Ferromagnetic- and Superconducting-Like Behavior of Graphite," J. Low Temp. Phys., vol. 119, no. 5-6, pp. 691-702, 2000.

[138] H. Kempa, P. Esquinazi, and Y. Kopelevich, "Field-induced metal-insulator transition in the c-axis resistivity of graphite," Phys. Rev. B, vol. 65, no. 24, p. 241101, 2002.

[139] Y. Kopelevich and P. Esquinazi, "Ferromagnetism and Superconductivity in Carbon-based Systems," J. Low Temp. Phys., vol. 146, no. 5-6, pp. 629-639, 2007. 
[140] T. Scheike, W. Böhlmann, P. Esquinazi, J. Barzola-Quiquia, A. Ballestar, and A. Setzer, "Can Doping Graphite Trigger Room Temperature Superconductivity? Evidence for Granular High-Temperature Superconductivity in Water-Treated Graphite Powder," Adv. Mater., vol. 24, no. 43, pp. 58265831, 2012.

[141] V. J. Kauppila, T. Hyart, T. T. Heikkilä, "Collective amplitude mode fluctuations in a flat band superconductor formed at a semimetal surface," Physical Review B, 93, 2, 2016.

[142] Grover Larkins, Yuriy Vlasov, Kiar Holland, "Evidence of superconductivity in doped graphite and graphene," Superconductor Science and Technology, 29, 1, $015015,2016$.

[143] A. N. Ionov, "Josephson-Like Behaviour of the Current-Voltage Characteristics of Multi-Graphene Flakes Embedded in Polystyrene," Journal of Low Temperature Physics, 2016.

[144] Shahid Ameer, Iftikhar Hussain Gul, Nasir Mahmood, Muhammad Mujahid, "Semiconductor-to-metallic flipping in a $\mathrm{ZnFe2O4-graphene} \mathrm{based} \mathrm{smart} \mathrm{nano-}$ system: Temperature/microwave magneto-dielectric spectroscopy," Materials Characterization, 99, 254, 2015.

[145] Annica M Black-Schaffer, Carsten Honerkamp, "Chirald-wave superconductivity in doped graphene," Journal of Physics: Condensed Matter, 26, 42, 423201, 2014.

[146] A M Panich, V Yu Osipov, K Takai, "Diamagnetism of carbon onions probed by NMR of adsorbed water," New Carbon Materials, 29, 5, 392, 2014.

[147] Tsuyoshi Takami, "Functional Cobalt Oxides," Fundamentals, Properties, and Applications, 45, 2014.

[148] Gufei Zhang, Stuart Turner, Evgeny A. Ekimov, Johan Vanacken, Matias Timmermans, Tomás Samuely, Vladimir A. Sidorov, Sergei M. Stishov, Yinggang Lu, Bart Deloof, Bart Goderis, Gustaaf Van Tendeloo, Joris Van de Vondel, Victor V. Moshchalkov, "Global and Local Superconductivity in Boron-Doped Granular Diamond," Advanced Materials, 26, 13, 2034, 2014.

[149] A. Cabo Montes De Oca, N. H. March, A. Cabo-Bizet, "Hartree-Fock Solutions of 2D Interacting Tight-Binding Electrons: Mott Properties and Room Temperature Superconductivity Indications," International Journal of Modern Physics B, 28, 04, 1450027, 2014. 
[150] Stuart C Wimbush, "Layers of intrigue: superconductivity at $4.4 \mathrm{~K}$ in Ba2Bi3," Superconductor Science and Technology, 27, 7, 070501, 2014.

[151] P. Esquinazi, T. T. Heikkilä, Y. V. Lysogorskiy, D. A. Tayurskii, G. E. Volovik, "On the superconductivity of graphite interfaces," JETP Letters, 100, 5, 336, 2014.

[152] A. Ballestar, P. Esquinazi, J. Barzola-Quiquia, S. Dusari, F. Bern, R.R. da Silva, Y. Kopelevich, "Possible superconductivity in multi-layer-graphene by application of a gate voltage," Carbon, 72, 312, 2014.

[153] Gianluca A. Artioli, Lorenzo Malavasi, "Superconductivity in metalintercalated aromatic hydrocarbons," Journal of Materials Chemistry C, 2, 9, 1577, 2014.

[154] N. B. Kopnin, M. Ijäs, A. Harju, T. T. Heikkilä, "High-temperature surface superconductivity in rhombohedral graphite," Physical Review B, 87, 14, 2013.

[155] Yasushi Kawashima, "Possible room temperature superconductivity in conductors obtained by bringing alkanes into contact with a graphite surface," AIP Advances, 3, 5, 052132, 2013.

[156] I. Felner, O. Wolf, O. Millo," Superconductivity in Sulfur-Doped Amorphous Carbon Films," Journal of Superconductivity and Novel Magnetism, 26, 3, 511, 2013.

[157] W. A. Muñoz, L. Covaci, F. M. Peeters, "Tight-binding description of intrinsic superconducting correlations in multilayer graphene," Physical Review B, 87, 13, 2013.

[158] Lianlian Chen, Liwei Guo, Zhilin Li, Han Zhang, Jingjing Lin, Jiao Huang, Shifeng Jin, Xiaolong Chen, "Towards intrinsic magnetism of graphene sheets with irregular zigzag edges," Scientific Reports, 3, 2013.

[159] T. Scheike, P. Esquinazi, A. Setzer, and W. Böhlmann, "Granular superconductivity at room temperature in bulk highly oriented pyrolytic graphite samples," Carbon, vol. 59, no. 0, pp. 140-149, 2013.

[160] A. Ballestar, J. Barzola-Quiquia, T. Scheike, and P. Esquinazi, "Josephsoncoupled superconducting regions embedded at the interfaces of highly oriented pyrolytic graphite," New J. Phys., vol. 15, no. 2, p. 023024, 2013.

[161] K. S. Novoselov, A. K. Geim, S. V. Morozov, D. Jiang, M. I. Katsnelson, I. V. Grigorieva, S. V. Dubonos, and A. A. Firsov, "Two-dimensional gas of 
massless Dirac fermions in graphene," Nature, vol. 438, no. 7065, pp. 197-200, 2005.

[162] K. Geim and K. S. Novoselov, "The rise of graphene," Nat. Mater., vol. 6, no. 3, pp. 183-191, 2007.

[163] H. Castro Neto, F. Guinea, N. M. R. Peres, K. S. Novoselov, and A. K. Geim, "The electronic properties of graphene," Rev. Mod. Phys., vol. 81, no. 1, pp. 109-162, 2009.

[164] Delft, D.; Kes, P. "The Discovery of Superconductivity," Physics Today: September 2010.

[165] V.L. Ginzburg, E.A. Andryushin. Superconductivity. World Scientific, 2004.

[166] "Type 1 Superconductors and a Periodic Chart Comparison," Superconductors, [Online]. Available: http://www.superconductors.org/TYPE1.htm\#AuAgCu. [Accessed 119 2015].

[167] Meissner, W.; R. Ochsenfeld, "Ein neuer Effekt bei Eintritt der Supraleitfähigkeit," Naturwissenschaften 21 (44): 787-788, 1933.

[168] Lev D. Landau; Evgeny M. Lifschitz. Electrodynamics of Continuous Media. Course of Theoretical Physics 8. Oxford: Butterworth-Heinemann, 1984.

[169] David J. E. Callaway, "On the remarkable structure of the superconducting intermediate state," Nuclear Physics B 344 (3): 627-645, 1990.

[170] J. Bardeen; L. N. Cooper; J. R. Schrieffer, "Theory of superconductivity," Physical Review B 106 (1175): 162-164, 1957.

[171] J.E Hirsch, "The origin of the Meissner effect in new and old superconductors," Physica Scripta 85: 035704, 2012.

[172] London, F., "On the Problem of the Molecular Theory of Superconductivity," Physical Review 74 (5): 562-573, September 1948.

[173] Bardeen, J., "Theory of the Meissner Effect in Superconductors," Physical Review 97 (6): 1724-1725, March 1955.

[174] Cooper, Leon, "Bound Electron Pairs in a Degenerate Fermi Gas," Physical Review 104 (4): 1189-1190, November 1956.

[175] Bardeen, J.; Cooper, L. N.; Schrieffer, J. R., "Microscopic Theory of Superconductivity," Physical Review 106 (1): 162-164, April 1957. 
[176] Mann, A., "High Temperature Superconductivity at 25: Still In Suspense," Nature 475 (7356): 280-2, July 2011.

[177] M. Tinkham, Introduction to superconductivity. Mineola, N.Y.: Dover Publications, 2004.

[178] "Type 2 Superconductors", Superconductors.org, 2015. [Online]. Available: http://www.superconductors.org/type2.htm. [Accessed: 14- Jul- 2015].

[179] Hartmann, Hellmuth; Ebert, Fritz; Bretschneider, Otto, "Elektrolysen in Phosphatschmelzen. I. Die elektrolytische Gewinnung von $\alpha$ - und ? $\beta$-Wolfram," Zeitschrift für anorganische und allgemeine Chemie 198: 116, 1931.

[180] Kiss, A. B., "Thermoanalytical Study of the Composition of $\beta$-tungsten," Journal of Thermal Analysis and Calorimetry 54 (3): 815, 1998.

[181] Boren, B., "X-Ray Investigation of Alloys of Silicon with Chromium, Manganese, Cobalt and Nickel," Ark. Kern., Min. Geol 11A (10): 2-10, 1933.

[182] Hardy, George; Hulm, John, "Superconducting Silicides and Germanides," Physical Review 89 (4): 884, 1953.

[183] Izyumov, Yurii A; Kurmaev, Z Z, "Physical properties and electronic structure of superconducting compounds with the $\beta$-tungsten structure," Soviet Physics Uspekhi 17 (3): 356, 1974.

[184] Sheahen, Thomas P, Introduction to high-temperature superconductivity. p. 32, 1994.

[185] "Limits of NbTi and Nb3Sn, and Development of W\&R Bi-2212 High Field Accelerator Magnets," Office of Science, High Energy Physics, U.S. Department of Energy. 2006-09-01. Retrieved 2015-12-26.

[186] "Record current with powder-in-tube superconductor," laboratorytalk.com. Retrieved 2008-09-06.

[187] R. Scanlan; A.F. Greene \& M. Suenaga, "Survey Of High Field Superconducting Material For Accelerator Magnets," SSC-MAG-81 ; LBL21549, May 1986.

[188] J.L.H., Lindenhovius; Hornsveld, E.M.; den Ouden, A.; Wessel, W.A.J.; ten Kate, H.H.J., "Powder-in-tube (PIT) Nb3Sn conductors for high-field magnets" (PDF). Applied Superconductivity 10 (1): 975-978, 2000. 
[189] Matthias, B. T.; Geballe, T. H.; Geller, S.; Corenzwit, E., "Superconductivity of Nb3Sn," Physical Review 95 (6): 1435-1435, 1954.

[190] Geballe, Theodore H., "Superconductivity: From Physics to Technology," Physics Today 46 (10): 52-56, October 1993.

[191] Godeke, A., "A review of the properties of Nb3Sn and their variation with A15 composition, morphology and strain state," Supercond. Sci. Technol. 19 (8): R68-R80, 2006.

[192] "Results of the first tests on the ITER toroidal magnet conductor," Commissariat à l'Énergie Atomique. 2001-09-10. Retrieved 2008-09-06.

[193] G. Grunblatt; P. Mocaer, Ch. Verwaerde and C. Kohler, "A success story: LHC cable production at ALSTOM-MSA," Fusion Engineering and Design (Proceedings of the 23rd Symposium of Fusion Technology). 75-79: 1-5, 2005.

[194] "Alstom and Oxford Instruments Team Up to Offer Niobium-Tin Superconducting Strand," Alstrom. 2007-06-27. Retrieved 2008-09-06.

[195] Flükiger, R.; Hariharan, S. Y.; Küntzler, R.; Luo, H. L.; Weiss, F.; Wolf, T.; Xu, J.Q., Flükiger, R.; Klose, W., eds. Nb-Ti. 21b2: Nb-H - Nb-Zr, Nd - Np (The Landolt-Börnstein Database ed.). SpringerMaterials, 1994.

[196] T.G. Berlincourt and R.R. Hake, "Pulsed-Magnetic-Field Studies of Superconducting Transition Metal Alloys at High and Low Current Densities," Bull. Am. Phys. Soc. II 7 408, 1962.

[197] T.G. Berlincourt, "Emergence of Nb-Ti as Supermagnet Material," Cryogenics 27, 283, 1987.

[198] "Conectus - Market", Conectus.org, 2015. [Online]. Available: http://www.conectus.org/xxmarket.html. [Accessed: 14- Jul- 2015].

[199] B.B. Goodman Rep. Prog. Phys. 39 (2), pp. 445, 1966.

[200] P.J. Lee et al, Chapter 5 in "Composite Superconductors" ed. K. Osamura, pub Marcel Dekker 1994.

[201] P.J. Lee, D.C. Larbalestier, J.C. McKinnell, McInturff AD: IEEE Trans Appl Supercon. Vol 3 No. 1, pp. 1354, 1993.

[202] D.C. Larbalestier, P.E. Madsen, J. Lee, M.N. Wilson, J.P. Charlesworth, IEEE Trans MAG-11, pp. 247, 1974. 
[203] N. Higuchi, K. Tsuchiya, C.J. Klamut, M. Suenaga, Adv Cryo Eng Vol 30, pp. $739,1984$.

[204] E.M. Hornsveld, J.D. Elen, C.A.M Van Beijnen, P. Hoogendam, Adv Cryo Eng Vol 34, pp. 493, 1987.

[205] J.W. Ekin, Adv Cryo Eng Vol 24, pp. 306, 1978.

[206] R. Flukiger, R. Isernhgen, W. Goldacker, W. Specking, Adv Cryo Eng Vol 30, pp. 851, 1984.

[207] Y. Iijima, M. Kosuge, T. Takeuchi, K. Inoue, Adv Cryo Eng Vol 40, pp. 899, 1994.

[208] Z.J.J. Stekly, J.L. Zar, IEEE Trans NS 12, pp. 367, 1965.

[209] T.Akachi, T. Ogasawara, K. Yasukochi, Jap Jnl App Phys 20 (8), pp. 1559, 1981.

[210] Superconducting Applications Group, Rutherford Laboratory, Brit Jnl Phys D (App Phys) Vol 3, pp. 1517, 1970.

[211] Wilson MN, Superconducting Magnets, pub Oxford University Press ISBN 0019- 854805-2.

[212] S.L. Wipf and A.P. Martinelli, Proc 1972 Appl. Supercon. Conf., pp. 331, Annapolis, 1972.

[213] A. Harada, Y. Mawatari, O. Miura, Y. Tanaka, K. Yamafuji, Cryogenics Vol 31, pp. 183, 1991.

[214] E. Gregory, H. Liu, J.M. Seuntjens, Adv Cryo Eng Vol 40, pp. 779, 1994.

[215] "YBCO", Ch.ic.ac.uk, 2015. [Online]. Available: http://www.ch.ic.ac.uk/rzepa/mim/century/html/ybco.htm. [Accessed: 14- Jul2015].

[216] "IBM Researchers reveal method for doubling superconductor Tc's", Superconductors.org, 2015.2 [Online]. Available: http://www.superconductors.org/doubleTc.htm. [Accessed: 14- Jul- 2015].

[217] "Superconductors See the Light at Shorter Wavelengths", Superconductors.org, 2015. [Online]. Available: http://www.superconductors.org/lightdet.htm. [Accessed: 14- Jul- 2015]. 
[218] Cartlidge, Edwin, "Superconductivity record sparks wave of follow-up physics," Nature News. Retrieved 18 August 2015.

[219] Dai, P.; Chakoumakos, B.C.; Sun, G.F.; Wong, K.W.; Xin, Y.; Lu, D.F., "Synthesis and neutron powder diffraction study of the superconductor HgBa2Ca2Cu3O8+ $\delta$ by Tl substitution," Physica C 243 (3-4): 201-206, 1995.

[220] Prins, JF, "The diamond-vacuum interface: II. Electron extraction from n-type diamond: evidence for superconduction at room temperature," Semiconductor Science and Technology 18 (3): S131, 2003.

[221] Tripodi, P.; Di Gioacchino, D.; Borelli, R.; Vinko, J. D., "Possibility of high temperature superconducting phases in PdH," Physica C: Superconductivity. 388-389: 571-572, May 2003.

[222] Tripodi, P.; Di Gioacchino, D.; Vinko, J. D., "Superconductivity in PdH: Phenomenological explanation," Physica C: Superconductivity. 408-410: 350352, August 2004.

[223] Tripodi, P.; Di Gioacchino, D.; Vinko, J. D., "A review of high temperature superconducting property of PdH system," International Journal of Modern Physics B 21 (18\&19): 3343-3347, 2007.

[224] Scheike, T.; Böhlmann, W.; Esquinazi, P.; Barzola-Quiquia, J.; Ballestar, A.; Setzer, A., "Can Doping Graphite Trigger Room Temperature Superconductivity? Evidence for Granular High-Temperature Superconductivity in Water-Treated Graphite Powder," Advanced Materials 24 (43): 5826, 2012.

[225] Mankowsky, R.; Subedi, A.; Först, M.; Mariager, S. O.; Chollet, M.; Lemke, H. T.; Robinson, J. S.; Glownia, J. M.; Minitti, M. P.; Frano, A.; Fechner, M.; Spaldin, N. A.; Loew, T.; Keimer, B.; Georges, A.; Cavalleri, A., "Nonlinear lattice dynamics as a basis for enhanced superconductivity in $\mathrm{YBa} 2 \mathrm{Cu} 3 \mathrm{O} 6.5$," Nature 516 (7529): 71-73, 2014.

[226] Ge, Y. F.; Zhang, F.; Yao, Y. G., "First-principles demonstration of superconductivity at $280 \mathrm{~K}$ in hydrogen sulfide with low phosphorus substitution," Phys. Rev. B 93 (22): 224513, 2016.

[227] Ashcroft, N. W., "Metallic Hydrogen: A High-Temperature Superconductor?," Physical Review Letters 21 (26): 1748-1749, 1968.

[228] Little, W. A., "Possibility of Synthesizing an Organic Superconductor," Physical Review 134 (6A): A1416-A1424, 1964. 
[229] Schilling, A.; Cantoni, M.; Guo, J. D.; Ott, H. R, "Superconductivity in the HgBa-Ca-Cu-O system". Nature 363 (6424): 56-58, 1993.

[230] L. Henley, "Lecture 6.7 Flux-flow conductivity." [Online]. Available: http://people.ccmr.cornell.edu/ clh/BT-GL/6.7.pdf.

[231] M. Tinkham, "Introduction to Superconductivity," Second edition. Dover Publications, 2004.

[232] J. W. Guikema, H. Bluhm, D. A. Bonn, R. Liang, W. N. Hardy, and K. A. Moler, "Two-dimensional vortex behavior in highly underdoped YBa2Cu3O6+x observed by scanning Hall probe microscopy," Phys. Rev. B, vol. 77, no. 10, p. 104515, 2008.

[233] A. N. Grigorenko, S. J. Bending, A. E. Koshelev, J. R. Clem, T. Tamegai, and S. Ooi, "Visualization of Interacting Crossing Vortex Lattices in the Presence of Quenched," Phys. Rev. Lett. 89, 217003, 2002.

[234] M. Beleggia, G. Pozzi, A. Tonomura, H. Kasai, T. Matsuda, K. Harada, T. Akashi, T. Masui, and S. Tajima, "Model of Superconducting Votices in Layered Materials for The Interpretation of Transmission Electron Microscopy Images," Phys. Rev. B 70, 184518, 2004.

[235] M. Benkraouda and J. R. Clem, "Magnetic Hysteresis from the Geometrical Barrier in Type-II Superconducting Strips," Phys. Rev. B 53, 438, 1996.

[236] J. R. Kirtley, P. Chaudhari, M. B. Ketchen, N. Khare, S.-Y. Lin, and T. Shaw, "Fractional Vortices as Evidence of Time-Reversal Symmetry in HighTemperature Superconductors," Phys. Rev. B 51, 12057, 1995.

[237] J. Mannhart, H. Hilgenkamp, B. Mayer, C. Gerber, J. R. Kirtley, K. A. Moler, and M. Sigrist, Phys. Rev. Lett. 77, 2782, 1996.

[238] R. Liang, D. A. Bonn, and W. N. Hardy, "Limits on Spin-Charge Seperation from $h / 2 e$ Fluxoid in Very Underdoped $\mathrm{YBa}_{2} \mathrm{Cu}_{3} \mathrm{O}_{6+x}$," Physica (Amsterdam) 304C, 105, 1998.

[239] R. Liang, D. A. Bonn, W. N. Hardy, J. C. Wynn, K. A. Moler, L. Lu, S. Larochelle, L. Zhou, M. Greven, L. Lurio, et al., "Preparation and Characterization of Homogeneous YBCO Single Crystals with Doping Level Near The SC-AFM Boundary," Physica (Amsterdam) 383C, 1, 2002.

[240] J. W. Guikema, Ph.D. Thesis, "Scanning Hall Probe Microscopy of Magnetic Vortices in Very Underdoped Yttrium-Barium-Copper-Oxide," Ph.D. Thesis Stanford University, 2004. 
[241] A. M. Chang, H. D. Hallen, L. Harriott, H. F. Hess, H. L. Kao, J. Kwo, R. E. Miller, R. Wolfe, J. van der Ziel, and T. Y. Chang, "Scanning Hall Probe Microscopy," Appl. Phys. Lett. 61, 1974, 1992.

[242] D. Davidovi'c, S. Kumar, D. H. Reich, J. Siegel, S. B. Field, R. C. Tiberio, R. Hey, and K. Ploog, "Correlations and disorder in arrays of magnetically coupled superconducting rings," Phys. Rev. Lett. 76, 815, 1996.

[243] A. Oral, S. J. Bending, and M. Henini, "Real-time scanning hall probe microscopy," J. Vac. Sci. Technol. B 14, 1202, 1996.

[244] G. J. Dolan, F. Holtzberg, C. Feild, and T. R. Dinger, "Anisotropic vortex structure in $\mathrm{Y}_{1} \mathrm{Ba}_{2} \mathrm{Cu}_{3} \mathrm{O}_{7}$," Phys. Rev. Lett. 62, 2184, 1989.

[245] A. Hosseini, D. M. Broun, D. E. Sheehy, T. P. Davis, M. Franz, W. N. Hardy, R. Liang, and D. A. Bonn, "Survival of the d-wave superconducting state near the edge of antiferromagnetism in the cuprate phase diagram," Phys. Rev. Lett. 93, 107003, 2004.

[246] R. Liang, D. A. Bonn, W. N. Hardy, and D. Broun, "Lower critical field and superfluid density of highly underdoped $\mathrm{YBa}_{2} \mathrm{Cu}_{3} \mathrm{O}_{6+x}$," Phys. Rev. Lett. 94, 117001, 2005.

[247] K. E. Gray, D. H. Kim, B. W. Veal, G. T. Seidler, T. F. Rosenbaum, and D. E. Farrell, "High anisotropy and a dimensionality crossover in the irreversibility behavior of oxygen-deficient $\mathrm{YBa}_{2} \mathrm{Cu}_{3} \mathrm{O}_{7-y}$," Phys. Rev. B 45, 10071, 1992.

[248] J. R. Clem,"2D pancake vortices in a finite stack of superconducting layers," Physica (Amsterdam) 235-240C, 2607, 1994,

[249] R. G. Mints, V. G. Kogan, and J. R. Clem, "Vortices in magnetically coupled superconducting layered systems," Phys. Rev. B 61, 1623, 2000.

[250] A. N. Grigorenko, S. J. Bending, G. D. Howells, and R. G. Humphreys, "Fluxline interractions with precipitates in $\mathrm{YBa} 2 \mathrm{Cu} 3 \mathrm{O} 7-\mathrm{x}$ films revealed by scanning hall probe microscopy," Phys. Rev. B 62, 721, 2000.

[251] B. W. Gardner, J. C. Wynn, D. A. Bonn, R. Liang, W. N. Hardy, J. R. Kirtley, V. G. Kogan, and K. A. Moler, "Manipulation of single vortices in $\mathrm{YBa}_{2} \mathrm{Cu}_{3} \mathrm{O}_{6.354}$ with locally applied magnetic field," Appl. Phys. Lett. 80, 1010, 2002.

[252] D. N. Basov, R. Liang, D. A. Bonn, W. N. Hardy, B. Dabrowski, M. Quijada, D. B. Tanner, J. P. Rice, D. M. Ginsberg, and T. Timusk, "In-plane anisotropy of the penetration depth in $\mathrm{YBa}_{2} \mathrm{Cu}_{3} \mathrm{O}_{7-x}$ and $\mathrm{YBa}_{2} \mathrm{Cu}_{4} \mathrm{O}_{8}$ superconductors," Phys. Rev. Lett. 74, 598, 1995. 
[253] A. E. Koshelev, "Vortex-chain phases in layered superconductors," Phys. Rev. B 71, 174507, 2005.

[254] L. N. Bulaevskii, M. Ledvij, and V. G. Kogan, "Vortices in layered superconductors with Josephson coupling," Phys. Rev. B 46, 366, 1992.

[255] A. Grigorenko, S. Bending, T. Tamegai, S. Ooi, and M. Henini, "A Onedimensional chain state of vortex matter," Nature (London) 414, 728, 2001.

[256] A. E. Koshelev, "Crossing lattices, vortex chains, and angular dependence of melting line in layered superconductors," Phys. Rev. Lett. 83, 187, 1999.

[257] Y. J. Uemura, G. M. Luke, B. J. Sternlieb, J. H. Brewer, J. F. Carolan, W. N. Hardy, R. Kadono, J. R. Kempton, R. F. Kiefl, S. R. Kreitzman, et al., Universal correlations between $T_{c}$ and $n_{s} / m^{*}$ (carrier density over effective masss) in High$\mathrm{T}_{\mathrm{c}}$ cuprate superconductors," Phys. Rev. Lett. 62, 2317, 1989.

[258] Y. J. Uemura, L. P. Le, G. M. Luke, B. J. Sternlieb, W. D. Wu, J. H. Brewer, T. M. Riseman, C. L. Seaman, M. B. Maple, M. Ishikawa, et al., "Basic similarities among cuprate, bismuthate, organic, chevrel-phase, and heavy-fermion superconductors shown by penetration-depth measurements," Phys. Rev. Lett. 66, 2665, 1991.

[259] J. Pearl, "Structure of superconductive vortices near a metal-air interface," $J$. Appl. Phys. 37, 4139, 1966.

[260] V. G. Kogan, A. Y. Simonov, and M. Ledvij, "Magnetic field of vortices crossing a superconductor surface," Phys. Rev. B 48, 392, 1993.

[261] J. R. Kirtley, V. G. Kogan, J. R. Clem, and K. A. Moler, "Magnetic field of an in-plane vortex outside a layered superconductor," Phys. Rev. B 59, 4343, 1999.

[262] J. R. Kirtley, C. C. Tsuei, K. A. Moler, V. G. Kogan, J. R. Clem, and A. J. Turberfield, "Variable sample temperature scanning superconducting quantum interference device microscope," Appl. Phys. Lett. 74, 4011, 1999.

[263] T. Pereg-Barnea, P. J. Turner, R. Harris, G. K. Mullins, J. S. Bobowski, M. Raudsepp, R. Liang, D. A. Bonn, and W. N. Hardy, "Absolute values of the London penetration depth in $\mathrm{YBa} 2 \mathrm{Cu} 3 \mathrm{O} 6+\mathrm{y}$ measured by zero field ESR spectroscopy on Gd doped single crytals," Phys. Rev. B 69, 184513, 2004.

[264] Y. Zuev, M. S. Kim, and T. R. Lemberger, "Correlation between superfluid density and $\mathrm{Tc}$ of underdoped $\mathrm{YBa}_{2} \mathrm{Cu}_{3} \mathrm{O}_{6+x}$ near the superconductor-insulator transition," Phys. Rev. Lett. 95, 137002, 2005. 
[265] L. F. Herbut and M. J. Case, "Finite-temperature superfluid density in very underdoped cuprates," Phys. Rev. B 70, 094516, 2004.

[266] J. R. Clem, "Two-dimensional vortices in a stack of thin superconducting films: A model for high-temperature superconducting multilayers," Phys. Rev. B, vol. 43, no. 10, pp. 7837-7846, 1991

[267] T. Pe, M. Benkraouda, and J. R. Clem, "Magnetic coupling of two-dimensional pancake vortex lattices in a finite stack of thin superconducting films with transport currents in the two outermost layers," Phys. Rev. B, vol. 55, no. 10, pp. 6636-6636, 1997.

[268] J. R. Clem, "Magnetic Flux Penetration into the High-Temperature Superconductors," in Physics and Materials Science of Vortex States, Flux Pinning and Dynamics, R. Kossowsky, Ed. Kluwer Academic Publishers, pp. 57-80. 1999.

[269] J. R. Clem, "Pancake Vortices," J. Supercond. Inc. Nov. Magn., vol. 17, pp. 613-630, 2004.

[270] S. J. Bending and M. J. W. Dodgson, "Vortex chains in anisotropic superconductors," J. Phys. Condens. Matter, vol. 17, no. 35, p. R955, 2005.

[271] D. Milliken, T. Silver, and S. X. Dou, "Irradiation of HTS for Enhancement of Critical Current," in Frontiers in Superconducting Materials, A. V. Narlikar, Ed. Berlin Heidelberg: Springer, pp. 555-588, 2005.

[272] B. M. Kessler, Ç. Ö. Girit, A. Zettl, and V. Bouchiat, "Tunable Superconducting Phase Transition in Metal-Decorated Graphene Sheets," Phys. Rev. Lett., vol. 104, no. 4, p. 047001, 2010.

[273] I. L. Spain, A. R. Ubbelohde, and D. A. Young, "Electronic Properties of Well Oriented Graphite," Philos. Trans. R. Soc. Lond. Math. Phys. Eng. Sci., vol. 262, no. 1128, pp. 345-386, 1967.

[274] I. L. Spain, "The Electronic Properties of Graphite," Chem. Phys. Carbon, vol. 8, pp. 1-150, 1973.

[275] Krishnan, K. S., "Magnetic Anisotropy of Graphite," Nature, vol. 133, no. 3353, pp. 174-175, 1934.

[276] Goetz, Alexandder; Focke, Alfred B.; Faessler, Alfred, "The Production of Large Artificial Graphite Crystals," [Online] Available: http://adsabs.harvard.edu/cgi-bin/nph- 
data_query?bibcode $=1932 \mathrm{PhR}$...39..168G\&db_key=PHY\&link_type=ABSTR ACT

[277] G. Berdiyorov, "Vortex Structure and Critical Parameters in Superconducting Thin Films with Arrays of Pinning Centers," PhD Dissertation, University of Antwerpen, Antwerpen, Belguim, 2007.

[278] A. K. Geim, S. V. Dubonos, I. V. Grigorieva, K. S. Novoselov, F. M. Peeters, and V. A. Schweigert, "Non-quantized penetration of magnetic field in the vortex state of superconductors," Nature, vol. 407, no. 6800, pp. 55-57, 2000.

[279] J. Bardeen, "Quantization of Flux in a Superconducting Cylinder," $A A$ (University of Illinois, Urbana, Illinois), Physical Review Letters, vol. 7, Issue 5, pp. 162-163, 09/1961.

[280] Fetter, Alexander L., "Flux penetration in a thin superconducting disk," [Online]. Available: http://adsabs.harvard.edu/cgi-bin/nphdata_query?bibcode $=1980 \mathrm{PhRvB} . .22 .1200 \mathrm{~F} \& d b \_k e y=$ PHY\&link_type=ABST RACT

[281] Fuchs, Dan T.; Zeldov, Eli; $\quad$ Rappaport, Michael; $\quad$ Tamegai, Tsuyoshi; Ooi, Shuuichi; Shtrikman, Hadas, "Transport properties governed by surface barriers in $\mathrm{Bi}_{2} \mathrm{Sr}_{2} \mathrm{CaCu}_{2} \mathrm{O}_{8}$," [Online]. Available: http://adsabs.harvard.edu/cgi$\mathrm{bin} / \mathrm{nph}$ -

data_query?bibcode=1998Natur.391..373F\&db_key=PHY\&link_type=ABSTR ACT

[282] Geim, A. K.; Dubonos, S. V.; Lok, J. G. S.; Grigorieva, I. V.; Maan, J. C.; Hansen, L. Theil; Lindelof, P. E., "Ballistic Hall Micro-Magnetometry," [Online]. Available: http://adsabs.harvard.edu/cgi-bin/nphdata_query?bibcode=1997ApPhL..71.2379G\&db_key=PHY\&link_type=ABST RACT

[283] Geim, A. K.; $\quad$ Dubonos, S. V.; $\quad$ Lok, J. G. S.; $\quad$ Henini, M.; $\quad$ Maan, J. C., "Paramagnetic Meissner effect in small superconductors," [Online]. Available: http://adsabs.harvard.edu/cgi-bin/nph-

data_query?bibcode=1998Natur.396..144G\&db_key=PHY\&link_type=ABSTR ACT

[284] Geim, A. K.; Dubonos, S. V.; Palacios, J. J.; Grigorieva, I. V.; Henini, M.; Schermer, J. J., "Fine Structure in Magnetization of Individual Fluxoid States," [Online] Available: http://adsabs.harvard.edu/cgi-bin/nphdata_query?bibcode $=2000 \mathrm{PhRvL} . .85 .1528 \mathrm{G} \& d b \_k e y=$ PHY\&link_type=ABST RACT 
[285] Kogan, Vladimir G., "Pearl's vortex near the film edge," [Online] Available: http://adsabs.harvard.edu/cgi-bin/nph-

data_query?bibcode $=1994 \mathrm{PhRvB} . .4915874 \mathrm{~K} \& d b \_k e y=P H Y \& l i n k \_t y p e=A B S T$ RACT

[286] Palacios, J. J., "Metastability and Paramagnetism in Superconducting Mesoscopic Disks," [Online] Available: http://adsabs.harvard.edu/cgi-bin/nphdata_query?bibcode $=2000$ PhRvL..84.1796P \&db_key=PHY\&link_type=ABST RACT

[287] White, W. R.; Kapitulnik, A.; Beasley, M. R., "Collective vortex motion in aMoGe superconducting thin films," [Online] Available: http://adsabs.harvard.edu/cgi-bin/nphdata_query?bibcode $=1993 \mathrm{PhRvL} . .70 . .670 \mathrm{~W} \& d b \_k e y=P H Y \& l i n k \_t y p e=A B S T$ RACT

[288] G. Profeta, M. Calandra, and F. Mauri, "Phonon-mediated superconductivity in graphene by lithium deposition," Nat. Phys., vol. 8, no. 2, pp. 131-134, 2012.

[289] K.S. Novoselov, D. Jiang, T. Booth, V.V. Khotkevich, S.M. Morozov, A.K. Geim, "Two Dimensional Atomic Crystal," Proc. Nat. Acad. Sci. 102, 10451, 2005.

[290] Geim, A. K., "Graphene: Status and prospects,” Science 324, pp. 15301534, 2009.

[291] Katsnelson, M. I., Novoselov, K. S. \& Geim, A. K. "Chiral Tunneling and The Klein Paradox in Graphene,” Nature Phys. 2, pp. 620-625, 2006.

[292] Novoselov, K. S. et al., "Room-temperature quantum Hall effect in graphene," Science 315,1379, 2007.

[293] Zhang, Y., Tan, Y. W., Stormer, H. L. \& Kim, P. "Experimental observation of the quantum Hall effect and Berry's phase in graphene," Nature 438, pp. 201204, 2005.

[294] Nair, R. R. et al. "Fine structure constant defines visual transparency of graphene," Science 320, 1308, 2008.

[295] De Franceschi, S., Kouwenhoven, L., Schönenberger, Ch. \& Wernsdorfer, W., "Hybrid superconductor-quantum dot devices," Nature Nanotech. 5, 703711, 2010.

[296] Huefner, M. et al. "Scanning gate microscopy measurements on a superconducting single-electron transistor," Phys. Rev. B 79, 134530, 2009. 
[297] Delahaye, J. et al. "Low-noise current amplifier based on mesoscopic Josephson junction," Science 299, pp. 1045-1048, 2003.

[298] Olli-Pentti, S. et al. "Heat-transistor: Demonstration of gate-controlled electronic refrigeration," Phys. Rev. Lett. 99, 027203, 2007.

[299] Piscanec, S. et al. "Kohn anomalies and electron-phonon interactions in graphite," Phys. Rev. Lett. 93, 185503, 2004.

[300] Csányi, G. et al. "The role of the interlayer state in the electronic structure of superconducting graphite intercalated compounds," Nature Phys. 1, pp. 42-45, 2005.

[301] Calandra, M. \& Mauri, F. "Possibility of Superconductivity in Graphite Intercalated with Alkaline Earths Investigated with Density Functional Theory," Phys. Rev. B 74, 094507, 2006.

[302] Kim, J. S. et al. "Superconductivity in Heavy Alkaline-Earth Intercalated Graphites," Phys. Rev. Lett. 99, 027001, 2007.

[303] Gauzzi, A. et al. "Enhancement of superconductivity and evidence of structural instability in intercalated graphite $\mathrm{CaC}_{6}$ under high pressure," Phys. Rev. Lett. 98, 067002, 2007.

[304] Giannozzi, P. et al. "QUANTUM ESPRESSO: A modular and open-source software project for quantum simulations of material," J. Phys. Condens. Matter 21, 395502, 2009.

[305] Appendix C of J. P. Perdew and A. Zunger, "Self-interaction correction to density-functional approximations for many-electron systems," Phys. Rev. B 23, pp. 5048-5079, 1981.

[306] Calandra, M. \& Mauri, F. "Theoretical explanation of superconductivity in $\mathrm{C}_{6}$ Ca," Phys. Rev. Lett. 95, 237002, 2005.

[307] Boeri, L. et al. "Electron-phonon interaction in graphite intercalation compounds," Phys. Rev. B 76, 064510, 2007.

[308] Guerarda, D. \& Herold, A. "Intercalation of lithium into graphite and other carbons," Carbon, 13, pp. 337-345, 1975.

[309] Caragiu, M. \& Finberg, S. "Alkali metal adsorption on graphite: A review," J. Phys. Condens. Matter 17, R995-R1024, 2005. 
[310] Loktev, V. M. \& Turkowski, V. "Possible high-temperature superconductivity in multilayer graphane: Can the cuprates be beaten?" J. Low Temp. Phys. 164, pp. 264-271, 2011.

[311] R. Nandkishore, L. S. Levitov, and A. V. Chubukov, "Chiral superconductivity from repulsive interactions in doped graphene," Nat. Phys., vol. 8, no. 2, pp. 158-163, 2012.

[312] DasSarma, S., Adam, S., Hwang, E. H. \& Rossi, E.," Electronic transport in two-dimensional graphene," Rev. Mod. Phys. 83, pp. 407-470, 2011.

[313] Guinea, F. "Viewpoint: Peeling back the layers or doubling the stakes? Calculations of bilayer graphene reveal the possibility of new electronic phases," Physics, 3, 1, 2010.

[314] Gonzalez, J. "Kohn-Luttinger superconductivity in graphene," Phys. Rev. B 78, 205431, 2008.

[315] Valenzuela, B. \& Vozmediano, M. A. H. "Pomeranchuk instability in doped graphene," New. J. Phys. 10, 113009, 2008.

[316] Martin, I. \& Batista, C. D. "Itinerant electron-driven chiral magnetic ordering and spontaneous quantum Hall effect in triangular lattice models," Phys. Rev. Lett. 101, 156402, 2008.

[317] Li, T. "Spontaneous quantum Hall effect in quarter doped Hubbard model on honeycomb lattice and its possible realization in quarter doped graphene system," [Online]. Available: http://arxiv.org/abs/1103.2420, 2011.

[318] Makogon, D., van Gelderen, R., Roldan, R. \& Morais Smith, C. "Spin-densitywave instability in graphene doped near the van Hove singularity," Phys. Rev. B 84, 125404, 2011.

[319] Volovik, G. E. "Quantized hall effect in superfluid helium-3 film," Phys. Lett. A 128, pp. 277-279, 1988.

[320] Sigrist, M. \& Ueda, K. "Phenomenological theory of unconventional superconductivity," Rev. Mod. Phys. 63, pp. 239-311, 1991.

[321] Vojta, M., Zhang, Y.\& Sachdev, S. "Quantum phase transitions in $d$-wave superconductors," Phys. Rev. Lett. 85, pp. 4940-4943, 2000.

[322] Moore, G. \& Read, N. "Nonabelions in the fractional quantum hall effect," Nucl. Phys. B 360, pp.362-396, 1991. 
[323] Ivanov, D. A. "Non-Abelian statistics of half-quantum vortices in $p$-wave superconductors," Phys. Rev. Lett. 86, pp. 268-271, 2001.

[324] Fu, L. \& Kane, C. L. "Superconducting proximity effect and Majorana fermions at the surface of a topological insulator," Phys. Rev. Lett. 100, 096407, 2008.

[325] Qi， X. L., Hughes, T., Raghu, S. \& Zhang, S-C. “Time-reversal-invariant topological superconductors and superfluids in two and three dimensions," Phys. Rev. Lett. 102,187001, 2009.

[326] Cheng, M., Sun, K., Galitski, V. \& Das Sarma, S. "Stable topological superconductivity in a family of two-dimensional fermion models," Phys. Rev. B 81, 024504, 2010.

[327] Kopnin, N. B. \& Salomaa, M. M. "Mutual friction in superfluid ${ }^{3} \mathrm{He}$ : Effects of bound states in the vortex core," Phys. Rev. B 44, pp. 9667-9677, 1991.

[328] Volovik, G. E. \& Yakovenko, V. M. "Fractional charge, spin and statistics of solitons in superfluid ${ }^{3} \mathrm{He}$ film," J. Phys. Condens. Matter 1, pp. 5263-5274, 1989.

[329] Laughlin, R. B. "Magnetic induction of $d_{x-y}^{2}{ }^{2}+i d_{x y}$ order in high $T_{\mathrm{c}}$ superconductors," Phys. Rev. Lett. 80, pp. 5188-5191, 1998.

[330] Volovik, G. E. "On edge states in superconductors with time inversion symmetry breaking,” J. Exp. Theor. Phys. Lett. 66, pp. 522-527, 1997.

[331] Senthil, T., Marston, J. B. \& Fisher, M. P. A. "Spin quantum Hall effect in unconventional superconductors," Phys. Rev. B 60, pp. 4245-4254, 1999.

[332] Horovitz, B. \& Golub, A. "Superconductors with broken time-reversal symmetry: Spontaneous magnetization and quantum Hall effects," Phys. Rev. B 68, 214503, 2003.

[333] Sato, M., Takahashi, Y. \& Fujimoto, S. "Non-Abelian topological orders and Majorana fermions in spin-singlet superconductors," Phys. Rev. B 82, 134521, 2010.

[334] Mao, L., Shi, J., Niu, Q. \& Zhang, C. "Superconducting phase with a chiral $f$ wave pairing symmetry and Majorana fermions induced in a hole-doped semiconductor," Phys. Rev. Lett. 106, 157003, 2011.

[335] Mackenzie, A. P. \& Maeno, Y. "The superconductivity of $\mathrm{Sr}_{2} \mathrm{RuO}_{4}$ and the physics of spin-triplet pairing,” Rev. Mod. Phys. 75, pp. 657-712, 2003. 
[336] Uchoa, B. \& Castro Neto, A. H. "Superconducting states of pure and doped graphene," Phys. Rev. Lett. 98, 146801, 2007.

[337] Kohn, W. \& Luttinger, J. M. "New mechanism for superconductivity," Phys. Rev. Lett. 15, pp. 524-526, 1965.

[338] Schulz, H. J. "Superconductivity and antiferromagnetism in the twodimensional Hubbard model: Scaling theory," Europhys. Lett. 4, pp. 609-615, 1987.

[339] Dzyaloshinskii, I. E. "Maximal increase of the superconducting transition temperature due to the presence of van Hove singularities," Sov. Phys. JETP 66, pp. 848-854, 1987.

[340] Furukawa, N., Rice, T. M. \& Salmhofer, M. "Truncation of a two-dimensional Fermi surface due to quasiparticle gap formation at the saddle points," Phys. Rev. Lett. 81, pp. 3195-3198, 1998.

[341] McChesney, J. L. et al. "Extended van Hove singularity and superconducting instability in doped graphene," Phys. Rev. Lett. 104, 136803, 2010.

[342] Ye, J. T. et al. "Liquid-gated interface superconductivity on an atomically flat film," Nature Mater. 9, pp. 125-128, 2010.

[343] LeHur, K. \& Rice, T. M. "Superconductivity close to the Mott state: From condensed-matter systems to superfluidity in optical lattices," Ann. Phys. 324, pp. 1452-1515, 2009.

[344] Maiti, S. \& Chubukov, A. V. "Renormalization group flow, competing phases and the structure of superconducting gap in multiband models of iron-based superconductors," Phys. Rev. B 82, 214515, 2010.

[345] Thomale, R., Platt, C., Hanke, W. \& Bernevig, B. A. "Mechanism for explaining differences in the order parameters of FeAs-based and FeP-based pnictide superconductors," Phys. Rev. Lett. 106, 187003, 2011.

[346] Wallace, P. R. "The band theory of graphite," Phys. Rev. 71, pp. 622-634, 1947.

[347] Son, D. T. "Superconductivity by long-range colour magnetic interaction in high-density quark matter," Phys. Rev. D 59, 094019, 1999.

[348] Moon, E. G. \& Chubukov, A. V. "Quantum-critical pairing with varying exponents," J. Low Temp. Phys. 161, pp. 263-281, 2010. 
[349] Pathak, S., Shenoy, V. B. \& Baskaran, G. "Possible high-temperature superconducting state with a $d+i d$ pairing symmetry in doped graphene," Phys. Rev. B 81, 085431, 2010.

[350] Roy, B. \& Herbut, I. F. "Unconventional superconductivity on honeycomb lattice: Theory of Kekule order parameter," Phys. Rev. B 82, 035429, 2010.

[351] Mineev, V. P. \& Samokhin, K. V. "Introduction to Unconventional Superconductivity 69," Gordon and Breach Science Publishers, 1998.

[352] S. J. Hagen, C. J. Lobb, R. L. Greene, and M. Eddy, "Flux-flow Hall effect in superconducting T12Ba2CaCu2O8 films," Phys. Rev. B, vol. 43, no. 7, pp. 6246-6248, 1991.

[353] N. B. Kopnin, B. I. Ivlev, and V. A. Kalatsky, "The flux-flow hall effect in type II superconductors. An explanation of the sign reversal," J. Low Temp. Phys., vol. 90, no. 1-2, pp. 1-13, 1993.

[354] N. B. Kopnin and A. V. Lopatin, "Flux-flow Hall effect in clean type-II superconductors," Phys. Rev. B, vol. 51, no. 21, pp. 15291-15303, 1995.

[355] N. B. Kopnin, "Hall effect in moderately clean superconductors and the transverse force on a moving vortex," Phys. Rev. B, vol. 54, no. 13, pp. 94759483, 1996.

[356] S. D. Ha, R. Jaramillo, D. M. Silevitch, F. Schoofs, K. Kerman, J. D. Baniecki, and S. Ramanathan, "Hall effect measurements on epitaxial $\mathrm{SmNiO3}$ thin films and implications for antiferromagnetism," Phys. Rev. B, vol. 87, no. 12, p. 125150, 2013.

[357] H. Chen, Q. Niu, and A. H. MacDonald, "Anomalous Hall Effect Arising from Noncollinear Antiferromagnetism," Phys. Rev. Lett., vol. 112, no. 1, p. 017205, 2014.

[358] Pugh, E. M. \& Rostoker, N. "Hall effect in ferromagnetic materials," Rev. Mod. Phys. 25, pp. 151- 157, 1953.

[359] Nagaosa, N., Sinova, J., Onoda, S., MacDonald, A. H., \& Ong, N. P. “Anomalous Hall effect," Rev. Mod. Phys. 82, pp. 1539-1592, 2010.

[360] Karplus, R. \& Luttinger, J. M. "Hall effect in ferromagnetics," Phys. Rev. 95, pp. 1154-1160, 1954. 
[361] Sundaram, G. \& Niu, Q. "Wave-packet dynamics in slowly perturbed crystals: Gradient corrections and Berry-phase effects," Phys. Rev. B 59, pp. 1491514925, 1999.

[362] Chang, Cui-Zu, Zhang, Jinsong, Feng, Xiao, Shen, Jie, Zhang, Zuocheng, Guo, Minghua, Li, Kang, Ou, Yunbo, Wei, Pang, Wang, Li-Li, Ji, Zhong-Qing, Feng, Yang, Ji, Shuaihua, Chen, Xi, Jia, Jinfeng, Dai, Xi, Fang, Zhong, Zhang, ShouCheng, He, Ke, Wang, Yayu, Lu, Li, Ma, Xu-Cun, \& Xue, Qi-Kun, "Experimental observation of the quantum anomalous Hall effect in a magnetic topological insulator," Science 340, pp. 167-170, 2013.

[363] Chubukov, A. "Order from disorder in a kagomé antiferromagnet," Phys. Rev. Lett. 69, pp. 832- 835, 1992.

[364] Harris, A. B., Kallin, C., \& Berlinsky, A. J. "Possible Néel orderings of the Kagomé antiferromagnet," Phys. Rev. B 45, pp. 2899-2919, 1992.

[365] Huse, D. A. \& Rutenberg, A. D. "Classical antiferromagnets on the Kagomé lattice,” Phys. Rev. B 45, 12, pp. 7536-7539, 1992.

[366] Sachdev, S. "Kagomé- and triangular-lattice Heisenberg antiferromagnets: Ordering from quantum fluctuations and quantum-disordered ground states with unconfined bosonic spinons," Phys. Rev. B 45, pp. 12377-12396, 1992.

[367] Ritchey, I., Chandra, P., \& Coleman, P. "Spin folding in the two-dimensional Heisenberg kagomé antiferromagnet," Phys. Rev. B 47, pp. 15342-15345, 1993.

[368] Tomeno, I., Fuke, H. N., Iwasaki, H., Sahashi, M., \& Tsunoda, Y. "Magnetic neutron scattering study of ordered Mn3," J. Appl, Phys. 86, 3853, 1999.

[369] Hemmati, V., Plumer, M. L., \& Whitehead, J. P. "Monte Carlo simulations of magnetic ordering in the fcc kagome lattice," Phys. Rev. B 86, 104419, 2012. Ir. J. Appl. Phys. 86, pp. 3853-3856,1999.

[370] Szunyogh, L., Lazarovits, B., Udvardi, L., Jackson, J., \& Nowak, U. "Giant magnetic anisotropy of the bulk antiferromagnets IrMn and IrMn3," Phys. Rev. B 79, 020403(R), 2009.

[371] Herring, C. "in Magnetism: A Treatise on Modern Theory and Materials Vol. IV," edited by Rado, G. T. \& Suhl, H. (Academic Press, New York, 1966). from first principles. Phys. Rev. B 79, 020403(R), 2009.

[372] Xiao, D., Chang, M.-C., \& Niu, Q. "Berry phase effects on electronic properties," Rev. Mod. Phys. 82, pp. 1959-2007, 2010. 
[373] Ohgushi, K., Murakami, S., \& Nagaosa, N. "Spin anisotropy and quantum Hall effect in the kagomé lattice: Chiral spin state based on a ferromagnet," Phys. Rev. B 62, R6065-R6068, 2000.

[374] Kane, C. L. \& Mele, E. J. "Quantum spin Hall effect in graphene," Phys. Rev. Lett. 95, 226801, 2005.

[375] Tomizawa, T. \& Kontani, H. "Anomalous Hall effect in the t2g orbital kagome lattice due to noncollinearity: Significance of the orbital Aharonov-Bohm effect," Phys. Rev. B 80, 100401(R), 2009.

[376] Tomizawa, T. \& Kontani, H. "Anomalous Hall effect due to noncollinearity in pyrochlore compounds: Role of orbital Aharonov-Bohm effect," Phys. Rev. B 82, 100412, 2010.

[377] Yao, Y., Kleinman, L., MacDonald, A. H., Sinova, J., Jungwirth, T., Wang, D.S., Wang, E.- G., \& Niu, Q. "First principles calculation of anomalous Hall conductivity in ferromagnetic bcc Fe," Phys. Rev. Lett. 92, 037204, 2004.

[378] Krén, E., Kádár, G., Pál, L., Sólyom, J., Szabó, P., \& Tarnóczi, T. "Magnetic structures and exchange interactions in the Mn-Pt system," Phys. Rev. 171, pp. 574-585, 1968.

[379] Orenstein J. \& Moore, Joel E. "Berry phase mechanism for optical gyrotropy in stripe-ordered cuprates," Phys. Rev. B. 87, 165110, 2013.

[380] Perdew, J. P., Burke, K., \& Ernzerhof, M. "Generalized gradient approximation made simple,” Phys. Rev. Lett. 77, pp. 3865-3868, 1996.

[381] Yamaoka, T. “Antiferromagnetism in $\gamma$-phase Mn-Ir alloys," J. Phys. Soc. Jpn. 36, pp. 445-450, 1974.

[382] Monkhorst, H. J. \& Pack, J. D. "Special points for Brillouin-zone integrations," Phys. Rev. B 13, pp. 5188-5192, 1976.

[383] Sakuma, A., Fukamichi, K., Sasao, K., \& Umetsu, R. Y. "First-principles study of the magnetic structures of ordered and disordered Mn-Ir alloys," Phys. Rev. B 67, 024420, 2003.

[384] Wang, X., Yates, J. R., Souza, I., \& Vanderbilt, D. "Ab initio calculation of the anomalous Hall conductivity by Wannier interpolation," Phys. Rev. B 74, 195118, 2006. 
[385] Marzari, N. \& Vanderbilt, D. "Maximally localized generalized Wannier functions for composite energy bands," Phys. Rev. B 56, pp. 12847-12865, 1997.

[386] Mostofi, A. A., Yates, J. R., Lee, Y.-S., Souza, I., Vanderbilt, D., \& Marzari, N. "wannier90: A tool for obtaining maximally-localised Wannier functions. Comput," Phys. Commun. 178, pp. 685-699, 2008.

[387] Q. Shao, G. Liu, D. Teweldebrhan, and A. A. Balandin, "High-temperature quenching of electrical resistance in graphene interconnects," Appl. Phys. Lett., vol. 92, no. 20, p. 202108, 2008.

[388] A. Vassighi and M. Sachdev, "Thermal and Power Management of Integrated Circuits Springer," New York, Chap. 1, 2005.

[389] S. P. Gurrum, S. K. Suman, Y. K. Joshi, and A. G. Fedorov, "Thermal challenges in next-generation electronic systems," IEEE Trans. Device Mater Reliab. 4, 709, 2004; Y. Joshi, K. Azar, D. Blackburn, C. J. M. Lasance, R. Mahajan, and J. Rantala, Microelectron. J. 34, 1195, 2003.

[390] A. A. Balandin and K. L. Wang, "Significant decrease of the lattice thermal conductivity due to photon confinement in a free-standing semiconductor quantum well," Phys. Rev. B 58, 1544, 1998; A. A. Balandin, E. P. Pokatilov, and D. L. Nika, J. Nanoelectron. Optoelectron. 2, 140, 2007.

[391] E. P. Pokatilov, D. L. Nika, and A. A. Balandin, "Negative refraction: An intrinsic property of uniaxial crystals," Phys. Rev. B 72, 113311, 2005; J. Superlattices and Microstruct. 38, 168, 2005.

[392] N. Srivastava and K. Banerjee, "Proceedings of the 2005 IEEE/ACM International Conference on Computer-Aided Design IEEE," Washington DC, pp. 383-390, 2005.

[393] Q. Ngo, A. M. Cassell, A. J. Austin, J. Li, S. Krishnan, M. Meyyappan, and C. Y. Yang, "Characteristics of Aligned Carbon Nanofibers for Interconnect Via Applications," IEEE Electron Device Lett. 27, 221 2006; Q. Ngo, B. A. Cruden, A. M. Cassell, G. Sims, M. Meyyappan, J. Li, and C. Y. Yang, Nano Lett. 4, 2403, 2004.

[394] A. Naeemi and J. D. Meindl, "Compact physical models for multiwall carbonnanotube interconnects," IEEE Electron Device Lett. 28, 428, 2007.

[395] S. Hong, Y. Yoon, and J. Guo, "Metal-semiconductor junction of graphene nanoribbons," Appl. Phys. Lett. 92, 083107, 2008. 
[396] A. A. Balandin, S. Ghosh, W. Bao, I. Calizo, D. Teweldebrhan, F. Miao, and C. N. Lau, "Superior thermal conductivity of single-layer graphene," Nano Lett. 8 , 902, 2008.

[397] S. Ghosh, I. Calizo, D. Teweldebrhan, E. P. Pokatilov, D. L. Nika, A. A. Balandin, W. Bao, F. Miao, and C. N. Lau, "Extremely high thermal conductivity of graphene: Prospects for thermal management applications in nanoelectonic circuits," Appl. Phys. Lett. 92, 151911, 2008.

[398] G. Kuka, W. Kraak, H.-J. Gollnest, and R. Herrmann, "Temperature Dependence of the Resistivity in Semimetals of the Bismuth Type," Phys. Status Solidi B 89, 547, 1978.

[399] F. Parvizi, D. Teweldebrhan, S. Ghosh, I. Calizo, A. A. Balandin, H. Zhu, and R. Abbaschian, "Supior thermal conductivity of single-layer graphene," Micro \& Nano Lett. 3, 29, 2008.

[400] A. C. Ferrari, J. C. Meyer, V. Scardaci, C. Casiraghi, M. Lazzeri, F. Mauri, S. Piscanec, D. Jiang, K. S. Novoselov, S. Roth, and A. K. Geim, "Raman spectrum of graphene and graphene layers," Phys. Rev. Lett. 97, 187401, 2006.

[401] I. Calizo, F. Miao, W. Bao, C. N. Lau, and A. A. Balandin, "Graphene-onsapphire and graphene-on-glass: raman spectroscopy study," Appl. Phys. Lett. 91, 071913, 2007; I. Calizo, W. Bao, F. Miao, C. N. Lau, and A. A. Balandin, Appl. Phys. Lett. 91, 201904, 2007.

[402] I. Calizo, D. Teweldebrhan, W. Bao, F. Miao, C. N. Lau, and A. A. Balandin, "Temperature dependence of the raman spectra of graphene and graphene multilayer," J. Phys.: Condens. Matter 109, 012008, 2008; I. Calizo, A. A. Balandin, W. Bao, F. Miao, and C. N. Lau, Nano Lett. 7, 2645, 2007.

[403] G. Busch and H. Schade, "Lectures on Solid State Physics Pergamon," New York, p. 289, 1976.

[404] A. Seeger and K. Clausecker, "A new method for solving the Boltzmann equation for electrons in crystals," Phys. Status Solidi B46, 137, 1971.

[405] D. S. Choi, A. A. Balandin, M. S. Leung, G. Stupian, N. Presser, S. W. Chung, J. R. Heath, A. Khitun, and K. L. Wang, "Transport study of a single bismuth nanowire fabricated by the silver and silicon nanowire shadow masks," Appl. Phys. Lett. 89, 141503, 2006.

[406] F. T. Vasko and V. Ryzhii, "Voltage and temperature dependencies of conductivity in gated graphene," Phys. Rev. B 76, 233404, 2007. 
[407] V. V. Cheianov and V. I. Fal'ko, "Friedel oscillations, impurity scattering, and temperature dependence of resistivity in graphene," Phys. Rev. Lett. 97, 226801, 2006.

[408] S. Mrozowski, "Electric resistvity of polycrystalline graphite and carbons," Phys. Rev. 77, 838, 1950.

[409] J. M. Reynolds, H. W. Hemstreet, and T. E. Leinhardt, "The electrical resistance of graphite at low temperatures," Phys. Rev. 91, 1152, 1953.

[410] J. M. Skowronski, "Unusual temperature dependence of the electrical resistance of compacted $\mathrm{CrO} 3$ graphite intercalation compounds," Carbon 26, 613, 1988.

[411] Standard Handbook for Electrical Engineers McGraw-Hill, New York, pp. 484. 202108-3 Shao et al. Appl. Phys. 1993.

[412] K. I. Bolotin, K. J. Sikes, J. Hone, H. L. Stormer, and P. Kim, "TemperatureDependent Transport in Suspended Graphene," Phys. Rev. Lett., vol. 101, no. 9, p. 096802, 2008.

[413] E. Mariani and F. von Oppen, "Temperature-dependent resistivity of suspended raphene," Phys. Rev. B, vol. 82, no. 19, p. 195403, 2010.

[414] V. Skákalová, A. B. Kaiser, J. S. Yoo, D. Obergfell, and S. Roth, “Correlation between resistance fluctuations and temperature dependence of conductivity in graphene," Phys. Rev. B, vol. 80, no. 15, p. 153404, 2009.

[415] L. Weber, "Conductance Anisotropy in natural and HOPG Graphite," Master's thesis, University of Basel, 2010.

[416] Jobst and H. B. Weber, "Origin of logarithmic resistance correction in graphene," Nat. Phys., vol. 8, no. 5, pp. 352-352, 2012.

[417] Y.-W. Tan, Y. Zhang, H. L. Stormer, and P. Kim, "Temperature dependent electron transport in graphene," Eur. Phys. J. Spec. Top., vol. 148, no. 1, pp. 1518, 2007.

[418] F. Schedin, K.S. Novoselov, S.V. Morozov, D. Jiang, E.H. Hill, P. Blake, A.K. Geim, "Detection of individual gas molecules absorbed on graphene," condmat/0610834, 2007.

[419] Y. Tan, Y. Zhang, B. Oezyilmaz, K. Bolotin, Y. Zhao, H.L. Stormer, P. Kim "Experimental observation of the quantum hall effect and berry's phase in graphene," (unpublished) 
[420] C. Berger, Z. Song, T. Li, X. Li, A. Y. Ogbazghi, R. Feng, Z. Dai, A. N. Marchenkov, E. H. Conrad, P. N. First, and W. A. de Heer, "Ultrathin Epitaxial Graphite: 2D Electron Gas Properties and a Route toward Graphene-based Nanoelectronics," J. Phys. Chem. B, vol. 108, no. 52, pp. 19912-19916, 2004. 
APPENDICES 
Appendix $1 \quad R$ vs. $T$ for $\mathrm{YBa}_{2} \mathrm{Cu}_{3} \mathrm{O}_{7-\mathrm{x}}$ Target 


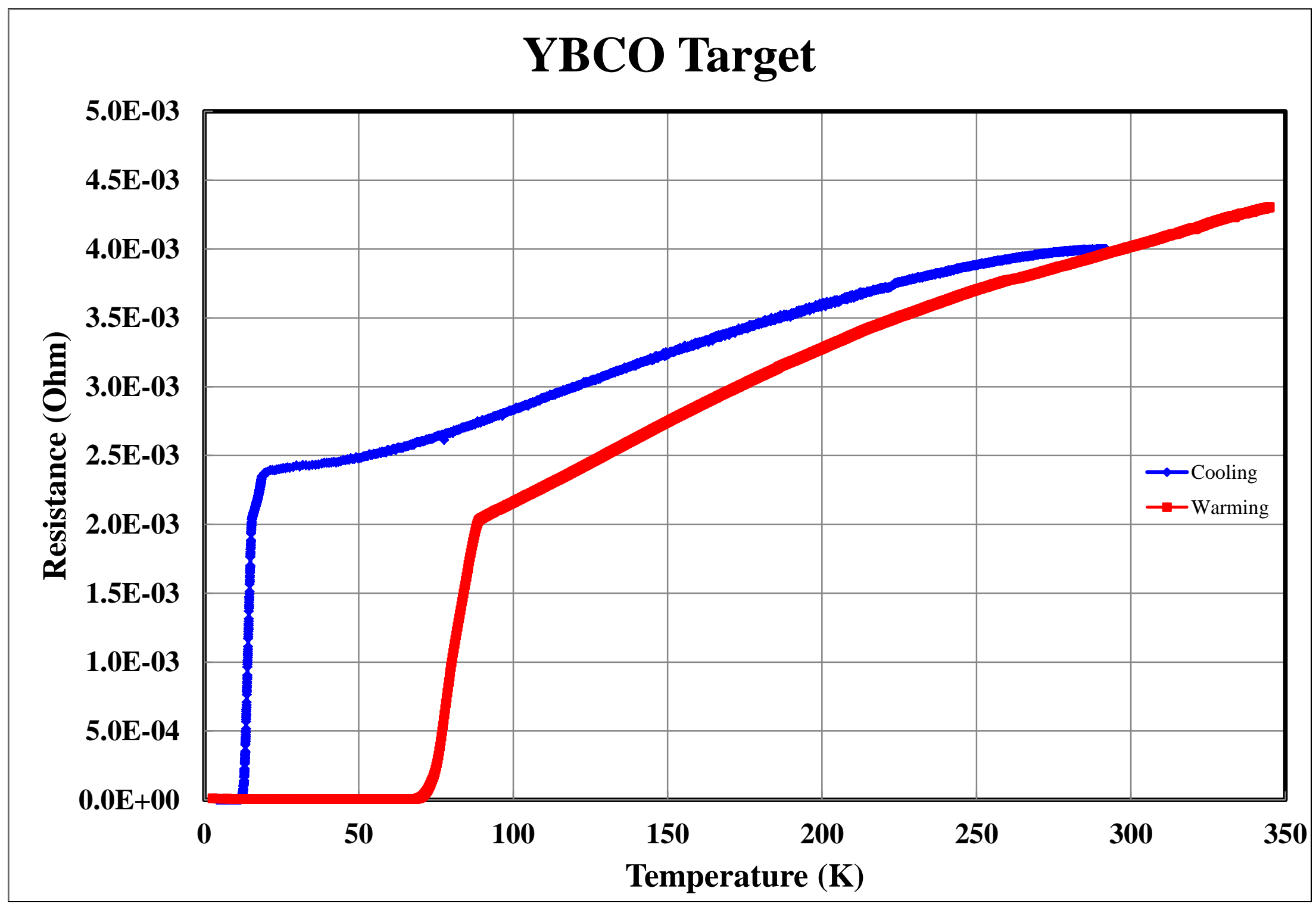


Appendix $2 \quad R$ vs. $T$ for $\mathrm{YBa}_{2} \mathrm{Cu}_{3} \mathrm{O}_{7-\mathrm{X}}$ Hairpin 


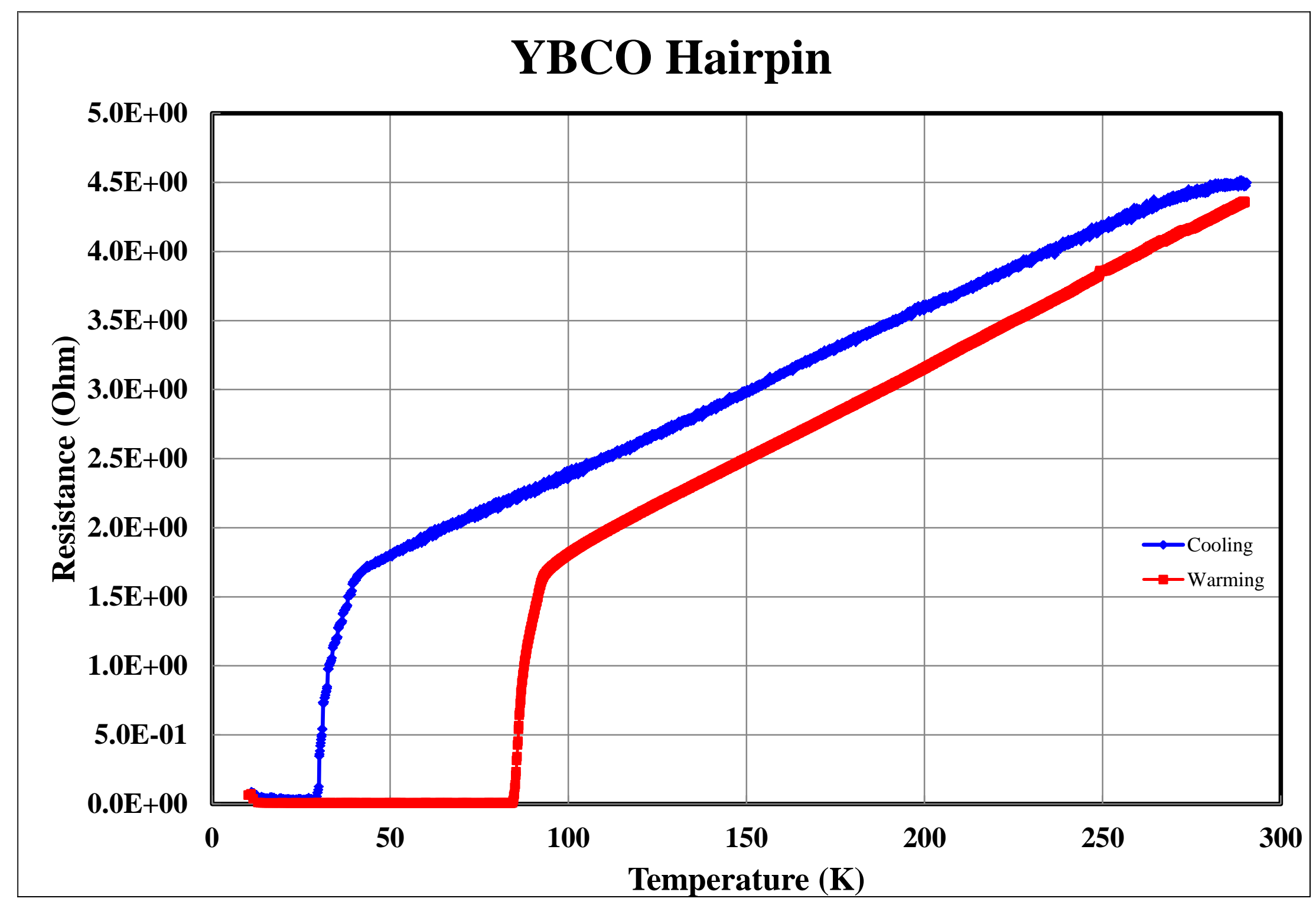




\section{Appendix $3 \quad R$ vs. $T$ for Niobium}




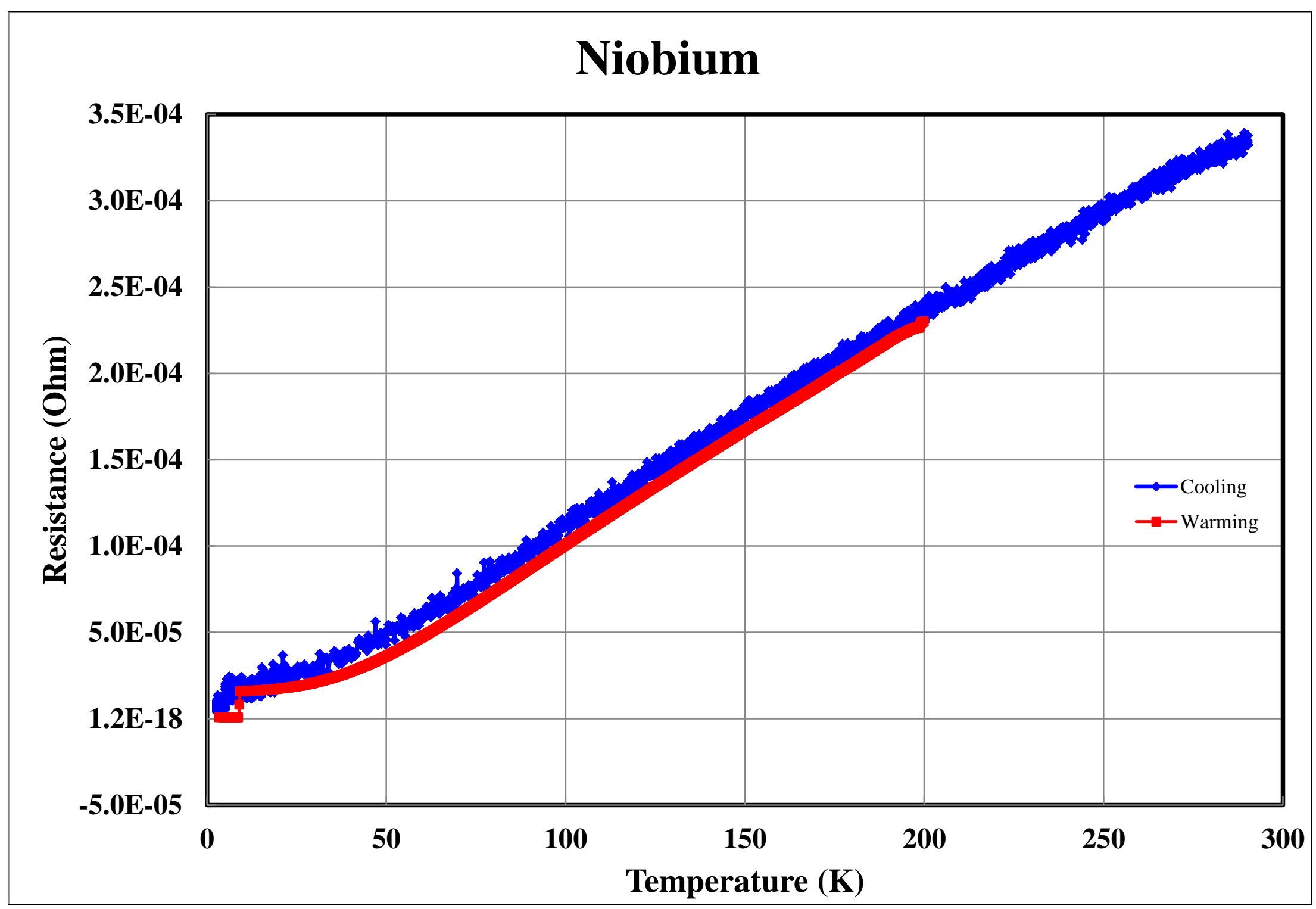


Appendix $4 \quad$ Loss of Contact 


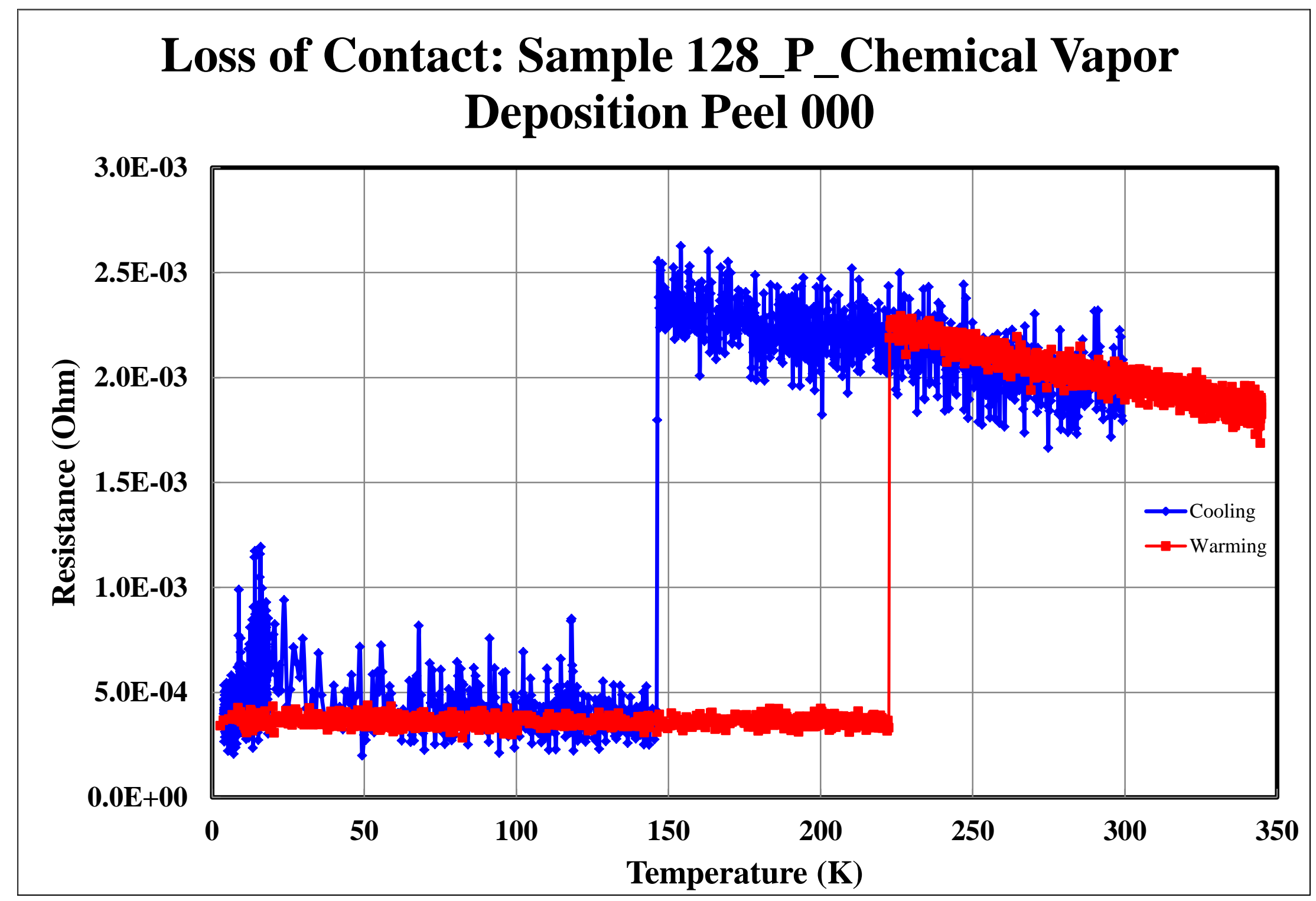


Appendix 5 As Low Peel 


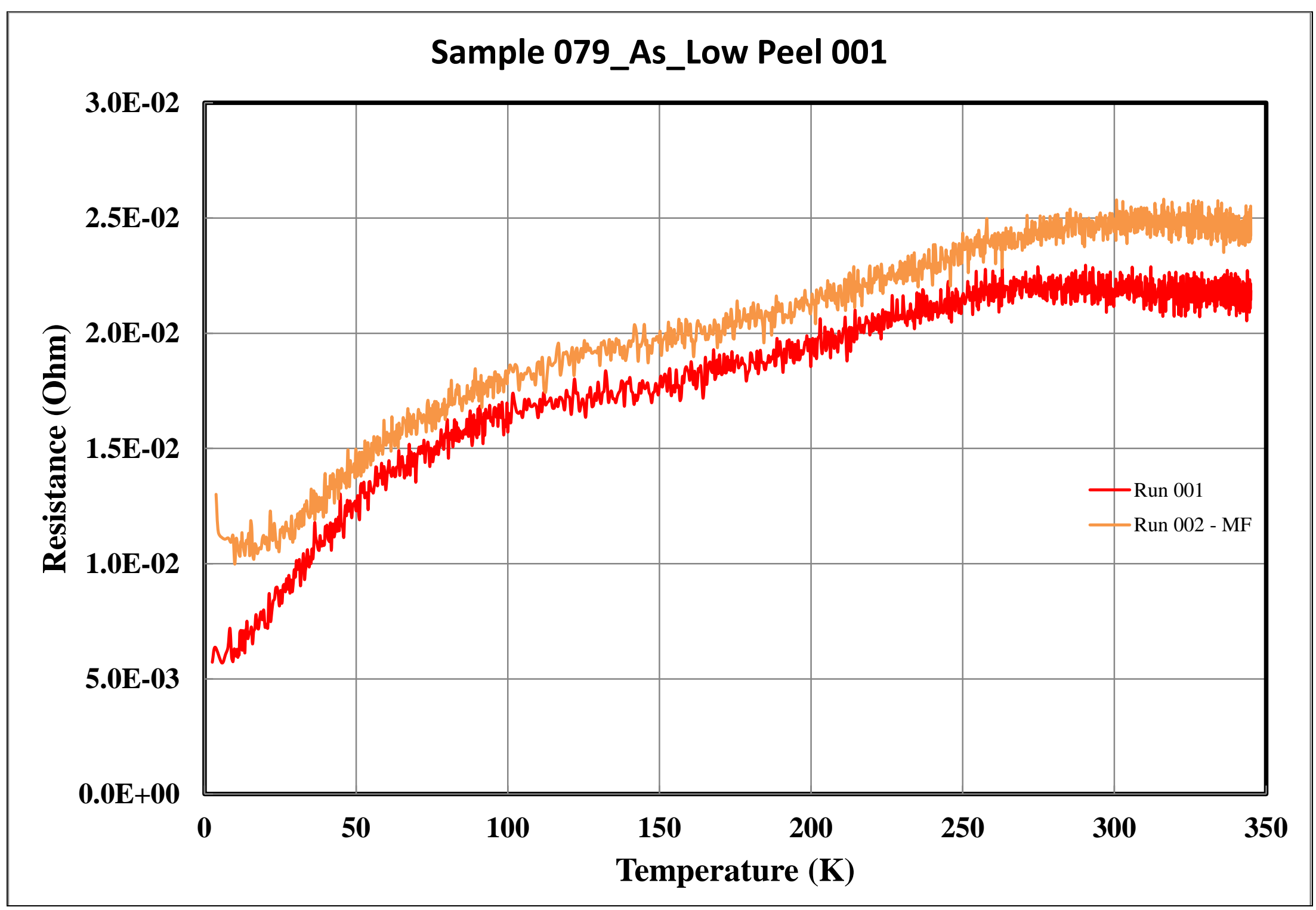


Appendix 6 As Hi Peel 


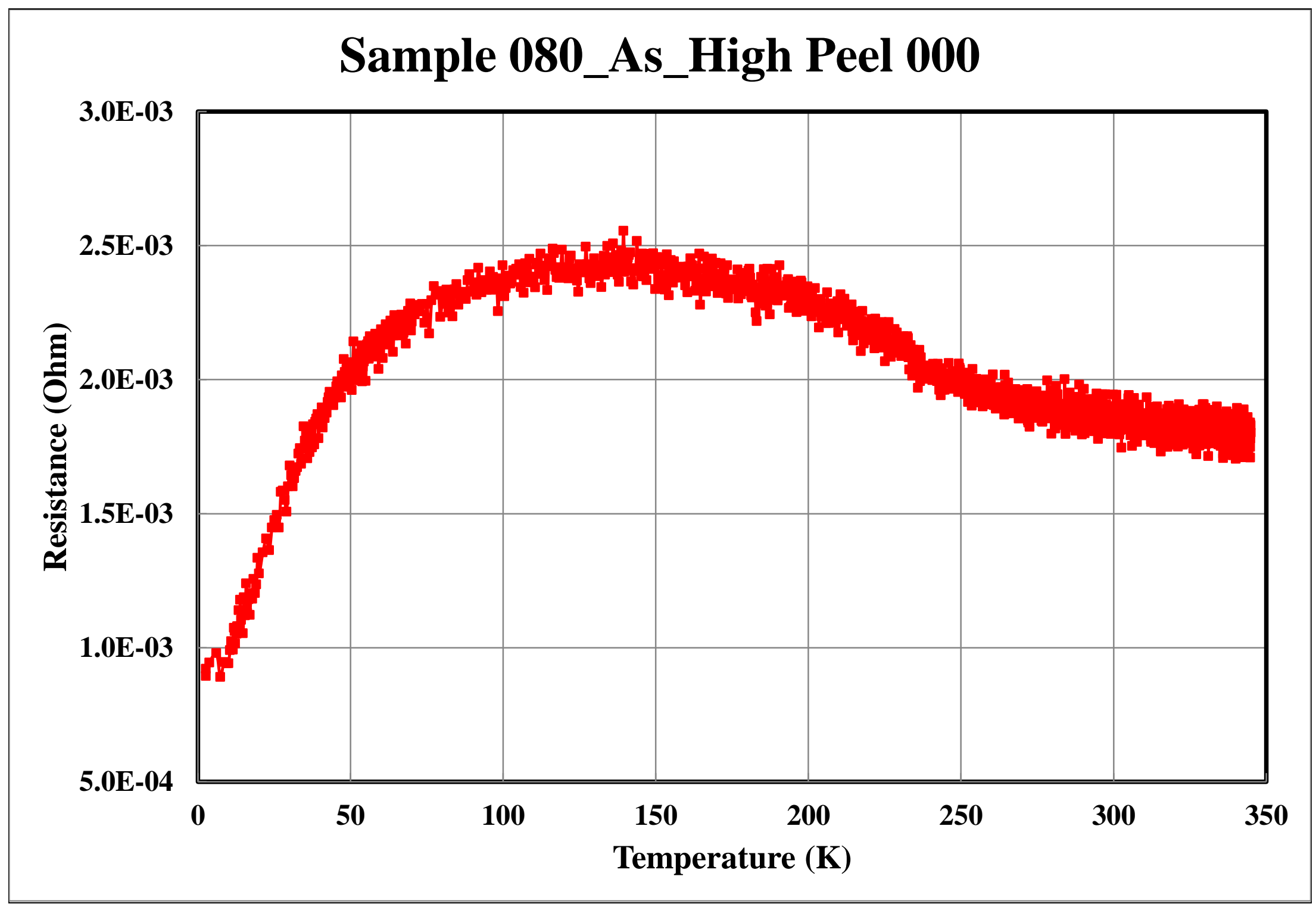


Appendix $7 \quad$ Al Low Bulk Run 002 and Run 003 - MF 


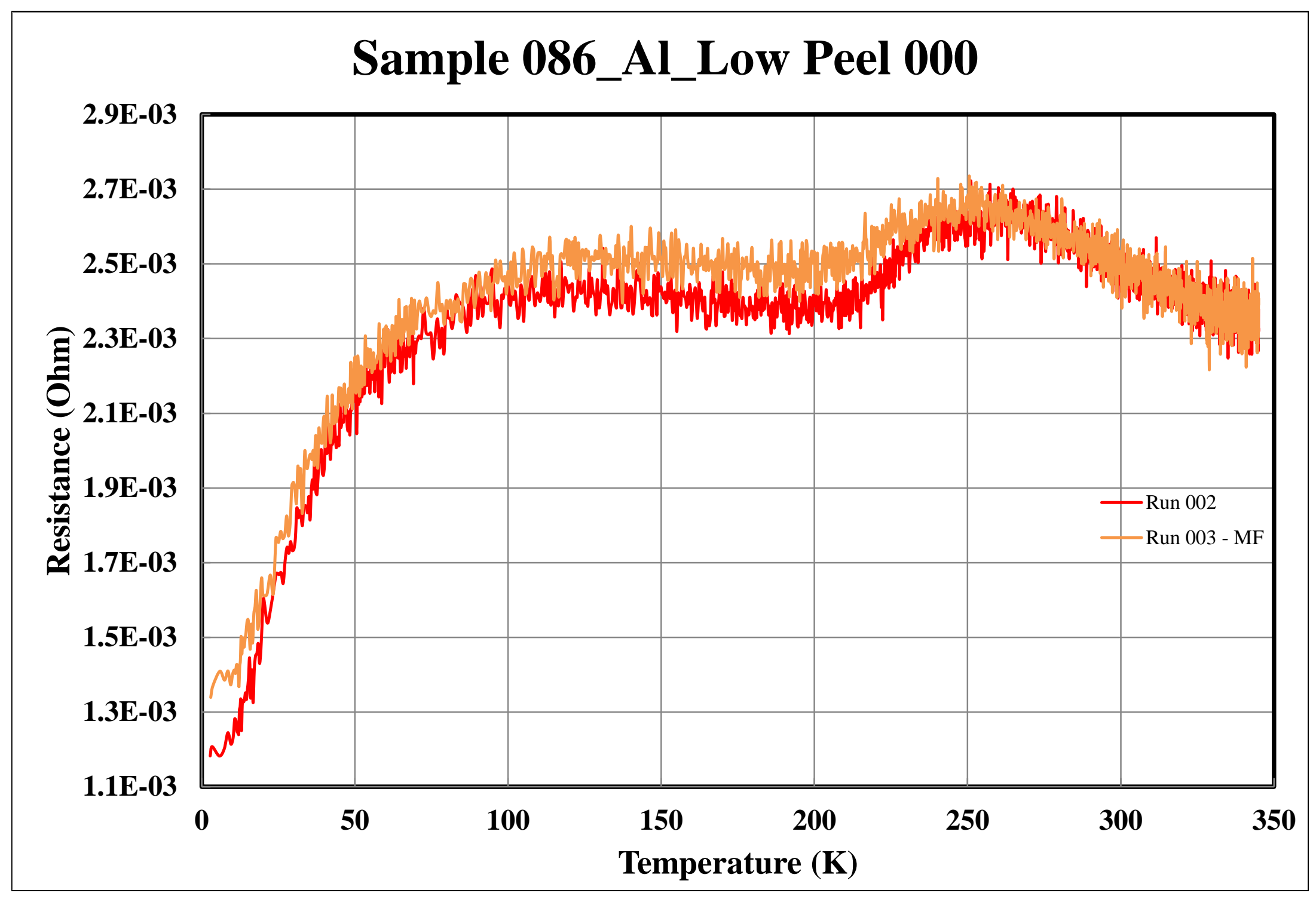


Appendix 8 Al Low Bulk Run 003-MF and Run 004 


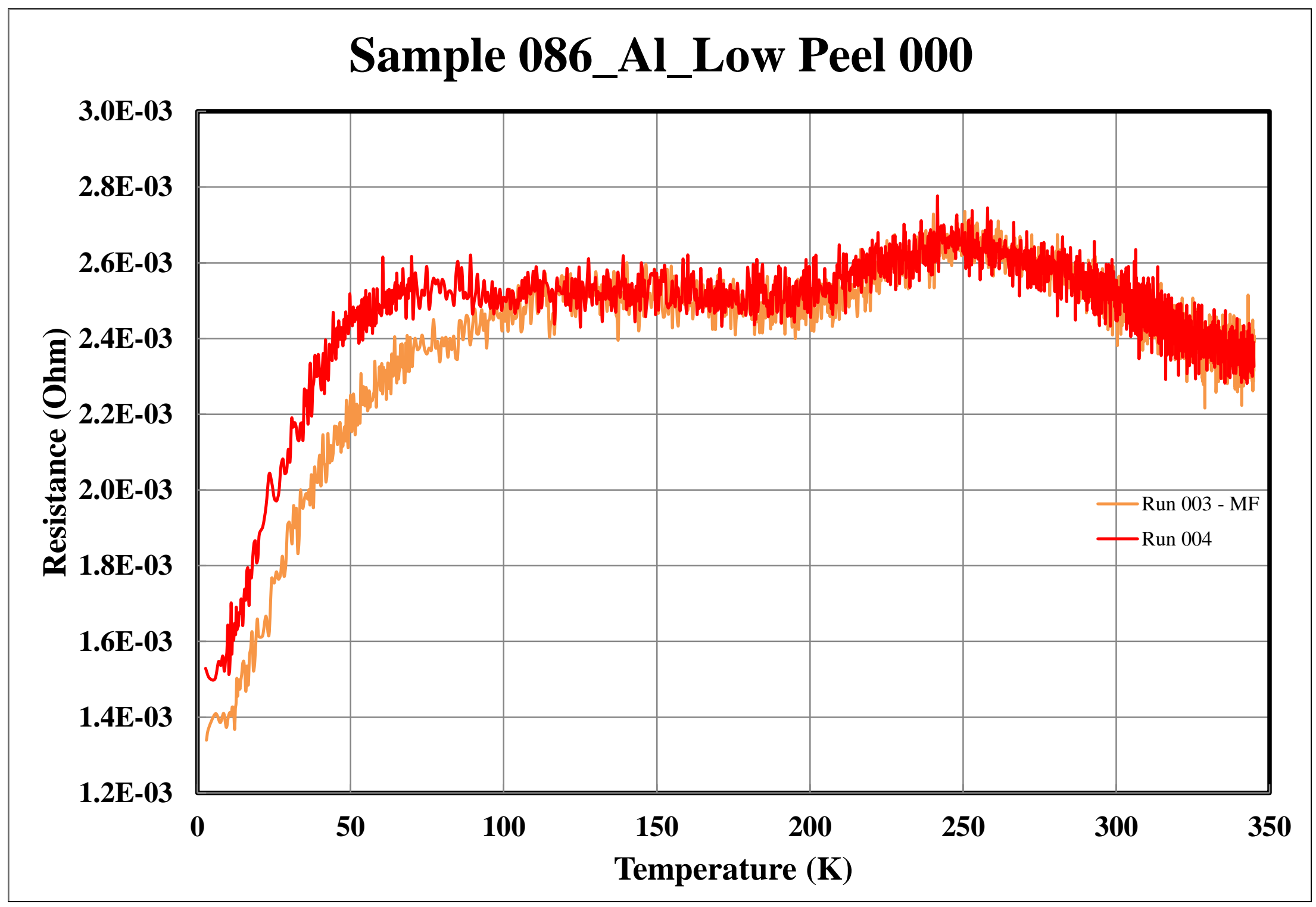




\section{Appendix $9 \quad$ Al High}




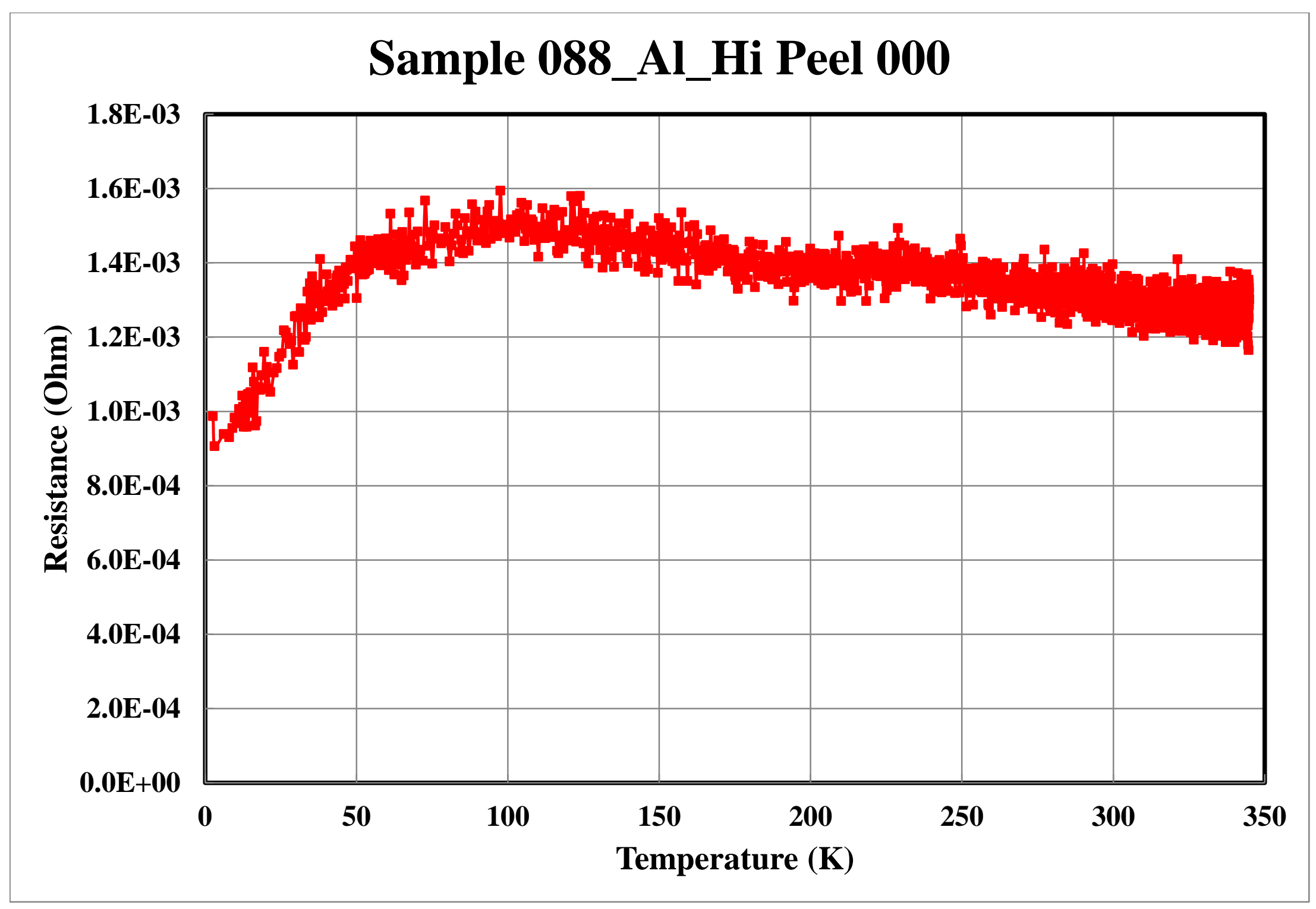


Appendix $10 \quad$ N Low, Peel 000, Run 002 and Run 003 - MF 


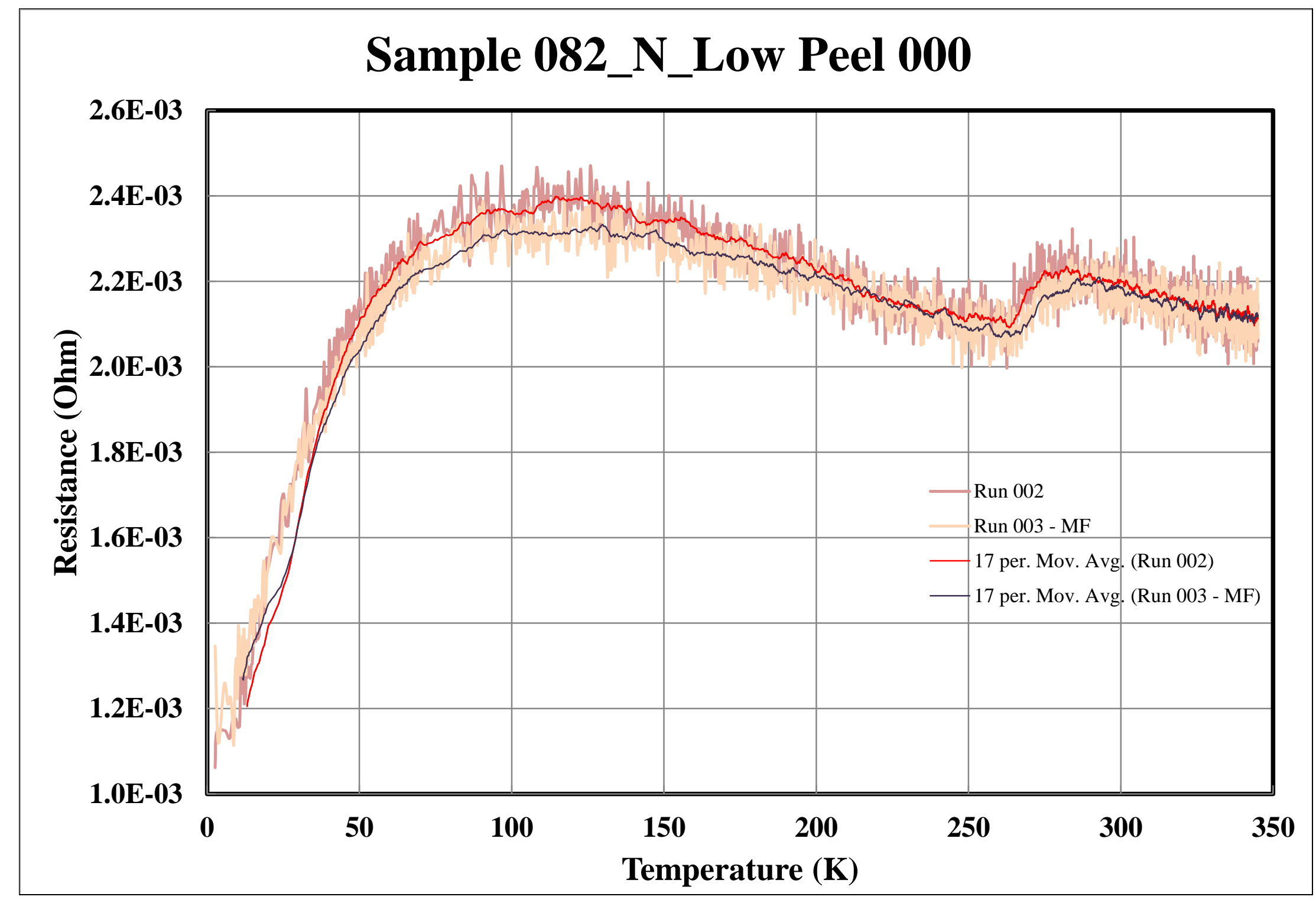


Appendix $11 \quad$ N Low, Peel 000, Run 003 - MF and Run 004 


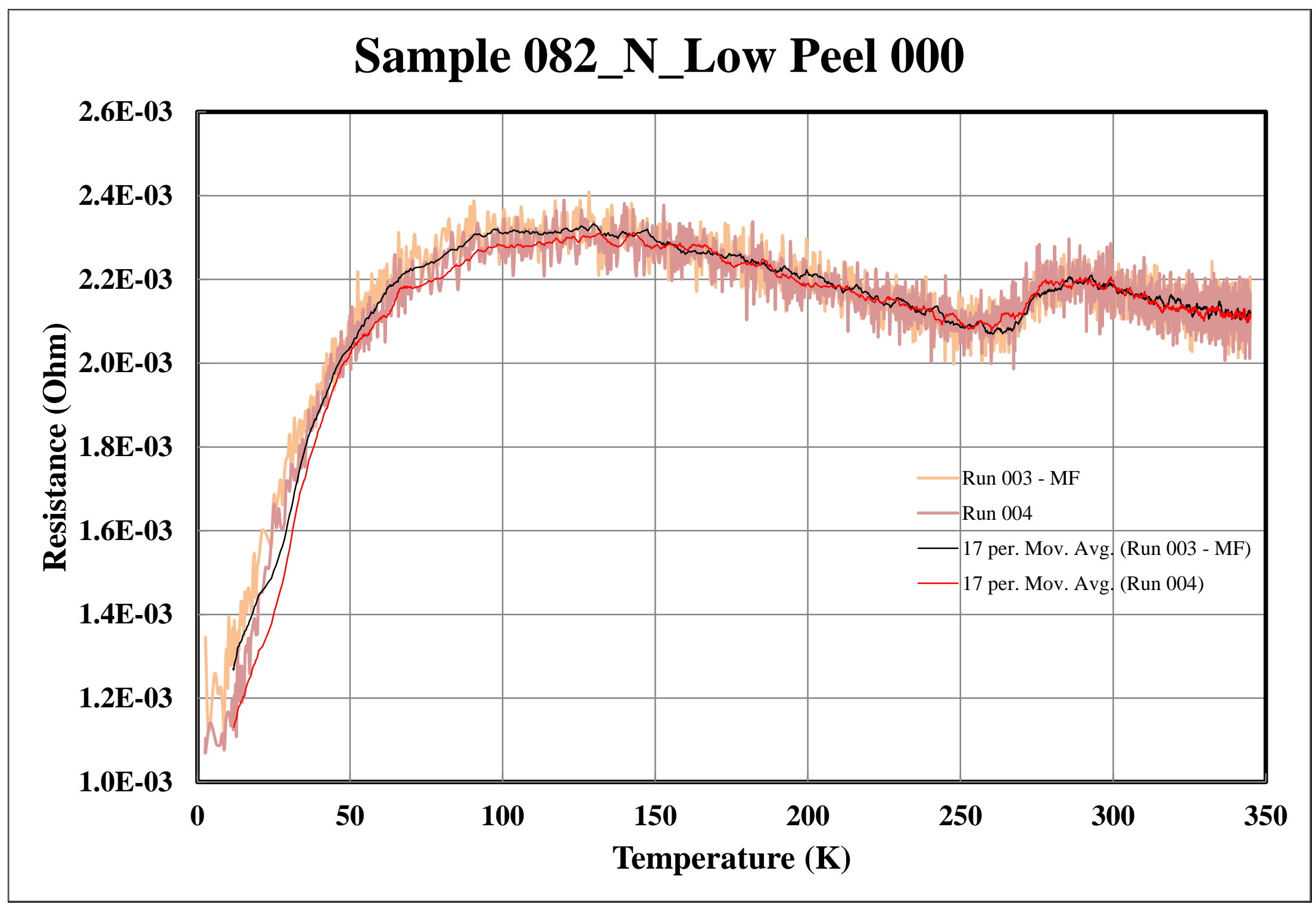


Appendix 12 N Low, Peel 003, Run 002 and Run 003 - MF 


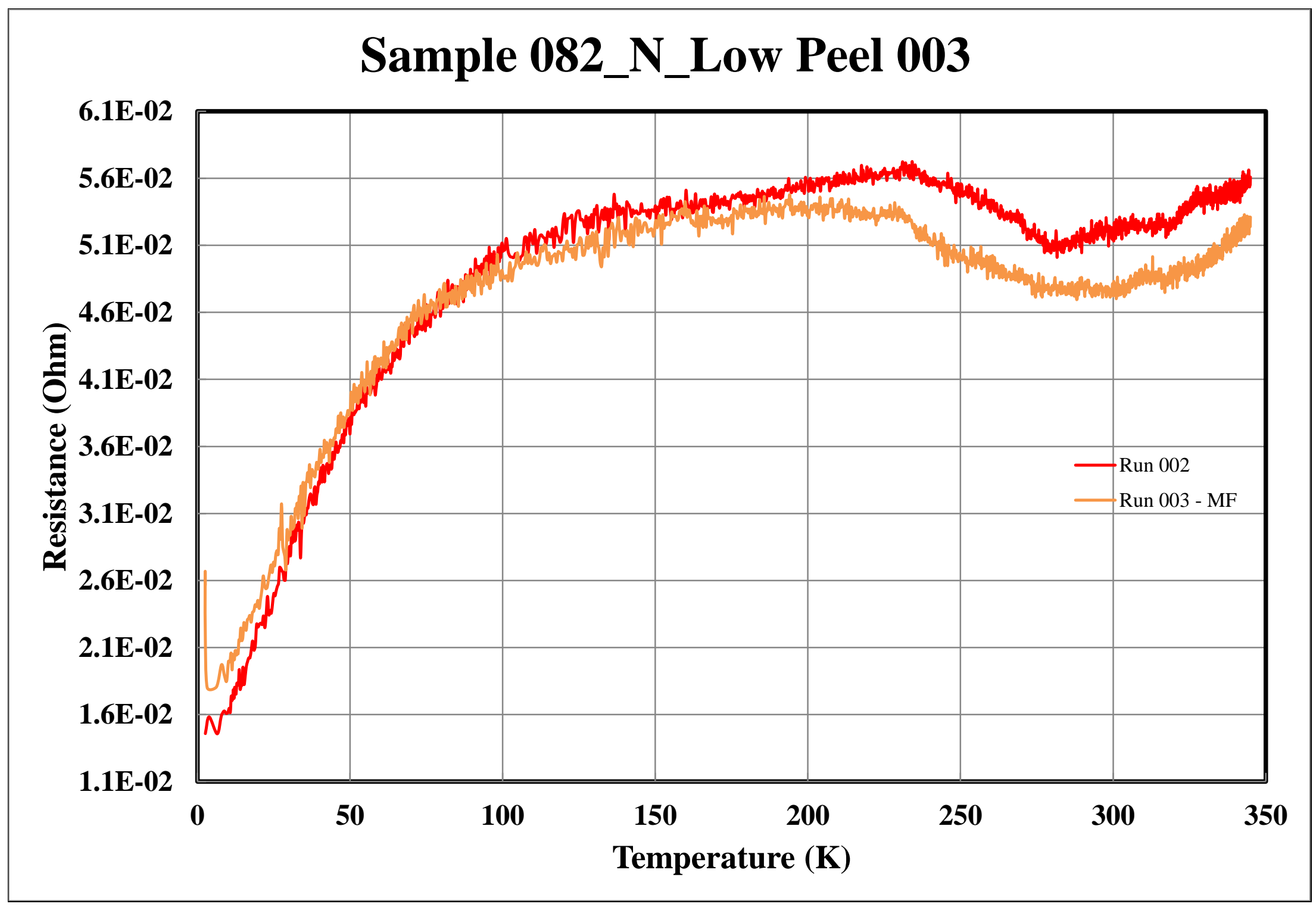


Appendix 13 N Low, Peel 003, Run 003 - MF and Run 004 


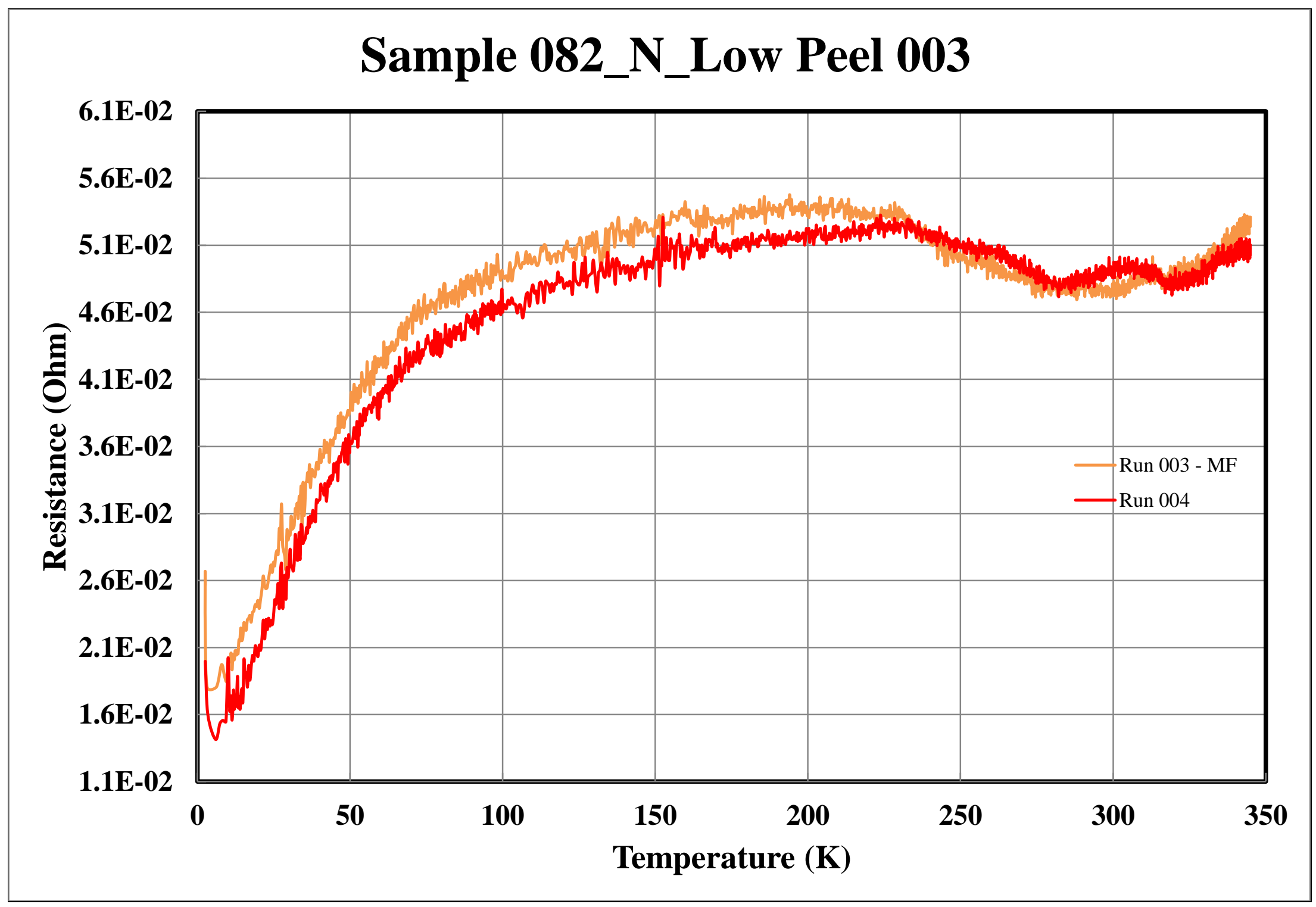


Appendix $14 \quad$ N High 


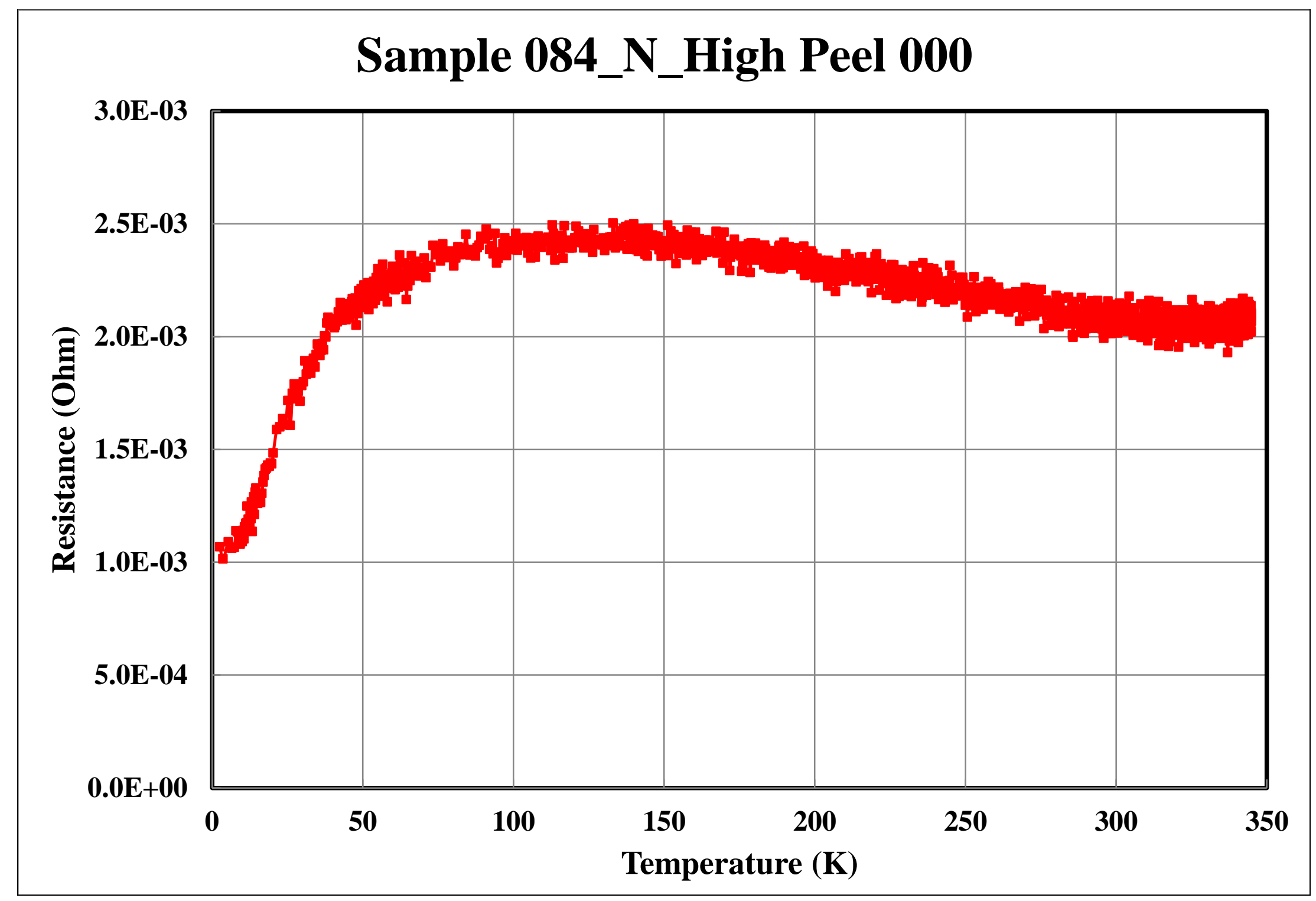


Appendix 15 Be Low 


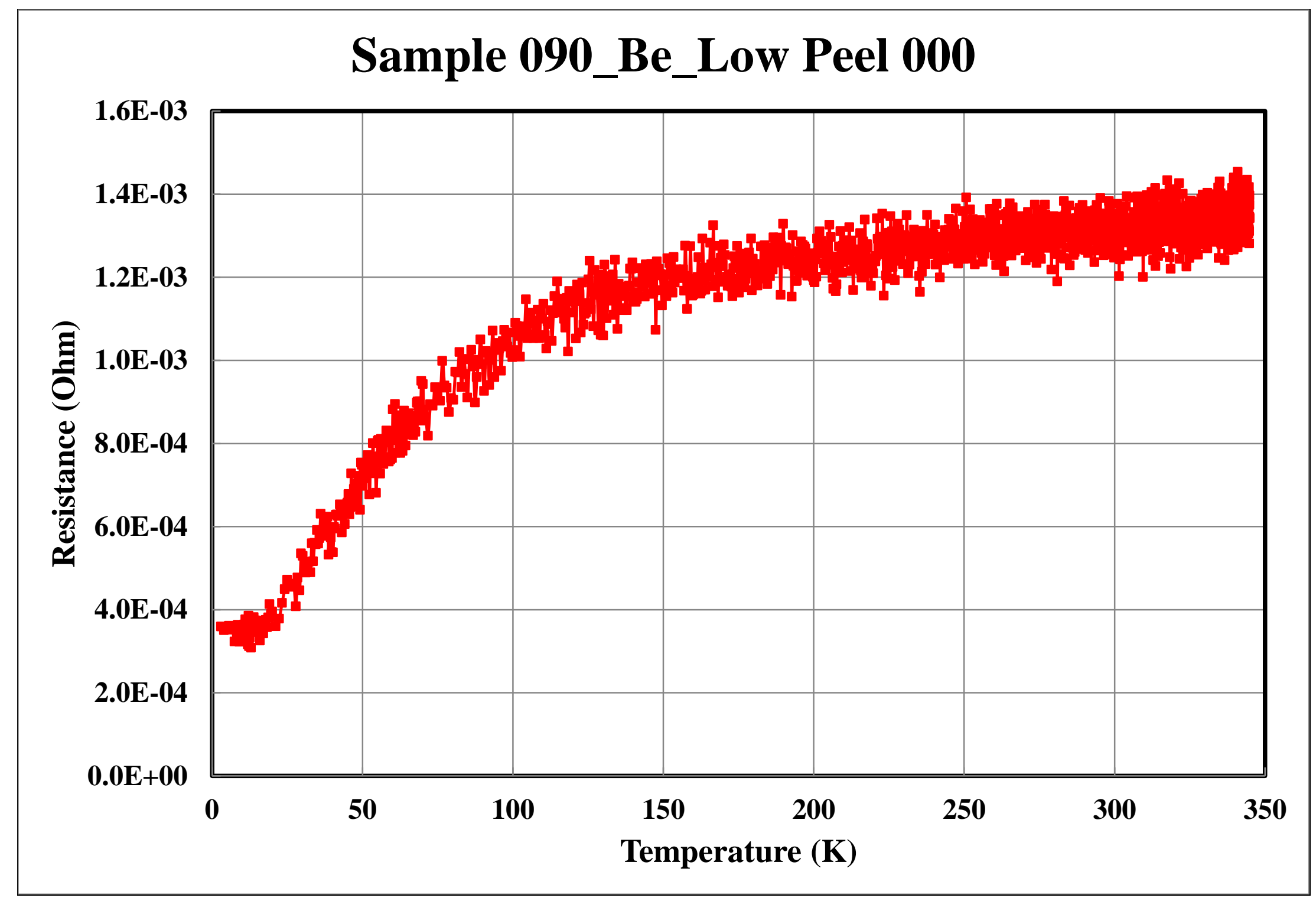


Appendix 16 Be High 


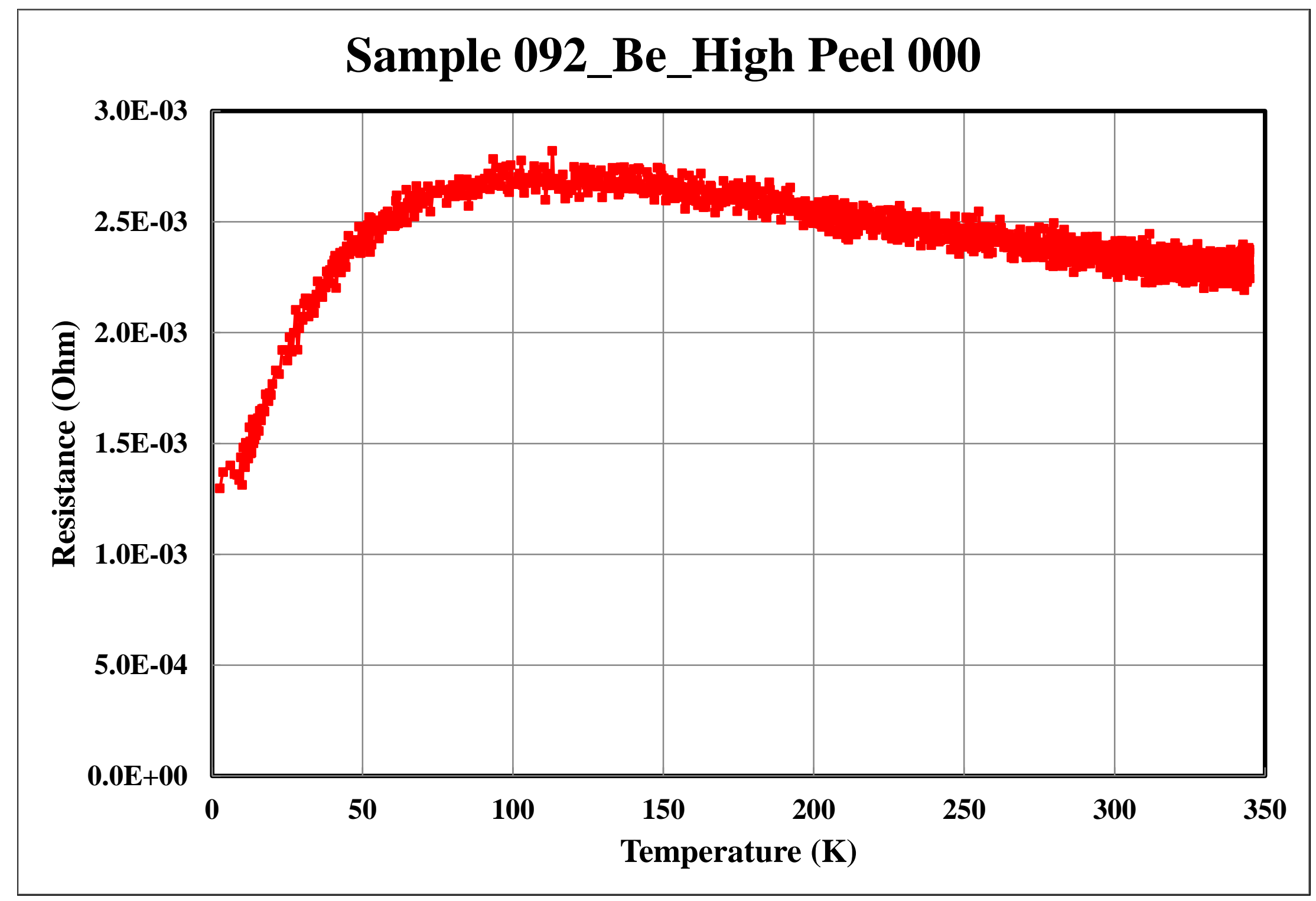


Appendix $17 \quad$ F Low 


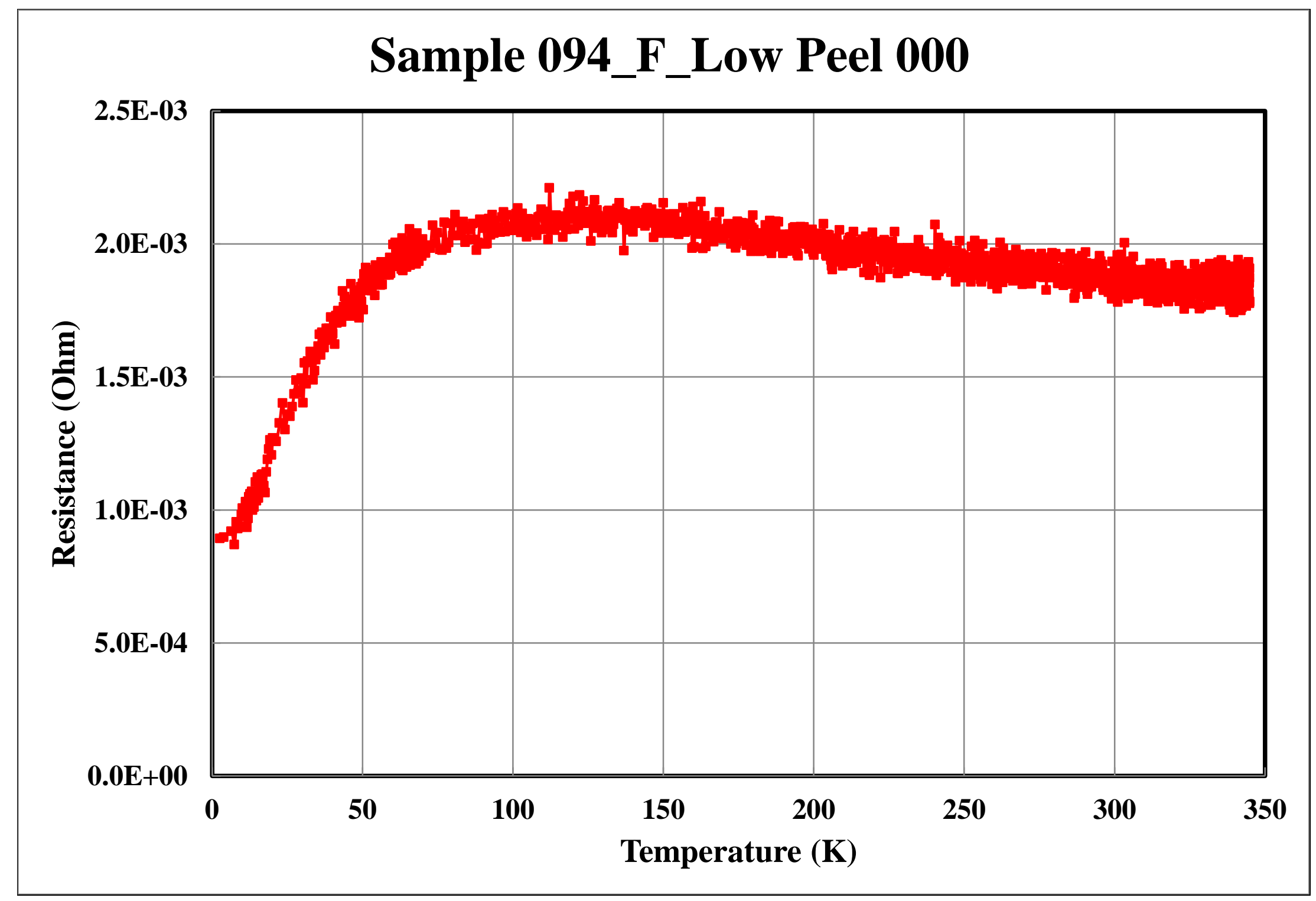


Appendix $18 \quad$ F High 


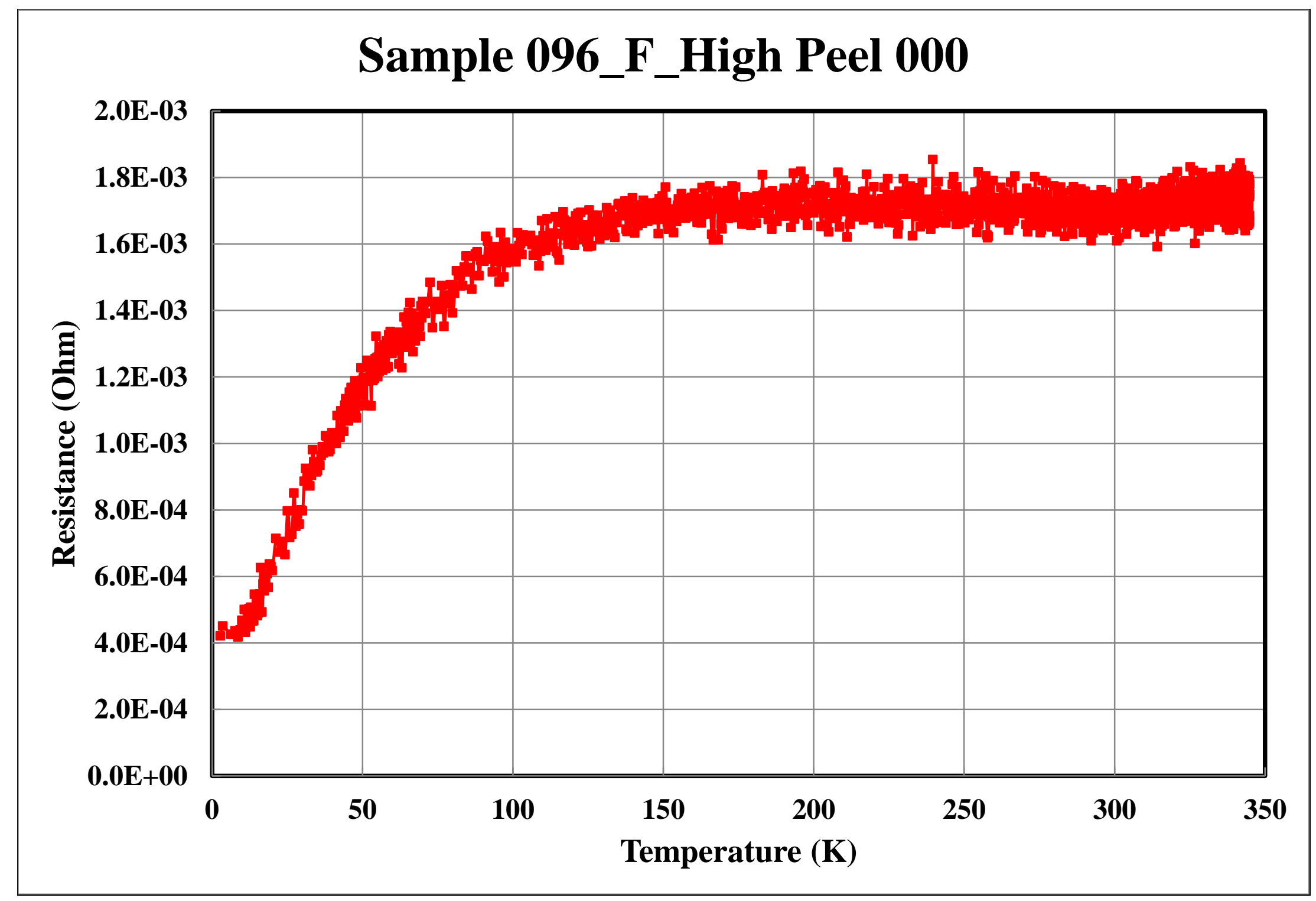


Appendix $19 \quad$ Li Low 


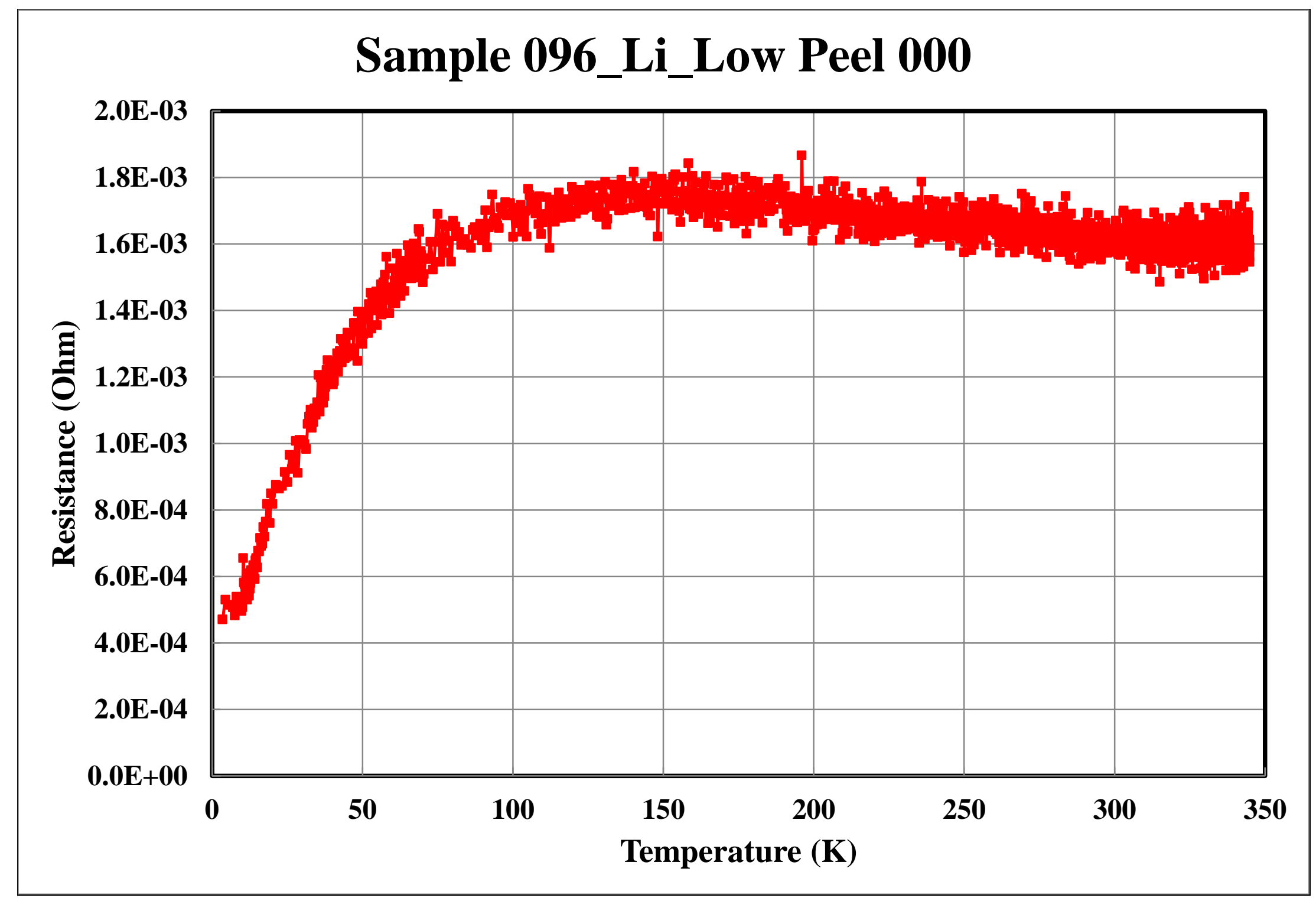


Appendix $20 \quad$ Li High 


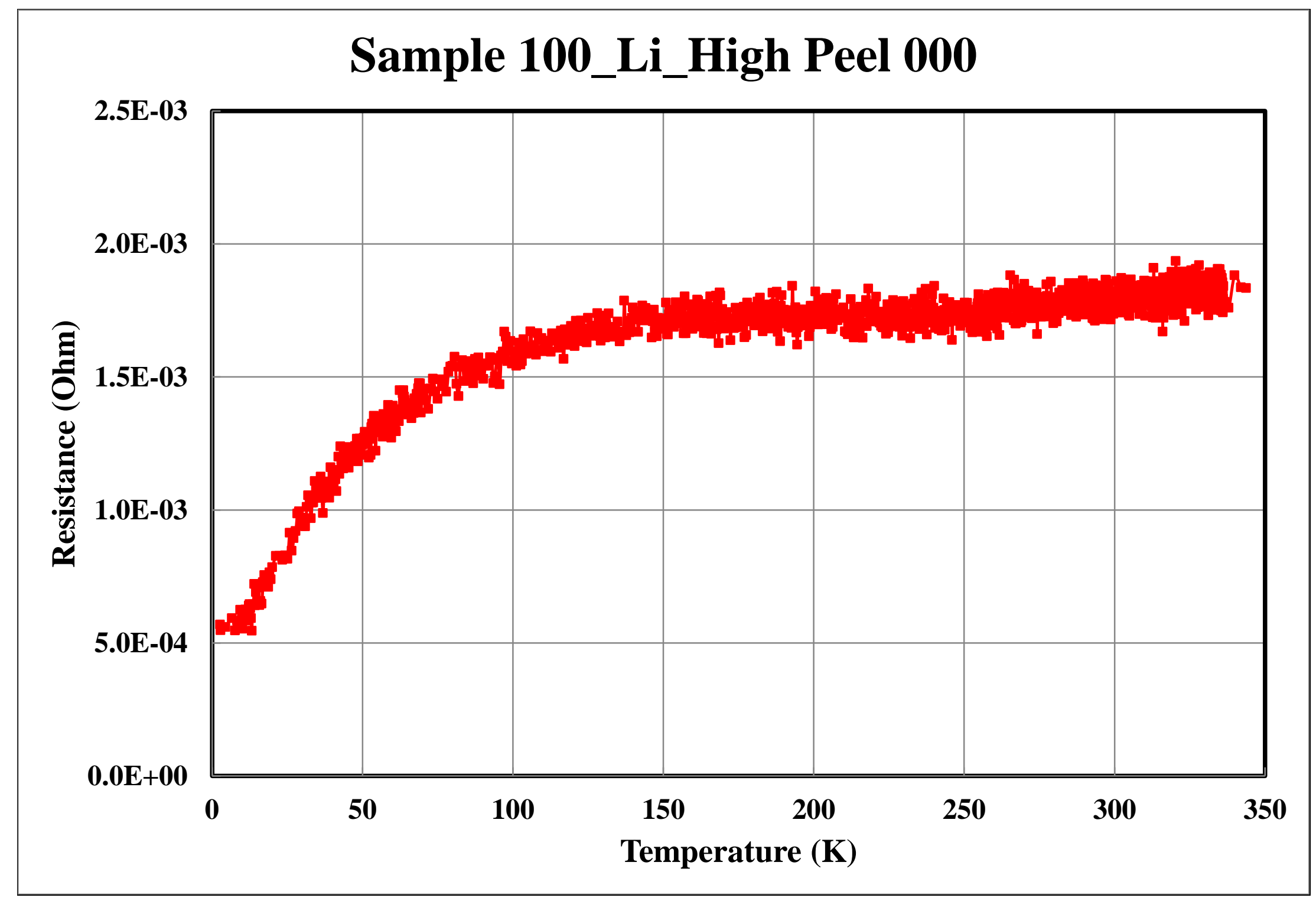


Appendix $21 \quad$ Na Low 


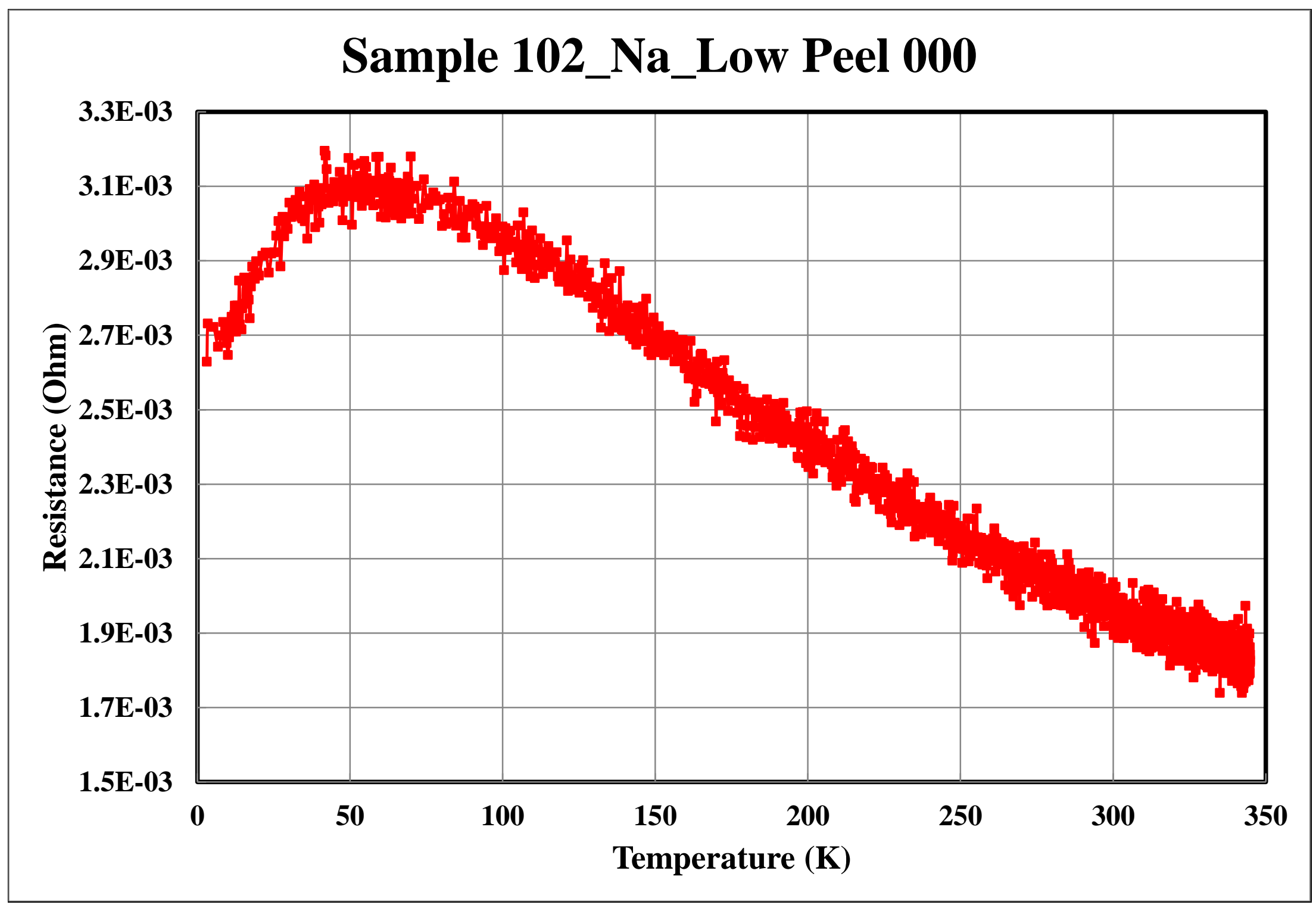


Appendix 22 Na High 


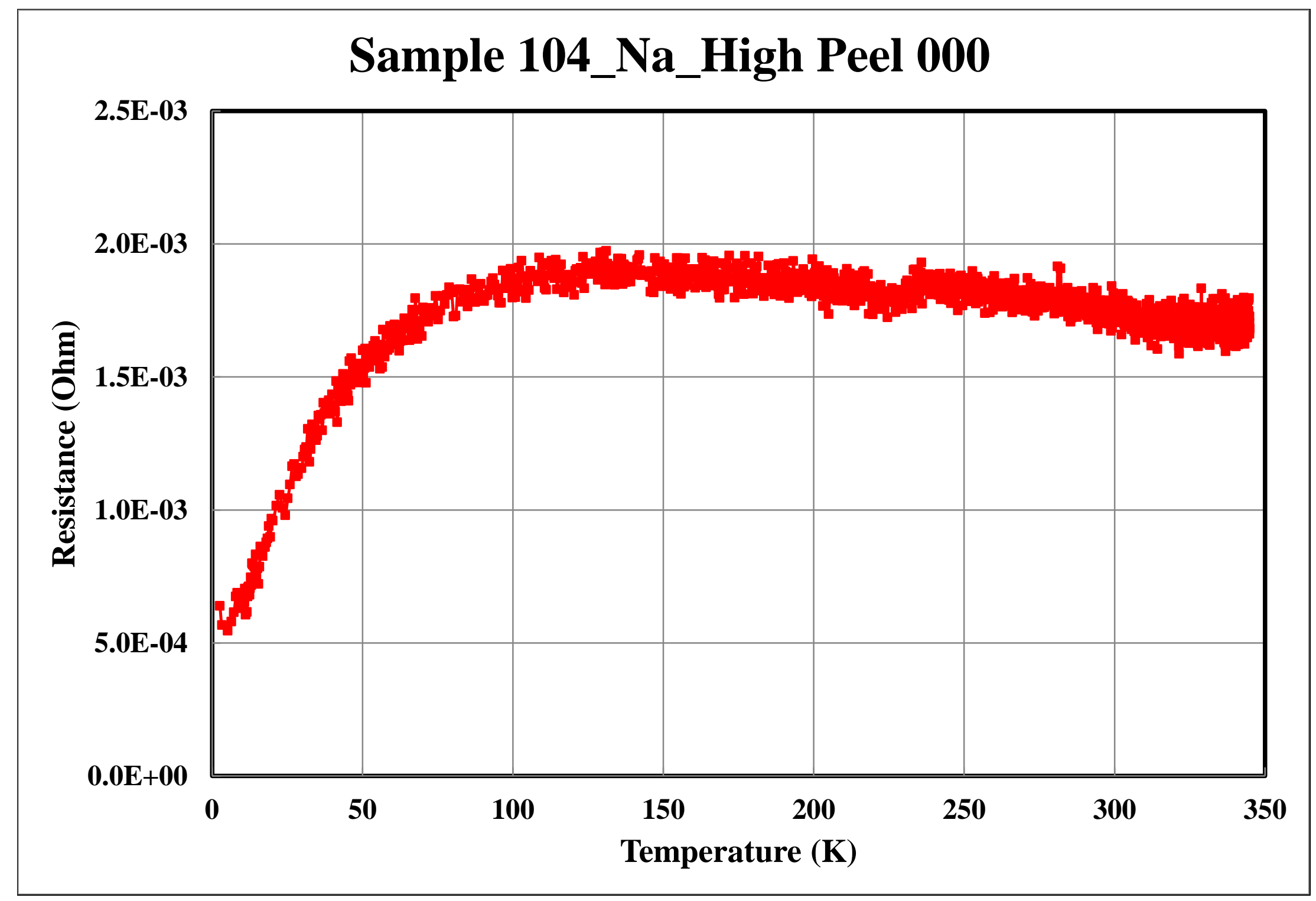


Appendix 23 S Low 


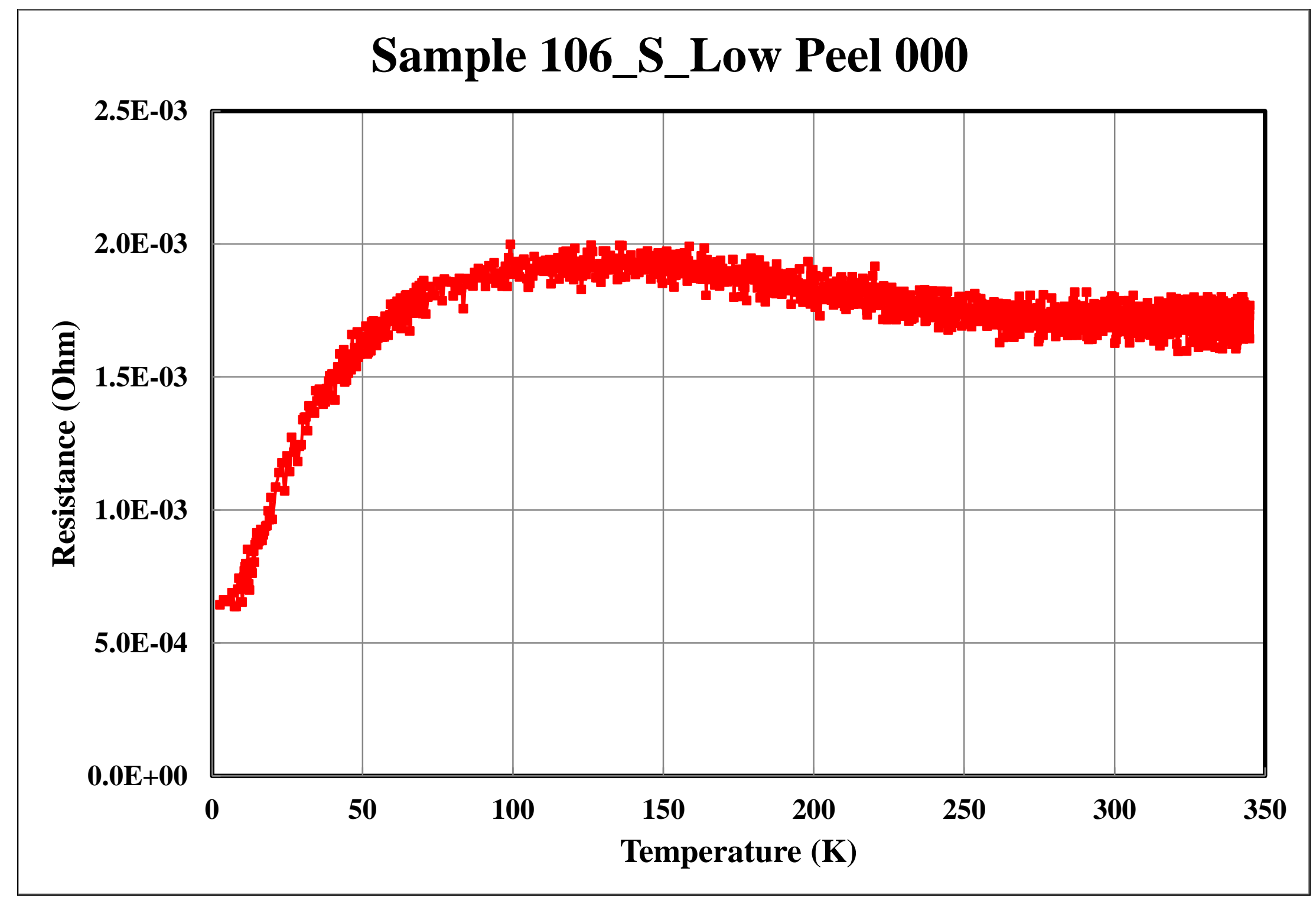


Appendix 24 S High 


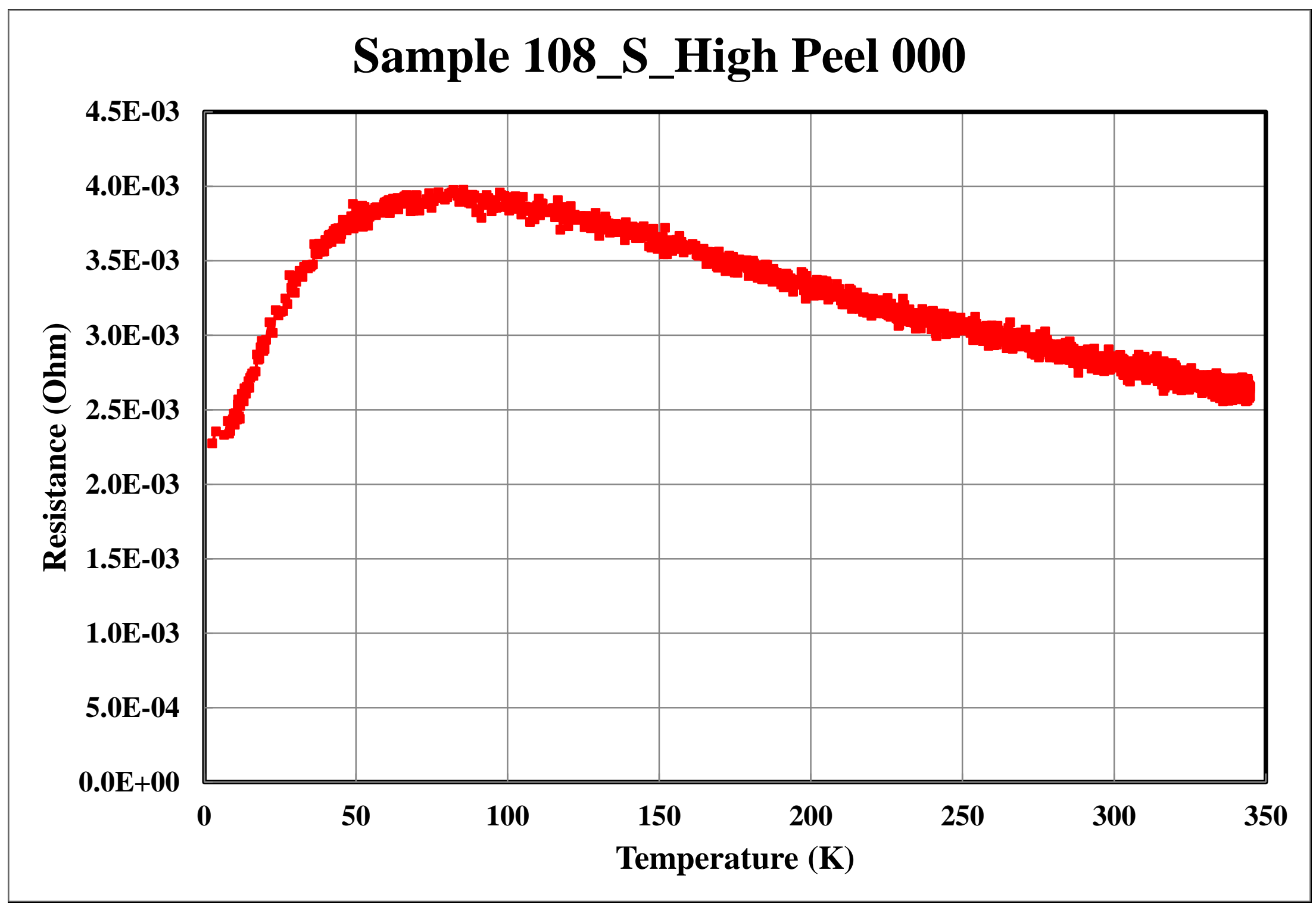


Appendix 25 Flow Chart for Cryo4-Delta Measure ver 23 Auto compressor and two separate files generated- VI name was disconnected on cooling in previous version.vi 
User inputs: When to turn on heaters, MF, Sample \#, Layer \#, Current, Operator, Comments.

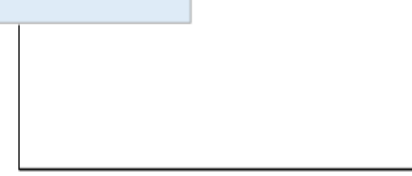

Turn Compressor On

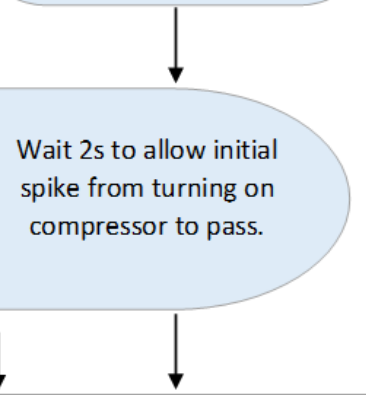

SubVI for R vs. T. Takes user inputs, takes measurements until cooling is complete, while relaying measurements to front panel.

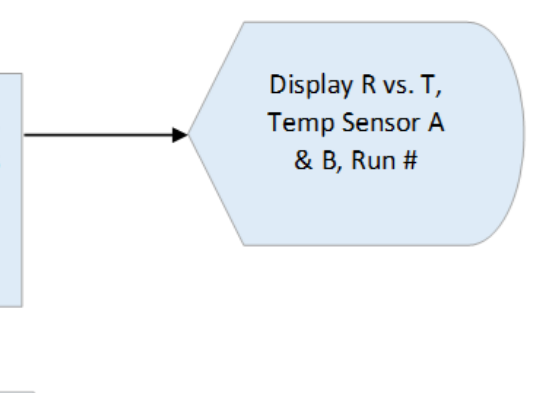

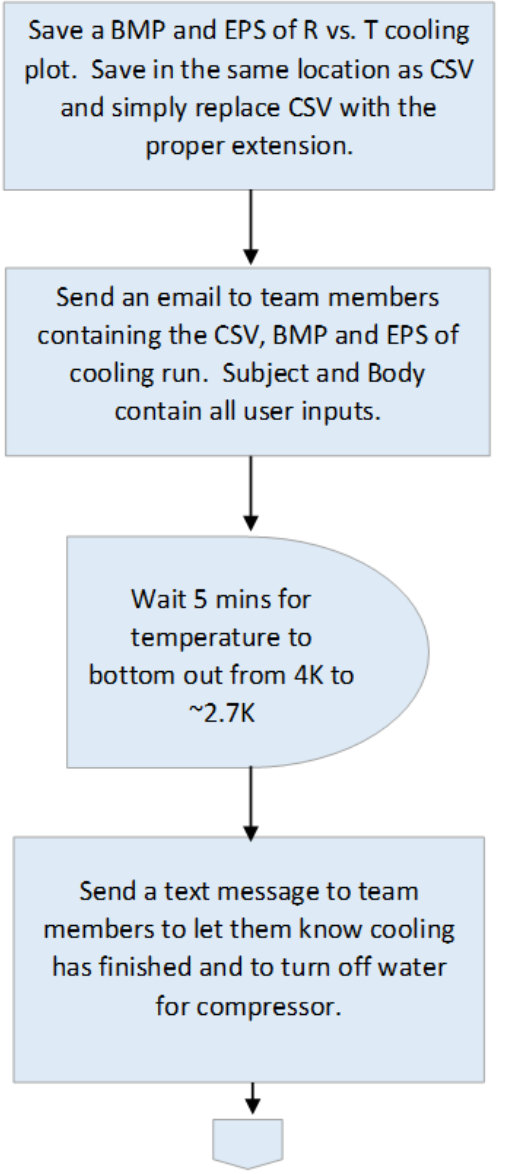




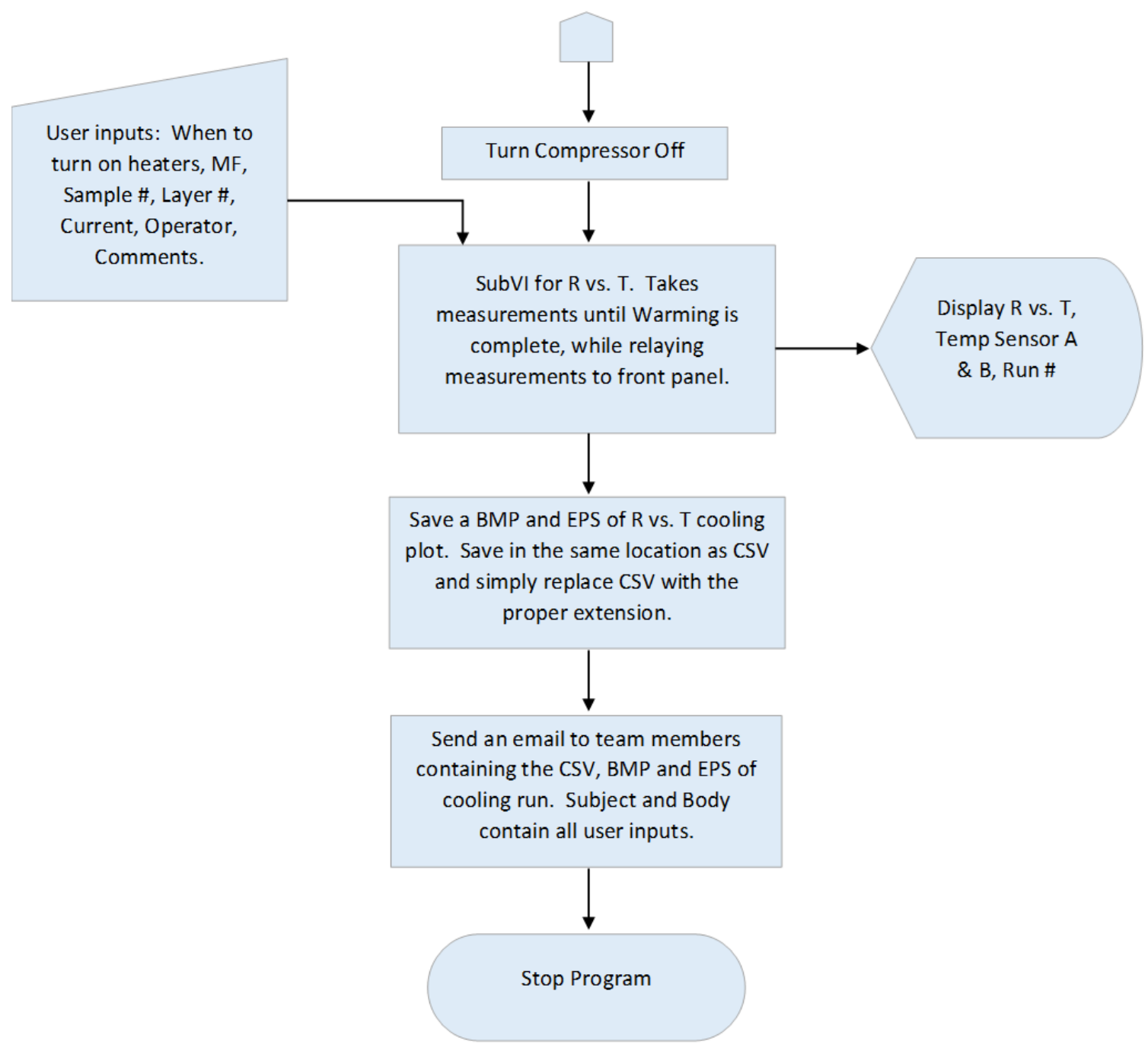


Appendix 26 Flow Chart For - Header Gen (SubVI) version 2 - run logger

attempt to add comments.vi 


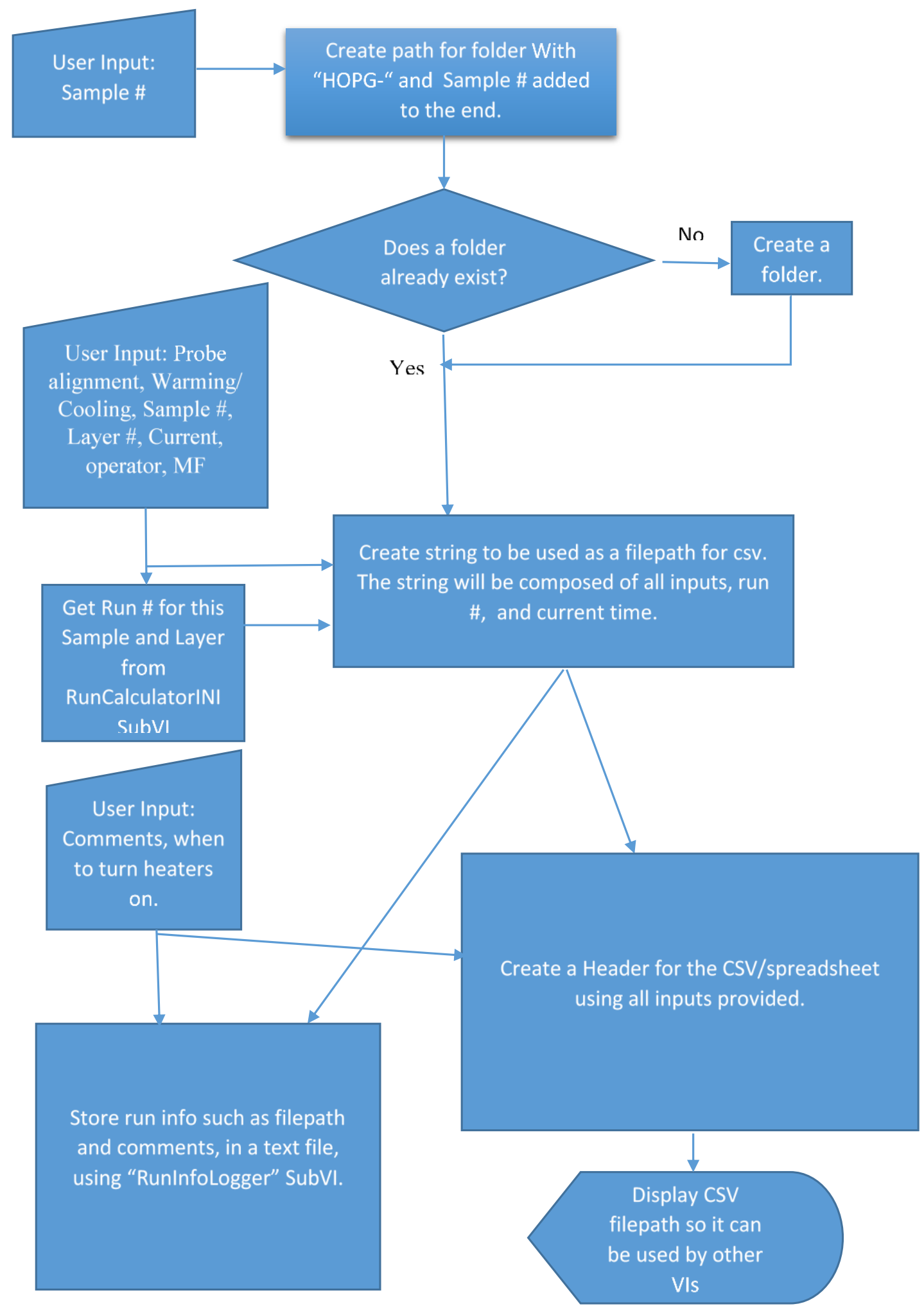


Appendix 27 Flow Chart For - R vs T SubVI LakeShore part - SubVI for R

vs T run v3 - Case structure removed.vi 


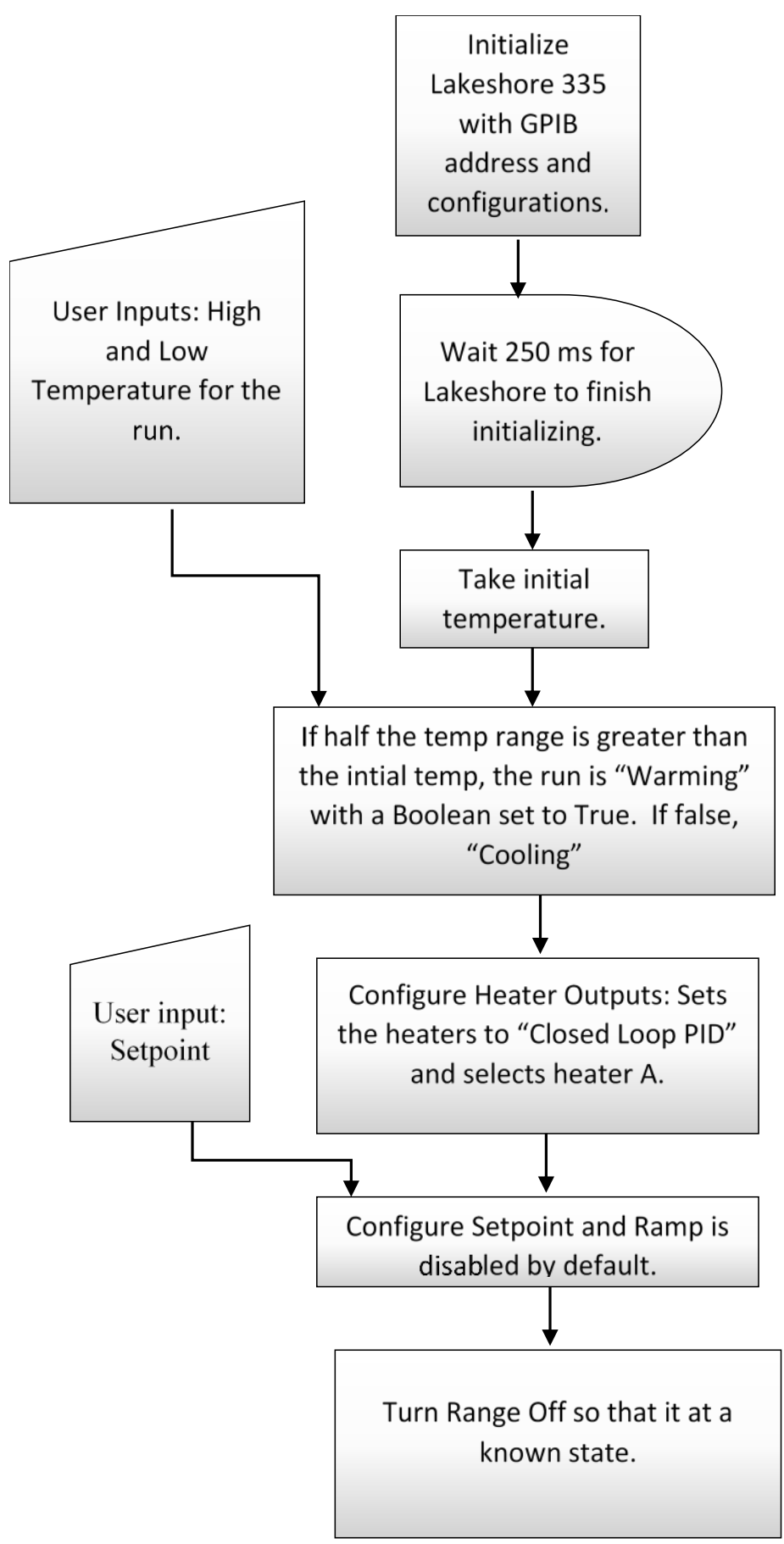


Appendix 28 Flow Chart For - RunCalculatorINI.vi 


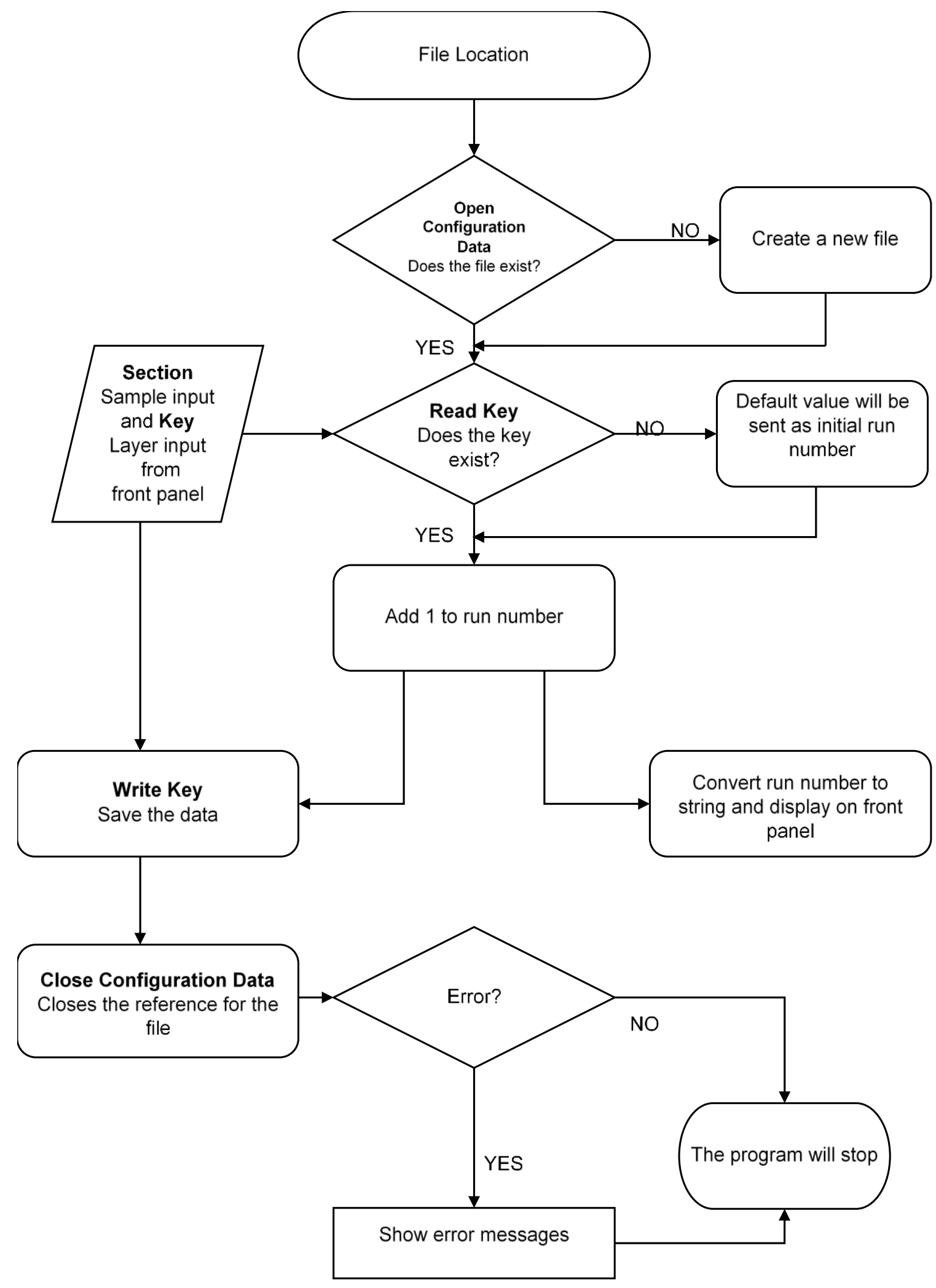


Appendix 29 Cryo3- Magnetometer Ver 2.vi 
Cryo3- Magnetometer Ver 2-1.vi

C:\Users\LabVIEW-user\AppData\Local\Temp\Cryo3- Magnetometer Ver 2-1.vi Last modified on 5/20/2016 at 5:55 PM

Printed on 5/20/2016 at 5:57 PM

\section{Cryo3- Magnetometer Ver 2-1.vi}
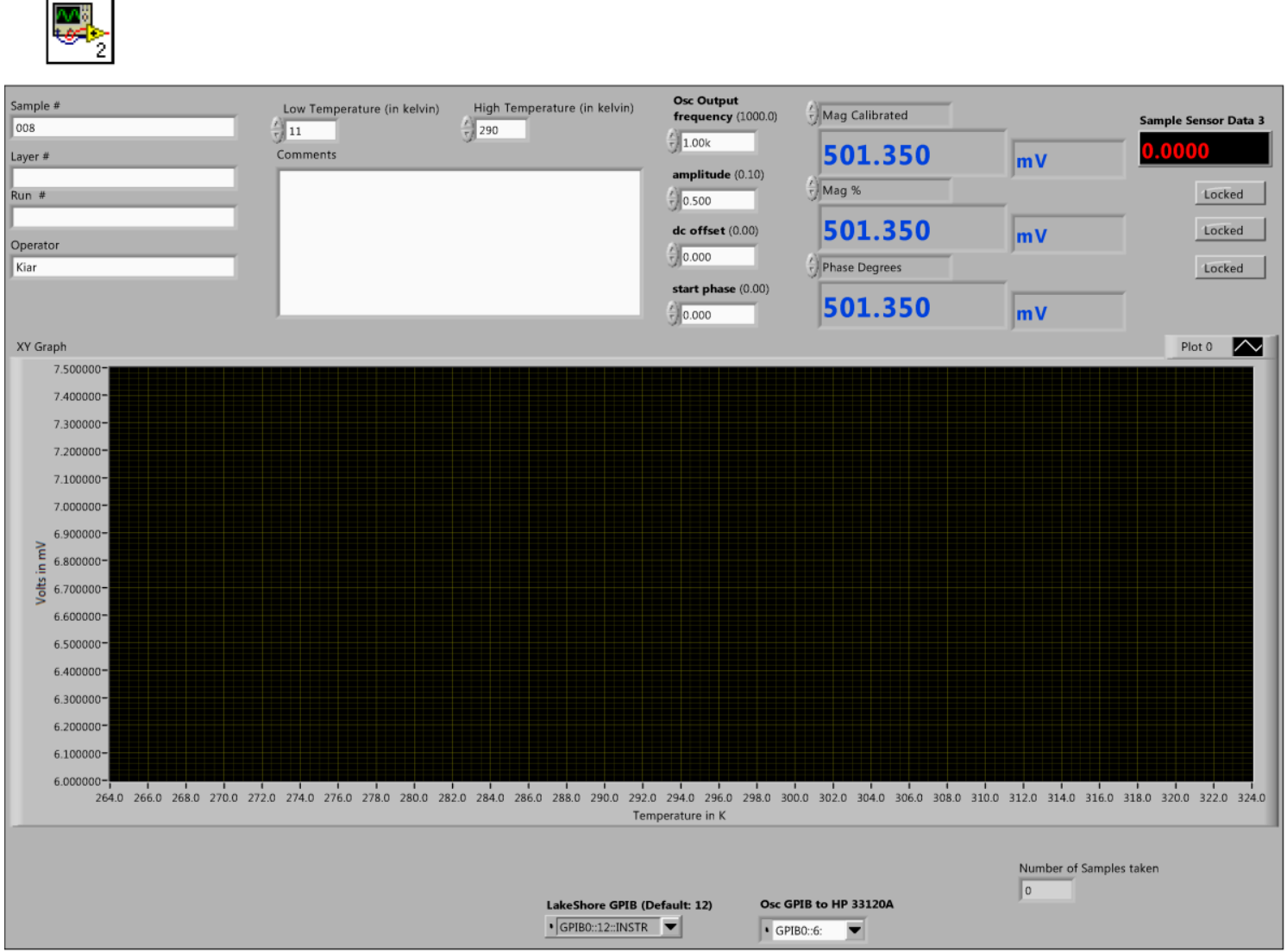
Cryo3- Magnetometer Ver 2-1.vi

Page 2

C:\Users\LabVIEW-user\AppData\Local\Temp\Cryo3- Magnetometer Ver 2-1.vi Last modified on 5/20/2016 at 5:55 PM

Printed on 5/20/2016 at 5:57 PM

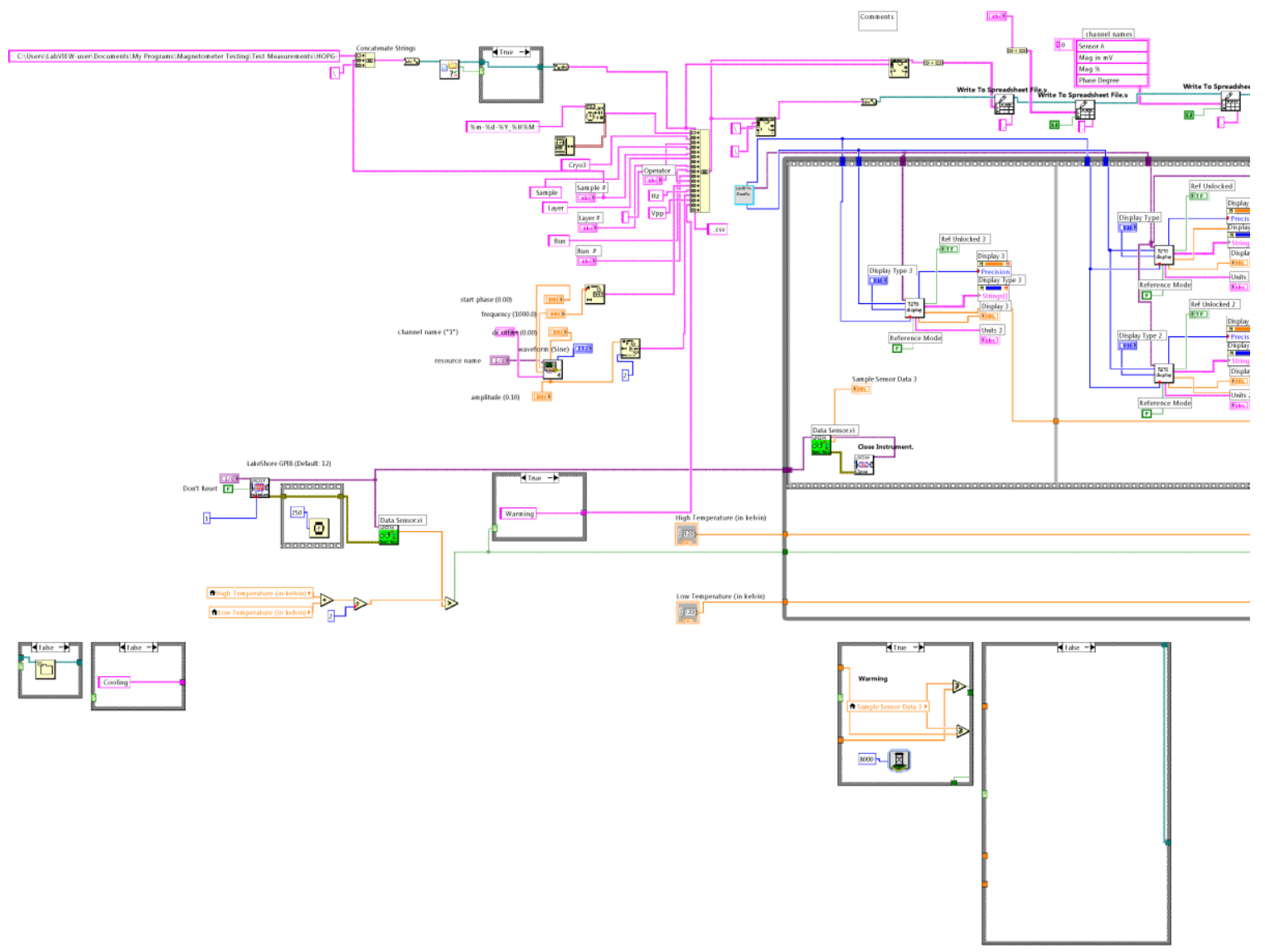

冈 Time Delay

Time Delay

Inserts a time delay into the calling VI.

-

This Express VI is configured as follows:

Delay Time: $30 \mathrm{~s}$

Build XY Graph

$2{ }_{10}$ Build XY Graph

formats the data displayed on an X-Y Graph. 
Cryo3-Magnetometer Ver 2-1.vi

C:\Users\LabVIEW-user\AppData\Local\Temp\Cryo3- Magnetometer Ver 2-1.vi Last modified on 5/20/2016 at 5:55 PM

Printed on 5/20/2016 at 5:57 PM

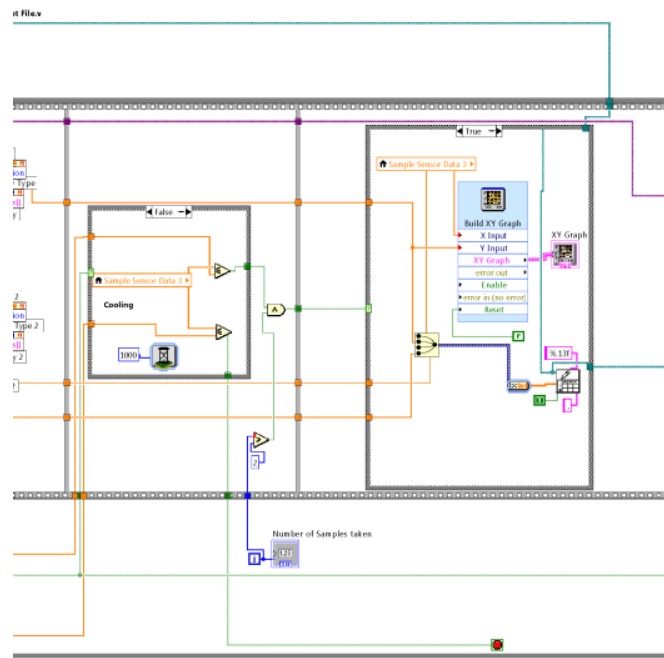

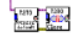

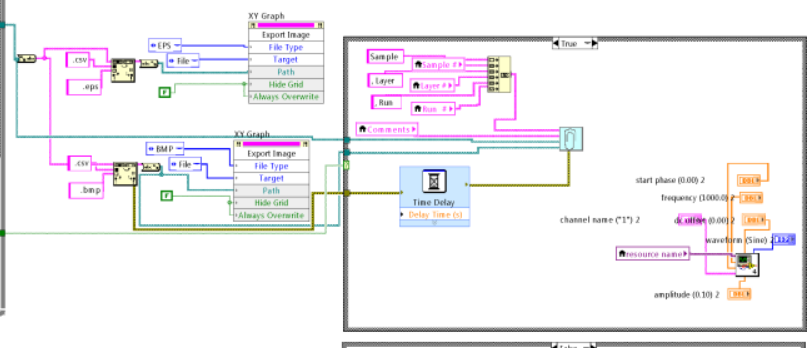


Cryo3- Magnetometer Ver 2-1.vi

C:\Users\LabVIEW-user\AppData\Local\Temp\Cryo3- Magnetometer Ver 2-1.vi

Last modified on 5/20/2016 at 5:55 PM

Printed on 5/20/2016 at 5:57 PM

Convert from Dynamic Data

Convert from Dynamic Data

Converts the dynamic data type to numeric, Boolean, waveform, and array data types for use with other VIs and functions.

Q Wait

Wait

Delays for a certain time interval before the output data dependence becomes valid.

冈 Wait2

Wait

Delays for a certain time interval before the output data dependence becomes valid. 
Appendix $30 \quad$ Cry03-SubVI: SR7270 8-bit R232 comms.vi 
SR7270 8-bit RS232 comms.vi

C:IUsers\LabVIEW-user\Desktop\KELVIN \& TUA\Kelvin\SR7270LV8.Ilb\SR7270 8-bit RS232 comms.vi Last modified on 5/11/2009 at 7:01 PM

Printed on 5/20/2016 at 8:01 PM

Connector Pane

\section{SR7270 8-bit RS232 comms.vi}

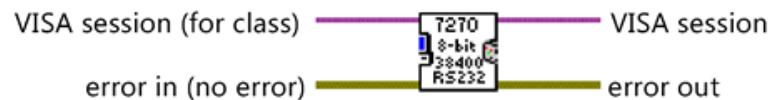

This VI forces the RS232 settings in the instrument and current VISA session, if this is using RS232, to 38400 baud ar 8 data bits, allowing subsequent binary dump commands, that respond with 8-bit data, to work correctly.

12 May 2009: First release

Front Panel

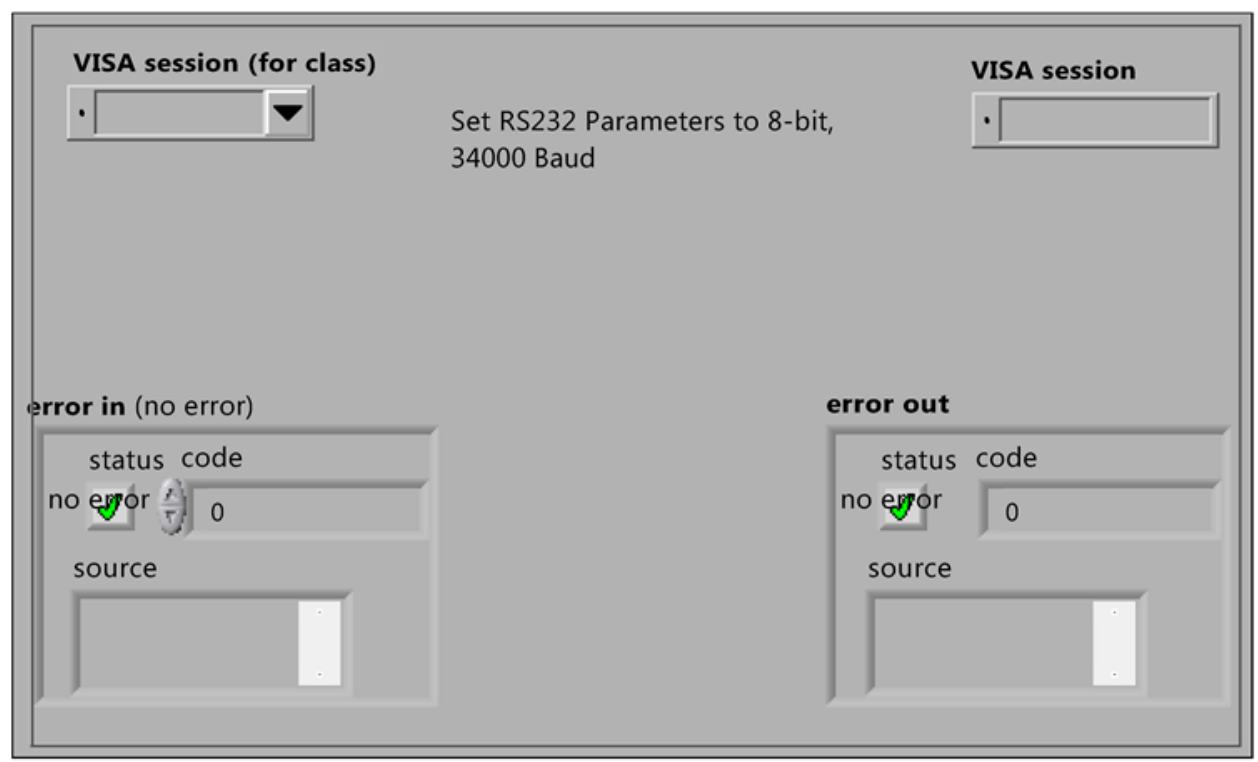


SR7270 8-bit RS232 comms.vi

C:\Users\LabVIEW-user\Desktop\KELVIN \& TUA\Kelvin\SR7270LV8.Ilb\SR7270 8-bit RS232 comms.vi Last modified on 5/11/2009 at 7:01 PM

Printed on 5/20/2016 at 8:01 PM

Block Diagram
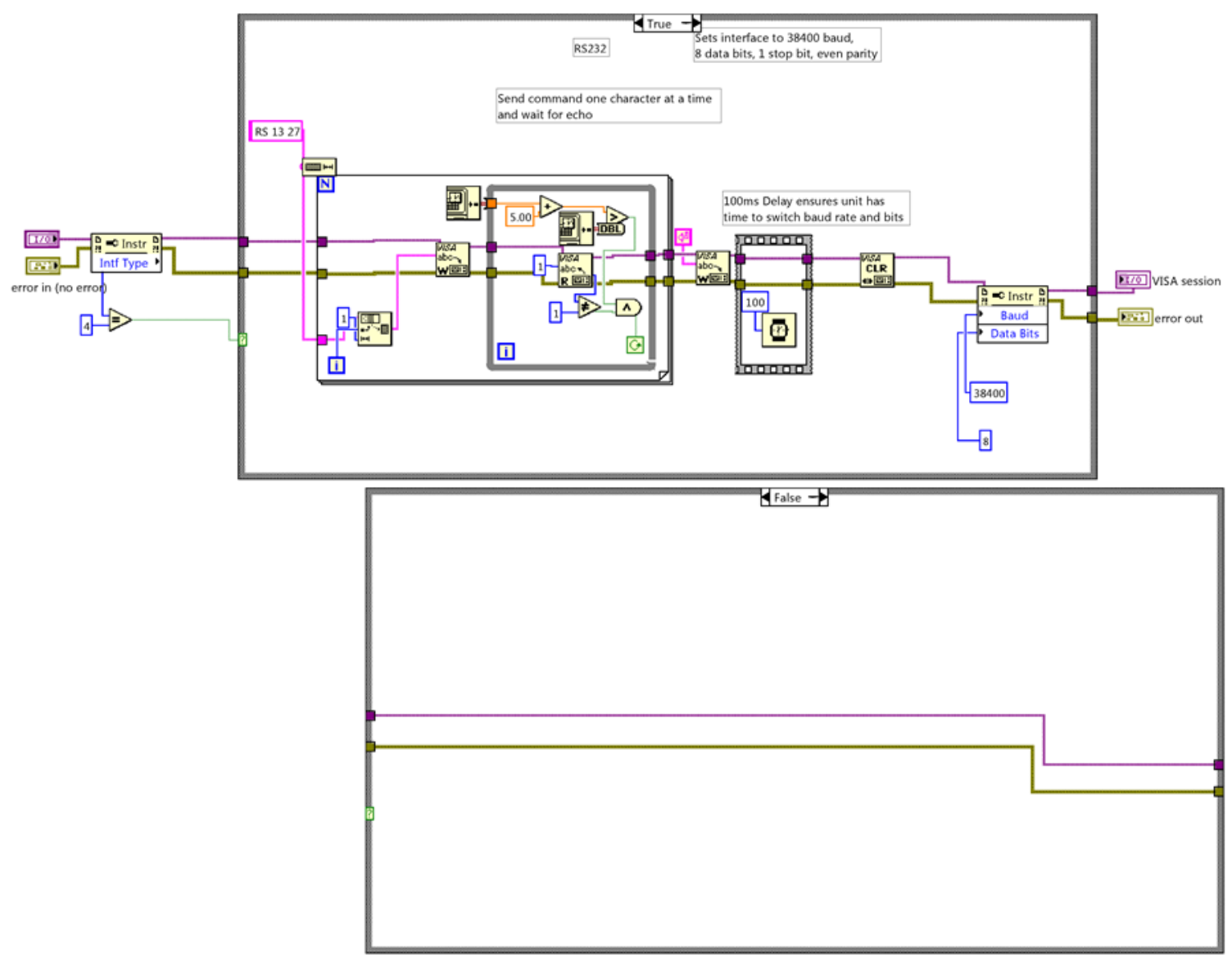
Appendix 31 Cryo3-SubVI: SR7270 Configure Reference Channel-Single

\author{
Reference.vi
}


SR7270 Configure Reference Channel - Single Reference.vi

Page $1 \quad \begin{gathered}7270 \\ \text { ref } \\ \text { chan }\end{gathered}$

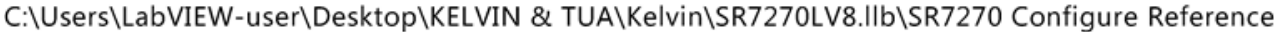

Channel - Single Reference.vi

Last modified on 5/11/2009 at 6:58 PM

Printed on 5/20/2016 at 8:02 PM

Connector Pane

SR7270 Configure Reference Channel - Single Reference.vi

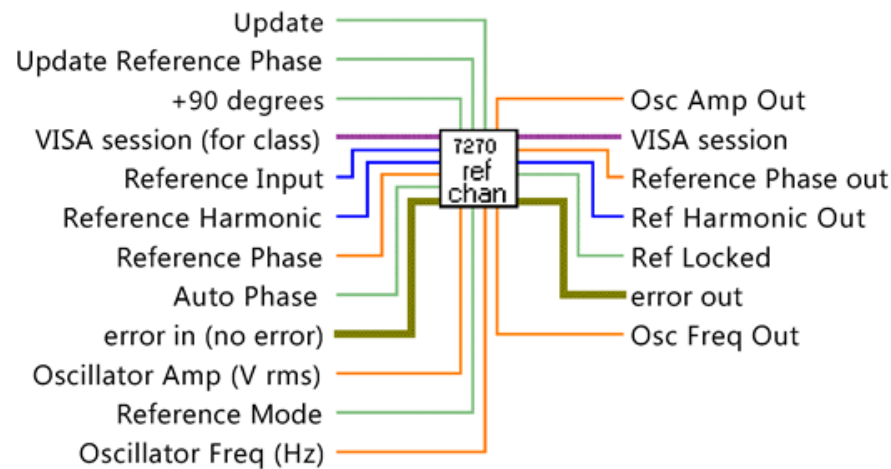

This VI controls the reference channel signal source, oscillator amplitude and frequency, reference phase and harmonic for either a single reference channel or for channel 1 when using the dual reference or dual harmonic modes

12 March 2009: First Release 
SR7270 Configure Reference Channel - Single Reference.vi

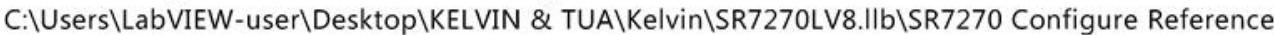

Channel - Single Reference.vi

Last modified on 5/11/2009 at 6:58 PM

Printed on 5/20/2016 at 8:02 PM

\section{Front Panel}

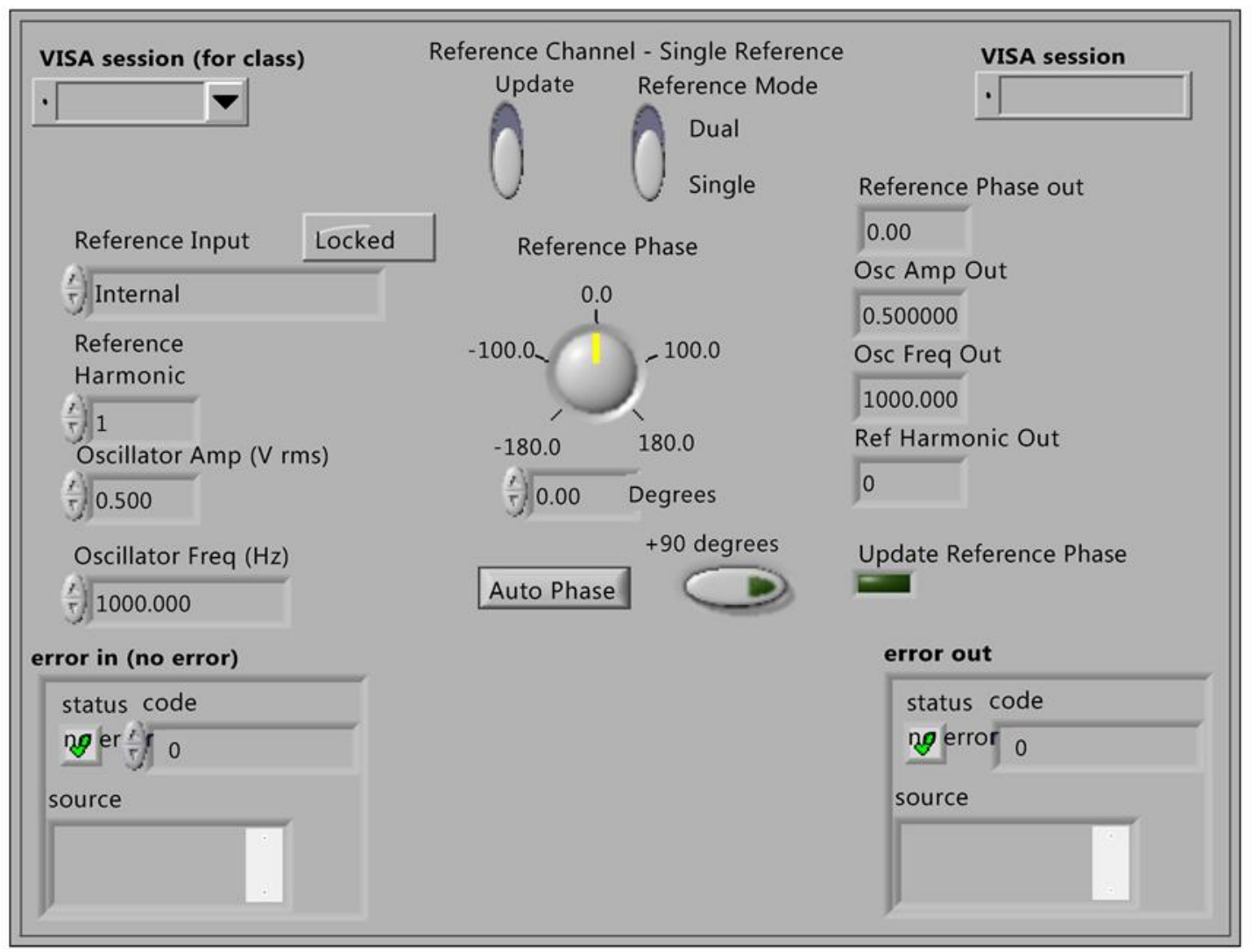


SR7270 Configure Reference Channel - Single Reference.vi

Page $3 \begin{gathered}7270 \\ \text { ref } \\ \text { chan }\end{gathered}$

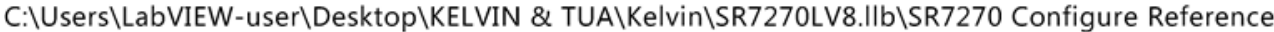

Channel - Single Reference.vi

Last modified on 5/11/2009 at 6:58 PM

Printed on 5/20/2016 at 8:02 PM

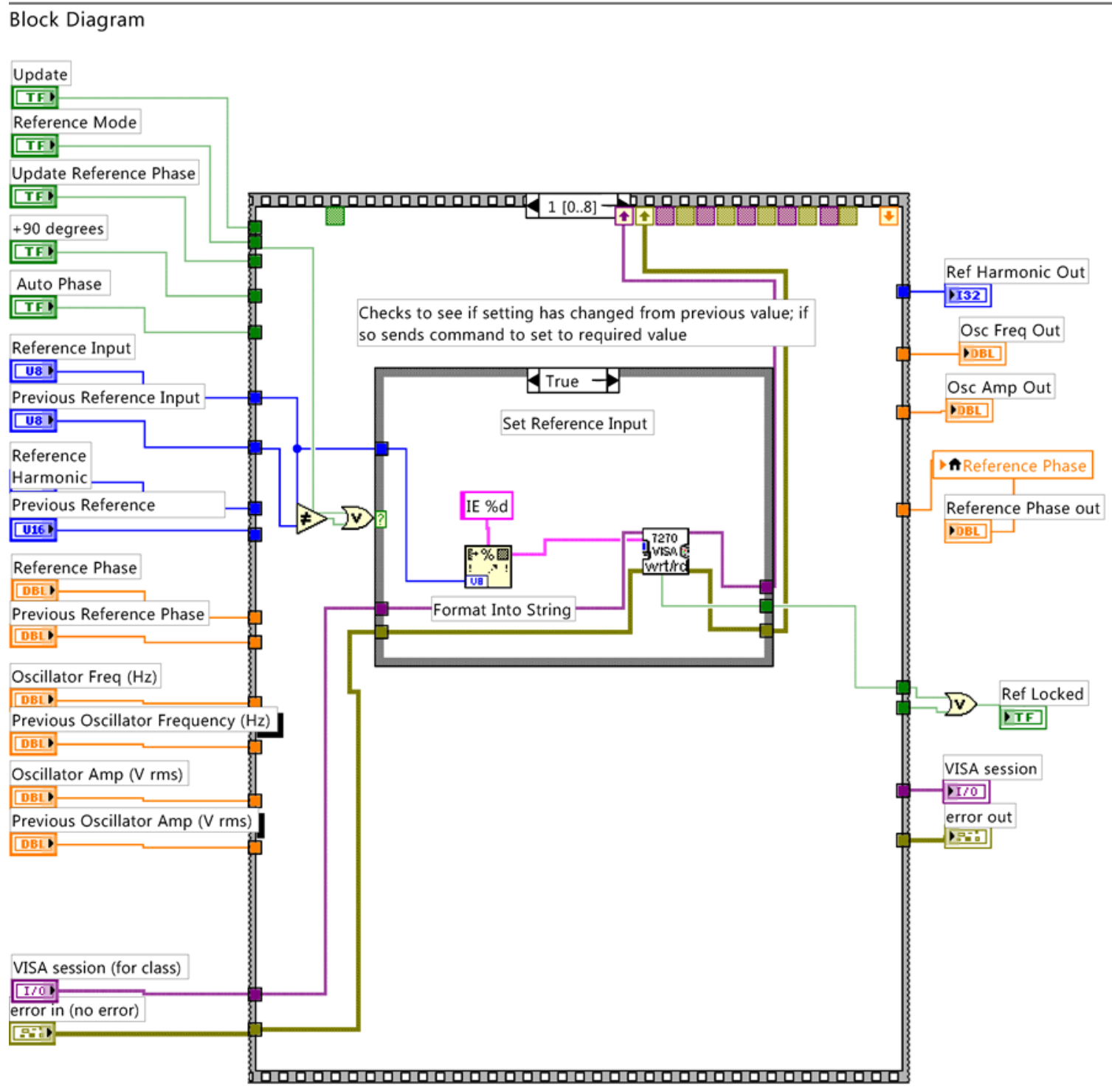


SR7270 Configure Reference Channel - Single Reference.vi

Page $4 \begin{gathered}7270 \\ \text { ref } \\ \text { chan }\end{gathered}$

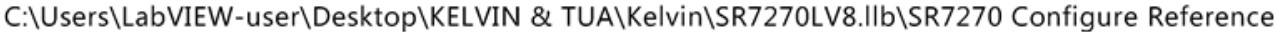

Channel - Single Reference.vi

Last modified on 5/11/2009 at 6:58 PM

Printed on 5/20/2016 at 8:02 PM

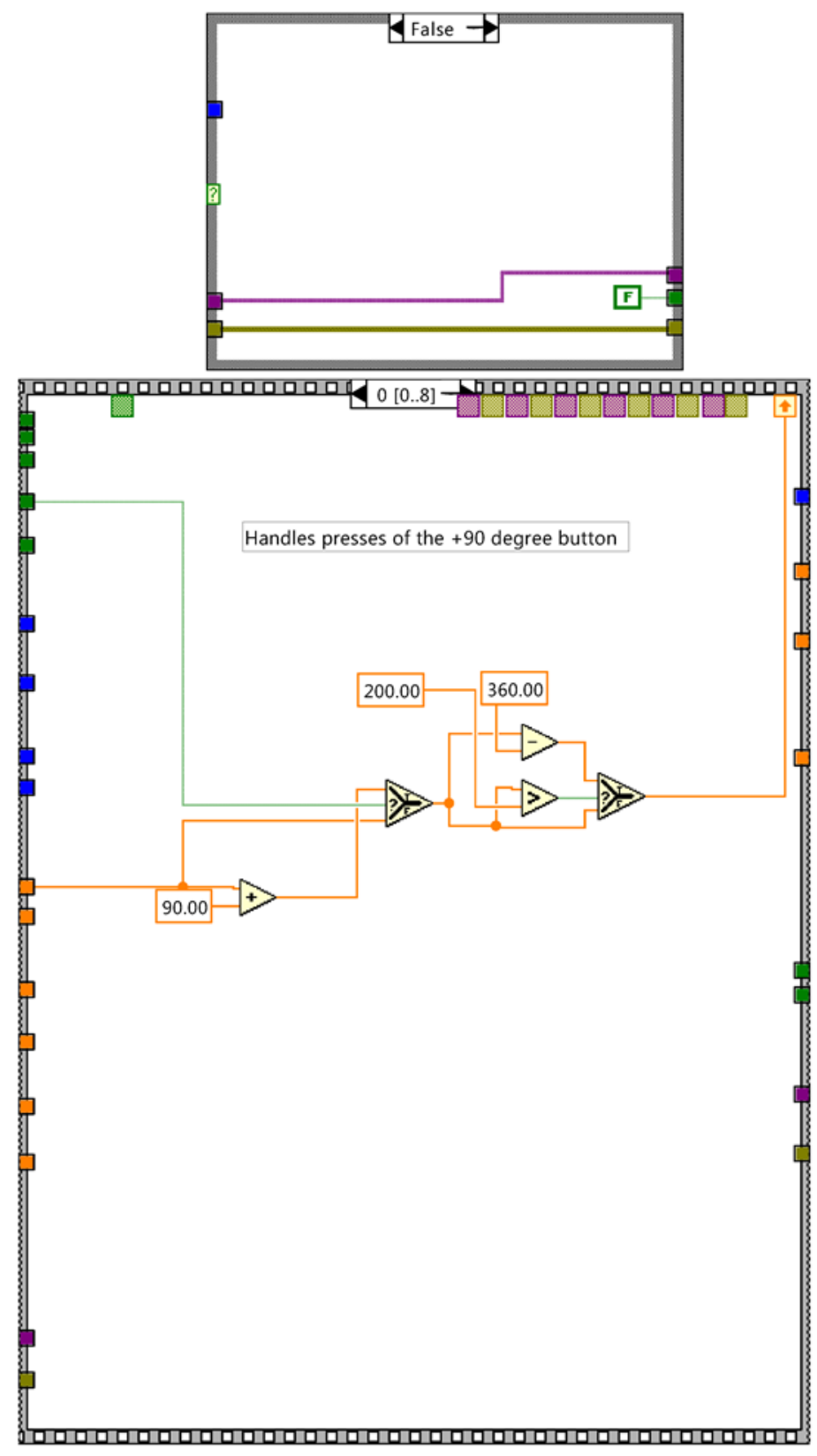


SR7270 Configure Reference Channel - Single Reference.vi

Page $5 \stackrel{\begin{array}{c}7270 \\ \text { ref } \\ \text { chan }\end{array}}{ }$

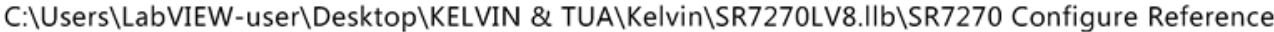

Channel - Single Reference.vi

Last modified on 5/11/2009 at 6:58 PM

Printed on 5/20/2016 at 8:02 PM

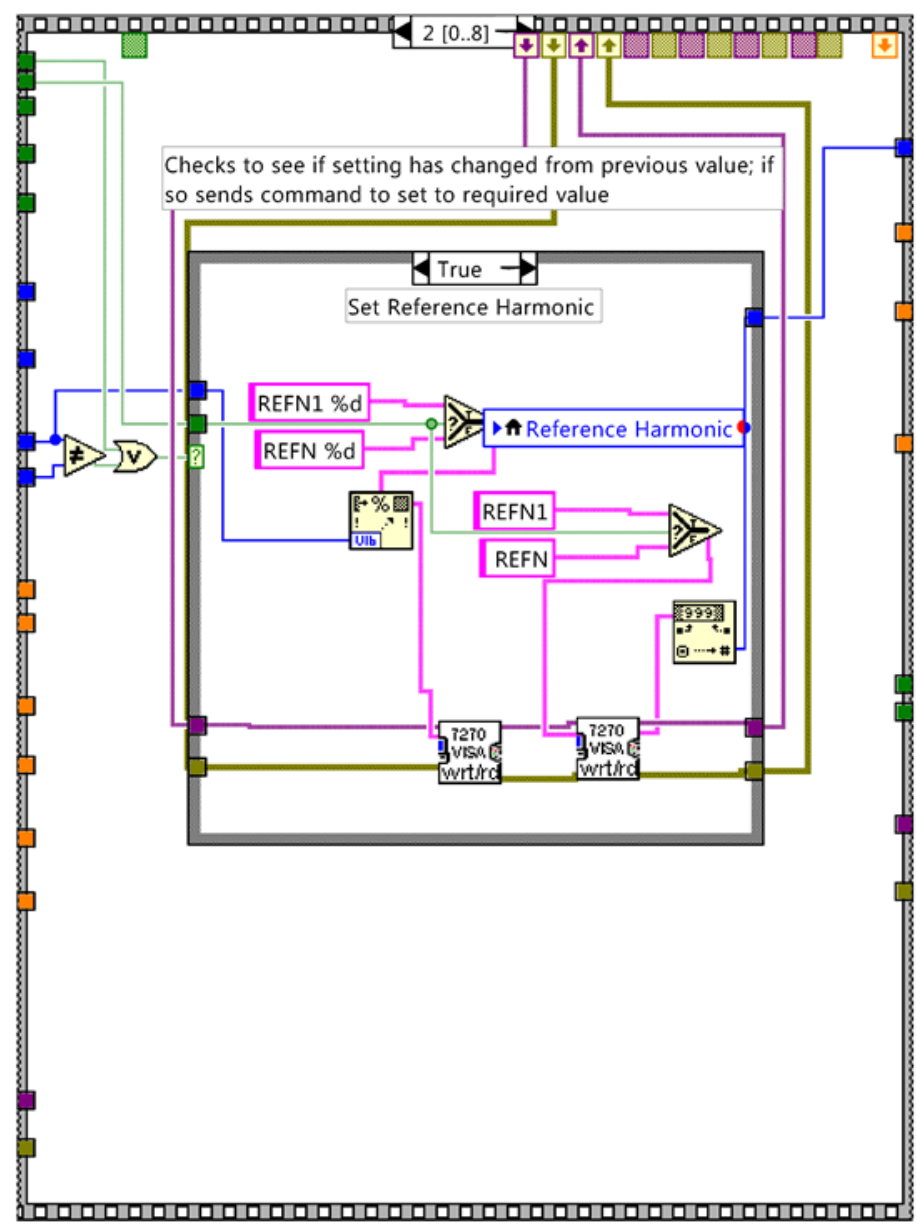


SR7270 Configure Reference Channel - Single Reference.vi

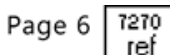

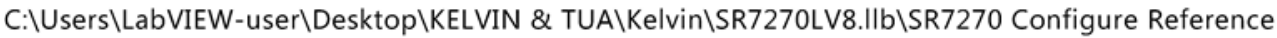

chan

Channel - Single Reference.vi

Last modified on 5/11/2009 at 6:58 PM

Printed on 5/20/2016 at 8:02 PM

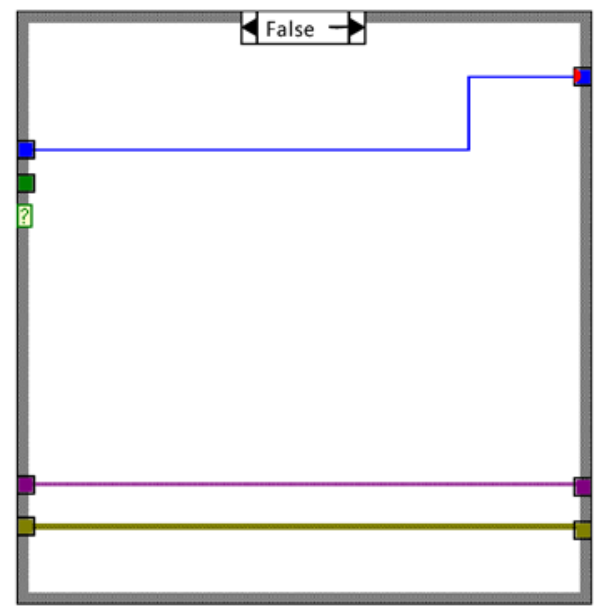


SR7270 Configure Reference Channel - Single Reference.vi

Page $7 \begin{gathered}7270 \\ \text { ref } \\ \text { chan }\end{gathered}$

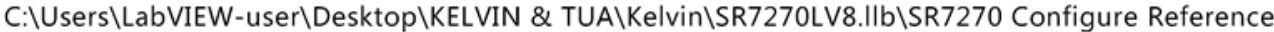

Channel - Single Reference.vi

Last modified on 5/11/2009 at 6:58 PM

Printed on 5/20/2016 at 8:02 PM
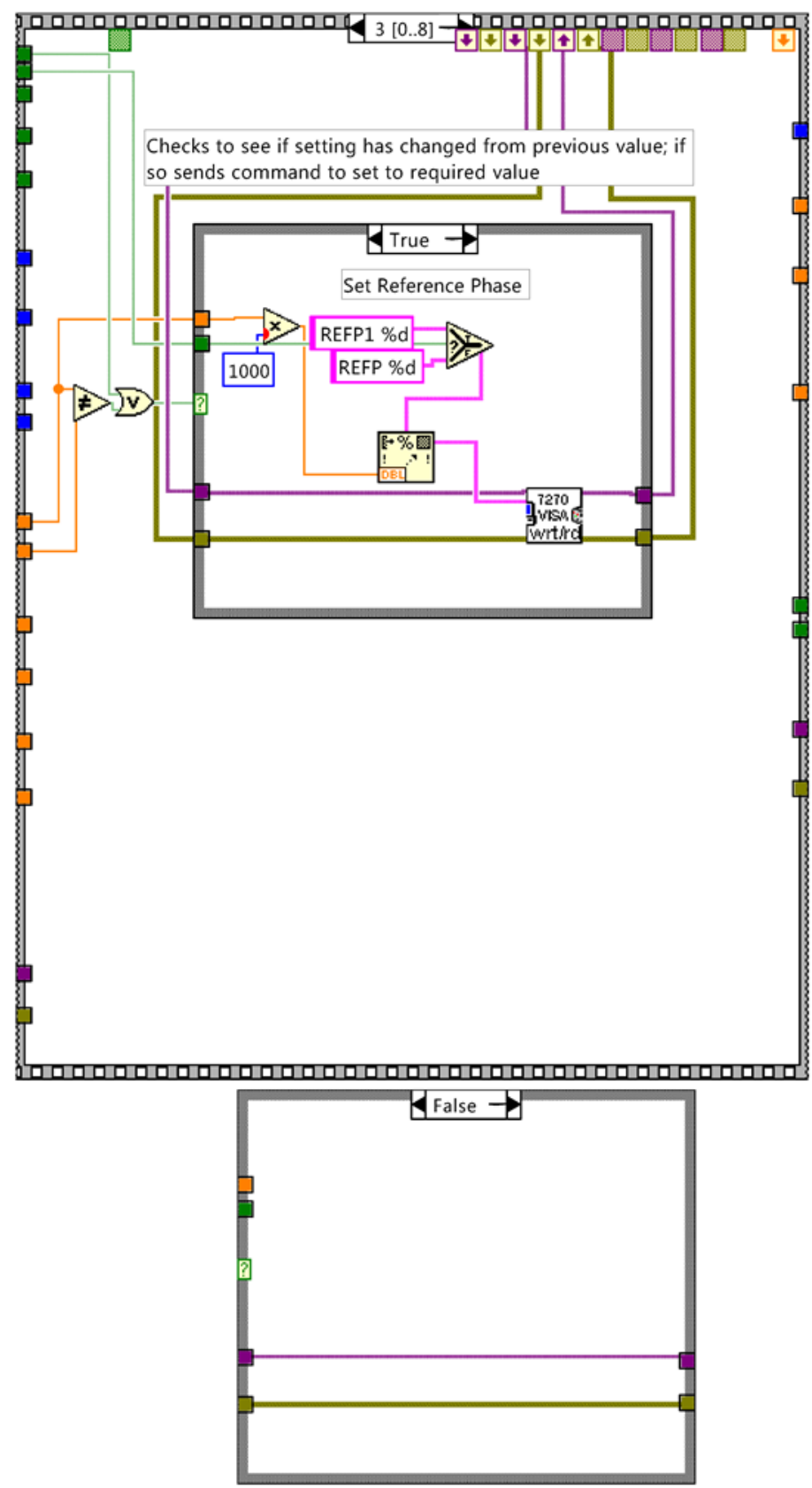
SR7270 Configure Reference Channel - Single Reference.vi

Page $8 \stackrel{\begin{array}{c}7270 \\ \text { ref } \\ \text { chan }\end{array}}{ }$

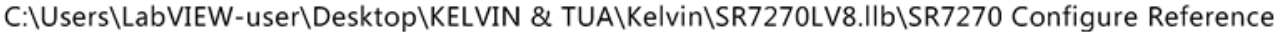

Channel - Single Reference.vi

Last modified on 5/11/2009 at 6:58 PM

Printed on 5/20/2016 at 8:02 PM
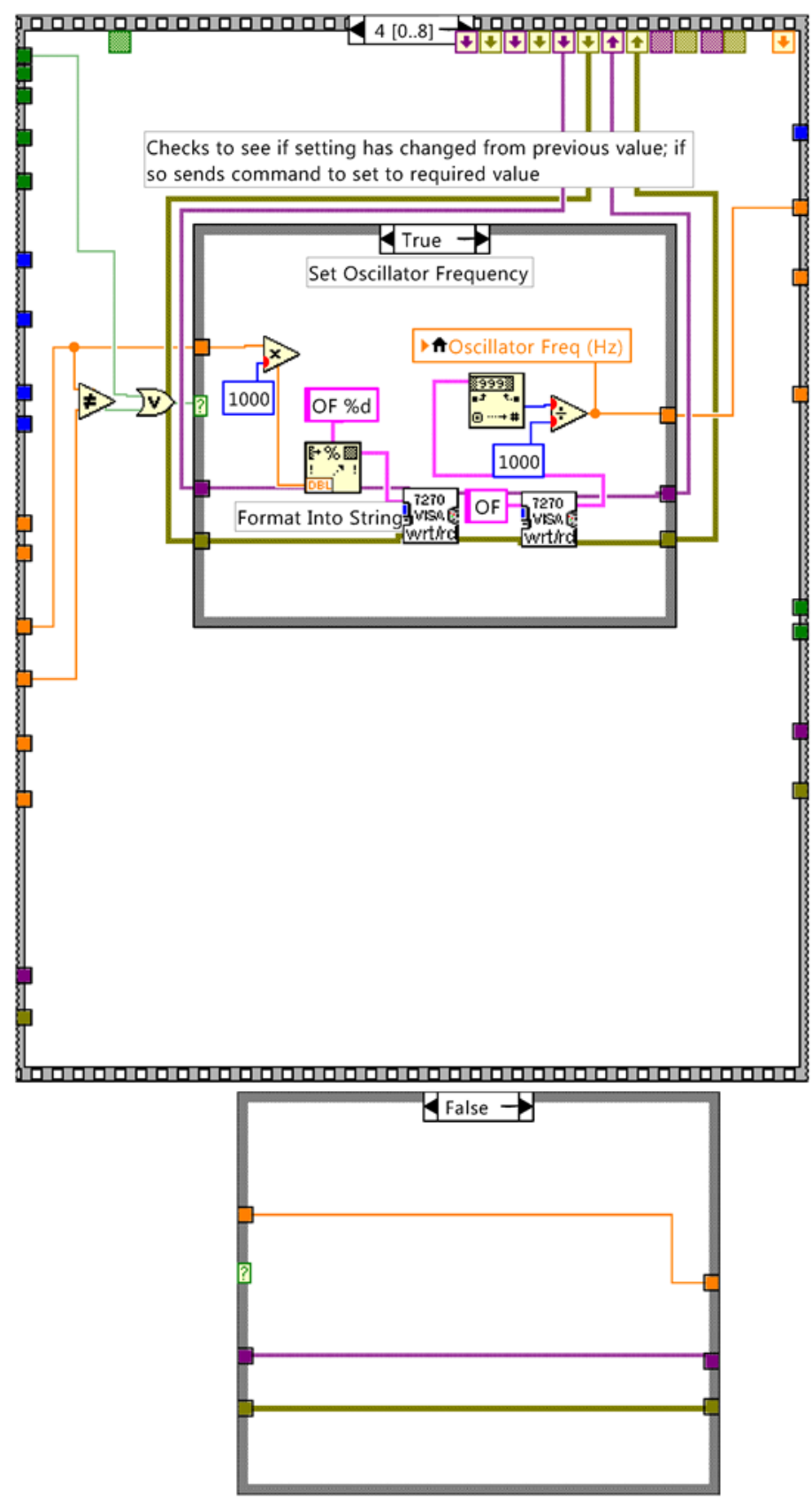
SR7270 Configure Reference Channel - Single Reference.vi

Page $9 \begin{gathered}7270 \\ \text { ref } \\ \text { chan }\end{gathered}$

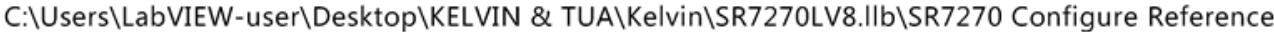

Channel - Single Reference.vi

Last modified on 5/11/2009 at 6:58 PM

Printed on 5/20/2016 at 8:02 PM
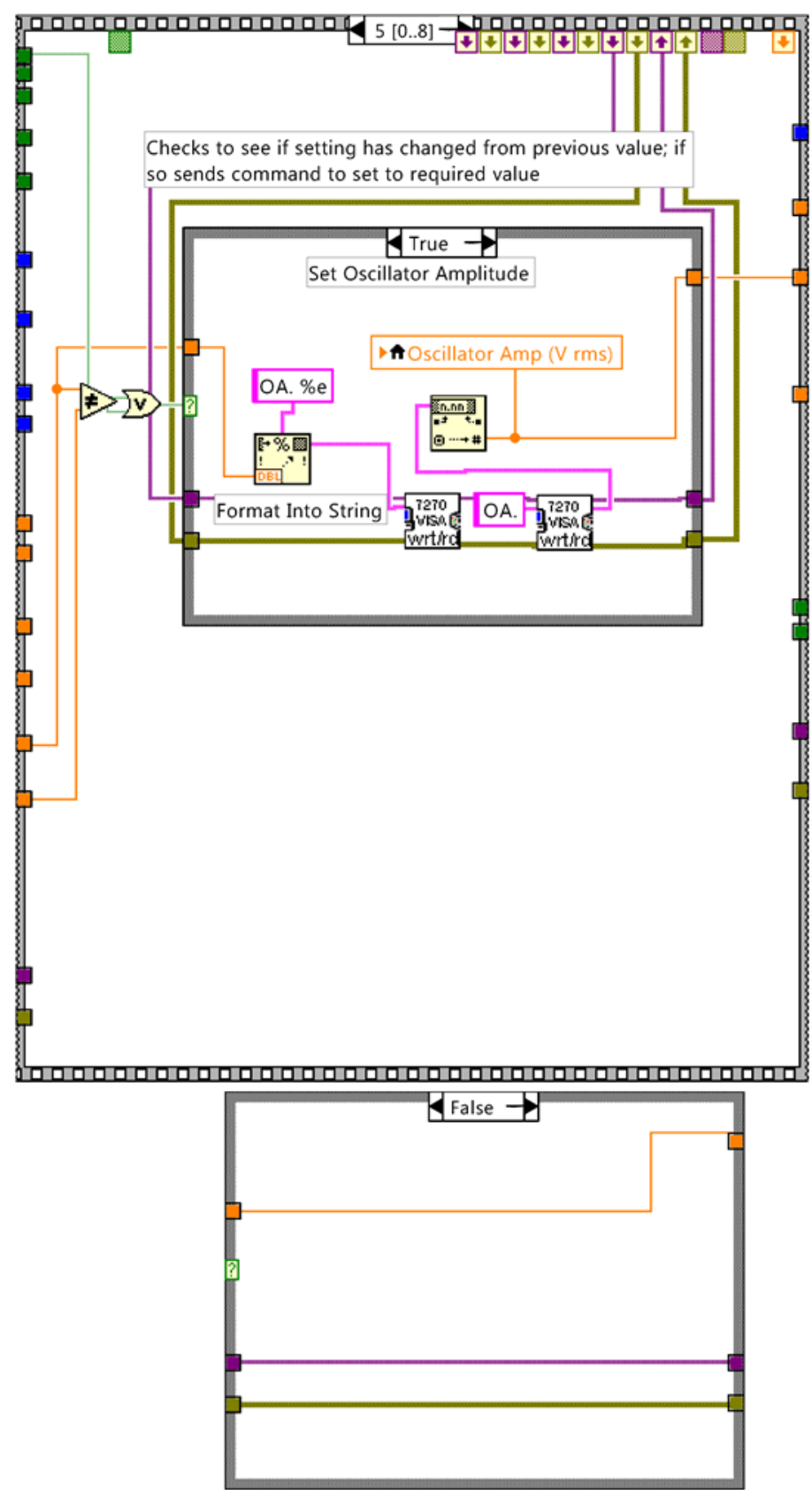
SR7270 Configure Reference Channel - Single Reference.vi

Page $10 \stackrel{\begin{array}{c}7270 \\ \text { ref } \\ \text { chan }\end{array}}{ }$

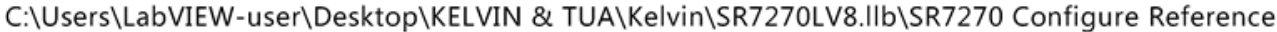

Channel - Single Reference.vi

Last modified on 5/11/2009 at 6:58 PM

Printed on 5/20/2016 at 8:02 PM
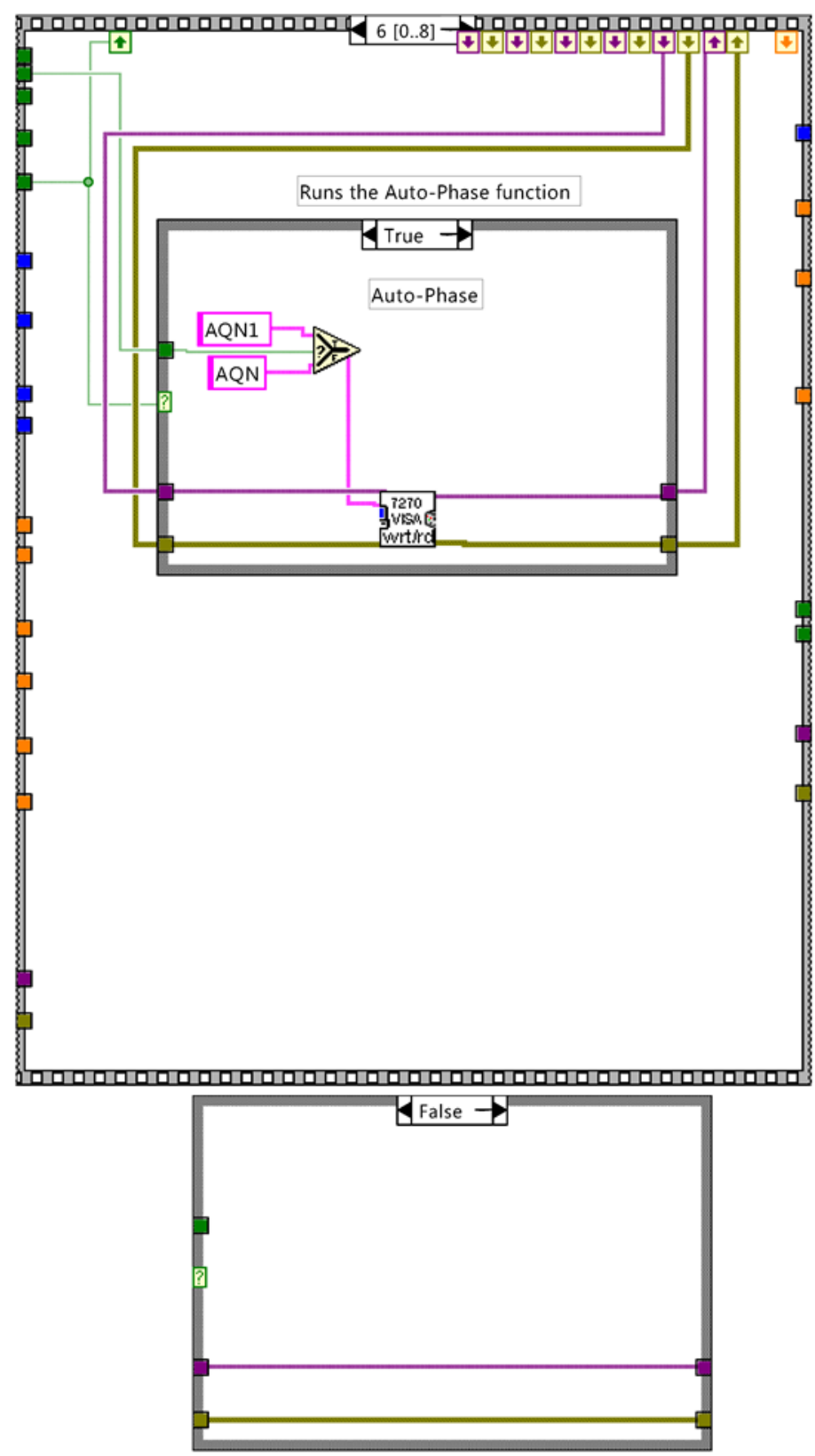
SR7270 Configure Reference Channel - Single Reference.vi

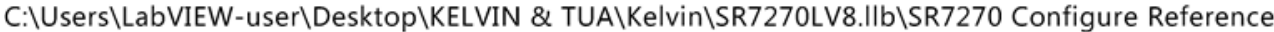

Channel - Single Reference.vi

Last modified on 5/11/2009 at 6:58 PM

Printed on 5/20/2016 at 8:02 PM

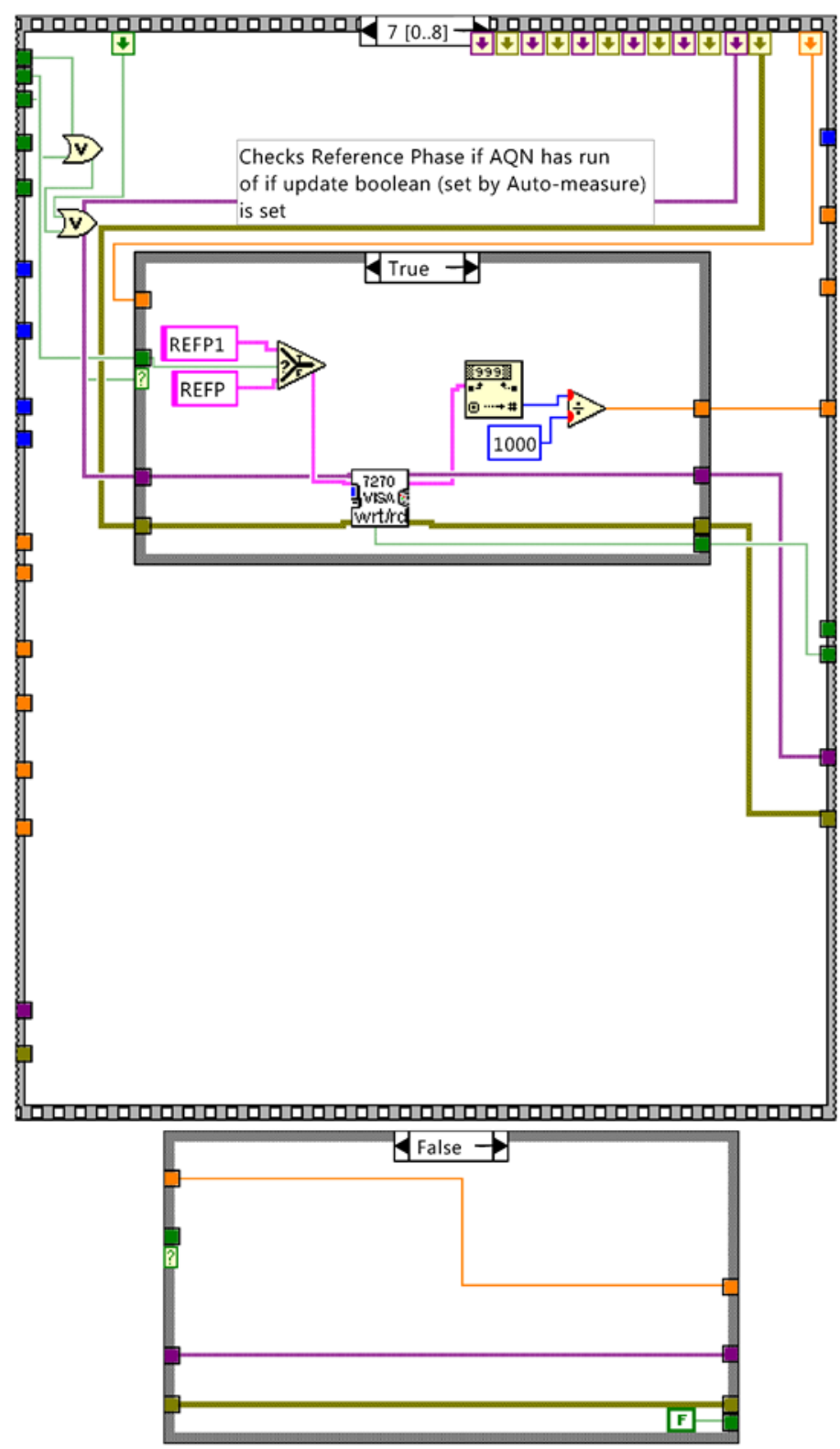


SR7270 Configure Reference Channel - Single Reference.vi

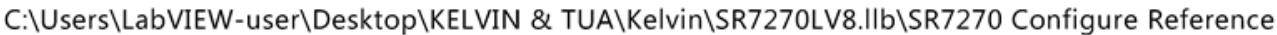

Channel - Single Reference.vi

Last modified on 5/11/2009 at 6:58 PM

Printed on 5/20/2016 at 8:02 PM

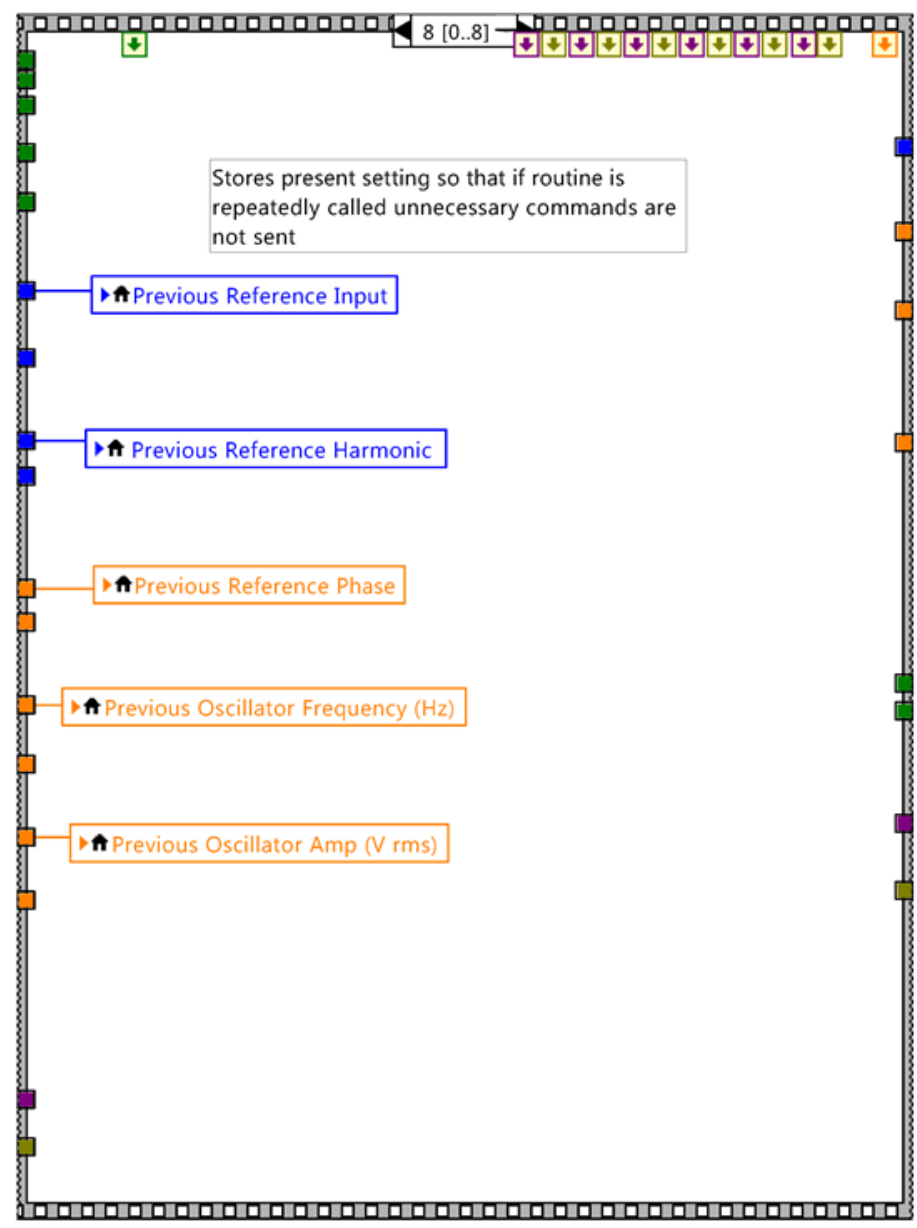


Appendix 32 Cryo3-SubVI: SR7270 Configure Sensitive-Single Reference.vi 
SR7270 Configure Sensitivity - Single Reference.vi

C:IUsers\LabVIEW-user\Desktop\KELVIN \& TUA\Kelvin\SR7270LV8.Ilb\SR7270 Configure Sensitivity -

Single Reference.vi

Last modified on 5/11/2009 at 6:58 PM

Printed on 5/20/2016 at 8:03 PM

Connector Pane

SR7270 Configure Sensitivity - Single Reference.vi

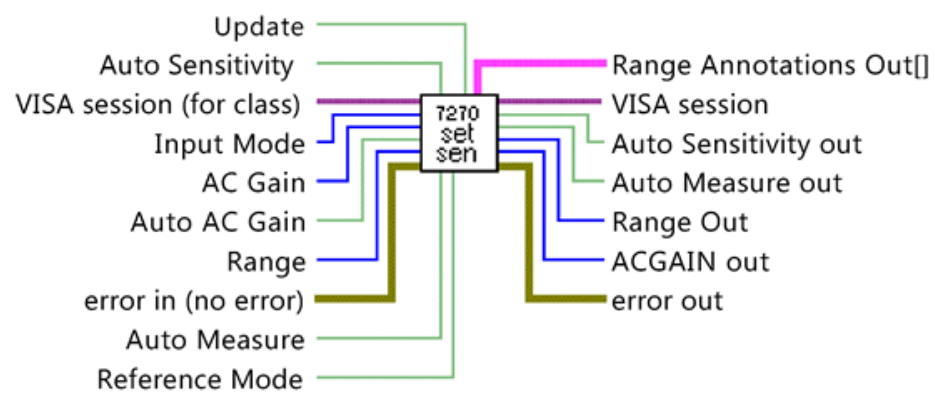

This control sets the full-scale sensitivity of the signal channel, or that of the first channel in the dual reference or harmonic modes. It also sets the AC gain

12 March 2009: First Release 
SR7270 Configure Sensitivity - Single Reference.vi

C:IUsers\LabVIEW-user\Desktop\KELVIN \& TUA\Kelvin\SR7270LV8.Ilb\SR7270 Configure Sensitivity -

Single Reference.vi

Last modified on 5/11/2009 at 6:58 PM

Printed on 5/20/2016 at 8:03 PM

\section{Front Panel}

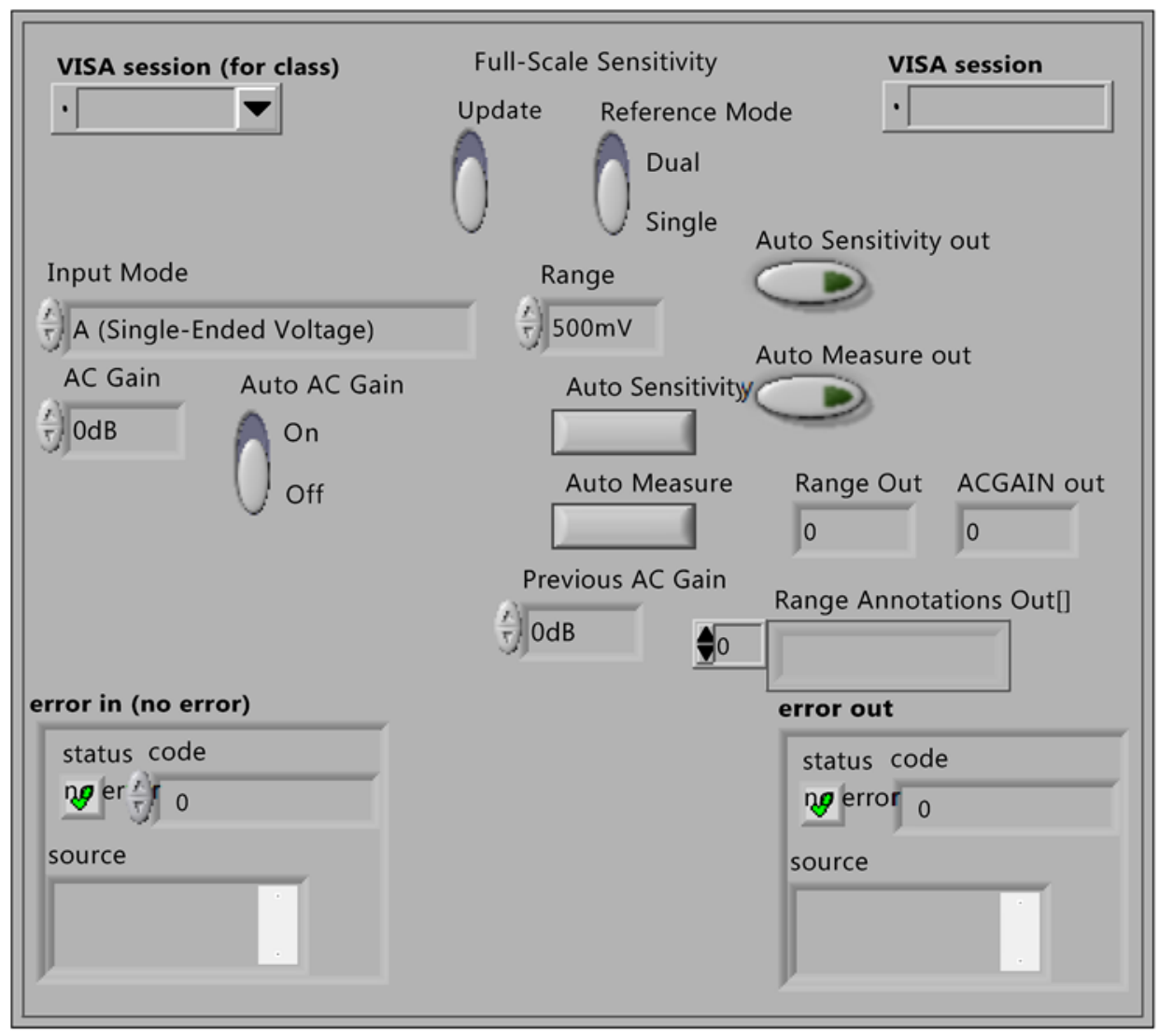


SR7270 Configure Sensitivity - Single Reference.vi

C:IUsers\LabVIEW-user\Desktop\KELVIN \& TUA\Kelvin\SR7270LV8.Ilb\SR7270 Configure Sensitivity -

Single Reference.vi

Last modified on 5/11/2009 at 6:58 PM

Printed on 5/20/2016 at 8:03 PM

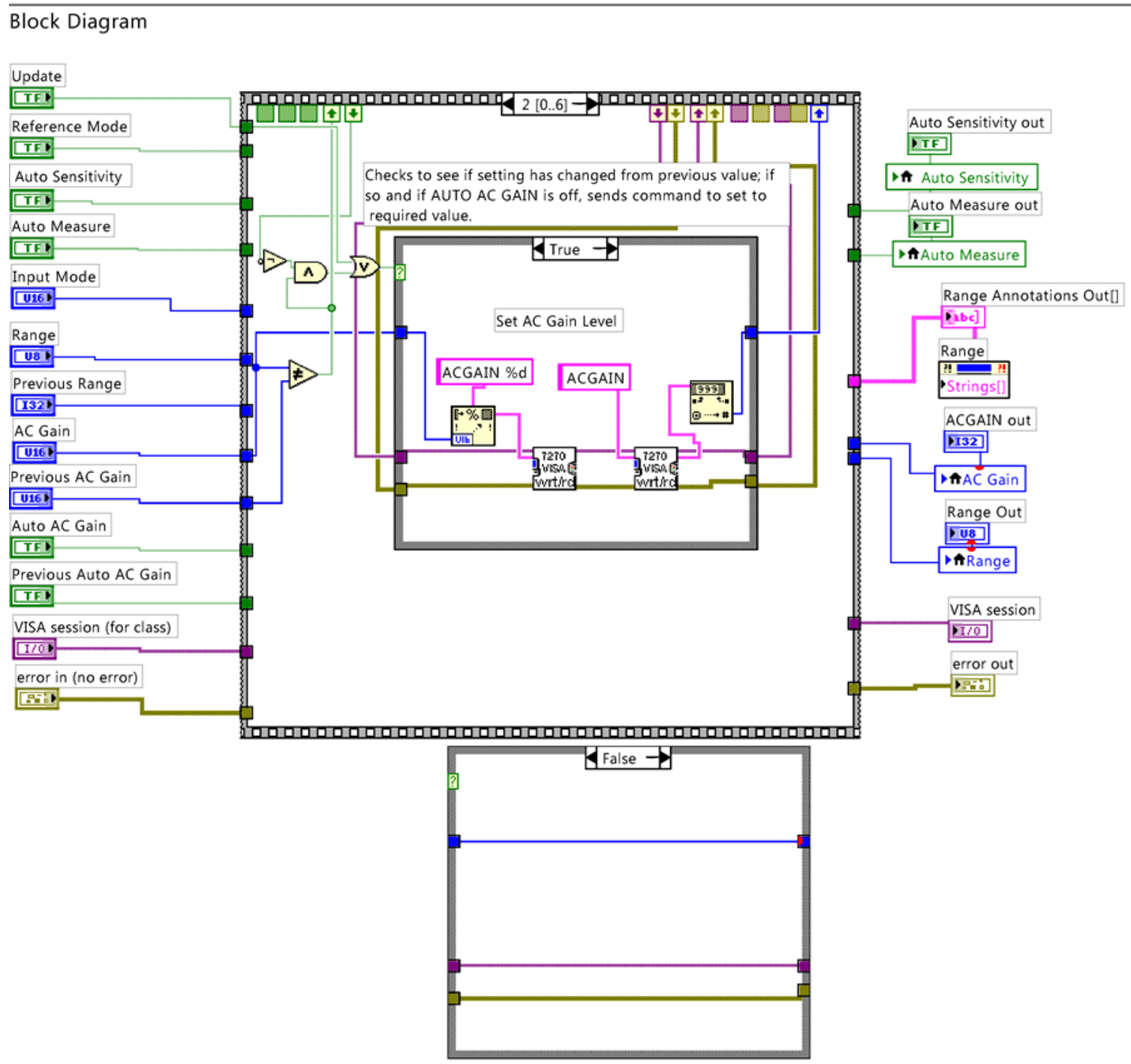


SR7270 Configure Sensitivity - Single Reference.vi

C:IUsers\LabVIEW-user\Desktop\KELVIN \& TUA\Kelvin\SR7270LV8.Ilb\SR7270 Configure Sensitivity Single Reference.vi

Last modified on 5/11/2009 at 6:58 PM

Printed on 5/20/2016 at 8:03 PM
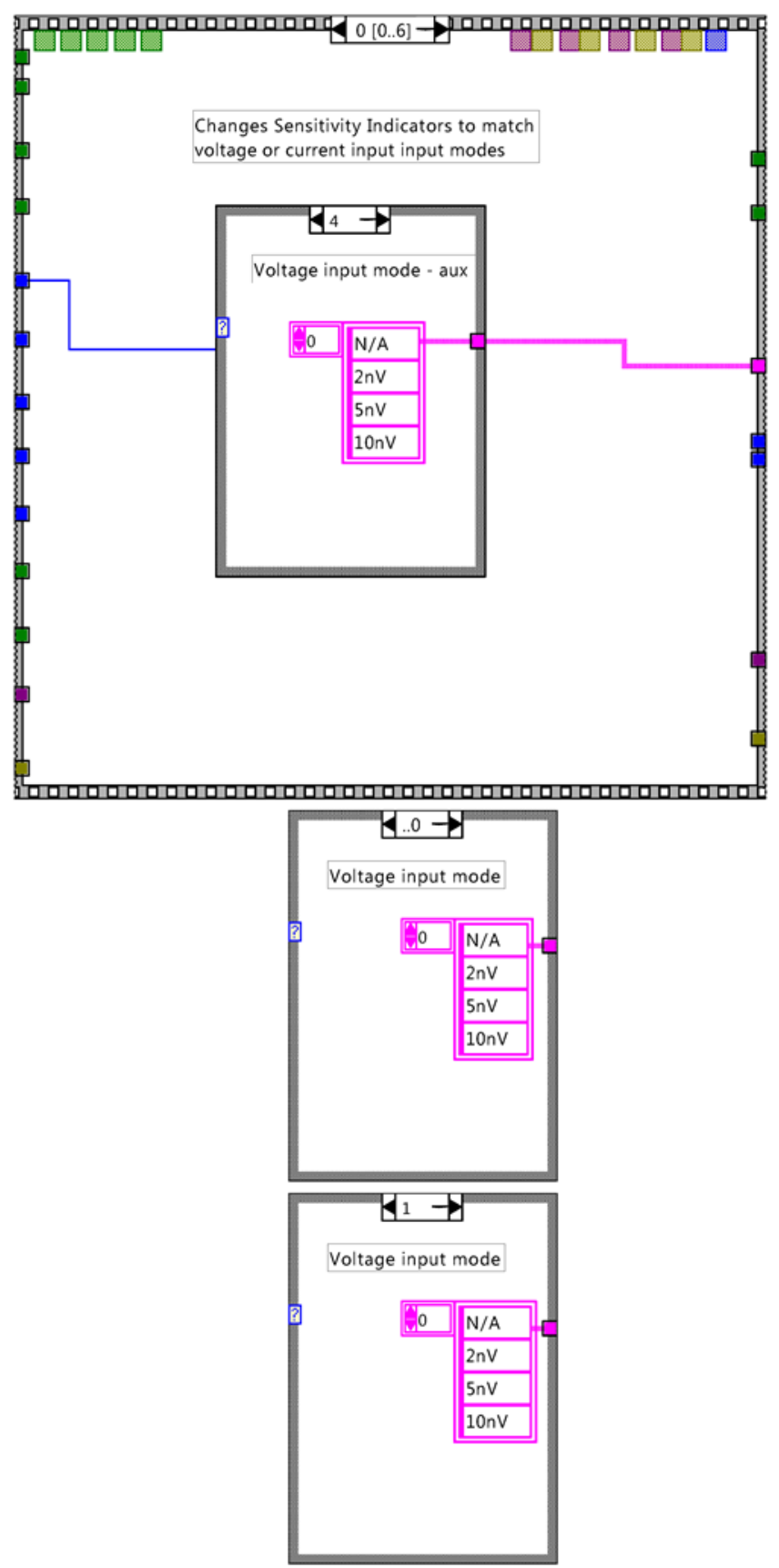
SR7270 Configure Sensitivity - Single Reference.vi

C:IUsers\LabVIEW-user\Desktop\KELVIN \& TUA\Kelvin\SR7270LV8.Ilb\SR7270 Configure Sensitivity Single Reference.vi

Last modified on 5/11/2009 at 6:58 PM

Printed on 5/20/2016 at 8:03 PM
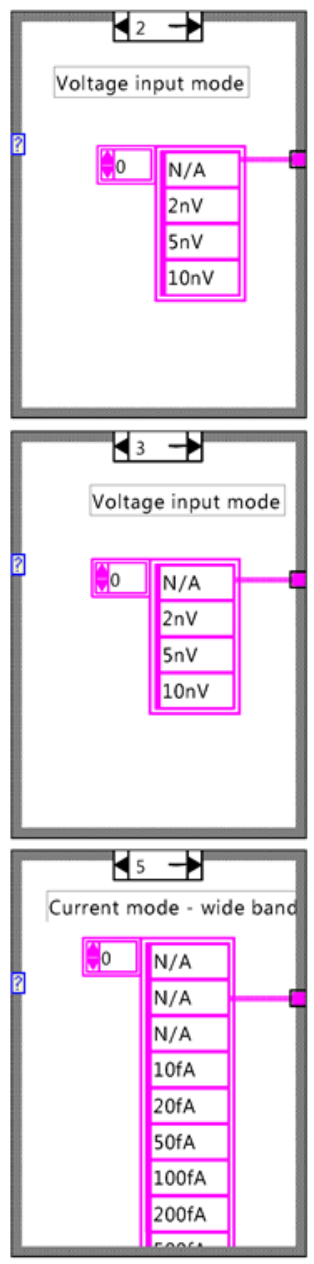

\begin{tabular}{|l||l||}
\hline Current mode - low noise \\
\hline N/A \\
\hline N/A \\
\hline N/A \\
\hline N/A \\
\hline N/A \\
\hline N/A \\
\hline N/A \\
\hline
\end{tabular}


SR7270 Configure Sensitivity - Single Reference.vi

C:IUsers\LabVIEW-user\Desktop\KELVIN \& TUA\Kelvin\SR7270LV8.IIb\SR7270 Configure Sensitivity Single Reference.vi

Last modified on 5/11/2009 at 6:58 PM

Printed on 5/20/2016 at 8:03 PM
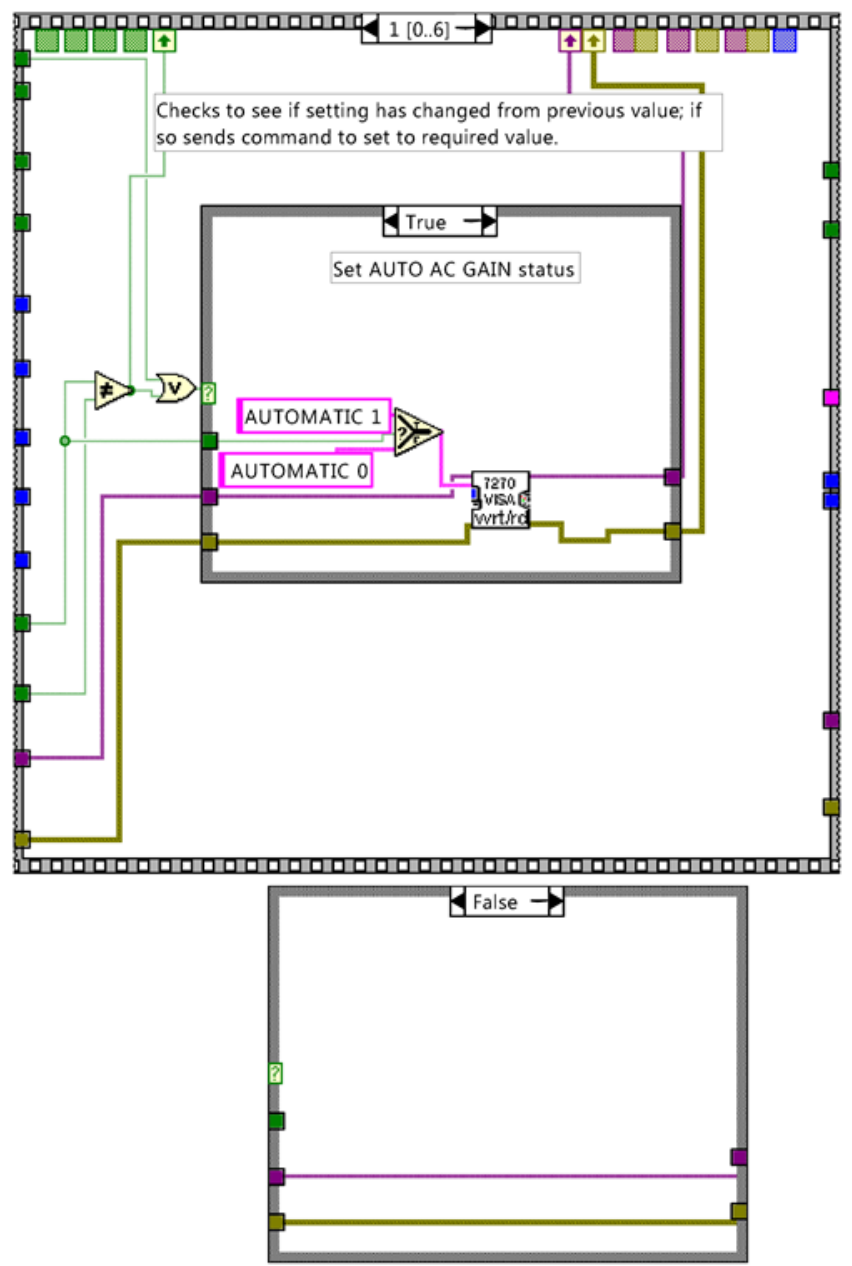
SR7270 Configure Sensitivity - Single Reference.vi

C:IUsers\LabVIEW-user\Desktop\KELVIN \& TUA\Kelvin\SR7270LV8.Ilb\SR7270 Configure Sensitivity Single Reference.vi

Last modified on 5/11/2009 at 6:58 PM

Printed on 5/20/2016 at 8:03 PM
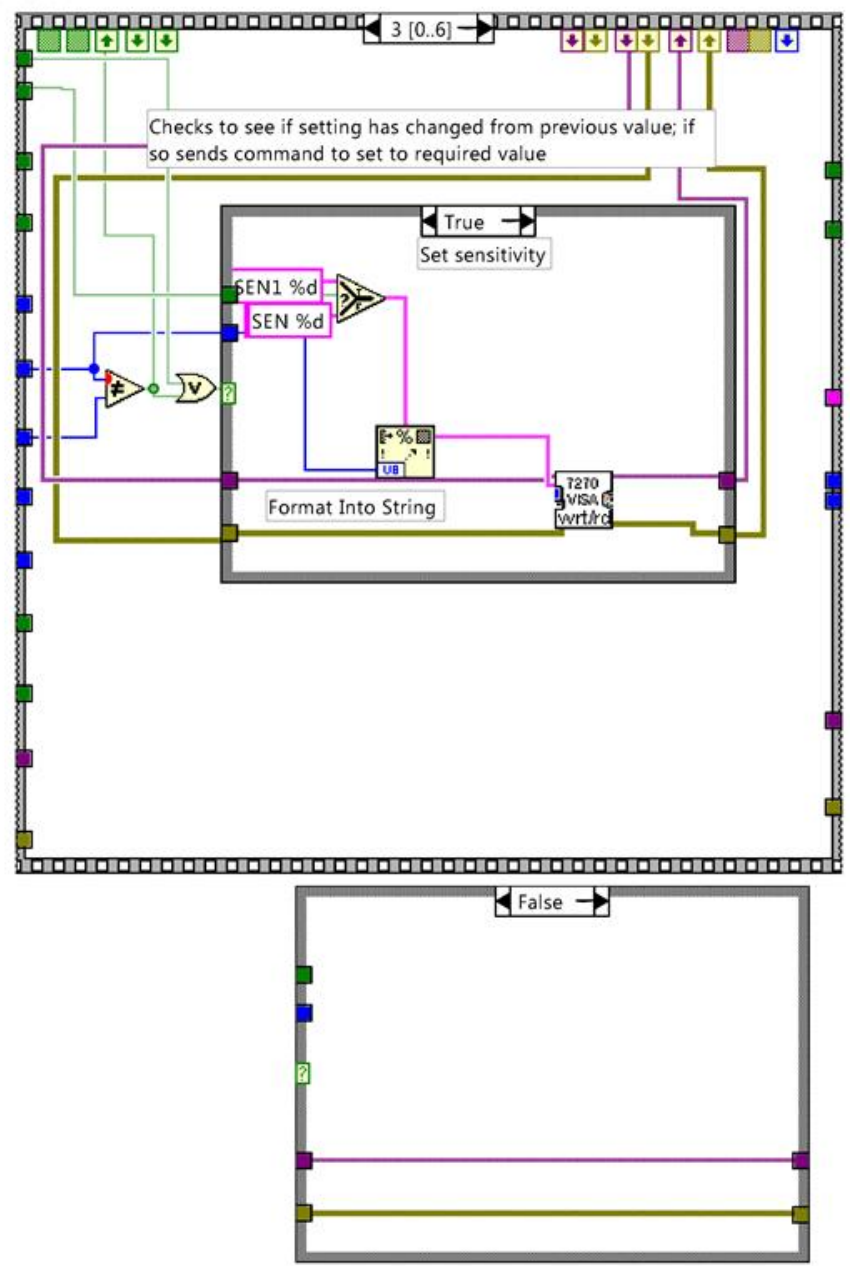
SR7270 Configure Sensitivity - Single Reference.vi

C:IUsers\LabVIEW-user\Desktop\KELVIN \& TUA\Kelvin\SR7270LV8.IIb\SR7270 Configure Sensitivity Single Reference.vi

Last modified on 5/11/2009 at 6:58 PM

Printed on 5/20/2016 at 8:03 PM

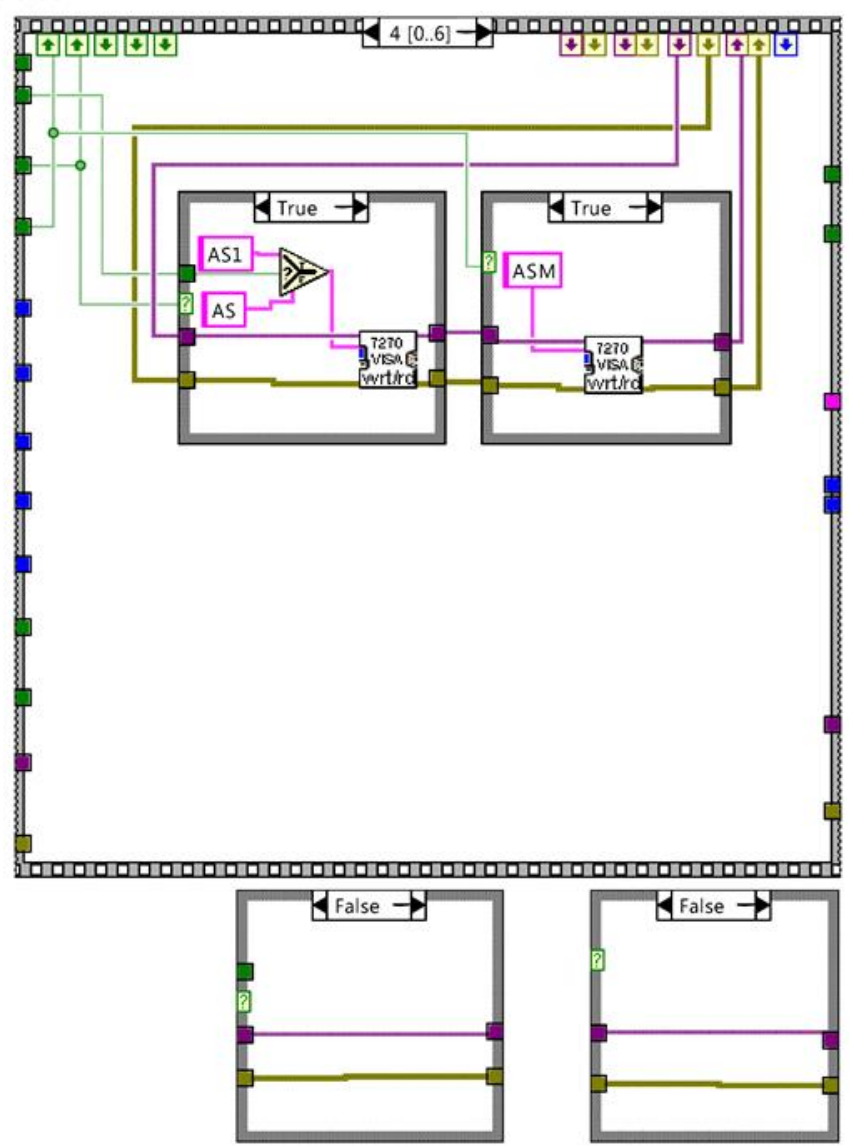


SR7270 Configure Sensitivity - Single Reference.vi

C:IUsers\LabVIEW-user\Desktop\KELVIN \& TUA\Kelvin\SR7270LV8.Ilb\SR7270 Configure Sensitivity Single Reference.vi

Last modified on 5/11/2009 at 6:58 PM

Printed on 5/20/2016 at 8:03 PM
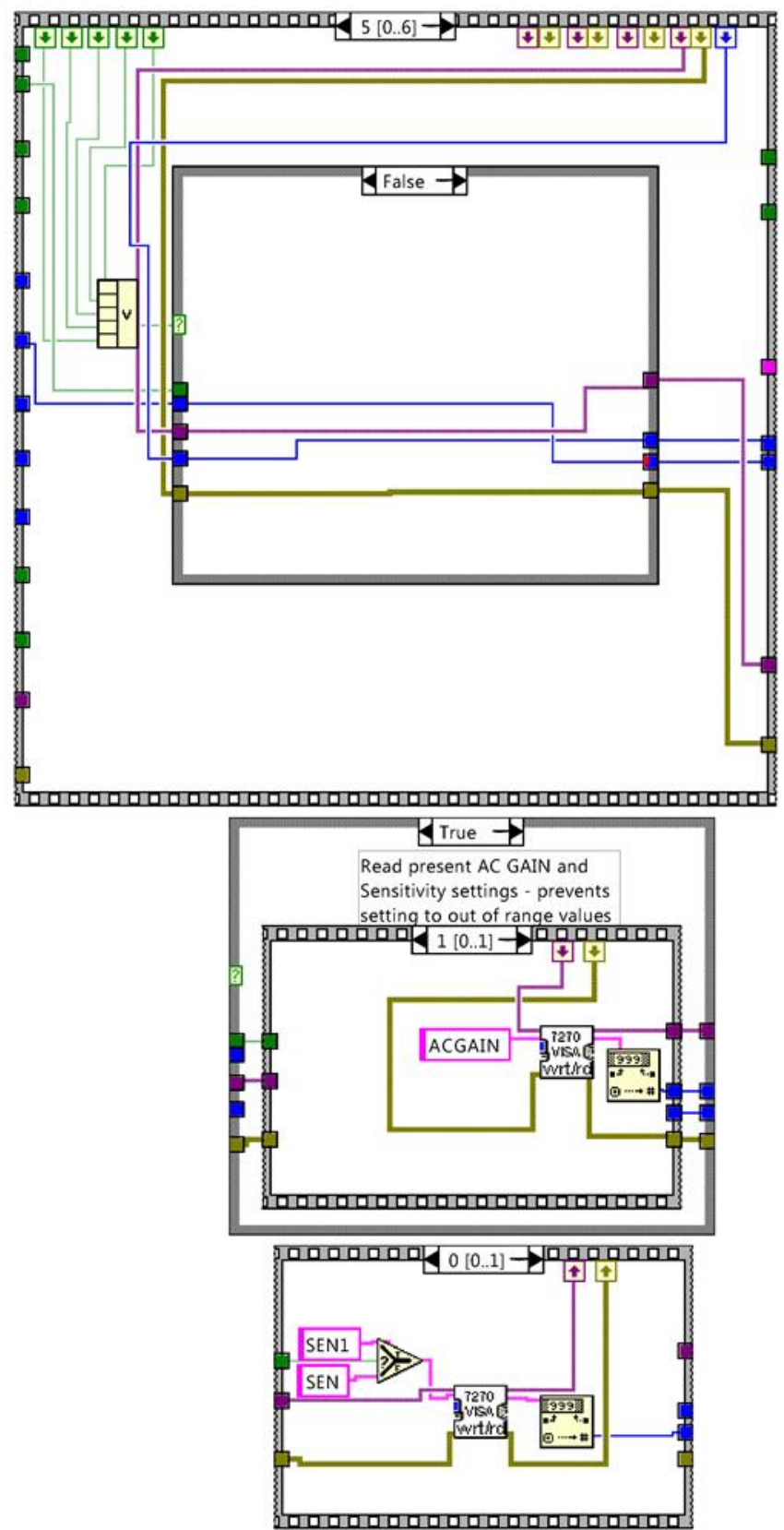
SR7270 Configure Sensitivity - Single Reference.vi

C:IUsers\LabVIEW-user\Desktop\KELVIN \& TUA\Kelvin\SR7270LV8.Ilb\SR7270 Configure Sensitivity -

Single Reference.vi

Last modified on 5/11/2009 at 6:58 PM

Printed on 5/20/2016 at 8:03 PM

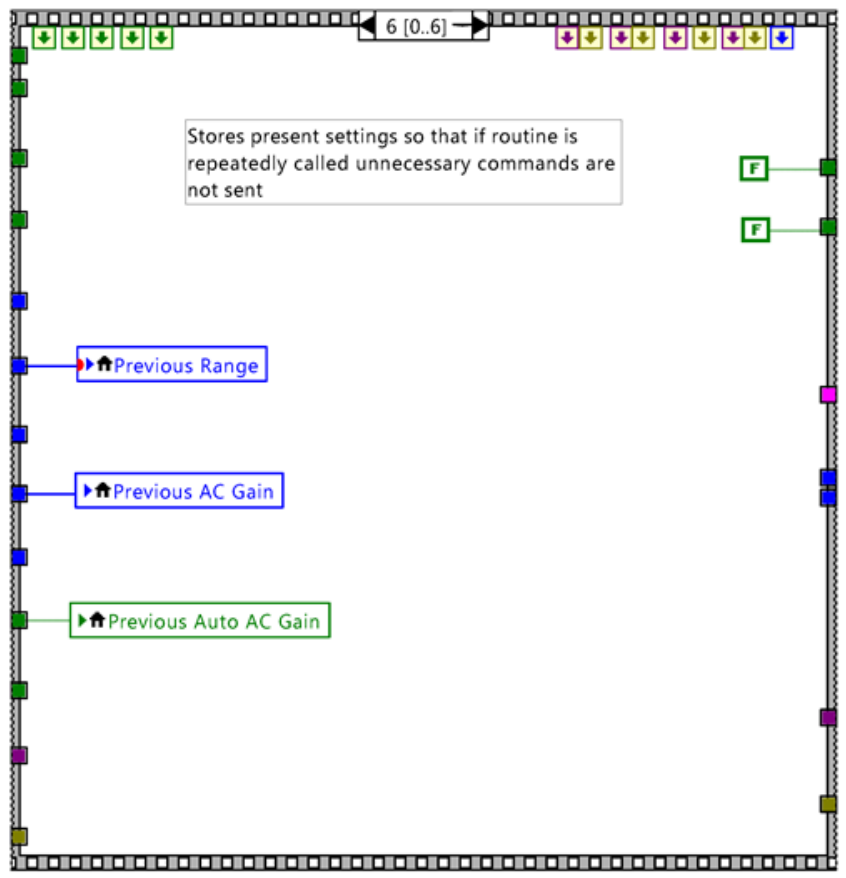


Appendix 33 Cryo3-SubVI: SR7270 Configure Signal Channel Input.vi 
SR7270 Configure Signal Channel Input.vi

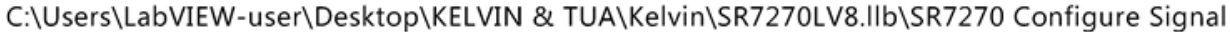

Channel Input.vi

Last modified on 5/11/2009 at 6:57 PM

Printed on 5/20/2016 at 8:04 PM

\section{Connector Pane}

\section{SR7270 Configure Signal Channel Input.vi}

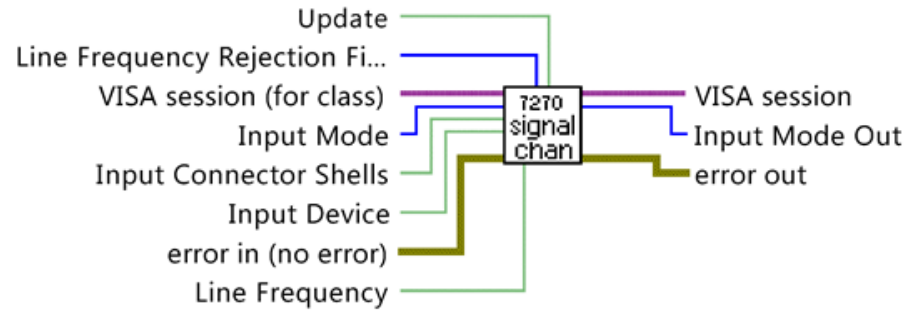

This VI configues the input signal channel and the line-frequency rejection filter.

12 March 2009: First Release

\section{Front Panel}

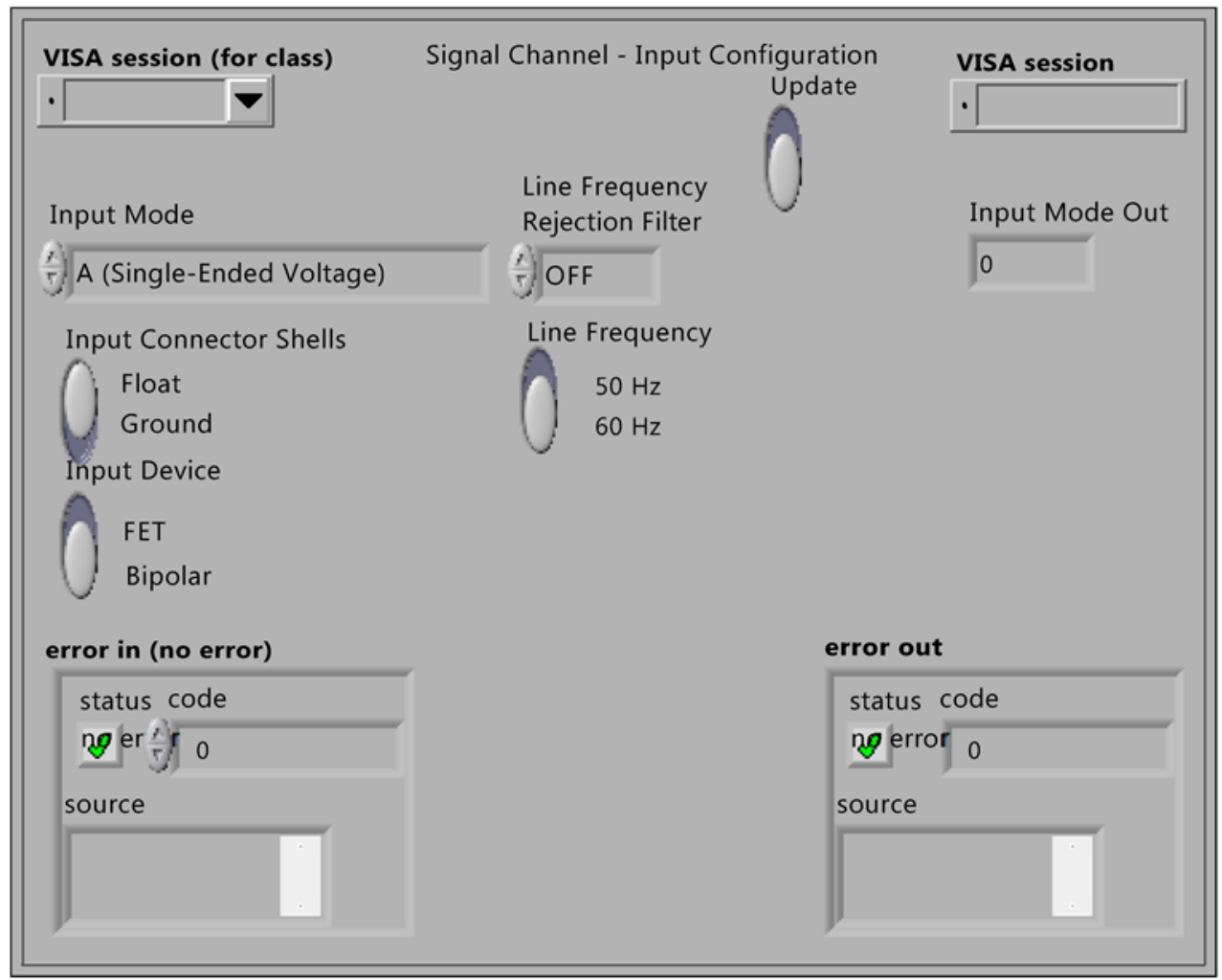


SR7270 Configure Signal Channel Input.vi

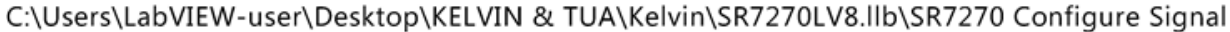

Channel Input.vi

Last modified on 5/11/2009 at 6:57 PM

Printed on 5/20/2016 at 8:04 PM

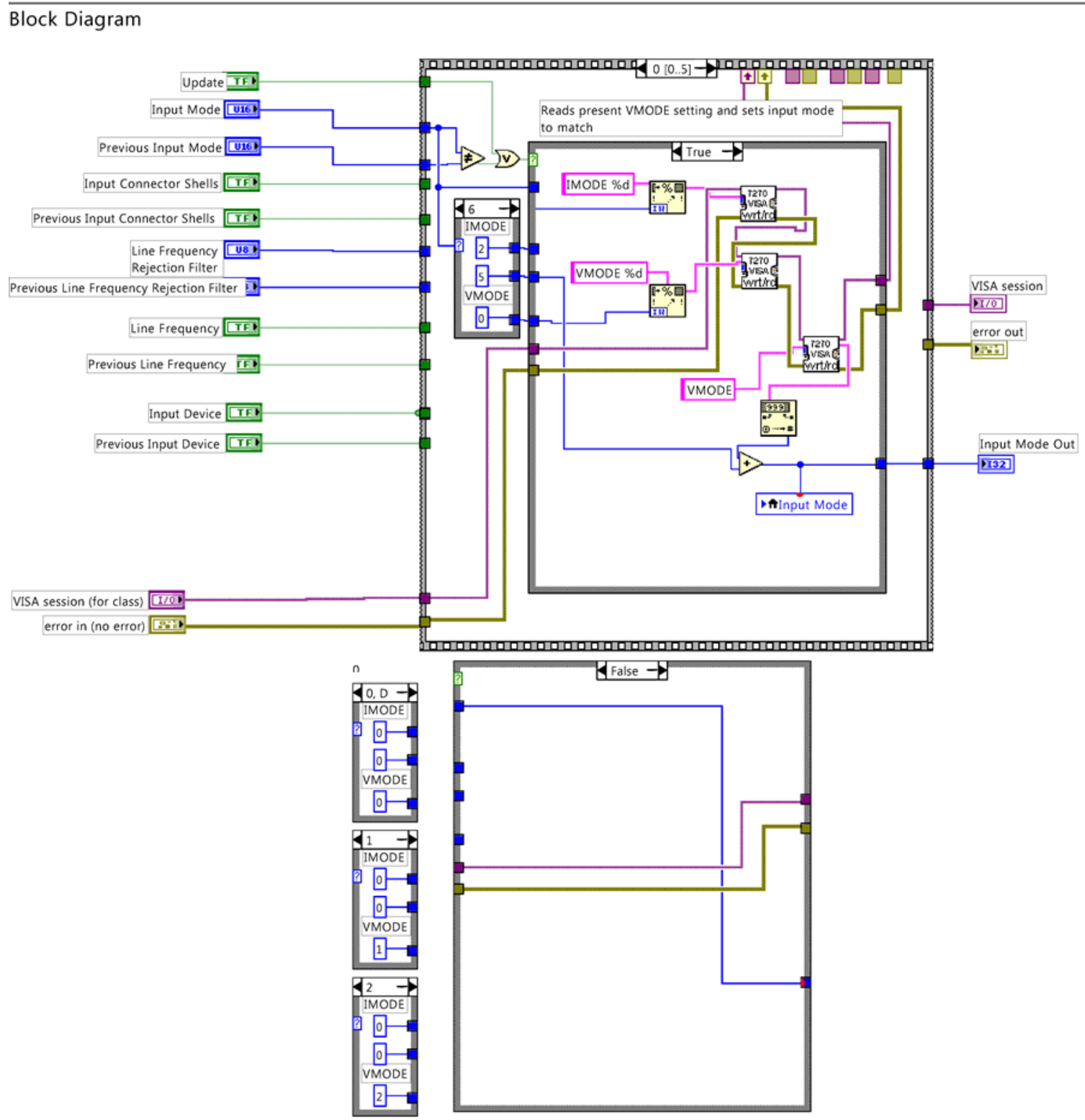


SR7270 Configure Signal Channel Input.vi

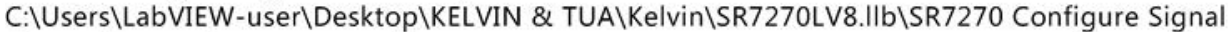
Channel Input.vi

Last modified on 5/11/2009 at 6:57 PM

Printed on 5/20/2016 at 8:04 PM
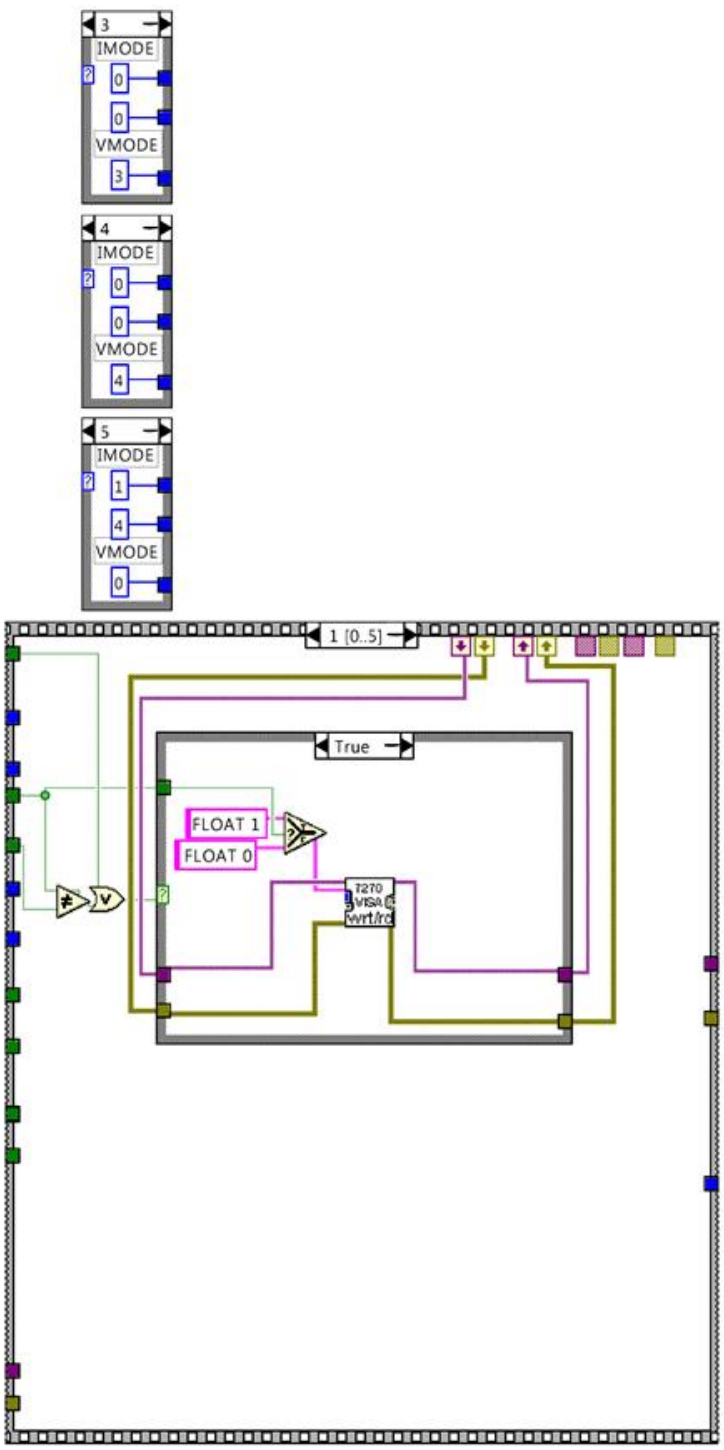
SR7270 Configure Signal Channel Input.vi

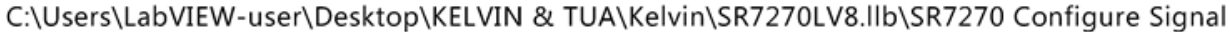

Channel Input.vi

Last modified on 5/11/2009 at 6:57 PM

Printed on 5/20/2016 at 8:04 PM

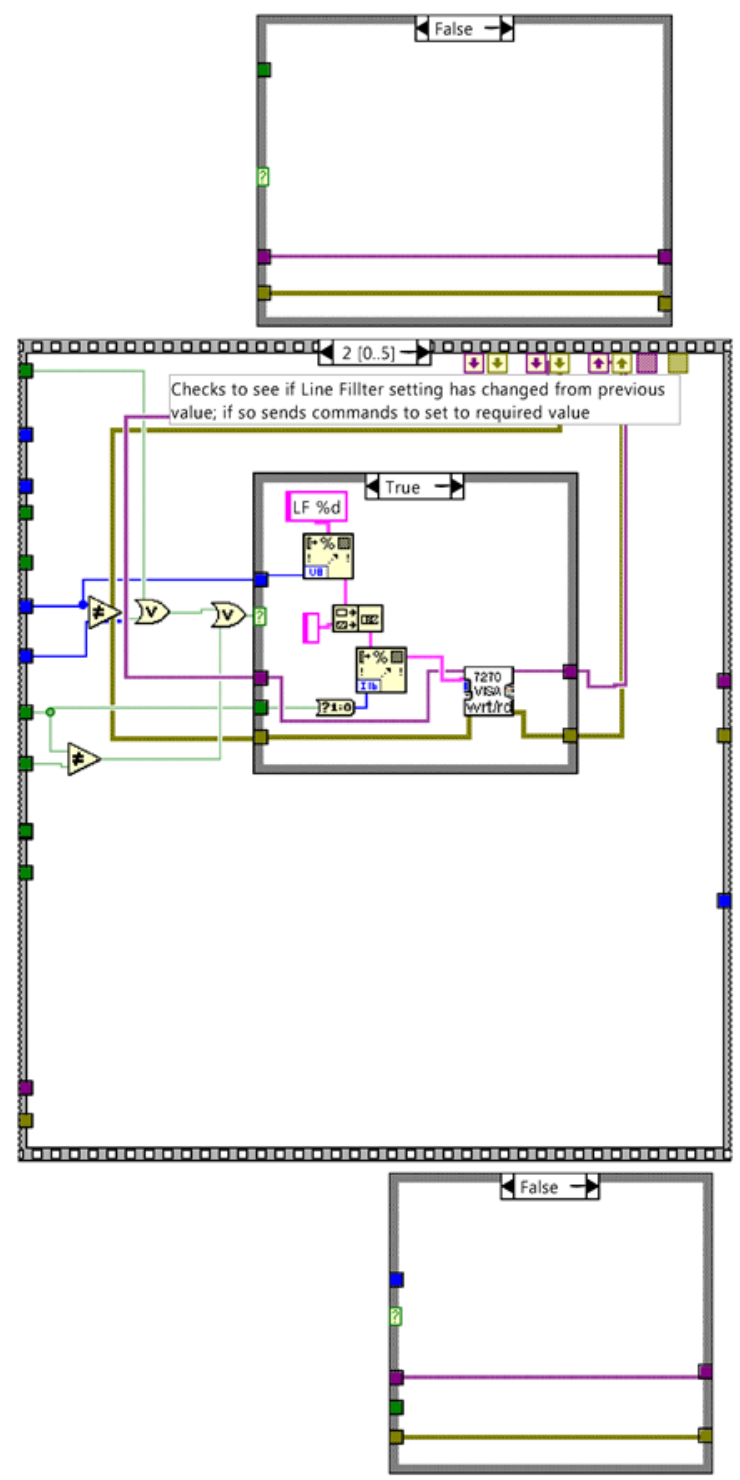


SR7270 Configure Signal Channel Input.vi

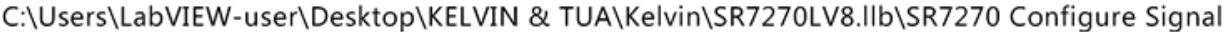

Channel Input.vi

Last modified on 5/11/2009 at 6:57 PM

Printed on 5/20/2016 at 8:04 PM
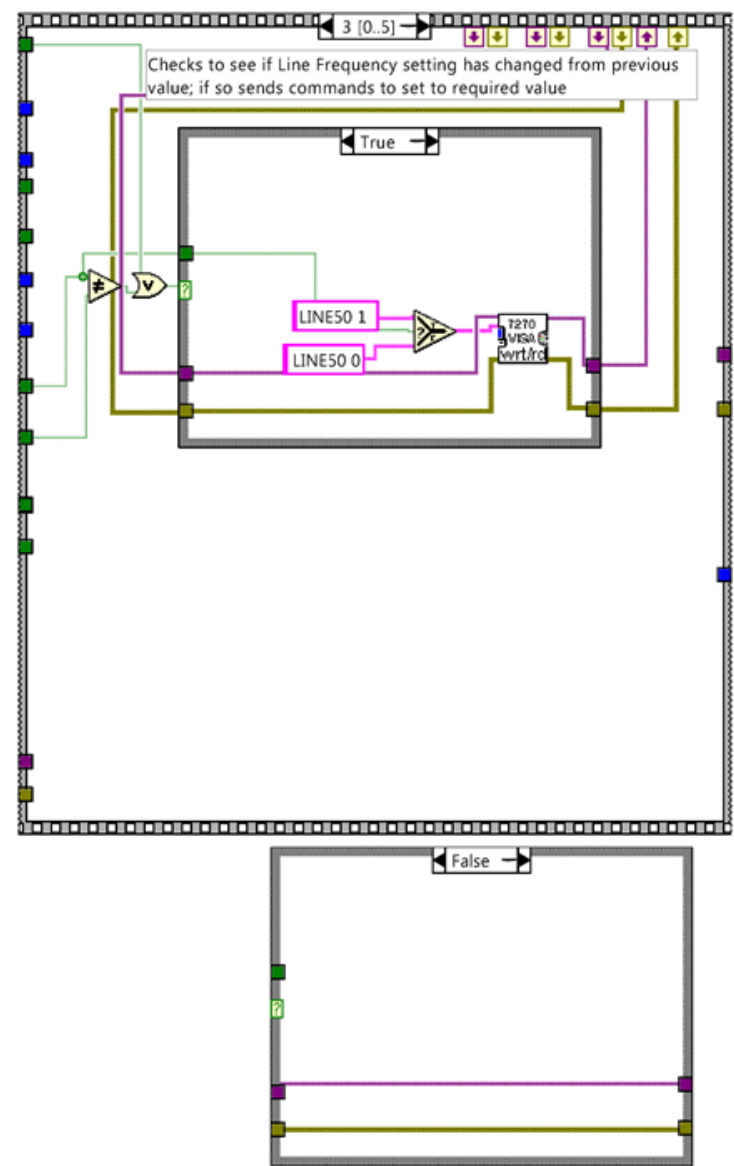
SR7270 Configure Signal Channel Input.vi

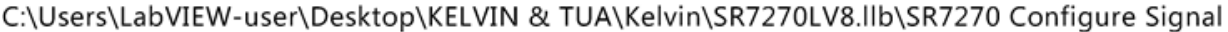

Channel Input.vi

Last modified on 5/11/2009 at 6:57 PM

Printed on 5/20/2016 at 8:04 PM
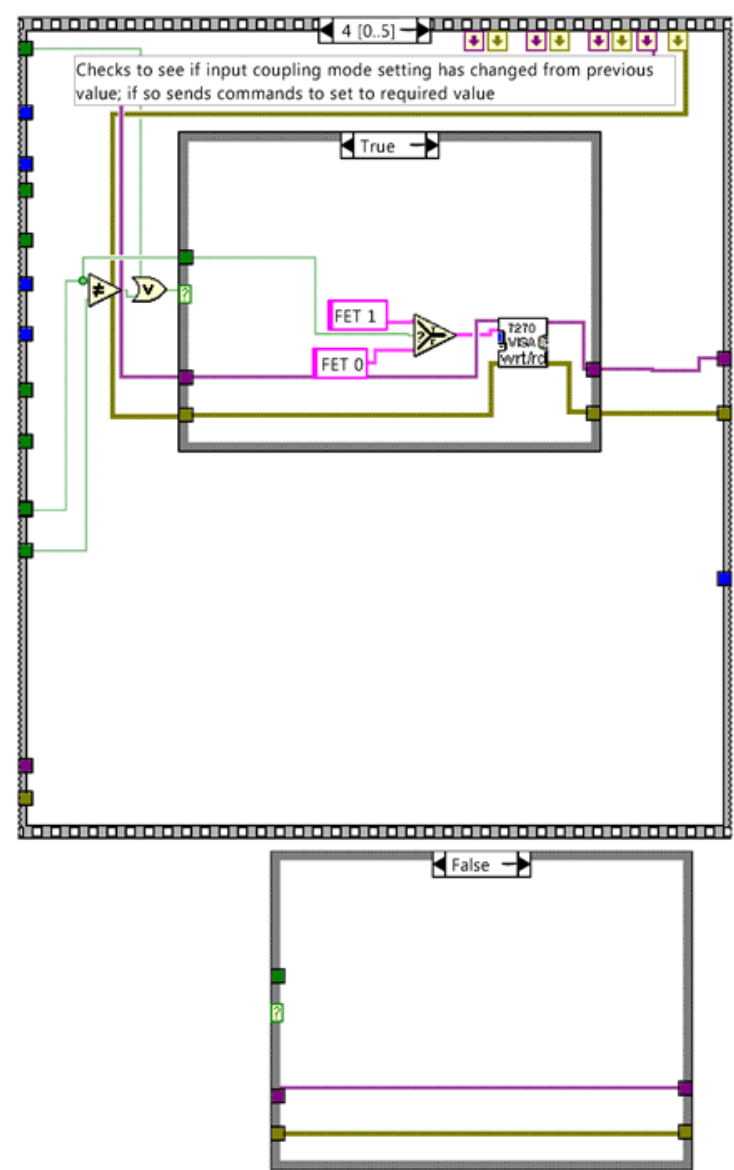
SR7270 Configure Signal Channel Input.vi

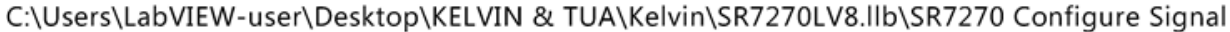

Channel Input.vi

Last modified on 5/11/2009 at 6:57 PM

Printed on 5/20/2016 at 8:04 PM

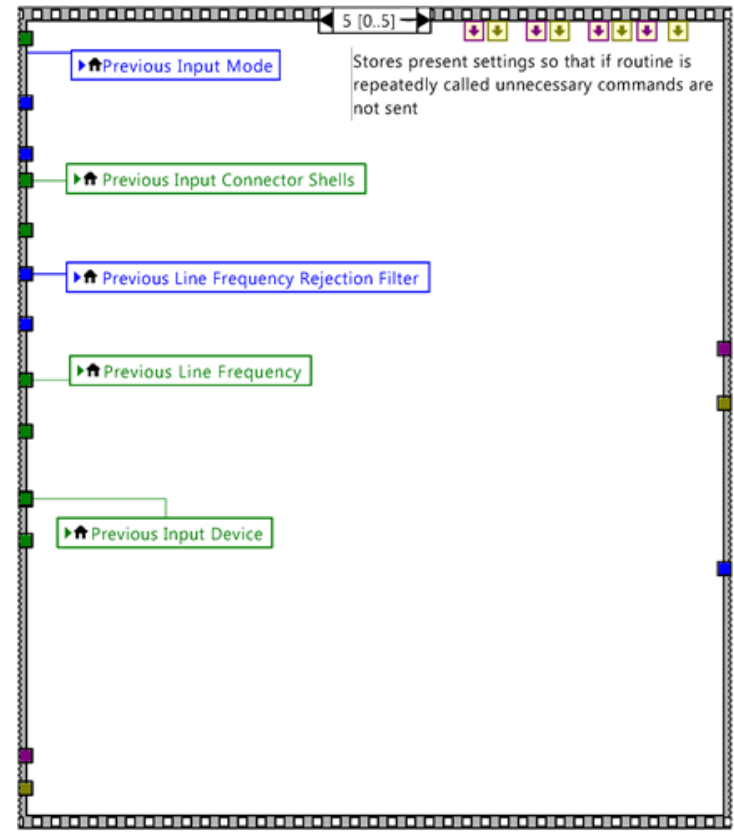


Appendix 34 Cryo3-SubVI: SR7270 Configure Virtual Reference Mode.vi 
SR7270 Configure Virtual Reference Mode.vi

C:\Users\LabVIEW-user\Desktop\KELVIN \& TUA\Kelvin\SR7270LV8.IIb\SR7270 Configure Virtual

Reference Mode.vi

Last modified on 5/11/2009 at 7:05 PM

Printed on 5/20/2016 at 6:39 PM

\section{Connector Pane}

\section{SR7270 Configure Virtual Reference Mode.vi}

$\begin{gathered}\text { VISA session (for class) } \\ \text { Virtual Reference Mode } \\ \text { error in (no error) }\end{gathered}-$ VISA session

This VI turns the Virtual Reference Mode on or off.

12 March 2009: First Release

\section{Front Panel}

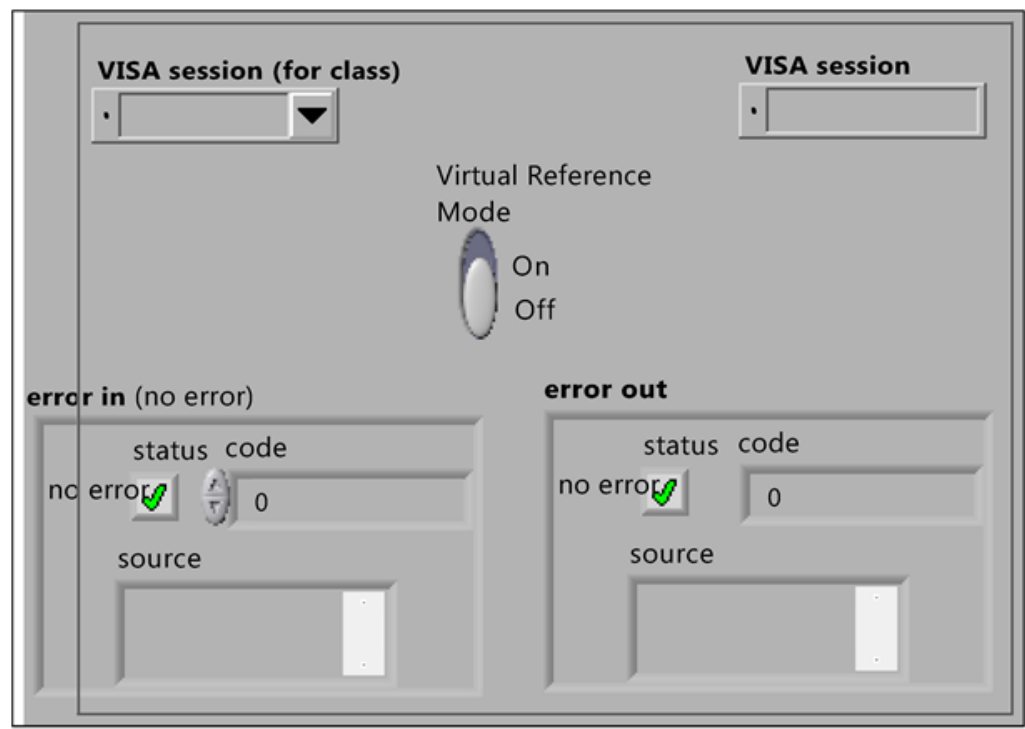


SR7270 Configure Virtual Reference Mode.vi

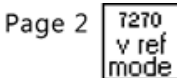

C:\Users\LabVIEW-user\Desktop\KELVIN \& TUA\Kelvin\SR7270LV8.IIb\SR7270 Configure Virtual

Reference Mode.vi

Last modified on 5/11/2009 at 7:05 PM

Printed on 5/20/2016 at 6:39 PM

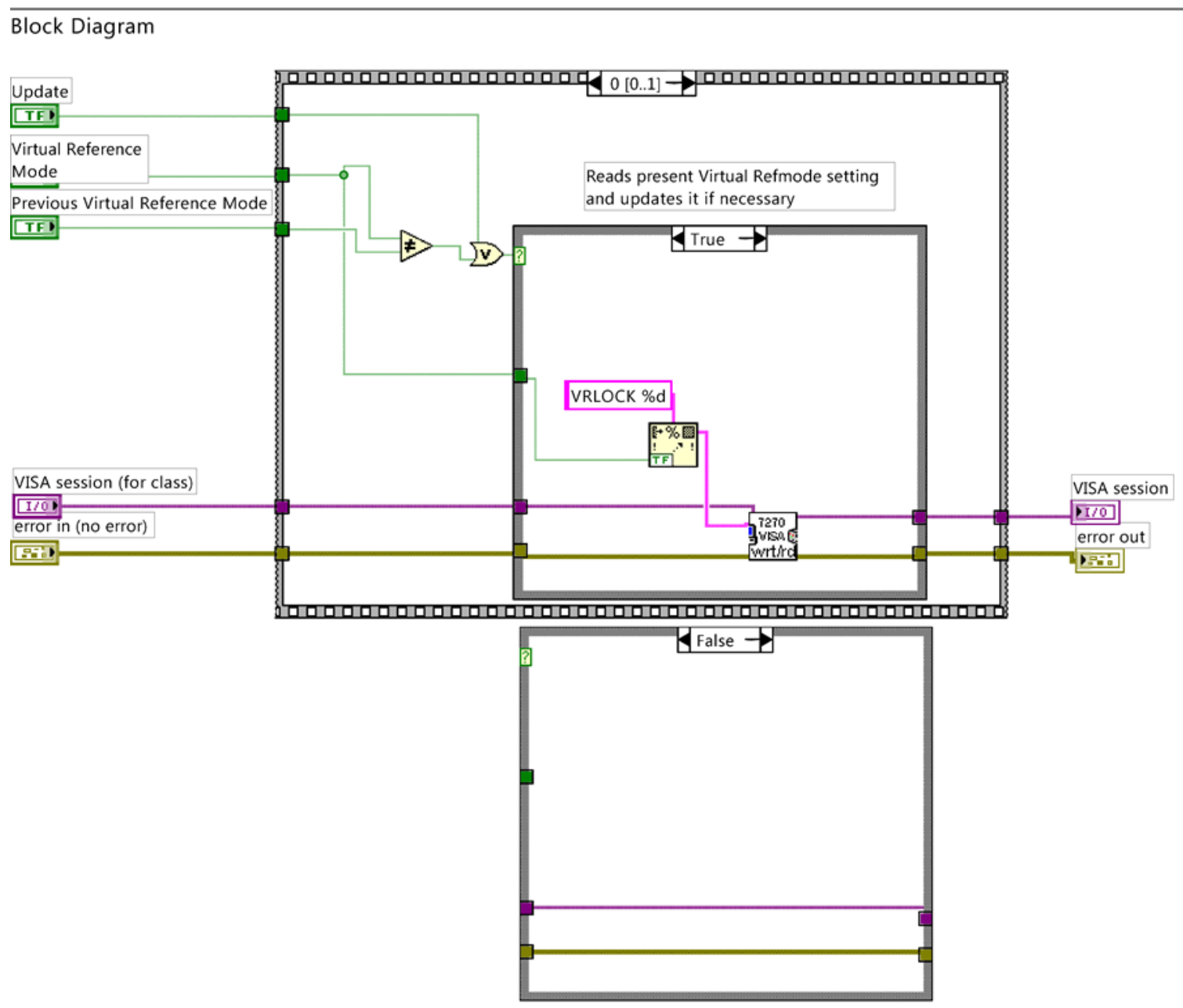


SR7270 Configure Virtual Reference Mode.vi

C:\Users\LabVIEW-user\Desktop\KELVIN \& TUA\Kelvin\SR7270LV8.IIb\SR7270 Configure Virtual Reference Mode.vi

Last modified on 5/11/2009 at 7:05 PM

Printed on 5/20/2016 at 6:39 PM

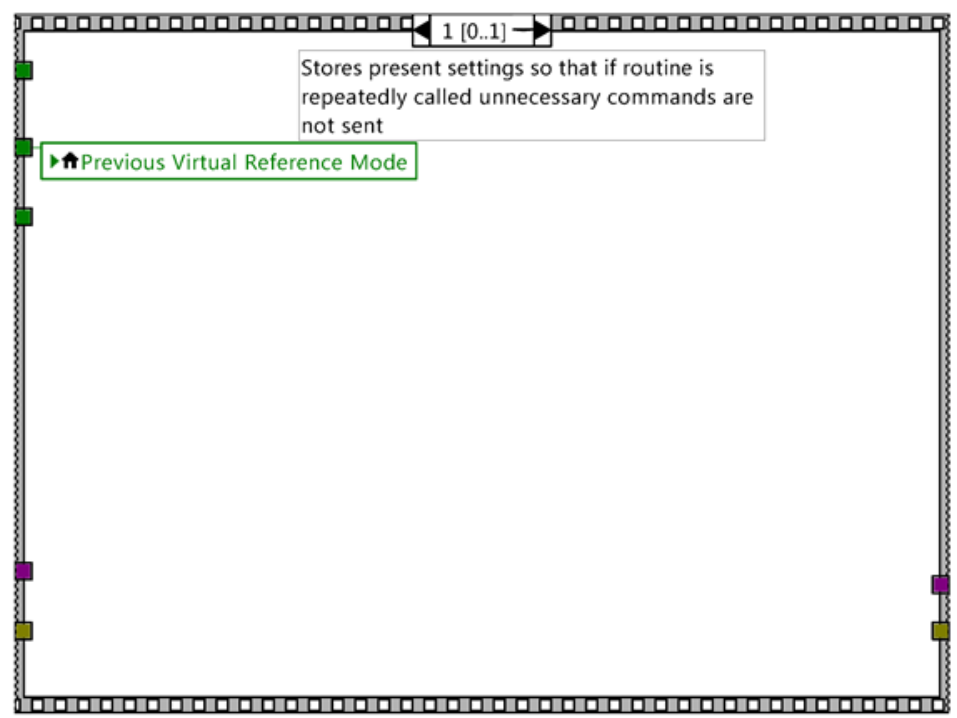


Appendix 35 Cryo3-SubVI: SR7270 Initialization.vi 
SR7270 Initialize.vi

C:\Users\LabVIEW-user\Desktop\KELVIN \& TUA\Kelvin\SR7270LV8.IIb\SR7270 Initialize.vi

Last modified on 5/11/2009 at 6:57 PM

Printed on 5/20/2016 at 8:05 PM

Connector Pane

\section{SR7270 Initialize.vi}

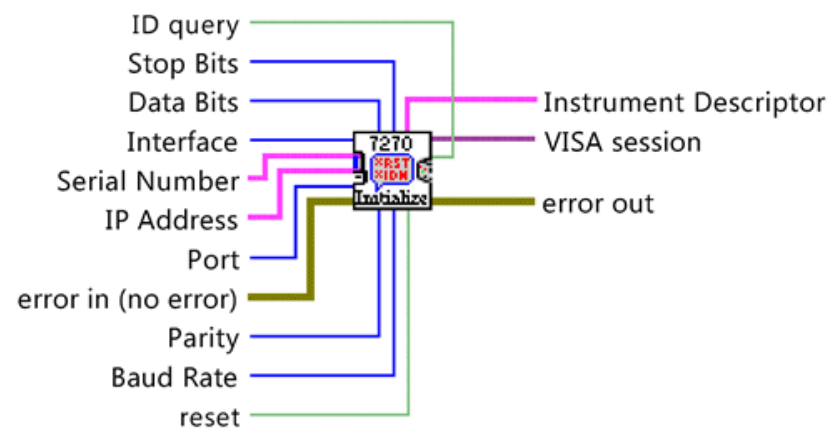

This VI passes the addressing information in the instrument decriptor to the Instr Open VI and returns the instrumeI ID. You can optionally reset the instrument by setting the front panel reset control. You must run this VI before usi any of the instrument driver VIs for this instrument.

\section{May 2009: First Release}

Front Panel

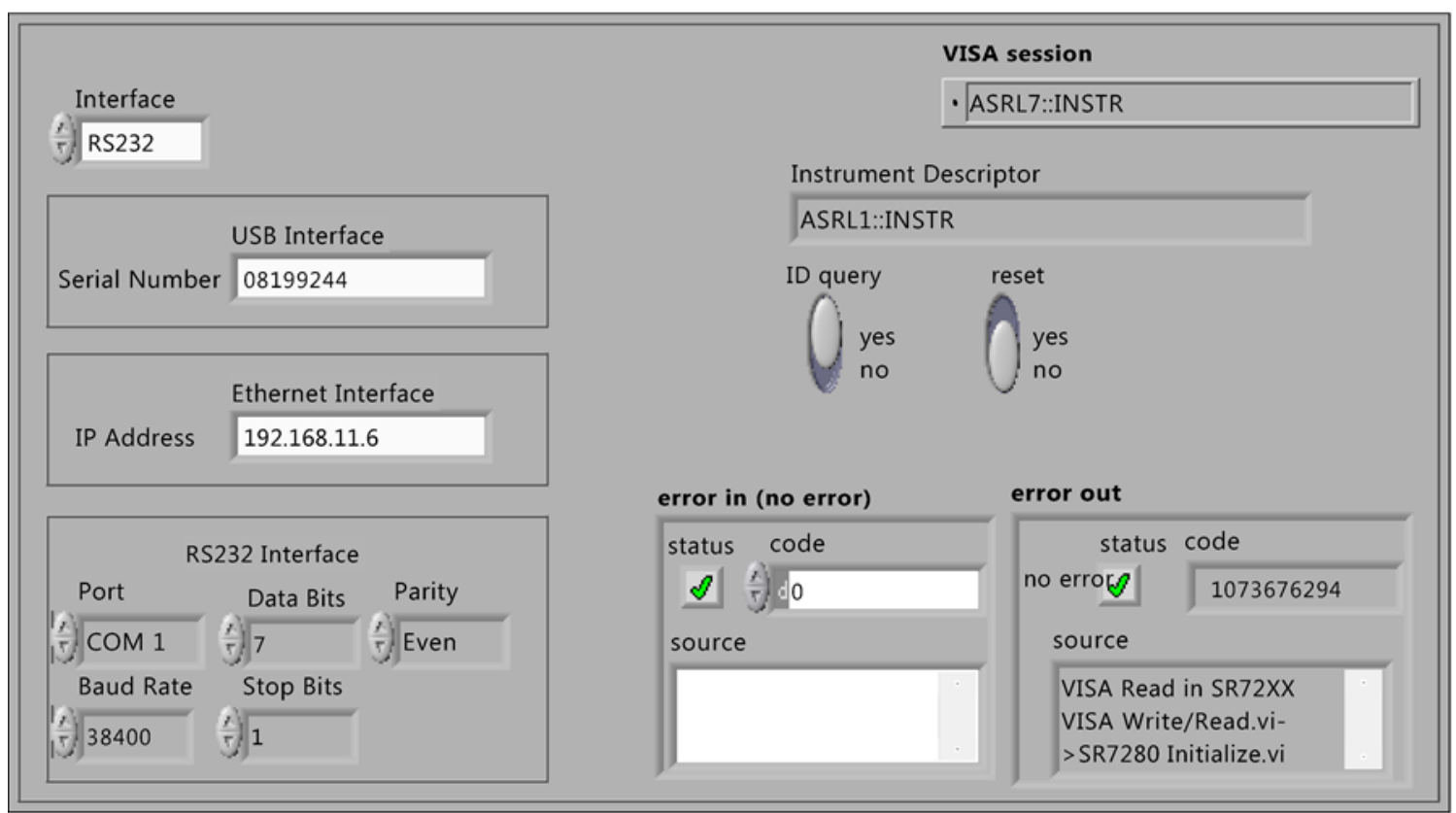


SR7270 Initialize.vi

C:\Users\LabVIEW-user\Desktop\KELVIN \& TUA\Kelvin\SR7270LV8.IIb\SR7270 Initialize.vi

Page 2 3270 Last modified on 5/11/2009 at 6:57 PM

Printed on 5/20/2016 at 8:05 PM

Block Diagram

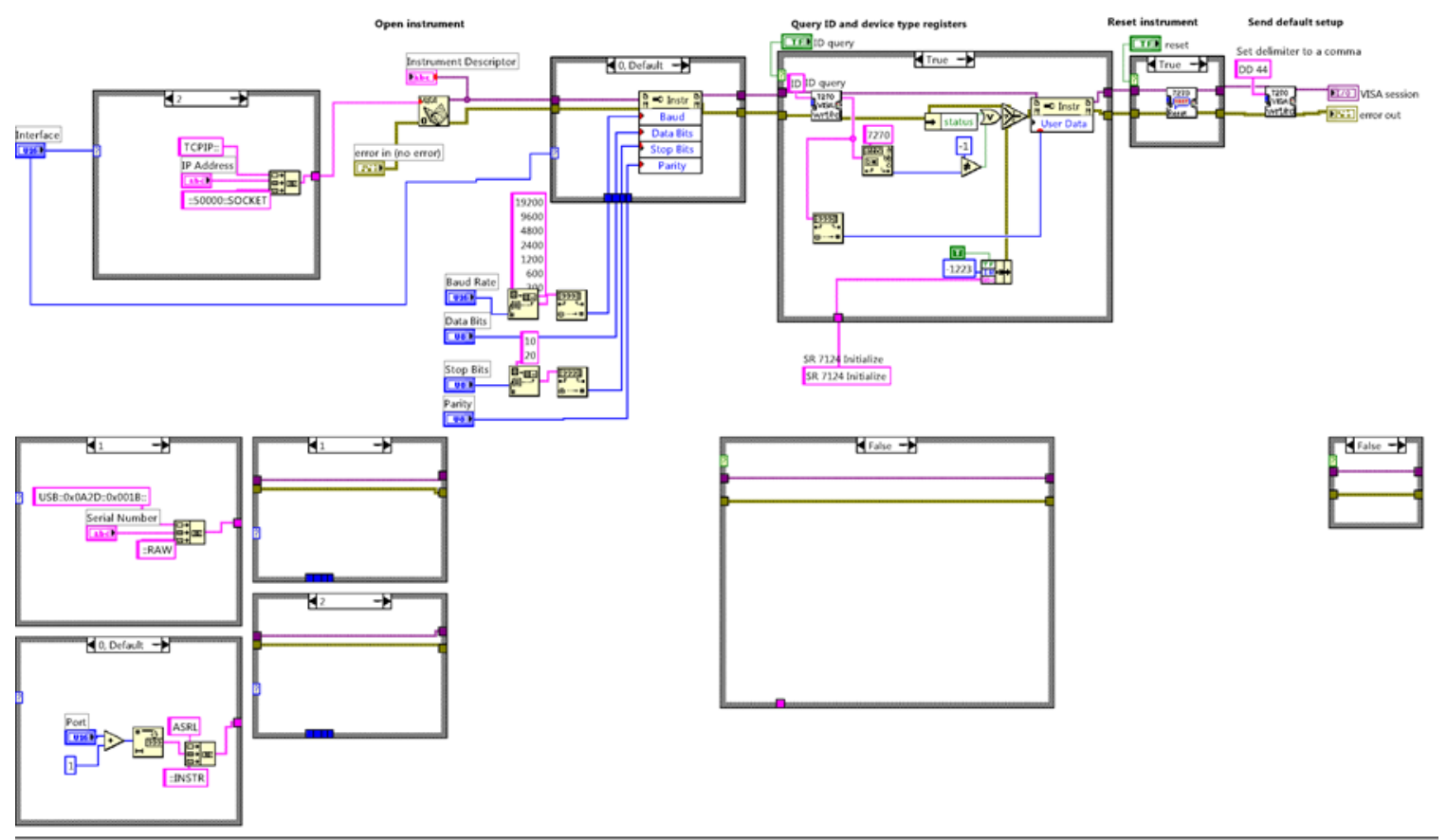


Appendix 36 Cryo3- SubVI: SR7270 VISA WriteRead.vi 
SR7270 VISA Write/Read.vi

C:\Users\LabVIEW-user\Desktop\KELVIN \& TUA\Kelvin\SR7270LV8.IIb\SR7270 VISA Write/Read.vi

Last modified on 5/11/2009 at 7:02 PM

Printed on 5/20/2016 at 8:00 PM

Connector Pane

\section{SR7270 VISA Write/Read.vi}

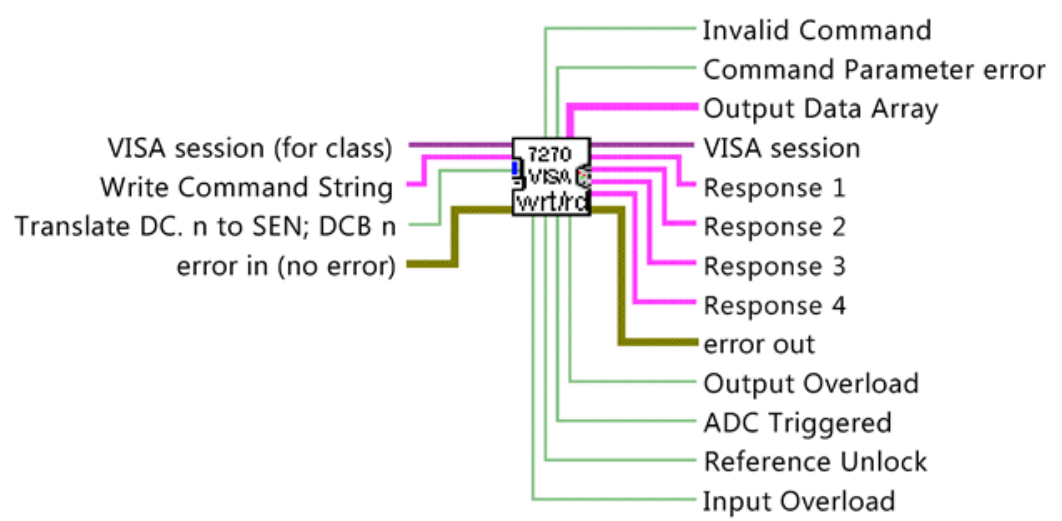

This VI should be used instead of standard VISA write or write-read constructs since it supports the software handshaking required when using the RS232 interface. It also handles correctly the USB and Ethernet interfaces, anc the following types of instrument response:-

Single response, e.g. to $X$ (output) command Multiple responses, e.g. to $\mathrm{M}$ (monitor) command

Array responses, e.g. to DC (dump curve) command

Response to * (speed transfer) command

It also reports the instrument's status

12 May 2009: First Release 
SR7270 VISA Write/Read.vi

C:IUsers\LabVIEW-user\Desktop\KELVIN \& TUA\Kelvin\SR7270LV8.IIb\SR7270 VISA Write/Read.vi Last modified on 5/11/2009 at 7:02 PM

Printed on 5/20/2016 at 8:00 PM

Front Panel

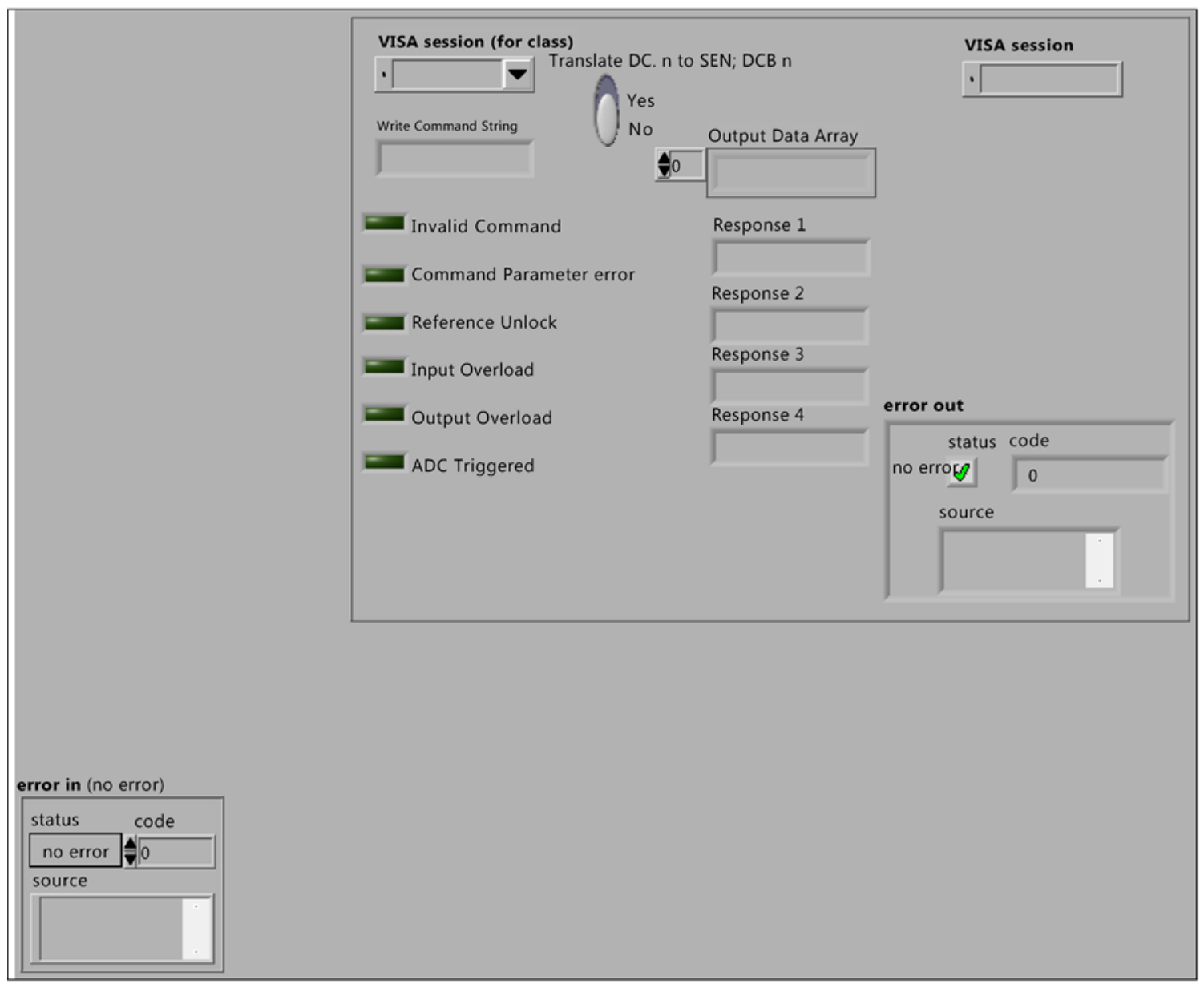


SR7270 VISA Write/Read.vi

C:\Users\LabVIEW-user\Desktop\KELVIN \& TUA\Kelvin\SR7270LV8.IIb\SR7270 VISA Write/Read.vi Last modified on 5/11/2009 at 7:02 PM

Printed on 5/20/2016 at 8:00 PM

Block Diagram

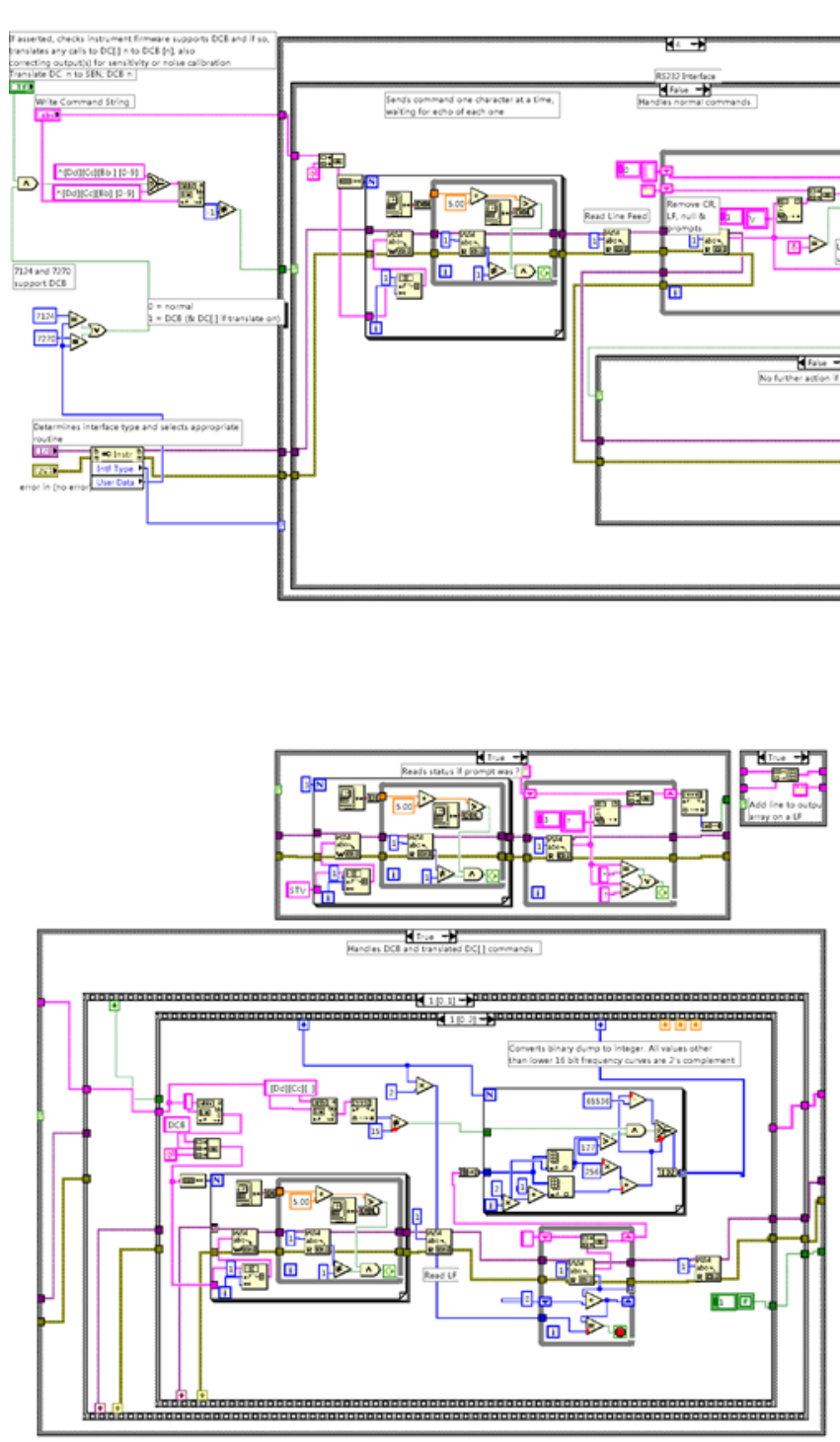


SR7270 VISA Write/Read.vi

C:\Users\LabVIEW-user\Desktop\KELVIN \& TUA\Kelvin\SR7270LV8.IIb\SR7270 VISA Write/Read.vi Last modified on 5/11/2009 at 7:02 PM

Printed on 5/20/2016 at 8:00 PM

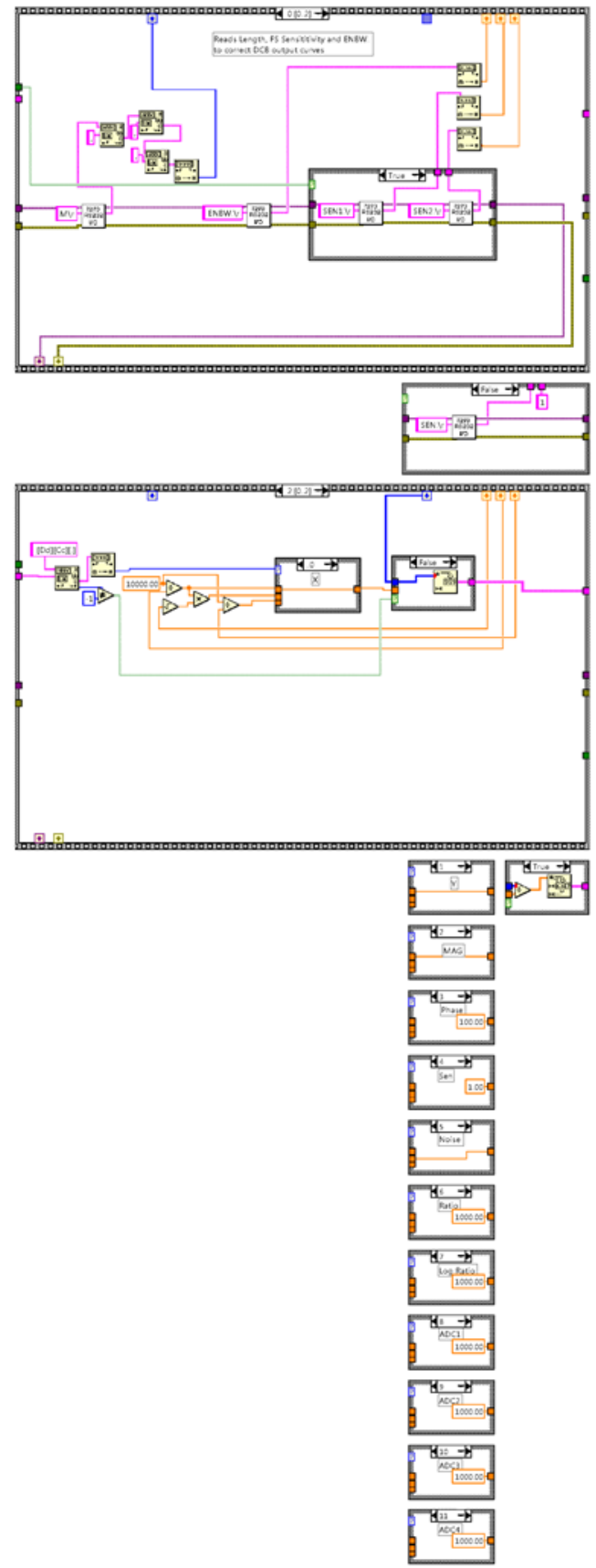


C:IUsers\LabVIEW-user\Desktop\KELVIN \& TUA\Kelvin\SR7270LV8.IIb\SR7270 VISA Write/Read.vi Last modified on 5/11/2009 at 7:02 PM

Printed on 5/20/2016 at 8:00 PM

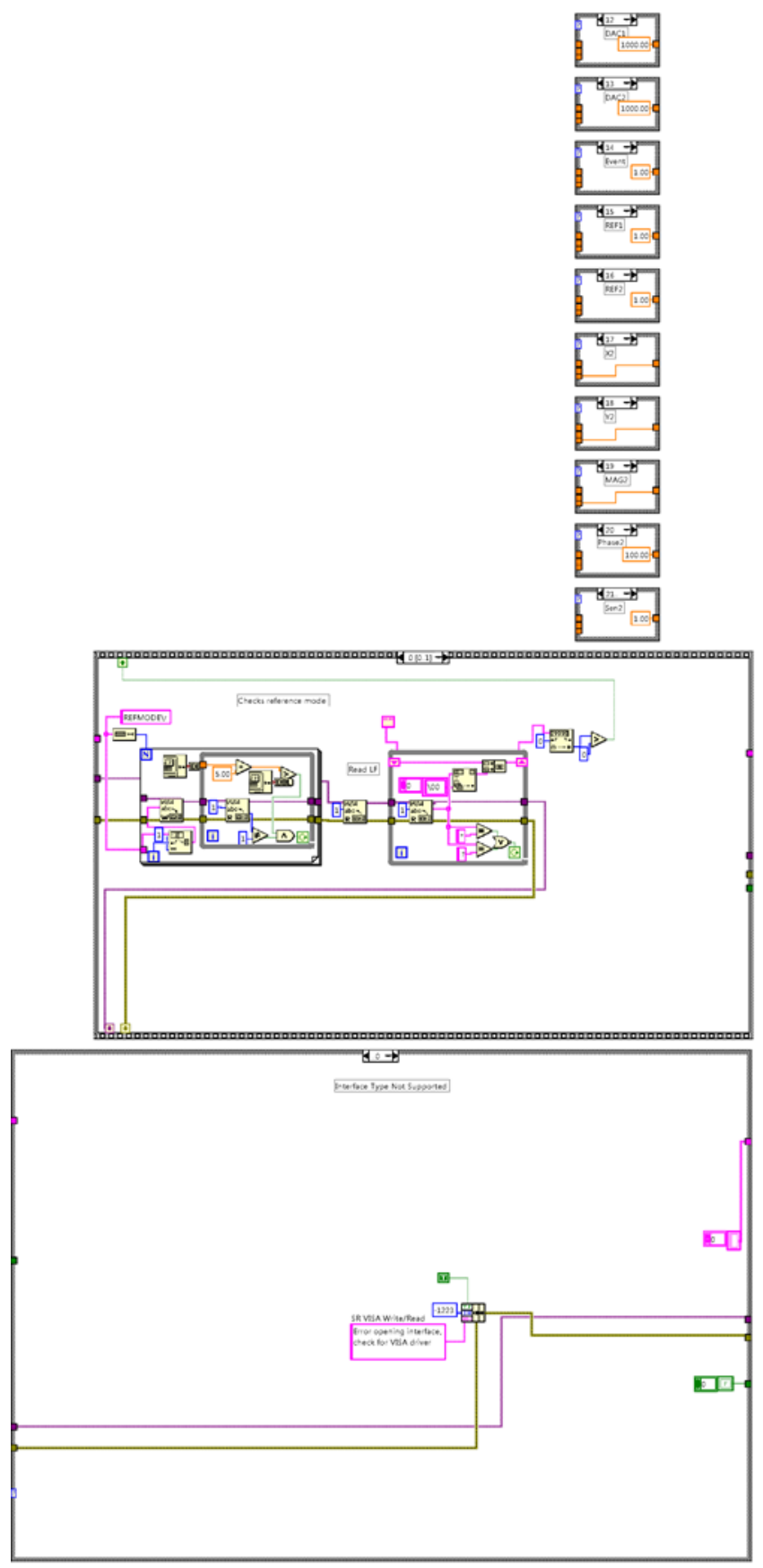


C:IUsers\LabVIEW-user\Desktop\KELVIN \& TUA\Kelvin\SR7270LV8.IIb\SR7270 VISA Write/Read.vi Last modified on 5/11/2009 at 7:02 PM

Printed on 5/20/2016 at 8:00 PM

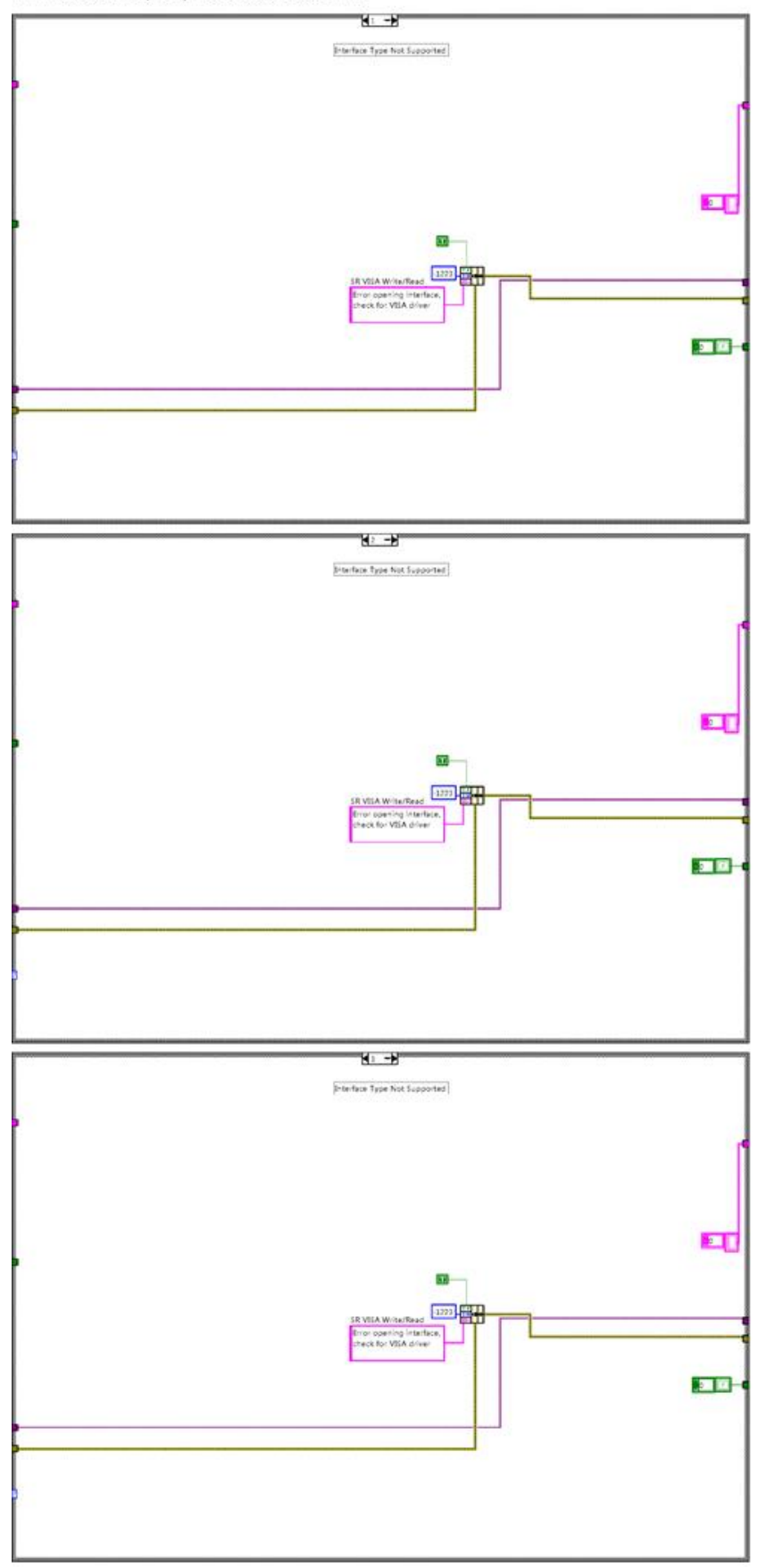


SR7270 VISA Write/Read.vi

C:IUsers\LabVIEW-user\Desktop\KELVIN \& TUA\Kelvin\SR7270LV8.IIb\SR7270 VISA Write/Read.vi Last modified on 5/11/2009 at 7:02 PM

Printed on 5/20/2016 at 8:00 PM

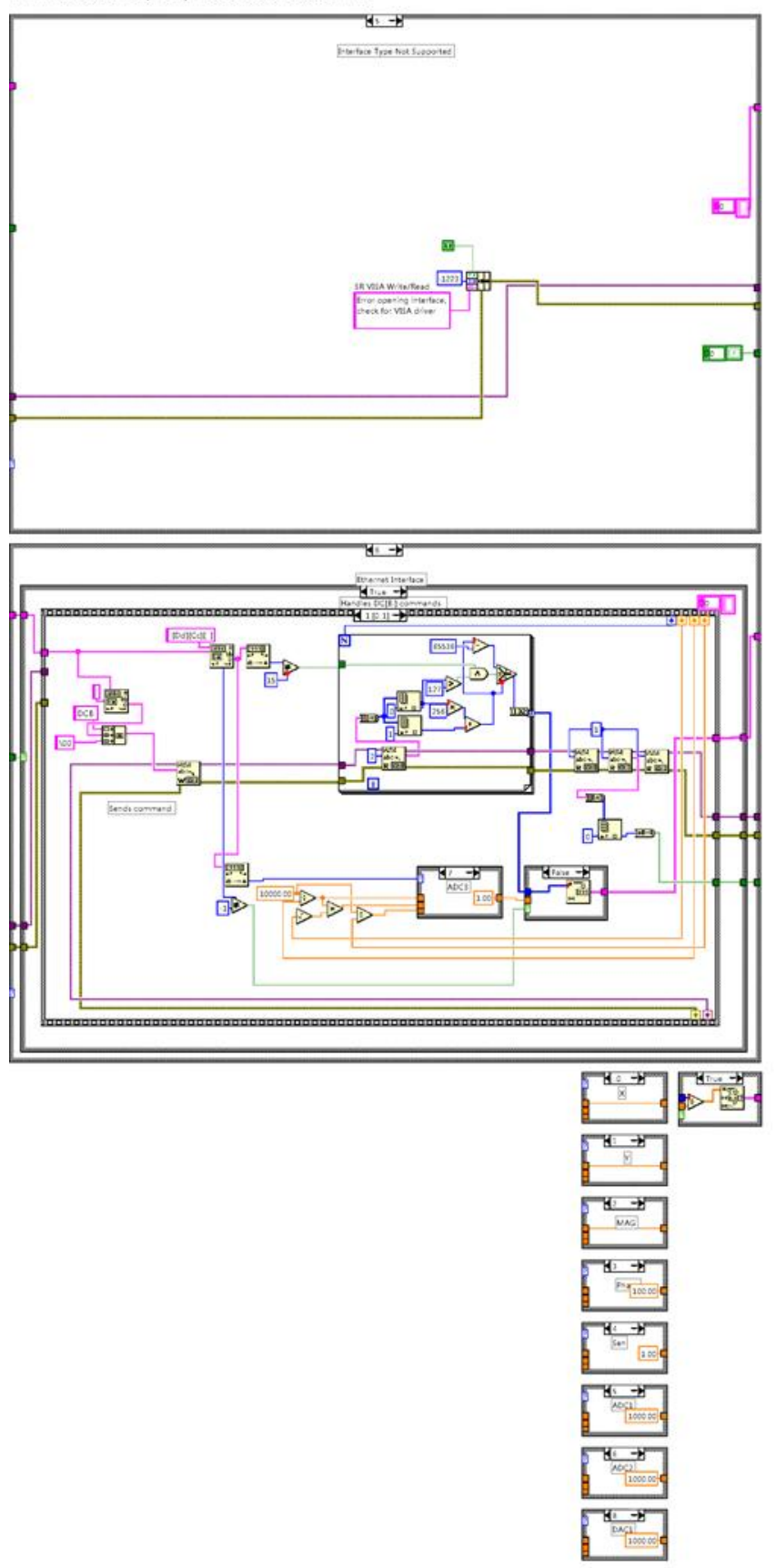


SR7270 VISA Write/Read.vi

C:\Users\LabVIEW-user\Desktop\KELVIN \& TUA\Kelvin\SR7270LV8.IIb\SR7270 VISA Write/Read.vi Last modified on 5/11/2009 at 7:02 PM

Printed on 5/20/2016 at 8:00 PM

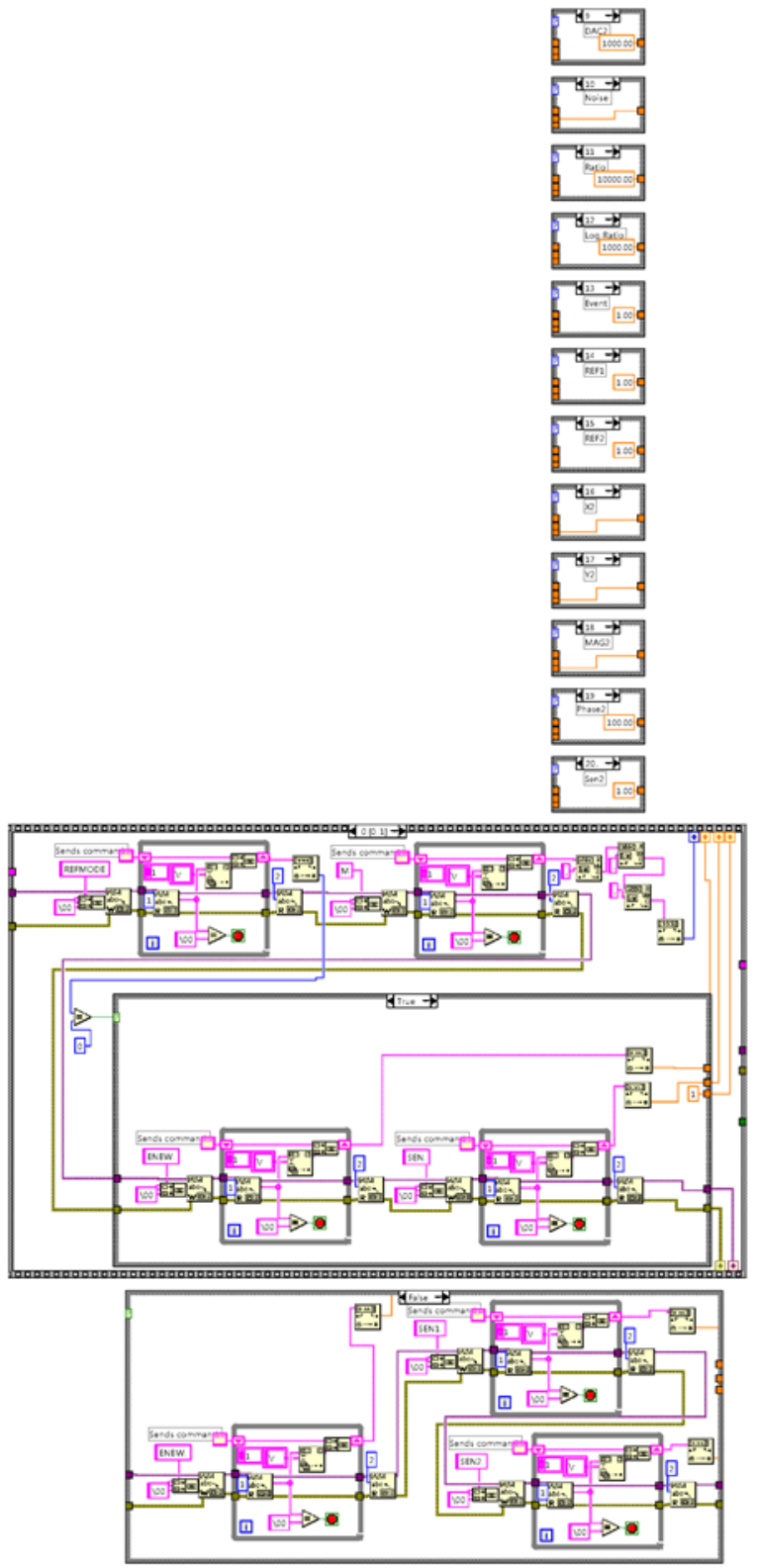


SR7270 VISA Write/Read.vi

C:\Users\LabVIEW-user\Desktop\KELVIN \& TUA\Kelvin\SR7270LV8.IIb\SR7270 VISA Write/Read.vi Last modified on 5/11/2009 at 7:02 PM

Printed on 5/20/2016 at 8:00 PM
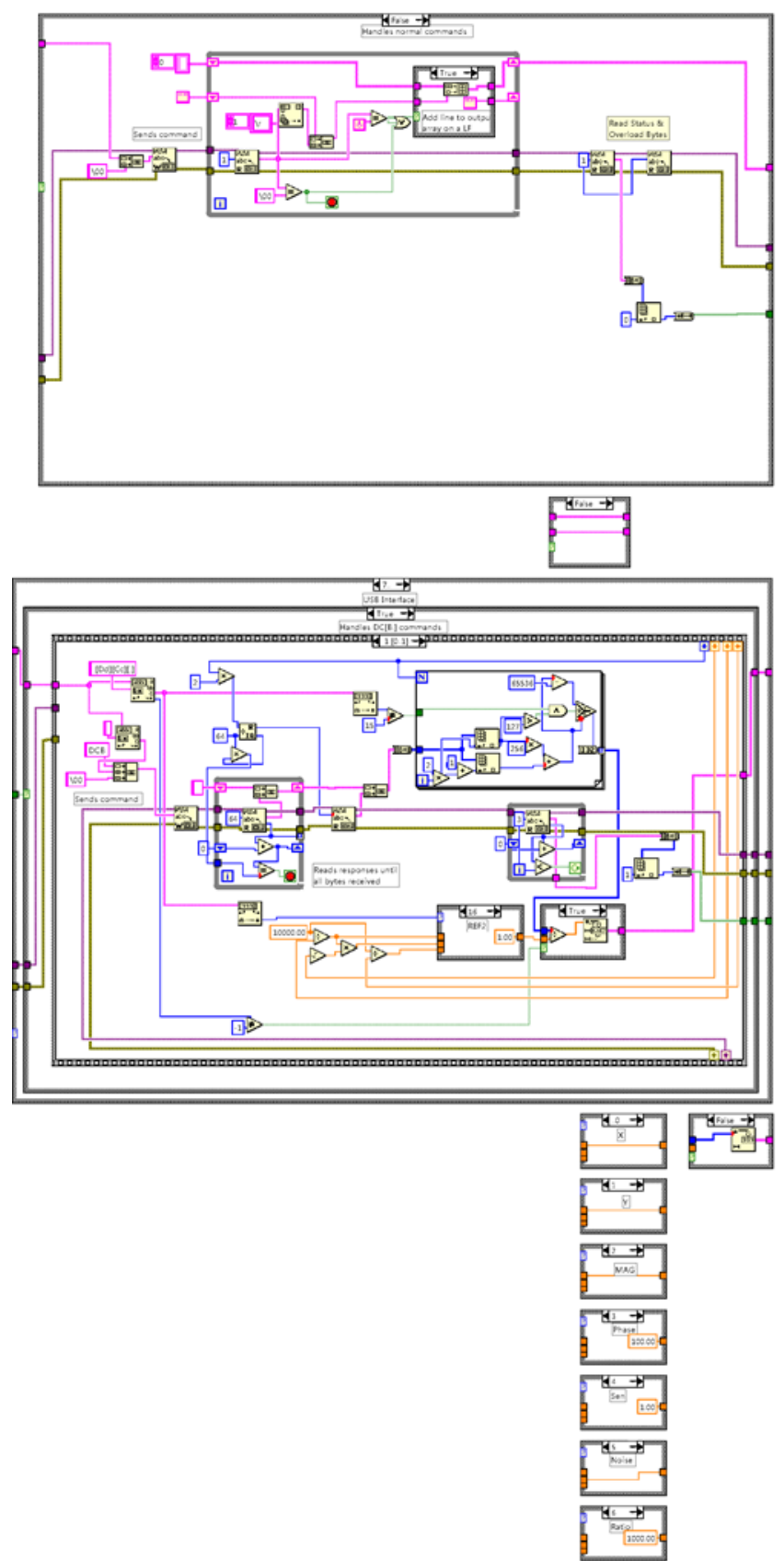
SR7270 VISA Write/Read.vi

C:IUsers\LabVIEW-user\Desktop\KELVIN \& TUA\Kelvin\SR7270LV8.IIb\SR7270 VISA Write/Read.vi Last modified on 5/11/2009 at 7:02 PM

Printed on 5/20/2016 at 8:00 PM

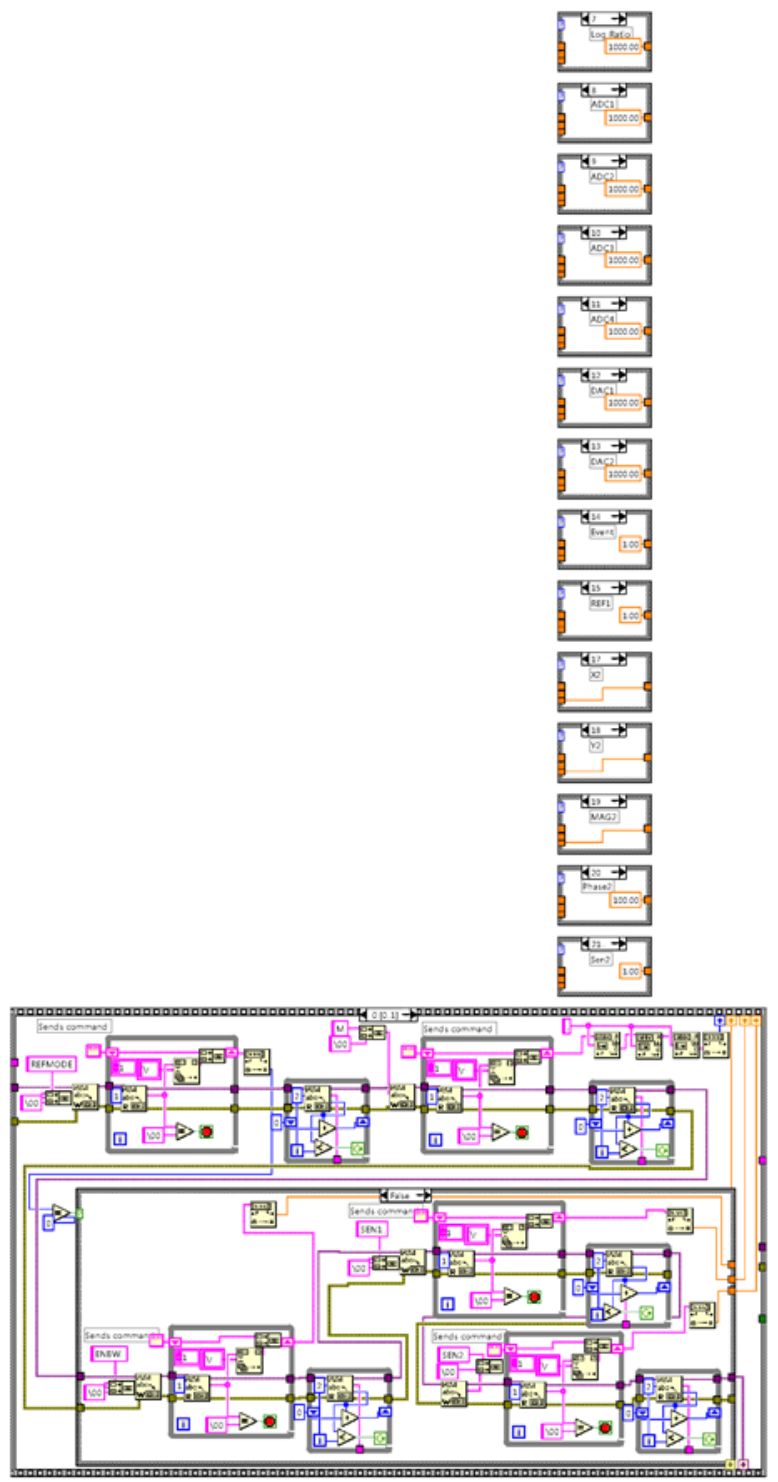


SR7270 VISA Write/Read.vi

C:\Users\LabVIEW-user\Desktop\KELVIN \& TUA\Kelvin\SR7270LV8.IIb\SR7270 VISA Write/Read.vi Last modified on 5/11/2009 at 7:02 PM

Printed on 5/20/2016 at 8:00 PM
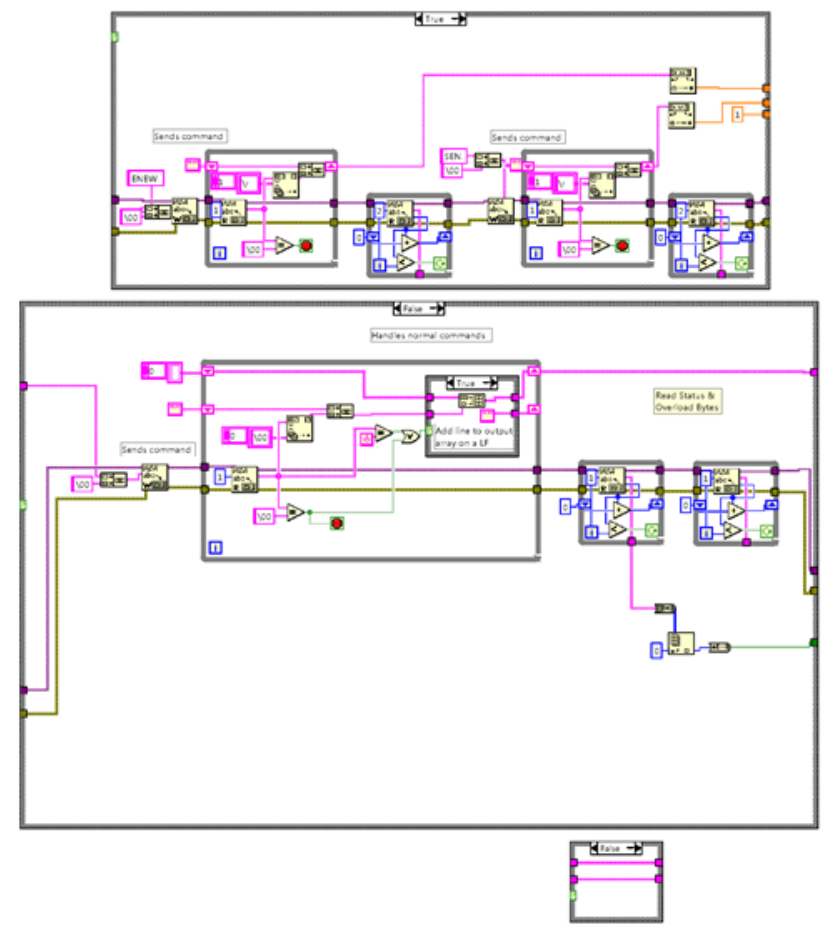
Appendix 37 Cryo4-Delta Measure ver 23 Auto compressor and two

separate files generated- VI name was disconnected on cooling in previous

version.vi 
Cryo4-Delta Measure ver 23 Auto compressor and two separate files generated-VI name was disconnect C:\Users\LabVIEW-user\AppData\Local\Temp\Cryo4-Delta Measure ver 23 Auto compressor and two separate files generated- VI name was disconnected on cooling in previous version.vi Last modified on 5/20/2016 at 5:21 PM

Printed on 5/20/2016 at 5:36 PM

Cryo4-Delta Measure ver 23 Auto compressor and two separate files generated- VI name was disconnected
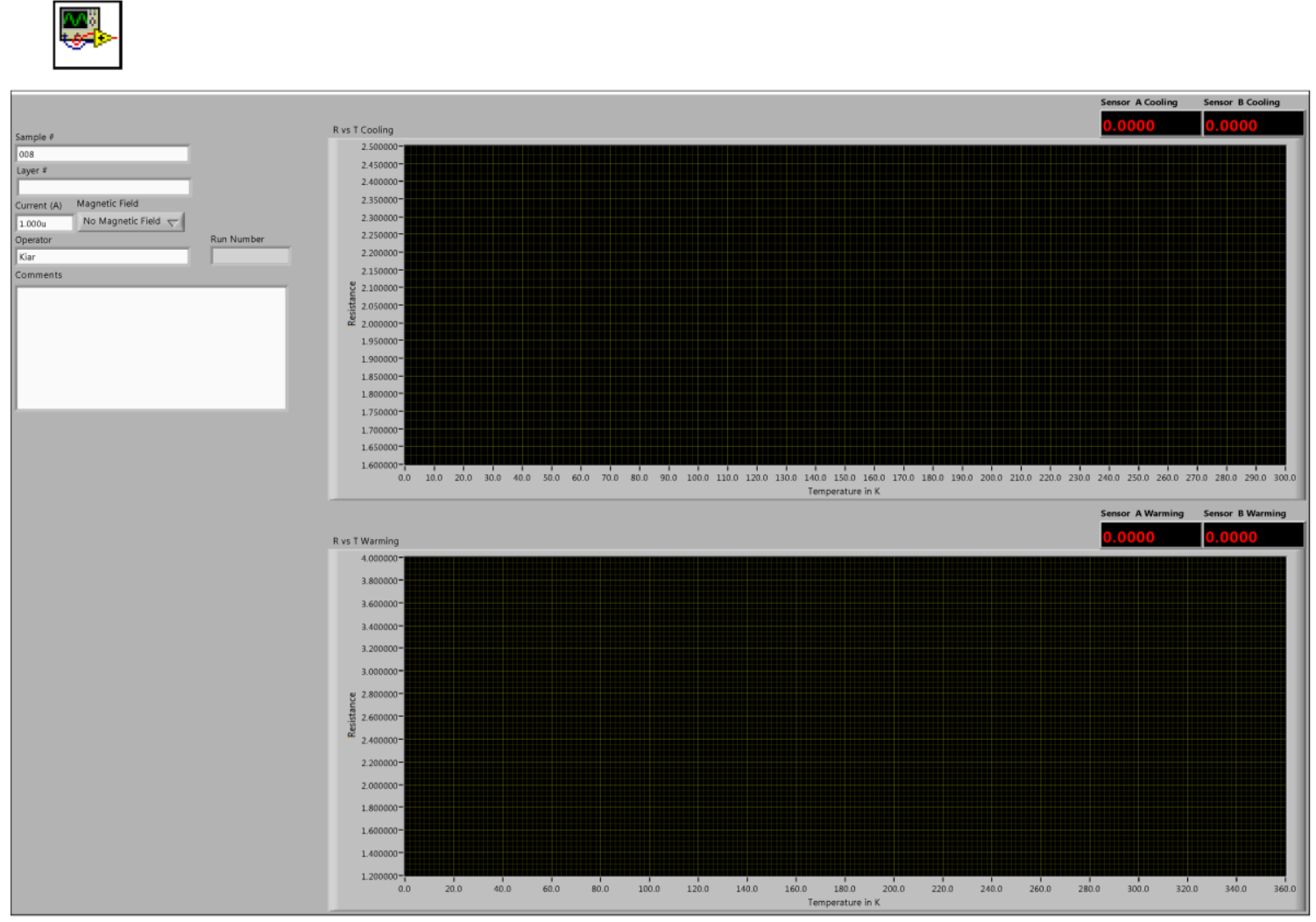
Cryo4-Delta Measure ver 23 Auto compressor and two separate files generated- VI name was disconnect

$C: \backslash$ Users $\backslash$ LabVIEW-user $\backslash A p p D a t a \backslash L o c a l \backslash T e m p \backslash C r y o 4-D e l t a$ Measure ver 23 Auto compressor and two separate files generated-VI name was disconnected on cooling in previous version.vi

Last modified on 5/20/2016 at 5:21 PM

Printed on 5/20/2016 at 5:36 PM

on cooling in previous version.vi 
Cryo4-Delta Measure ver 23 Auto compressor and two separate files generated-VI name was disconnect $C: \backslash$ Users $\backslash$ LabVIEW-user $\backslash A p p D a t a \backslash L o c a l \backslash T e m p \backslash C r y o 4-D e l t a$ Measure ver 23 Auto compressor and two separate files generated- VI name was disconnected on cooling in previous version.vi Last modified on 5/20/2016 at 5:21 PM

Printed on 5/20/2016 at 5:36 PM
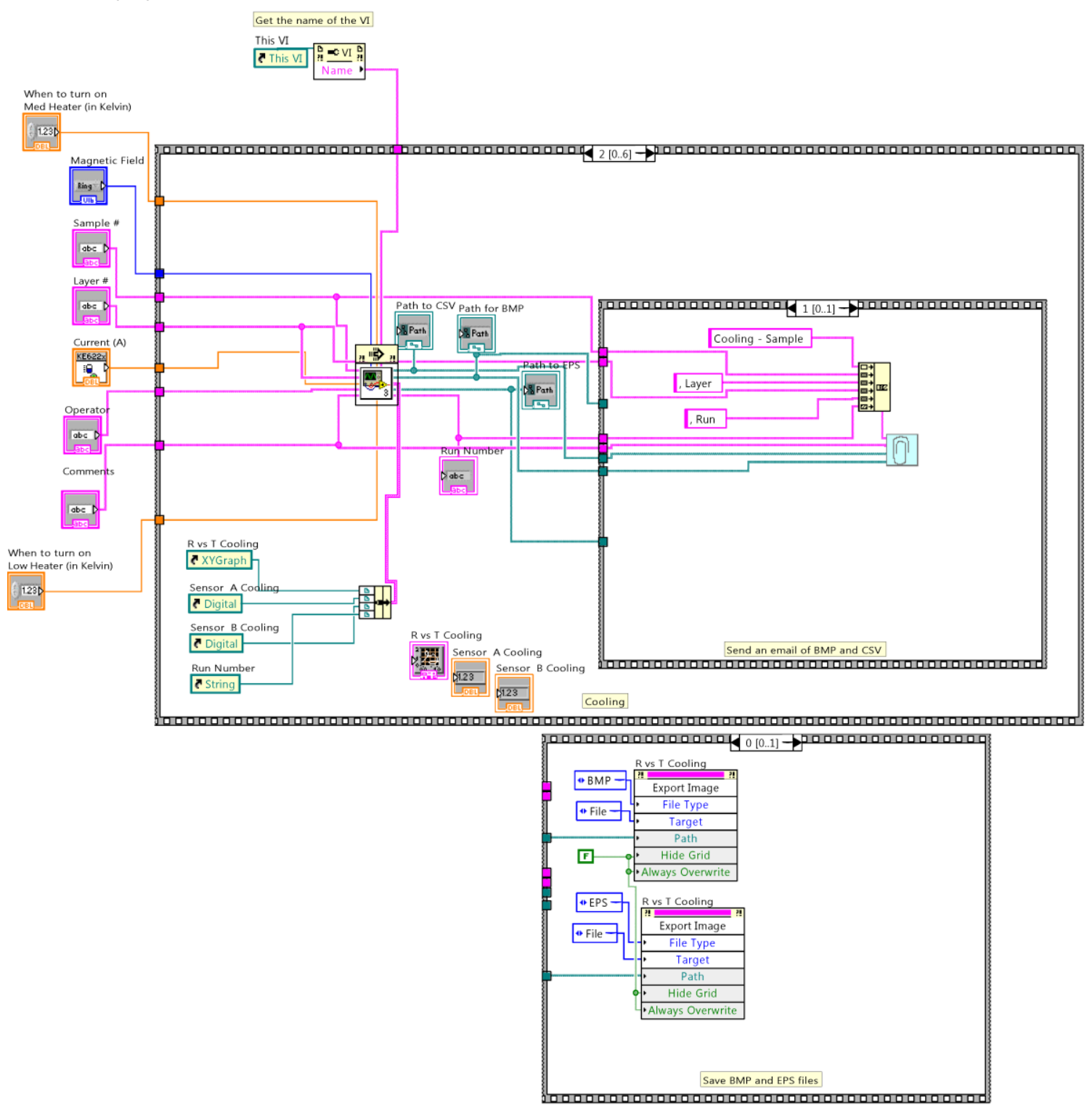
Appendix $38 \quad$ F70 read all and command_SubVI.vi 
F70 read all and command_SubVI.vi

C: $\backslash$ Users\LabVIEW-user\AppData\Local\Temp\F70 read all and command_SubVI.vi

Last modified on 5/20/2016 at 5:58 PM

Printed on 5/20/2016 at 5:58 PM

Connector Pane

F70 read all and command_SubVI.vi

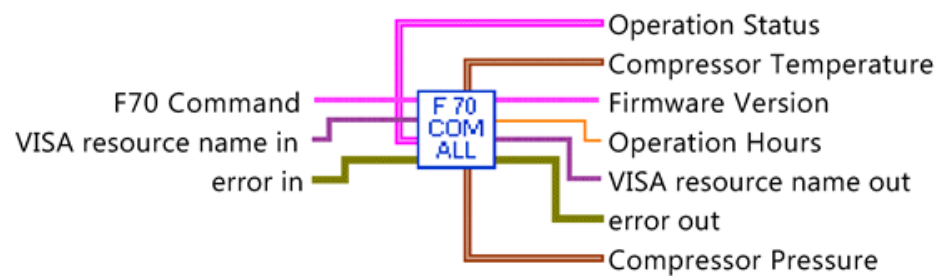

F-70 compressor RS232 communication driver.

This VI can directly output reading of $\mathrm{T} 1, \mathrm{~T} 2, \mathrm{~T} 3, \mathrm{P} 1, \mathrm{P} 2$, Firmware version, operation hours, and compressor statu: (state of operation, alarms etc.). It can also send operation command (turn ON/OFF compressor and cold head, et when compressor is in configuration mode 1.

It can be used as a sub-vi in other program.

It takes two inputs:

1. VISA resource name (COM1, COM2, etc.)

2. F70 command (you can choose from the drop down list (Turn on compressor and cold head, turn of compressc and cold head, cold head pause, read all temperatures, read all pressures, read status bits etc.)

It outputs the following (depending on the input F70 command):

1. Compressor temperature $(\mathrm{T} 1, \mathrm{~T} 2, \mathrm{~T} 3)$

2. Compressor Pressure (P1, P2)

3. Compressor status bits (configuration mode, state of operation, alarms, system on, etc.)

4. Firmware version and operation hours.

General Information about F-70 RS232 communication protocol.

This section describes the F-70 compressor RS232 interface only. It does not describe overall operation or safety of the F-70 compressor. Please refer to the F-70 operating manual for operating instructions and warnings. The information in this section should be used only after safe operation of the F-70 compressor is understood.

1. F-70 Front Panel Connection: Male DB-9 connector

Pin 2 = Receive

Pin $3=$ Transmit

Pin 5 = Earth Ground (connected to compressor chassis ground)

2. RS232 parameters.

a. Baud - 9600

b. No parity

c. 8 bit data

d. 1 bit stop 
F70 read all and command_SubVI.vi

C:IUsers \LabVIEW-user\AppData\Local\Temp\F70 read all and command_SubVI.vi

Last modified on 5/20/2016 at 5:58 PM

Printed on 5/20/2016 at 5:58 PM

The F-70 sends no unsolicited messages. It only responds to commands from a host computer.

\section{NOTE}

RS232 commands that change operation of the F-70 compressor (on, off, reset, cold head run, cold head pause a pause off) have equal value as front panel key press control. The F-70 will respond equally to either source of contr input in the order in which it is received.

\section{NOTE}

There are no error routines or checks to prevent possible conflicts if a compressor is both connected to a diagnostic interface (DB25) control element and an RS232 control element except:

1. RS232 commands that change operation of the F-70 compressor are active only when the compressor is set for DB25 Configuration Mode 1 (reference operating manual). RS232 commands that transmit data are active for bo DB25 Configuration Mode 1 and Mode 2.

2. In Configuration Mode 1, active DB25 operating signals will take priority over front panel key press and RS232 control. DB25 Configuration Mode 2 does not permit either RS232 or front panel key press control.

RS232 Command List (See the Command Set section below for command descriptions and examples)

1. Information Commands

\$TEA: Read all temperatures \$TEn: Read temperature $n(n=1,2,3$, or 4$)$

\$PRA: Read all pressures \$PRn: Read pressure $n(n=1$ or 2$)$

\$STA: Read status bits \$ID1: Read firmware version and elapsed operating hours

2. Operating Commands

\$ON1: On \$OFF: Off

\$RS1: Reset \$CHR: Cold head run

\$CHP: Cold head pause \$POF: Cold head pause off

3. Responses: \$???: Invalid command received

Command Structure

The individual bytes of any communication packet (frame) are restricted to the ASCII domain of $0 \times 20(20 \mathrm{H}$, space through $0 \times 7 E(7 E H$, tilde), plus 0x0D (carriage return).

The basic communication frame from the host computer to the F-70 compressor is comprised of a start character command mnemonic, data (if required), checksum, and a message end character.

Start Character: This is always $0 \times 24(24 \mathrm{H}$, dollar sign $)$.

Command: This is a 3 character mnemonic. It defines the action which will be taken by the controller.

Data: Data will be transmitted as ASCII text equivalents. Example: the number 123 will be text "123". In the event t

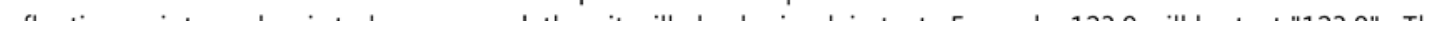


F70 read all and command_SubVI.vi

C:\Users\LabVIEW-user\AppData\Local\Temp\F70 read all and command_SubVI.vi

Last modified on 5/20/2016 at 5:58 PM

Printed on 5/20/2016 at 5:58 PM

a tIoatıng point number is to be conveyea, then It wIII also be in plain text. Exampie: $1 \angle 3 . y$ wIII be text " $1 \angle 3 . y$ ". Ine data field length is fixed depending on the command that is issued. This will be defined at the command level.

Checksum (CRC-16): The checksum is a 16 bit CRC (CRC-16). It is transmitted as four-digit ASCII hex. Example: a bit binary checksum "001001110101011" in four-digit ASCII equals "23AB". The check sum calculation includes the "\$" start character, command field, and data field (if present). See section E below for description of CRC-16 generation.

End of message: This is always $0 \times 0 \mathrm{D}(0 \mathrm{DH}$ carriage return). This assures that the end of message character falls outs the standard text domain and will not be incorporated in commands, data, or checksums.

The response frame from the F-70 compressor is similar to the command frame, but includes delimiters between various fields.

Start Character: This is always $0 \times 24(24 \mathrm{H}$, dollar sign $)$.

Command: This is a 3 character mnemonic. It is the same as (echoes) the command sent from the host controller

Delimiter: This is always $0 \times 2 \mathrm{C}(2 \mathrm{CH}$, comma).

Data: Data will be transmitted as ASCII text equivalents. Example: the number 123 will be text "123". In the event $t$ a floating point number is to be conveyed, then it will also be in plain text. Example: 123.9 will be text "123.9". The data field length is fixed depending on the command that is issued. This will be defined at the command level.

Checksum(CRC-16): The checksum is a 16 bit CRC. It is transmitted as four-digit ASCII hex. Example: a 16 bit bin checksum "001001110101011" in four-digit ASCII equals "23AB". The check sum calculation includes the "\$" start character, command field, data fields (if present), and all commas including the comma preceding the checksum. See section E below for description of CRC-16 generation.

End of message: This is always $0 \times 0 D(0 D H$ carriage return). This assures that the end of message character falls outs the standard text domain and will not be incorporated in commands, data, or checksums.

Command Set

\$TEA: Read all temperatures

Command with checksum and carriage return $=\$$ TEAA4B9 $<\mathrm{cr}\rangle$

Response: \$TEA,T1,T2,T3,T4,<crc-16><cr $>$

T1 through T4 are compressor internal temperatures in degrees $C$.

$\mathrm{T} 1$ = Compressor capsule helium discharge temperature

$\mathrm{T} 2=$ Water outlet temperature

$\mathrm{T} 3=$ Water inlet temperature

$\mathrm{T} 4$ is inactive (returns "000") for most F-70 variants. 
F70 read all and command_SubVI.vi

C:\Users\LabVIEW-user\AppData\Local\Temp\F70 read all and command_SubVI.vi

Last modified on 5/20/2016 at 5:58 PM

Printed on 5/20/2016 at 5:58 PM

$\mathrm{T} 1$ through $\mathrm{T} 4$ are compressor internal temperatures in degrees $\mathrm{C}$.

$\mathrm{T} 1$ = Compressor capsule helium discharge temperature

$\mathrm{T} 2$ = Water outlet temperature

$\mathrm{T} 3=$ Water inlet temperature

T4 is inactive (returns "000") for most F-70 variants.

The temperature fields are always 3 characters long and are rounded to the nearest degree. Temperatures less the $100{ }^{\circ} \mathrm{C}$ have leading zeroes.

Example: $\$$ TEA, $086,040,031,000,3798<\mathrm{cr}>$ corresponding to $\mathrm{T} 1=86^{\circ} \mathrm{C}, \mathrm{T} 2=40^{\circ} \mathrm{C}$,

$\mathrm{T} 3=31^{\circ} \mathrm{C}$ and where 3798 is the checksum and $\langle\mathrm{cr}\rangle$ is the carriage return.

\$Ten: Read selected temperature $(n=1,2,3$, or 4$)$

Command with checksum and carriage return $=\$$ TE140B8 <cr $>, \$ T E 241 F 8<$ cr $>, \$ T E 38139<$ cr $>$, or $\$ T E 44378<$ cr $>$

Response: \$TEn,Tn, $<$ crc-16 $><c r>$

Example: host sends $\$ T E 140 B 8 c r$. The response from the compressor is $\$ T E 1,086, A D B C<c r>$ corresponding to temperature $\mathrm{T} 1=86^{\circ} \mathrm{C}$ and where $\mathrm{ADBC}$ is the checksum and $\langle\mathrm{cr}\rangle$ is the carriage return.

\$PRA: Read all pressures

Command with checksum and carriage return $=\$$ PRA95F7 $\langle\mathrm{cr}\rangle$

Response: \$PRA, P1,P2, $<$ crc-16><cr $>$

P1 is the compressor return pressure in PSIG. P2 is inactive (returns "000") for most

F-70 variants.

The pressure fields are always 3 characters long and are rounded to the nearest whole number. Pressures less tha। 100 psig have leading zeroes.

Example: \$PRA,079,000,0CEC $<\mathrm{cr}>$ corresponding to $\mathrm{P} 1=79 \mathrm{psig}$ and where $0 \mathrm{CEC}$ is the checksum and $<\mathrm{cr}>$ is the carriage return.

\$PRn: Read selected pressure ( $\mathrm{n}=1$ or 2 )

Command with checksum and carriage return $=\$ P R 171 \mathrm{~F} 6<\mathrm{cr}>$ or $\$ \mathrm{PR} 270 \mathrm{~B} 6<\mathrm{cr}>$

Response: \$PRn, Pn, $<$ crc-16 $>\langle$ cr $>$

Example: host sends $\$ P R 171 \mathrm{~F} 6<\mathrm{cr}>$. The response from the compressor is $\$ \mathrm{PR} 1,079,2 \mathrm{EBD}<\mathrm{cr}>$ corresponding to pressure $\mathrm{P} 1=79 \mathrm{psig}$ and where $2 \mathrm{EBD}$ is the checksum and $\langle\mathrm{cr}\rangle$ is the carriage return.

\$STA: Read Status bits

Command with checksum and carriage return $=\$ S T A 3504<\mathrm{cr}>$

Response: \$STA, status bits, $<$ crc-16><cr>

The status bits are contained in a four character field that is the ASCII hex equivalent of a 16 bit word. For exampls status bit field of "0301" is equivalent to a binary '0000001100000001". The left most character is the MSbit. Bits ar defined as follows: 
F70 read all and command_SubVI.vi

C:\Users\LabVIEW-user\AppData\Local\Temp\F70 read all and command_SubVI.vi

Last modified on 5/20/2016 at 5:58 PM

Printed on 5/20/2016 at 5:58 PM

$\mathrm{T} 1$ through $\mathrm{T} 4$ are compressor internal temperatures in degrees $\mathrm{C}$.

$\mathrm{T} 1$ = Compressor capsule helium discharge temperature

$\mathrm{T} 2$ = Water outlet temperature

$\mathrm{T} 3=$ Water inlet temperature

$\mathrm{T} 4$ is inactive (returns "000") for most F-70 variants.

The temperature fields are always 3 characters long and are rounded to the nearest degree. Temperatures less the $100{ }^{\circ} \mathrm{C}$ have leading zeroes.

Example: $\$$ TEA, $086,040,031,000,3798<\mathrm{cr}>$ corresponding to $\mathrm{T} 1=86^{\circ} \mathrm{C}, \mathrm{T} 2=40^{\circ} \mathrm{C}$,

$\mathrm{T} 3=31^{\circ} \mathrm{C}$ and where 3798 is the checksum and $\langle\mathrm{cr}\rangle$ is the carriage return.

\$Ten: Read selected temperature $(n=1,2,3$, or 4$)$

Command with checksum and carriage return $=\$$ TE140B8 <cr $>$, $\$$ TE241F8<cr $>, \$ T E 38139<$ cr $>$, or $\$ T E 44378<$ cr $>$

Response: \$TEn,Tn, $<$ crc-16 $><c r>$

Example: host sends $\$ T E 140 B 8 c r$. The response from the compressor is $\$ T E 1,086, A D B C<C r>$ corresponding to temperature $\mathrm{T} 1=86^{\circ} \mathrm{C}$ and where $\mathrm{ADBC}$ is the checksum and $\langle\mathrm{cr}\rangle$ is the carriage return.

\$PRA: Read all pressures

Command with checksum and carriage return $=\$$ PRA95F7 $\langle\mathrm{cr}\rangle$

Response: \$PRA,P1,P2, <crc-16><cr $>$

P1 is the compressor return pressure in PSIG. P2 is inactive (returns "000") for most

F-70 variants.

The pressure fields are always 3 characters long and are rounded to the nearest whole number. Pressures less tha। 100 psig have leading zeroes.

Example: \$PRA,079,000,0CEC $<\mathrm{cr}>$ corresponding to $\mathrm{P} 1=79 \mathrm{psig}$ and where $0 \mathrm{CEC}$ is the checksum and $<\mathrm{cr}>$ is the carriage return.

\$PRn: Read selected pressure ( $n=1$ or 2 )

Command with checksum and carriage return $=\$ P R 171 \mathrm{~F} 6<\mathrm{cr}>$ or $\$ \mathrm{PR} 270 \mathrm{~B} 6<\mathrm{cr}>$

Response: \$PRn, Pn, $<$ crc-16 $>\langle$ cr $>$

Example: host sends $\$ P R 171 F 6<c r>$. The response from the compressor is $\$ P R 1,079,2 E B D<c r>$ corresponding to pressure $\mathrm{P} 1=79 \mathrm{psig}$ and where $2 \mathrm{EBD}$ is the checksum and $\langle\mathrm{cr}\rangle$ is the carriage return.

\$STA: Read Status bits

Command with checksum and carriage return $=\$ S T A 3504<\mathrm{cr}>$

Response: \$STA, status bits, <crc-16><cr>

The status bits are contained in a four character field that is the ASCII hex equivalent of a 16 bit word. For exampls status bit field of "0301" is equivalent to a binary '0000001100000001". The left most character is the MSbit. Bits ar defined as follows: 
F70 read all and command_SubVI.vi

C:\Users\LabVIEW-user\AppData\Local\Temp\F70 read all and command_SubVI.vi

Last modified on 5/20/2016 at 5:58 PM

Printed on 5/20/2016 at 5:58 PM

$\mathrm{T} 1$ through $\mathrm{T} 4$ are compressor internal temperatures in degrees $\mathrm{C}$.

$\mathrm{T} 1$ = Compressor capsule helium discharge temperature

$\mathrm{T} 2$ = Water outlet temperature

$\mathrm{T} 3=$ Water inlet temperature

T4 is inactive (returns "000") for most F-70 variants.

The temperature fields are always 3 characters long and are rounded to the nearest degree. Temperatures less the $100{ }^{\circ} \mathrm{C}$ have leading zeroes.

Example: $\$$ TEA, $086,040,031,000,3798<\mathrm{cr}>$ corresponding to $\mathrm{T} 1=86^{\circ} \mathrm{C}, \mathrm{T} 2=40^{\circ} \mathrm{C}$,

$\mathrm{T} 3=31^{\circ} \mathrm{C}$ and where 3798 is the checksum and $\langle\mathrm{cr}\rangle$ is the carriage return.

\$Ten: Read selected temperature $(n=1,2,3$, or 4$)$

Command with checksum and carriage return $=\$$ TE140B8 <cr $>, \$ T E 241 F 8<$ cr $>, \$ T E 38139<$ cr $>$, or $\$ T E 44378<$ cr $>$

Response: \$TEn,Tn, $<$ crc-16 $><c r>$

Example: host sends $\$ T E 140 B 8 \mathrm{cr}$. The response from the compressor is $\$ T E 1,086, A D B C<\mathrm{cr}>$ corresponding to temperature $\mathrm{T} 1=86^{\circ} \mathrm{C}$ and where $\mathrm{ADBC}$ is the checksum and $\langle\mathrm{cr}\rangle$ is the carriage return.

\$PRA: Read all pressures

Command with checksum and carriage return $=\$$ PRA95F7 $\langle$ cr $>$

Response: \$PRA,P1,P2, <crc-16><cr $>$

$\mathrm{P} 1$ is the compressor return pressure in PSIG. P2 is inactive (returns "000") for most

F-70 variants.

The pressure fields are always 3 characters long and are rounded to the nearest whole number. Pressures less tha। 100 psig have leading zeroes.

Example: \$PRA,079,000,0CEC $<\mathrm{cr}>$ corresponding to $\mathrm{P} 1=79 \mathrm{psig}$ and where $0 \mathrm{CEC}$ is the checksum and $<\mathrm{cr}>$ is the carriage return.

\$PRn: Read selected pressure ( $\mathrm{n}=1$ or 2 )

Command with checksum and carriage return $=\$ P R 171 \mathrm{~F} 6<\mathrm{cr}>$ or $\$ \mathrm{PR} 270 \mathrm{~B} 6<\mathrm{cr}>$

Response: \$PRn, Pn, $<$ crc-16 $>\langle$ cr $>$

Example: host sends $\$ P R 171 F 6<c r>$. The response from the compressor is $\$ P R 1,079,2 E B D<c r>$ corresponding to pressure $\mathrm{P} 1=79 \mathrm{psig}$ and where $2 \mathrm{EBD}$ is the checksum and $\langle\mathrm{cr}\rangle$ is the carriage return.

\$STA: Read Status bits

Command with checksum and carriage return $=\$ S T A 3504<\mathrm{cr}>$

Response: \$STA, status bits, $<$ crc-16><cr>

The status bits are contained in a four character field that is the ASCII hex equivalent of a 16 bit word. For exampls status bit field of "0301" is equivalent to a binary '0000001100000001". The left most character is the MSbit. Bits ar defined as follows: 
F70 read all and command_SubVI.vi

C:\Users\LabVIEW-user\AppData\Local\Temp\F70 read all and command_SubVI.vi

Last modified on 5/20/2016 at 5:58 PM

Printed on 5/20/2016 at 5:58 PM

$\mathrm{T} 1$ through $\mathrm{T} 4$ are compressor internal temperatures in degrees $\mathrm{C}$.

$\mathrm{T} 1$ = Compressor capsule helium discharge temperature

$\mathrm{T} 2$ = Water outlet temperature

$\mathrm{T} 3=$ Water inlet temperature

$\mathrm{T} 4$ is inactive (returns "000") for most F-70 variants.

The temperature fields are always 3 characters long and are rounded to the nearest degree. Temperatures less the $100{ }^{\circ} \mathrm{C}$ have leading zeroes.

Example: $\$$ TEA, $086,040,031,000,3798<\mathrm{cr}>$ corresponding to $\mathrm{T} 1=86^{\circ} \mathrm{C}, \mathrm{T} 2=40^{\circ} \mathrm{C}$,

$\mathrm{T} 3=31^{\circ} \mathrm{C}$ and where 3798 is the checksum and $\langle\mathrm{cr}\rangle$ is the carriage return.

\$Ten: Read selected temperature $(n=1,2,3$, or 4$)$

Command with checksum and carriage return $=\$$ TE140B8 <cr $>$, $\$$ TE241F8<cr $>, \$ T E 38139<$ cr $>$, or $\$ T E 44378<$ cr $>$

Response: \$TEn,Tn, $<$ crc-16 $><c r>$

Example: host sends $\$ T E 140 B 8 c r$. The response from the compressor is $\$ T E 1,086, A D B C<c r>$ corresponding to temperature $\mathrm{T} 1=86^{\circ} \mathrm{C}$ and where $\mathrm{ADBC}$ is the checksum and $\langle\mathrm{cr}\rangle$ is the carriage return.

\$PRA: Read all pressures

Command with checksum and carriage return $=\$$ PRA95F7 $\langle\mathrm{cr}\rangle$

Response: \$PRA, P1,P2, $<$ crc-16><cr $>$

P1 is the compressor return pressure in PSIG. P2 is inactive (returns "000") for most

F-70 variants.

The pressure fields are always 3 characters long and are rounded to the nearest whole number. Pressures less tha। 100 psig have leading zeroes.

Example: \$PRA,079,000,0CEC $<\mathrm{cr}>$ corresponding to $\mathrm{P} 1=79 \mathrm{psig}$ and where $0 \mathrm{CEC}$ is the checksum and $<\mathrm{cr}>$ is the carriage return.

\$PRn: Read selected pressure ( $n=1$ or 2 )

Command with checksum and carriage return $=\$ P R 171 \mathrm{~F} 6<\mathrm{cr}>$ or $\$ \mathrm{PR} 270 \mathrm{~B} 6<\mathrm{cr}>$

Response: \$PRn, Pn, $<$ crc-16 $>\langle$ cr $>$

Example: host sends $\$ P R 171 F 6<c r>$. The response from the compressor is $\$ P R 1,079,2 E B D<c r>$ corresponding to pressure $\mathrm{P} 1=79 \mathrm{psig}$ and where $2 \mathrm{EBD}$ is the checksum and $\langle\mathrm{cr}\rangle$ is the carriage return.

\$STA: Read Status bits

Command with checksum and carriage return $=\$ S T A 3504<\mathrm{cr}>$

Response: \$STA, status bits, <crc-16><cr>

The status bits are contained in a four character field that is the ASCII hex equivalent of a 16 bit word. For exampls status bit field of "0301" is equivalent to a binary '0000001100000001". The left most character is the MSbit. Bits ar defined as follows: 
F70 read all and command_SubVI.vi

C:IUsers \LabVIEW-user\AppData\Local\Temp\F70 read all and command_SubVI.vi

Last modified on 5/20/2016 at 5:58 PM

Printed on 5/20/2016 at 5:58 PM

$\mathrm{T} 1$ through $\mathrm{T} 4$ are compressor internal temperatures in degrees $\mathrm{C}$.

$\mathrm{T} 1$ = Compressor capsule helium discharge temperature

$\mathrm{T} 2$ = Water outlet temperature

$\mathrm{T} 3=$ Water inlet temperature

$\mathrm{T} 4$ is inactive (returns "000") for most F-70 variants.

The temperature fields are always 3 characters long and are rounded to the nearest degree. Temperatures less the $100{ }^{\circ} \mathrm{C}$ have leading zeroes.

Example: $\$$ TEA, $086,040,031,000,3798<\mathrm{cr}>$ corresponding to $\mathrm{T} 1=86^{\circ} \mathrm{C}, \mathrm{T} 2=40^{\circ} \mathrm{C}$,

$\mathrm{T} 3=31^{\circ} \mathrm{C}$ and where 3798 is the checksum and $\langle\mathrm{cr}\rangle$ is the carriage return.

\$Ten: Read selected temperature $(n=1,2,3$, or 4$)$

Command with checksum and carriage return $=\$$ TE140B8 <cr $>, \$ T E 241 F 8<$ cr $>, \$ T E 38139<$ cr $>$, or $\$ T E 44378<$ cr $>$

Response: \$TEn,Tn, $<$ crc-16 $><c r>$

Example: host sends $\$ T E 140 B 8 \mathrm{cr}$. The response from the compressor is $\$ T E 1,086, A D B C<\mathrm{cr}>$ corresponding to temperature $\mathrm{T} 1=86^{\circ} \mathrm{C}$ and where $\mathrm{ADBC}$ is the checksum and $\langle\mathrm{cr}\rangle$ is the carriage return.

\$PRA: Read all pressures

Command with checksum and carriage return $=\$$ PRA95F7 $\langle$ cr $>$

Response: \$PRA,P1,P2, <crc-16><cr $>$

$\mathrm{P} 1$ is the compressor return pressure in PSIG. P2 is inactive (returns "000") for most

F-70 variants.

The pressure fields are always 3 characters long and are rounded to the nearest whole number. Pressures less tha। 100 psig have leading zeroes.

Example: \$PRA,079,000,0CEC $<\mathrm{cr}>$ corresponding to $\mathrm{P} 1=79 \mathrm{psig}$ and where $0 \mathrm{CEC}$ is the checksum and $<\mathrm{cr}>$ is the carriage return.

\$PRn: Read selected pressure ( $\mathrm{n}=1$ or 2 )

Command with checksum and carriage return $=\$ P R 171 \mathrm{~F} 6<\mathrm{cr}>$ or $\$ \mathrm{PR} 270 \mathrm{~B} 6<\mathrm{cr}>$

Response: \$PRn, Pn, $<$ crc-16 $>\langle$ cr $>$

Example: host sends $\$ P R 171 F 6<c r>$. The response from the compressor is $\$ P R 1,079,2 E B D<c r>$ corresponding to pressure $\mathrm{P} 1=79$ psig and where $2 \mathrm{EBD}$ is the checksum and $\langle\mathrm{cr}\rangle$ is the carriage return. 
F70 read all and command_SubVI.vi

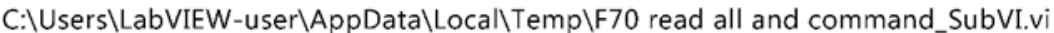

\section{Last modified on 5/20/2016 at 5:58 PM}

Printed on 5/20/2016 at 5:58 PM

Front Panel

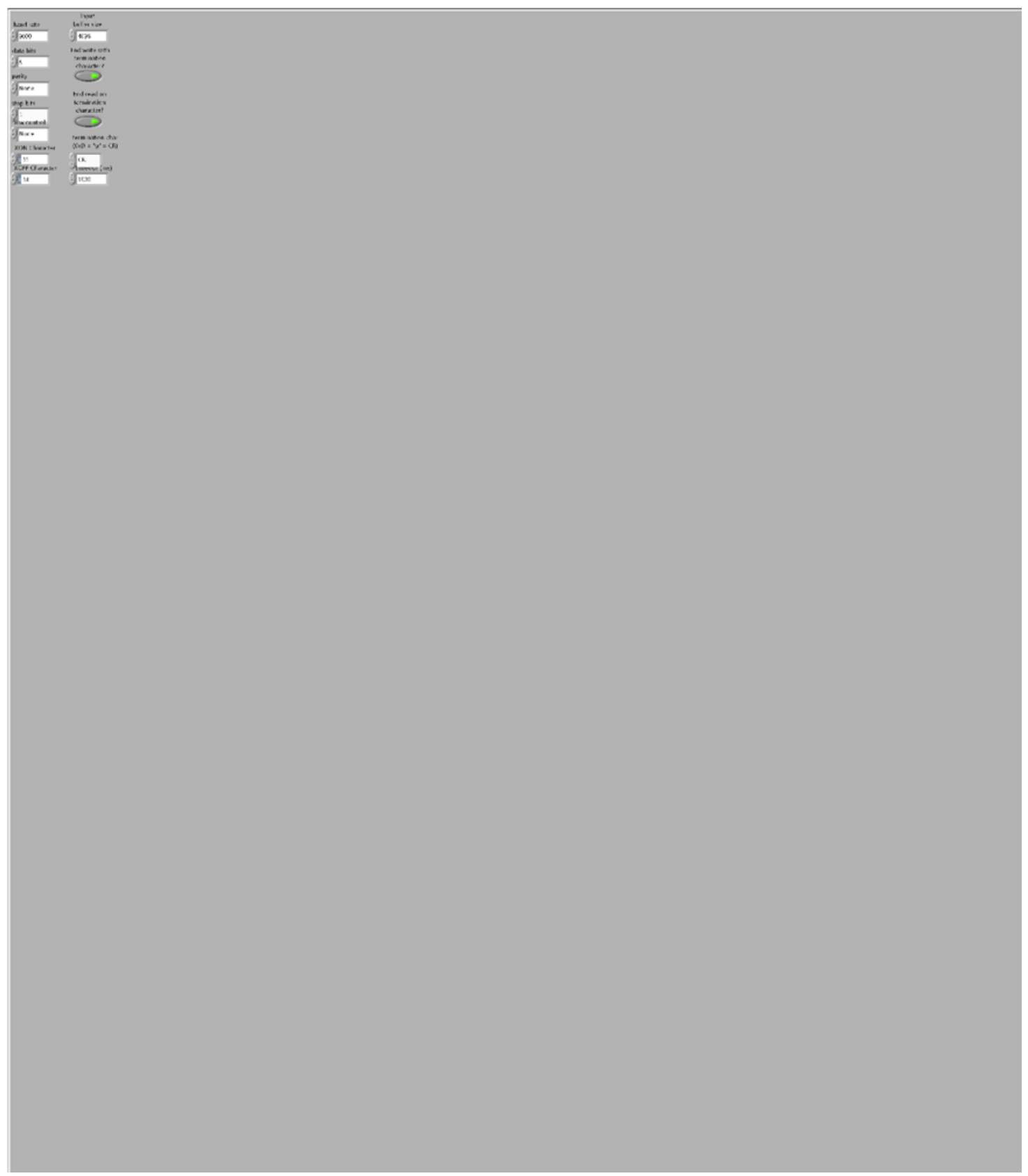


F70 read all and command_SubVI.vi

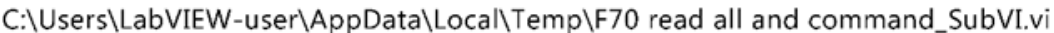
Last modified on 5/20/2016 at 5:58 PM

Printed on 5/20/2016 at 5:58 PM

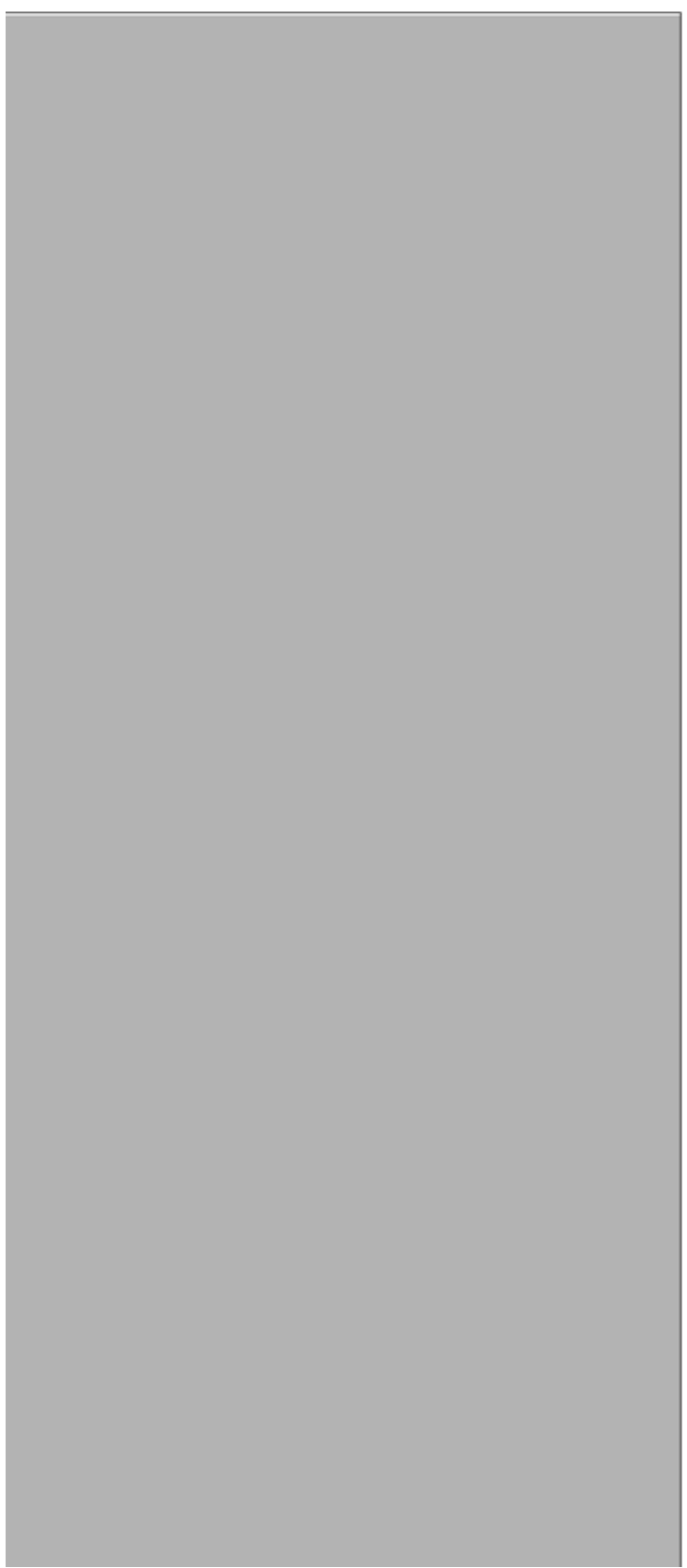


F70 read all and command_SubVI.vi

C:\Users\LabVIEW-user\AppData\Local\Temp\F70 read all and command_SubVI.vi

\section{Last modified on 5/20/2016 at 5:58 PM}

Printed on 5/20/2016 at 5:58 PM 
F70 read all and command_SubVI.vi

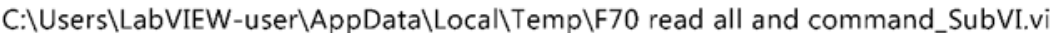
Last modified on 5/20/2016 at 5:58 PM

Printed on 5/20/2016 at 5:58 PM

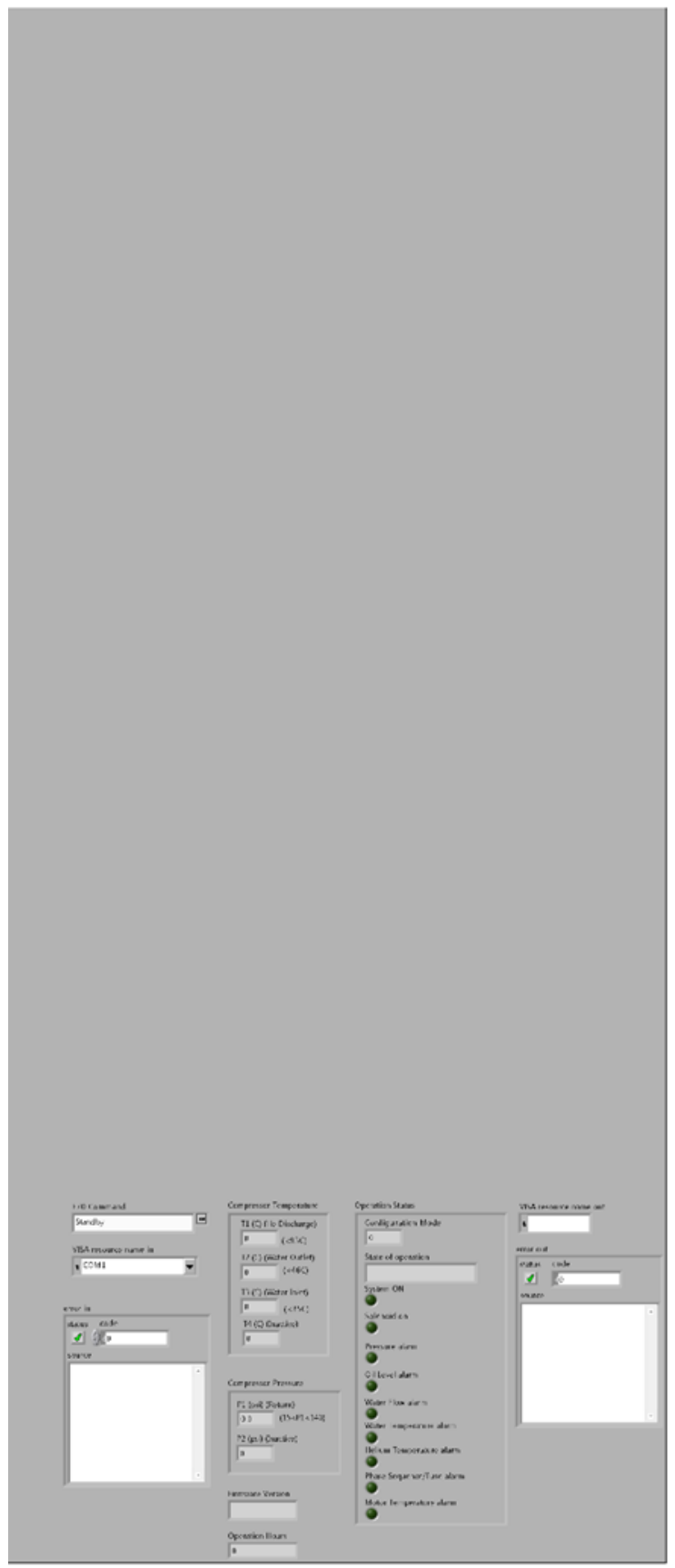


F70 read all and command_SubVI.vi

C:\Users\LabVIEW-user\AppData\Local\Temp\F70 read all and command_SubVI.vi Last modified on 5/20/2016 at 5:58 PM

Printed on 5/20/2016 at 5:58 PM

Block Diagram

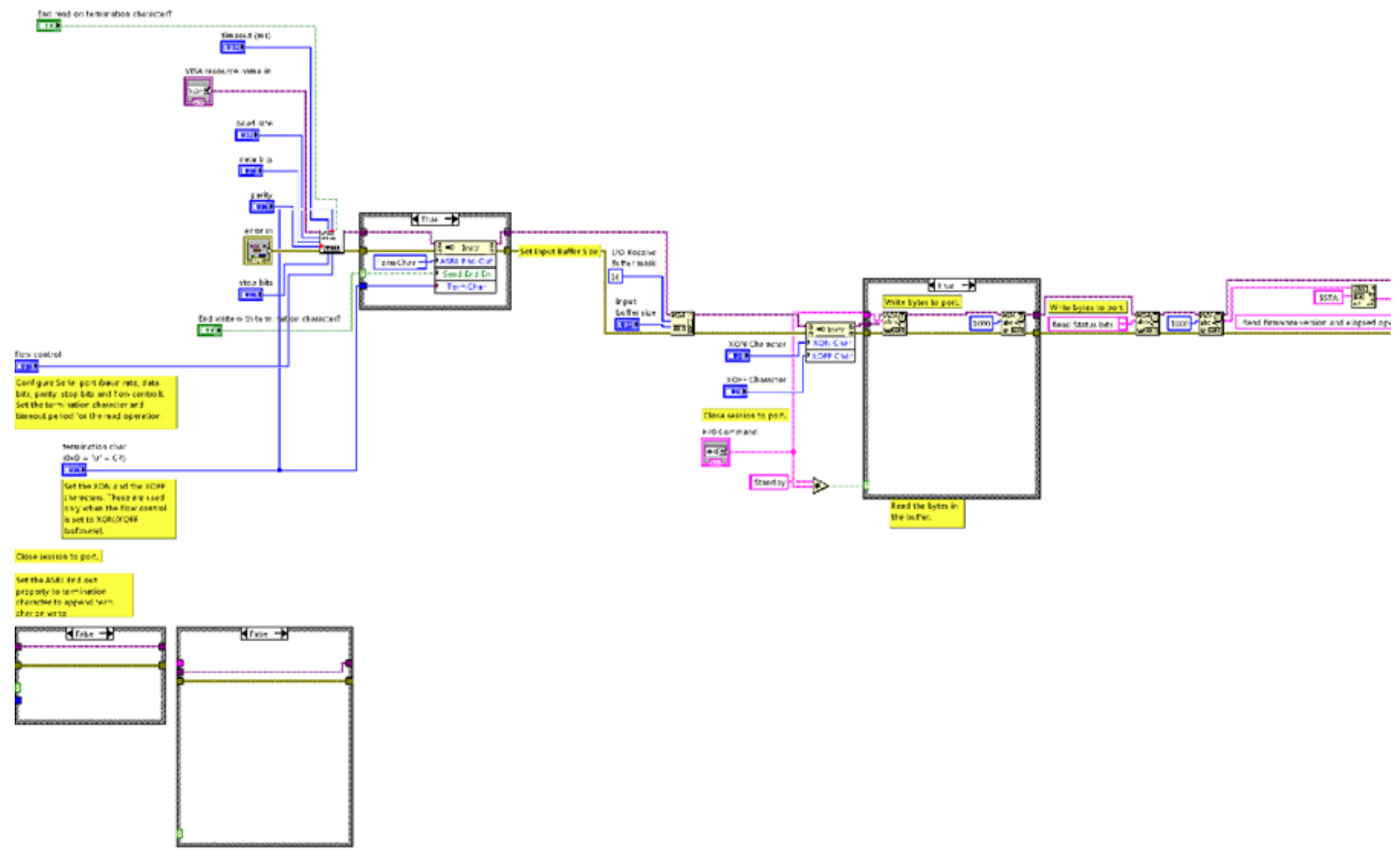


F70 read all and command_SubVI.vi

C: $\backslash$ Users $\backslash$ LabVIEW-user\AppData\Local\Temp\F70 read all and command_SubVI.vi

Last modified on 5/20/2016 at 5:58 PM

Printed on 5/20/2016 at 5:58 PM

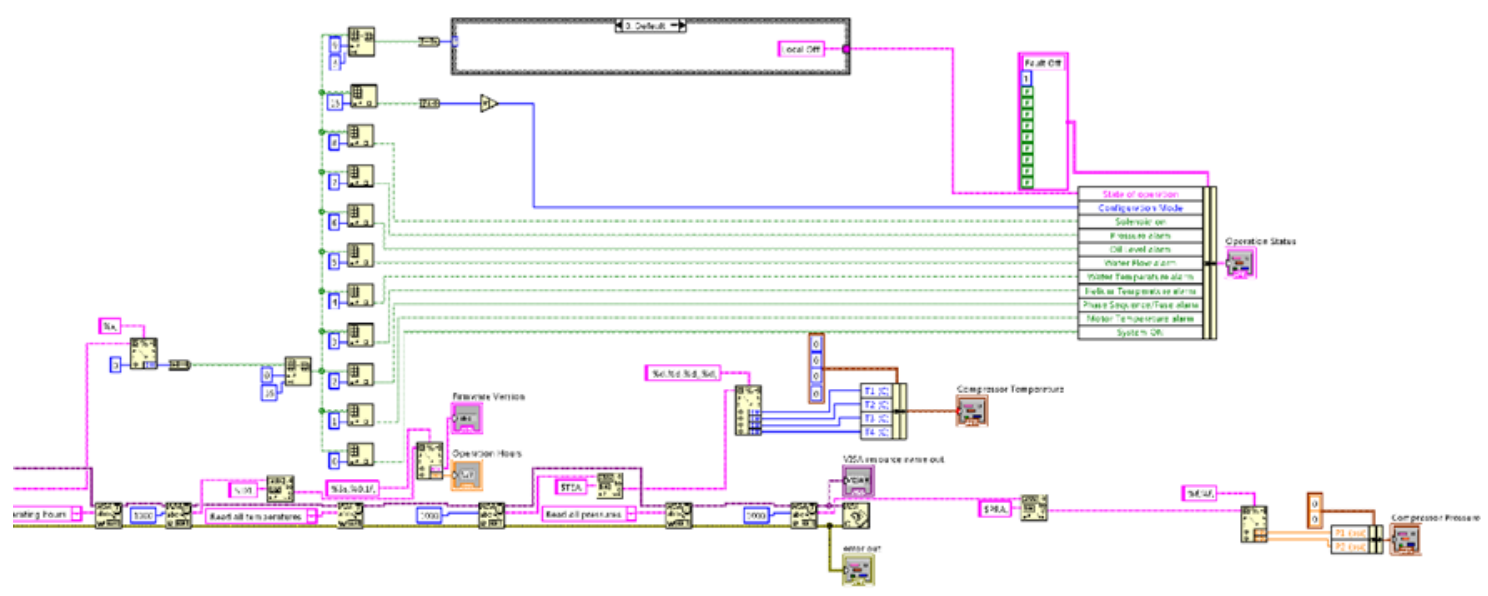

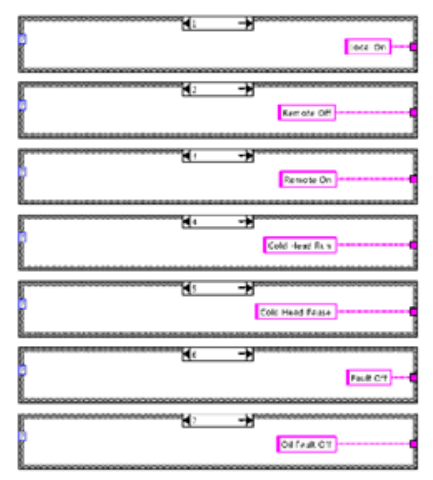


Appendix 39 Fast Sweep -Kiar v17 changed from Pseudo to differential.vi 
Fast Sweep -Kiar v17 changed from Pseudo to differential-2.vi

C:IUsers\LabVIEW-user\AppData\Local\Temp\Fast Sweep -Kiar v17 changed from Pseudo to

differential-2.vi

Last modified on 5/20/2016 at 6:05 PM

Printed on 5/20/2016 at 6:06 PM

Connector Pane

Fast Sweep -Kiar v17 changed from Pseudo to differential-2.vi

Resistor Value - Voltage on Graphene
Sample Clock Rate 1 Total Time in seconds
Max Output volage
Number of Big steps


Fast Sweep -Kiar v17 changed from Pseudo to differential-2.vi

C:\Users\LabVIEW-user\AppData\Local\Temp\Fast Sweep -Kiar v17 changed from Pseudo to differential-2.vi

Last modified on 5/20/2016 at 6:05 PM

Printed on 5/20/2016 at 6:06 PM

I. Description:

This example demonstrates how to continuously output a waveform using an internal sample clock. The automati regeneration of data has been disabled, so new data has to be provided throughout the duration of the continuous Analog Output operation. This allows the generation of any arbitrary frequency as this VI computes new data for each iteration of the loop, maintaining phase continuity of the signal.

II. Instructions for Running:

1. Select the Physical Channel to correspond to where your signal is output on the DAQ device.

2. Enter the Minimum and Maximum Voltage range.

3. Specify the desired Sample Clock Rate of the output Waveform. Higher sample clock rates will produce a smoother waveform.

4. Manually specify the Analog Output buffer size, in number of samples. A larger buffer would help nonregenerative operations avoid errors due to occasional high CPU load.

Note:

The Analog Output buffer has to be at least as big as the amount of data being written to the board per iteration. $T$ recommended buffer size would be at least twice the amount of data being written to the board per iteration.

5. Enter the Waveform Information. See the documentation of the Basic Function Generator SubVI for informatio on each parameter.

III. Block Diagram Steps:

1. Create an Analog Output Voltage channel.

2. Configure the task to prohibit the automatic regeneration of data.

3. Call the DAQmx (Sample Clock) VI to set the sample clock rate. Additionally, set the sample mode to Continuo

4. Read the actual sample clock rate (eventually coerced depending on the hardware used).

5. Compute the desired waveform, using the buffer size and the actual update rate. This VI keeps track of the phas the waveform to ensure that the generated signal is continuous.

6. Write the waveform to the output buffer.

7a. Call the Start Vl. This is only needed when the loop is executed for the first time.

7b. Do nothing.

8. Loop continuously until user presses the Stop button. Every iteration computes and writes a new waveform to 1 buffer.

9. Call the Clear Task VI to clear the Task.

10. Use the popup dialog box to display an error or warning if any.

IV. I/O Connections Overview:

Make sure your signal output terminal matches the Physical Channel I/O Control. For further connection information, refer to your hardware reference manual. 
Fast Sweep -Kiar v17 changed from Pseudo to differential-2.vi

C:IUsers\LabVIEW-user\AppData\Local\Temp\Fast Sweep -Kiar v17 changed from Pseudo to differential-2.vi

Last modified on 5/20/2016 at 6:05 PM

Printed on 5/20/2016 at 6:06 PM

\section{Front Panel}

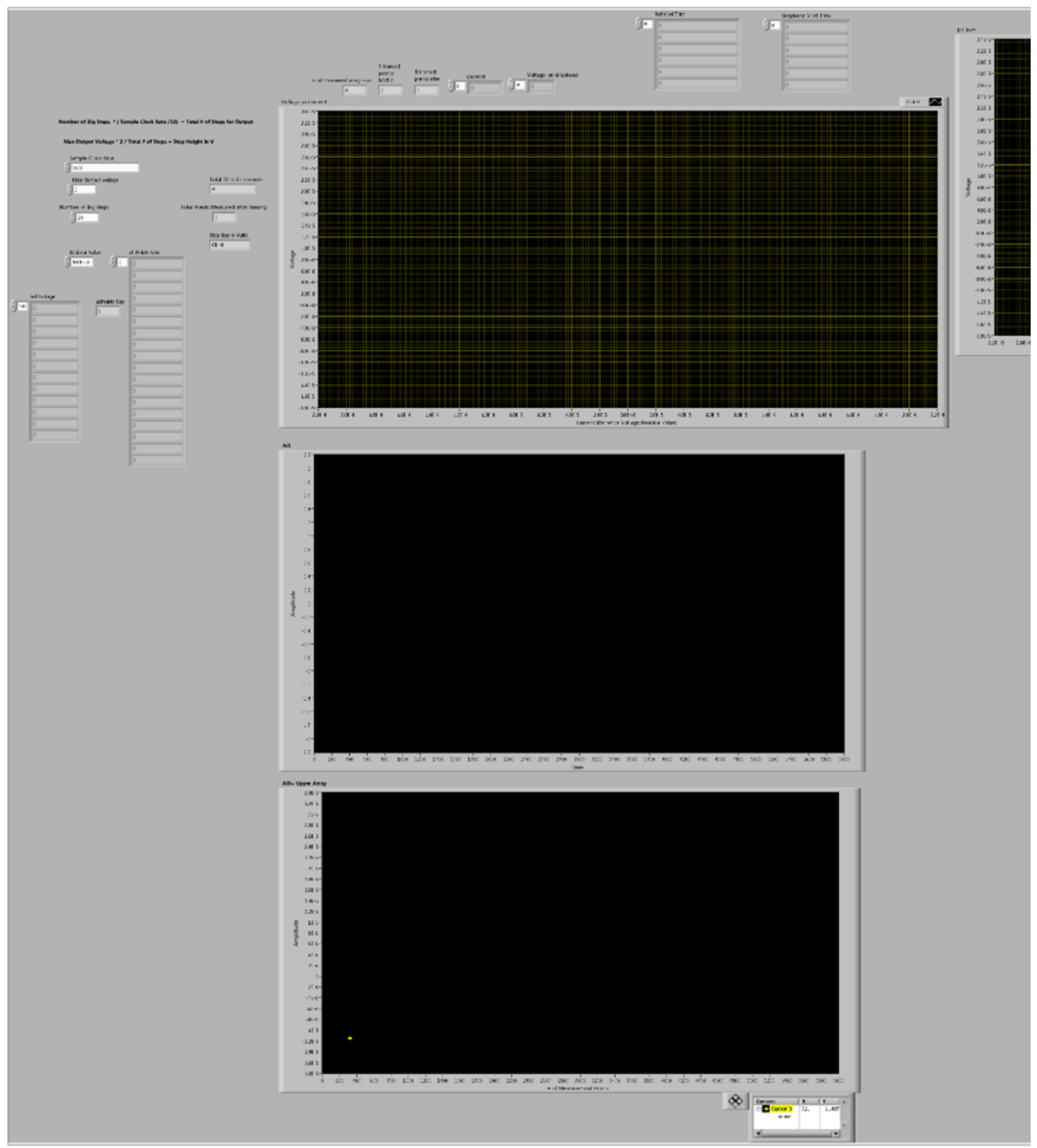


Fast Sweep -Kiar v17 changed from Pseudo to differential-2.vi

C:\Users\LabVIEW-user\AppData\Local\Temp\Fast Sweep -Kiar v17 changed from Pseudo to differential-2.vi

Last modified on 5/20/2016 at 6:05 PM

Printed on 5/20/2016 at 6:06 PM

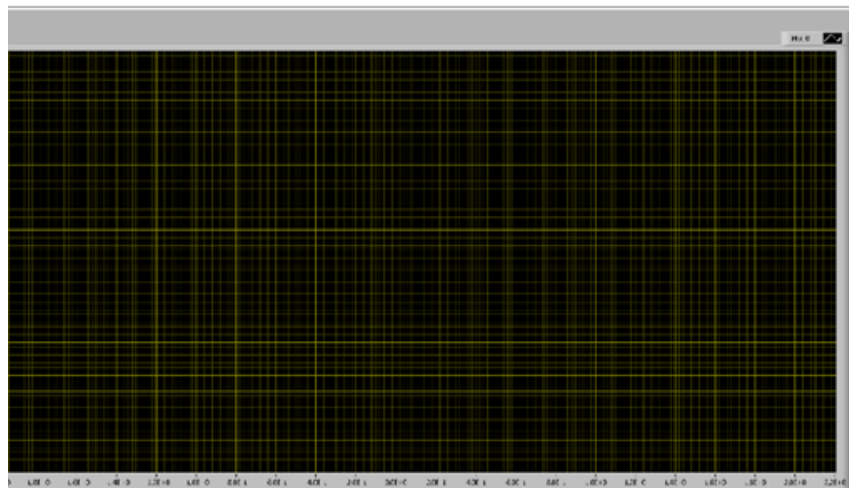


Fast Sweep -Kiar v17 changed from Pseudo to differential-2.vi

C:IUsers\LabVIEW-user\AppData\Local\Temp\Fast Sweep -Kiar v17 changed from Pseudo to differential-2.vi

Last modified on 5/20/2016 at 6:05 PM

Printed on 5/20/2016 at 6:06 PM

Block Diagram
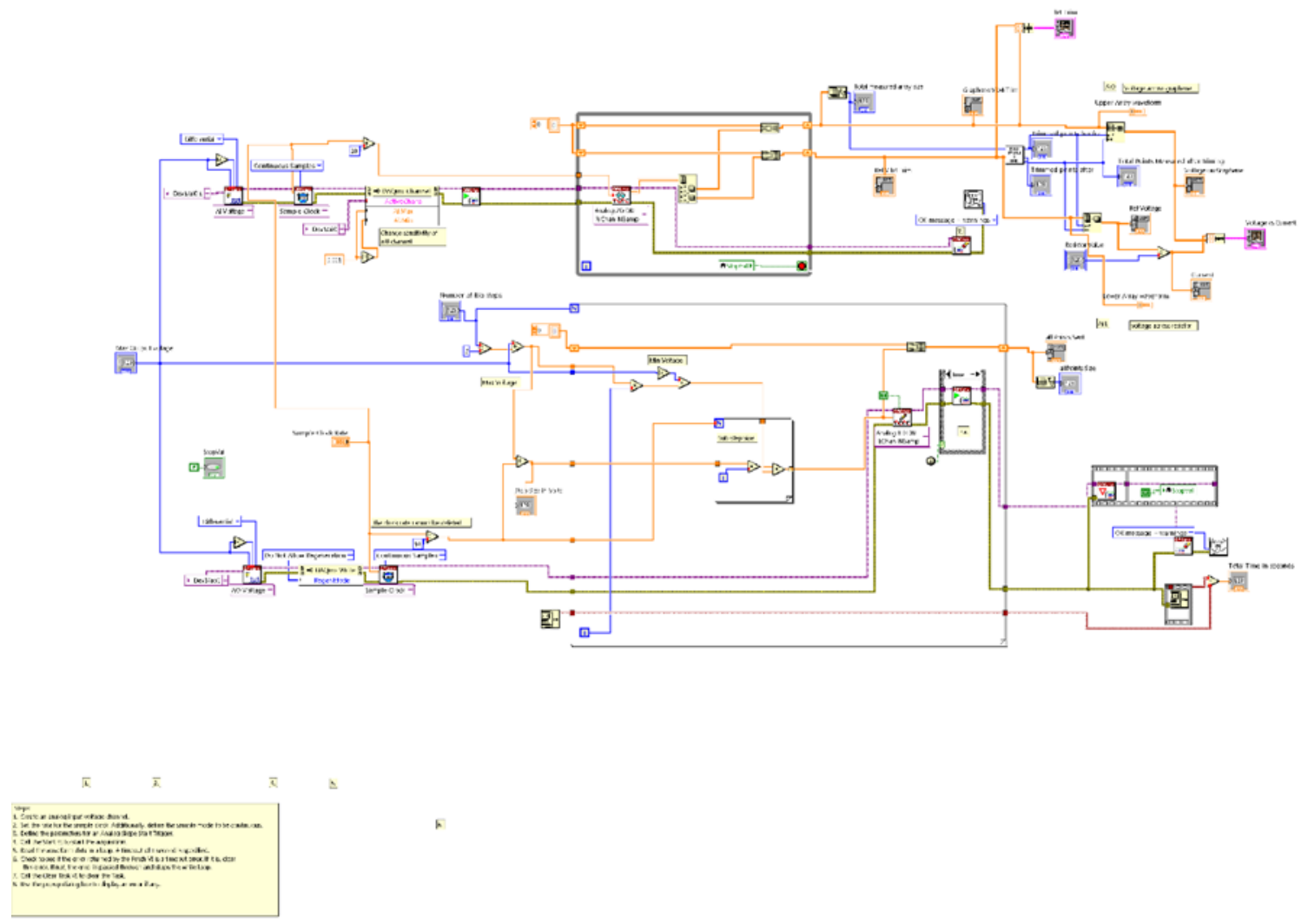
Appendix 40 Fast Sweep v7 Current step and resistor added to csv.vi 
Fast Sweep v7 Current step and resistor added to csv.vi

C:\Users\LabVIEW-user\AppData\Local\Temp\Fast Sweep v7 Current step and resistor added to csv.vi Last modified on 5/20/2016 at 6:09 PM

Printed on 5/20/2016 at 6:11 PM

Fast Sweep v7 Current step and resistor added to csv.vi

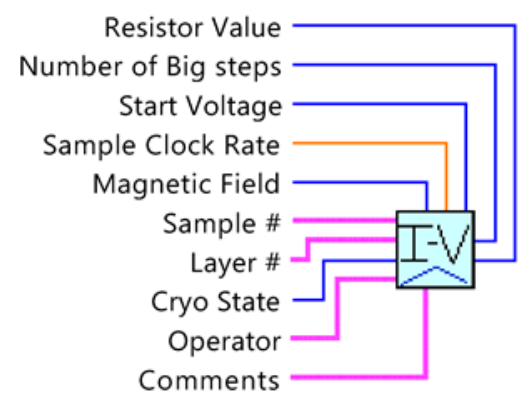

Calculates the current from the total number of steps. 
Fast Sweep v7 Current step and resistor added to csv.vi

Page 2

C:\Users\LabVIEW-user\AppData\Local\Temp\Fast Sweep v7 Current step and resistor added to csv.vi Last modified on 5/20/2016 at 6:09 PM

Printed on 5/20/2016 at 6:11 PM

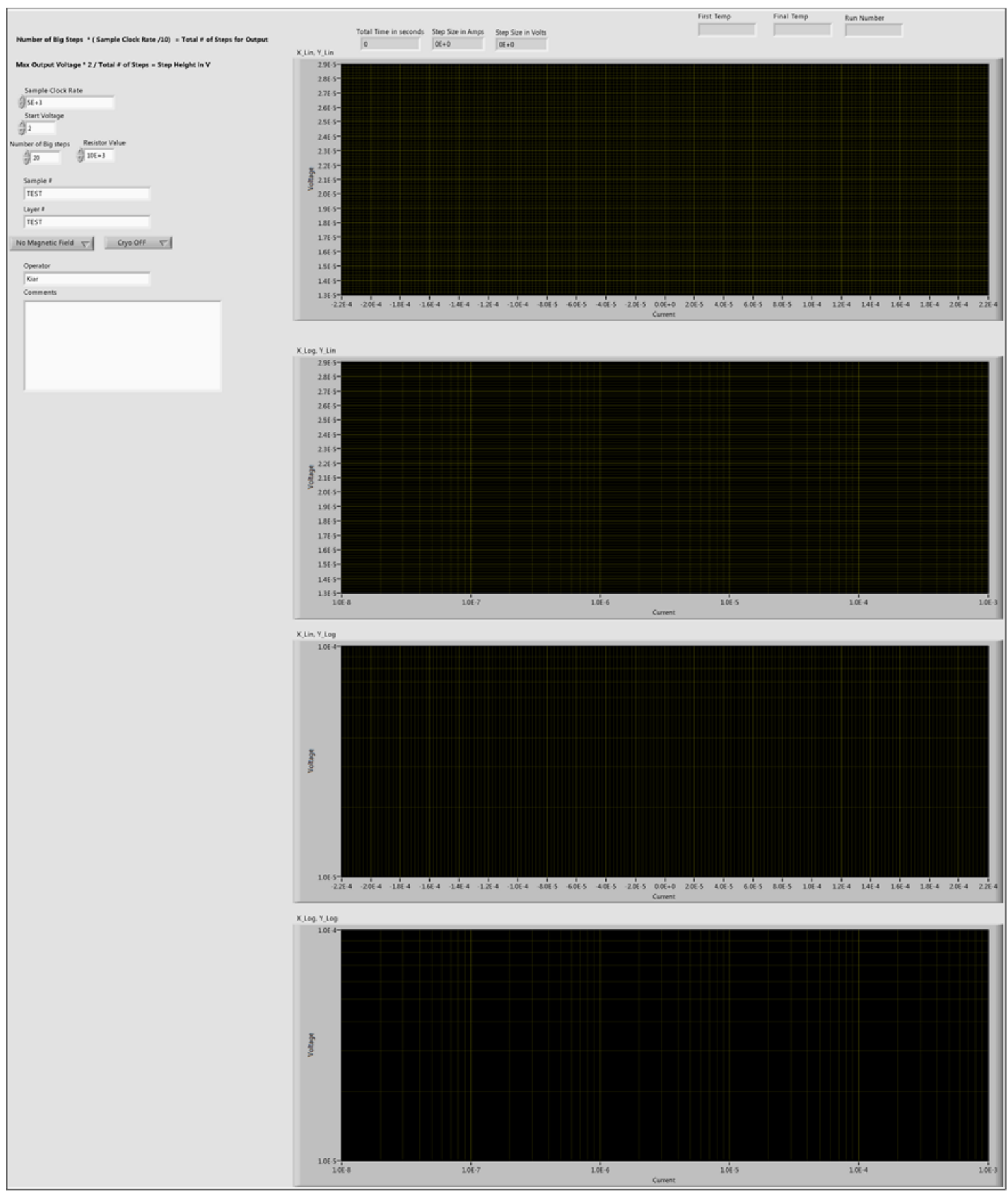


Fast Sweep v7 Current step and resistor added to csv.vi

Page 3

C:\Users\LabVIEW-user\AppData\Local\Temp\Fast Sweep v7 Current step and resistor added to csv.vi Last modified on 5/20/2016 at 6:09 PM

Printed on 5/20/2016 at 6:11 PM

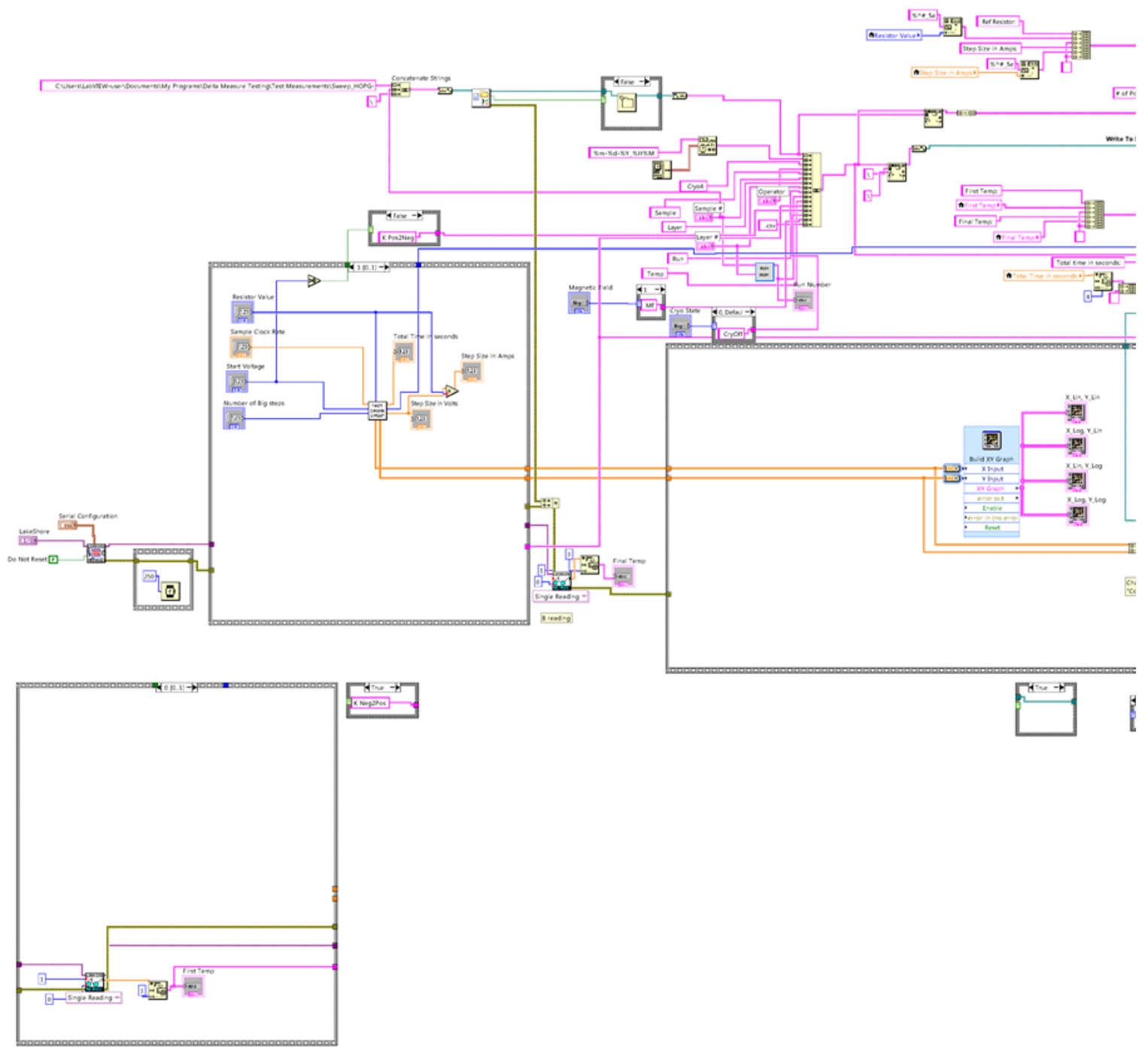

Build XY Graph

Build XY Graph

formats the data displayed on an X-Y Graph.

Convert to Dynamic Data

Convert to Dynamic Data

Converts numeric, Boolean, waveform and array data types to the dynamic data type for use with Express VIs. 
Fast Sweep v7 Current step and resistor added to csv.vi

Page 4

C:\Users\LabVIEW-user\AppData\Local\Temp\Fast Sweep v7 Current step and resistor added to csv.vi Last modified on 5/20/2016 at 6:09 PM

Printed on 5/20/2016 at 6:11 PM

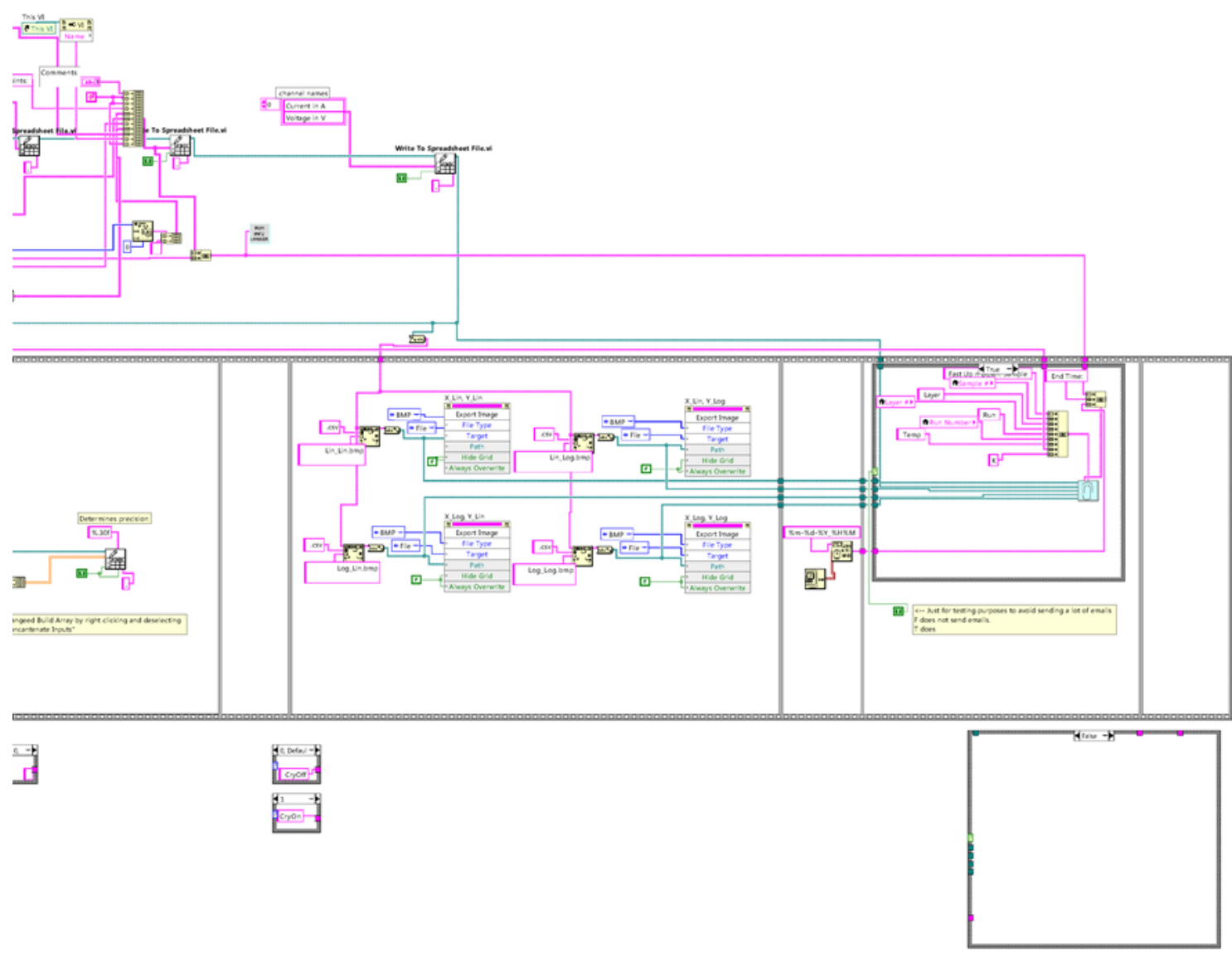


Fast Sweep v7 Current step and resistor added to csv.vi

C:\Users\LabVIEW-user\AppData\Local\Temp\Fast Sweep v7 Current step and resistor added to csv.vi Last modified on 5/20/2016 at 6:09 PM

Printed on $5 / 20 / 2016$ at $6: 11$ PM

Convert to Dynamic Data2

Convert to Dynamic Data

Converts numeric, Boolean, waveform and array data types to the dynamic data type for use with Express VIs. 
Appendix $41 \quad$ Find Start and End (SubVI).vi 
Find Start and End (SubVI).vi

C:\Users\LabVIEW-user\Desktop\KELVIN \& TUA\Kelvin\Find Start and End (SubVI).vi

Last modified on 5/20/2016 at 6:05 PM

Printed on 5/20/2016 at 6:15 PM

Find Start and End (SubVI).vi
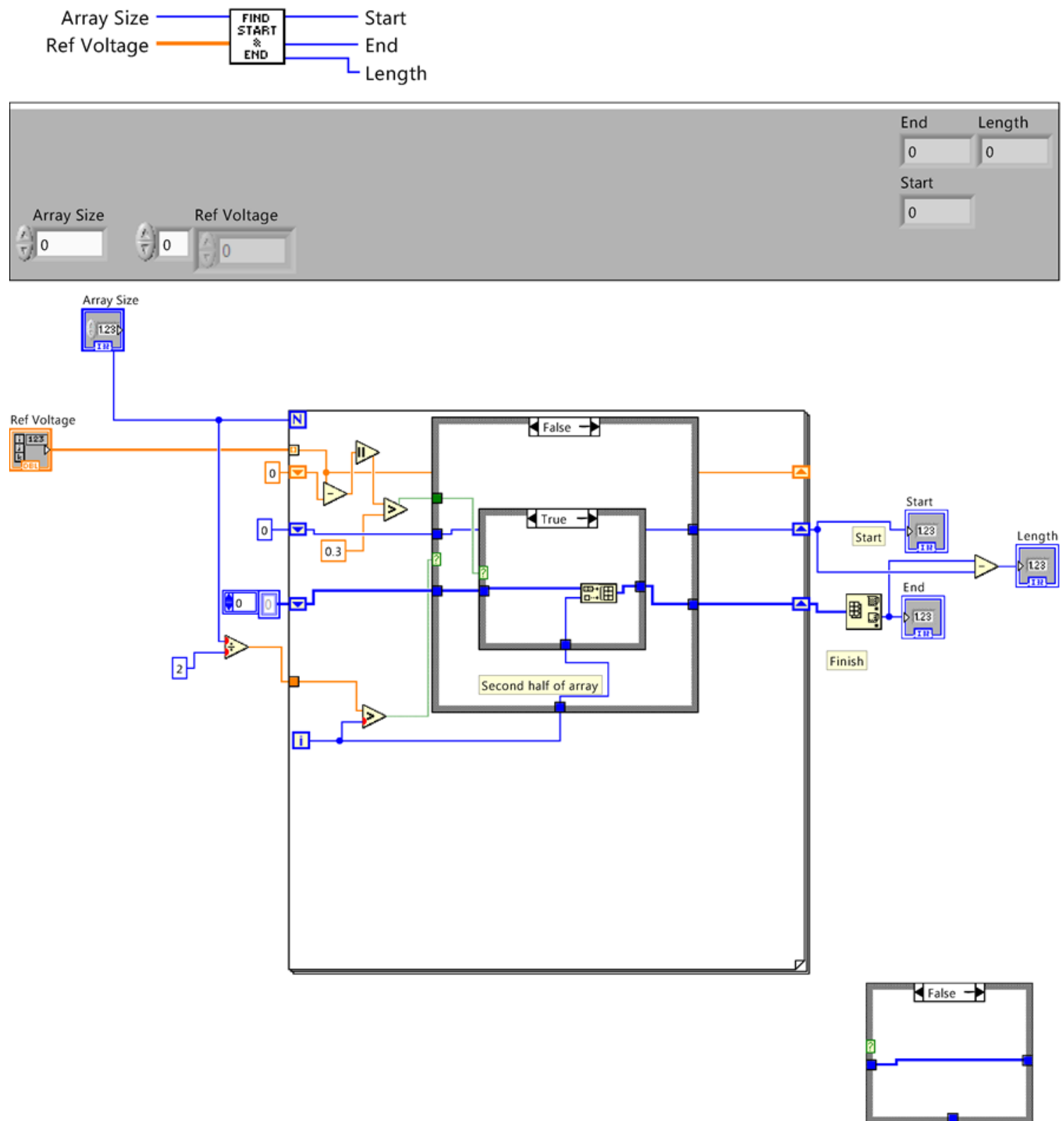
Find Start and End (SubVI).vi

Page 2 FIND

C:\Users\LabVIEW-user\Desktop\KELVIN \& TUA\Kelvin\Find Start and End (SubVI).vi Last modified on 5/20/2016 at 6:05 PM

Printed on 5/20/2016 at 6:15 PM

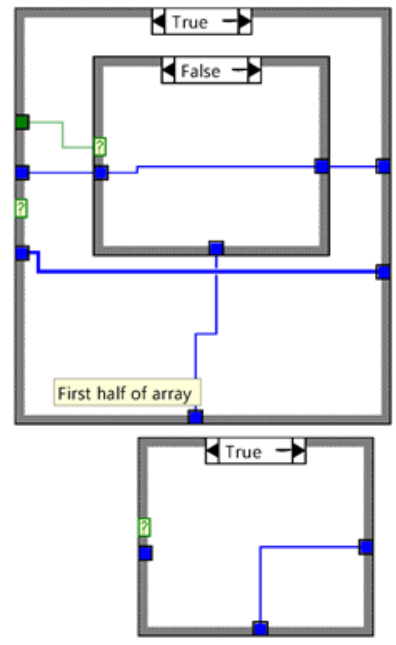


Appendix 42 GmailAttachmentSender.vi 
GmailAttachmentSender.vi

C: $\backslash$ Users $\backslash$ LabVIEW-user $\backslash A p p D a t a \backslash L o c a l \backslash T e m p \backslash G m a i l A t t a c h m e n t S e n d e r . v i$

Last modified on 5/20/2016 at 6:16 PM

Printed on $5 / 20 / 2016$ at $6: 16$ PM

\section{GmailAttachmentSender.vi}
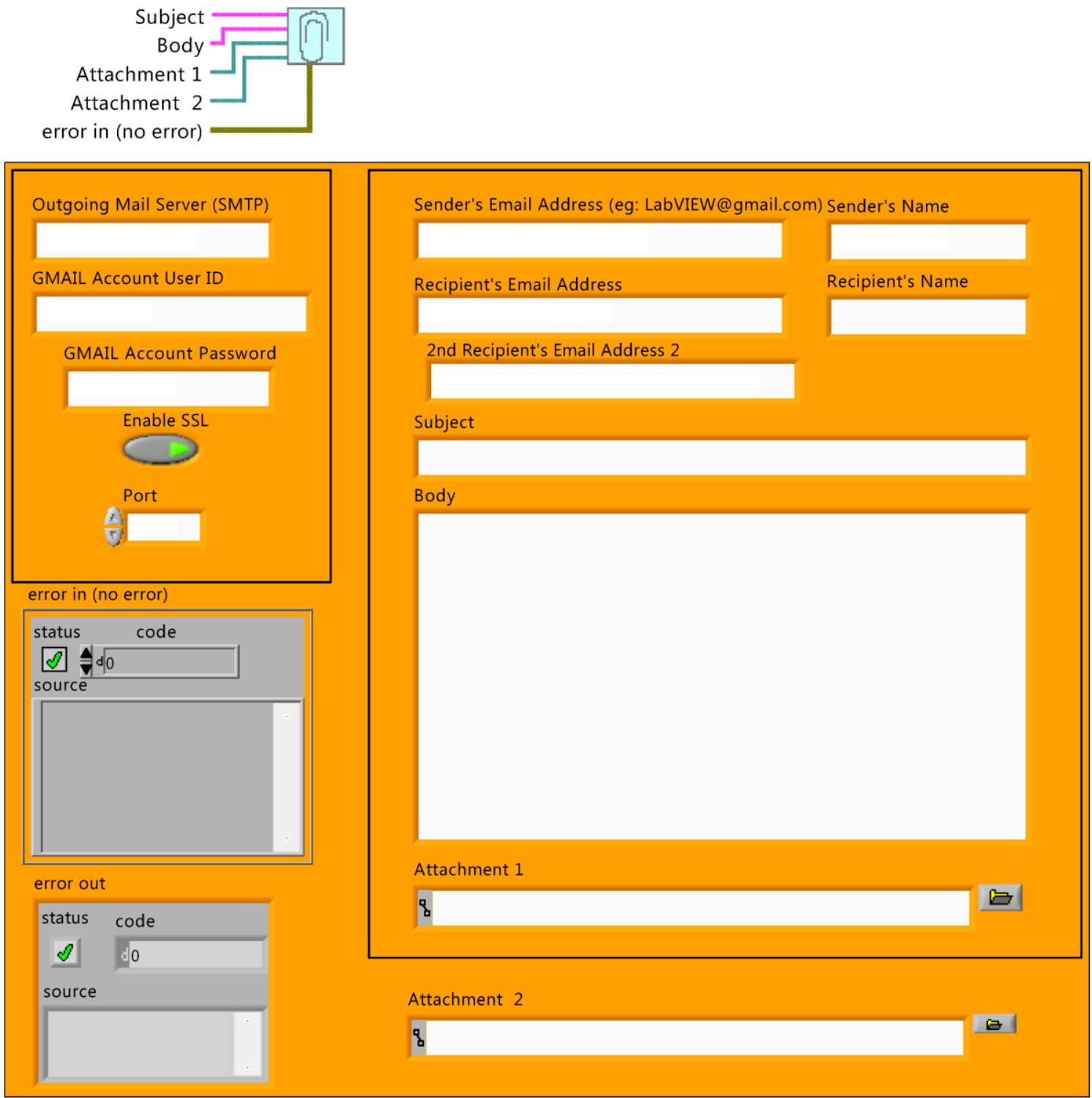
GmailAttachmentSender.vi

Page 2 [

C:\Users\LabVIEW-user\AppData\Local\Temp\GmailAttachmentSender.vi

Last modified on 5/20/2016 at 6:16 PM

Printed on 5/20/2016 at 6:16 PM

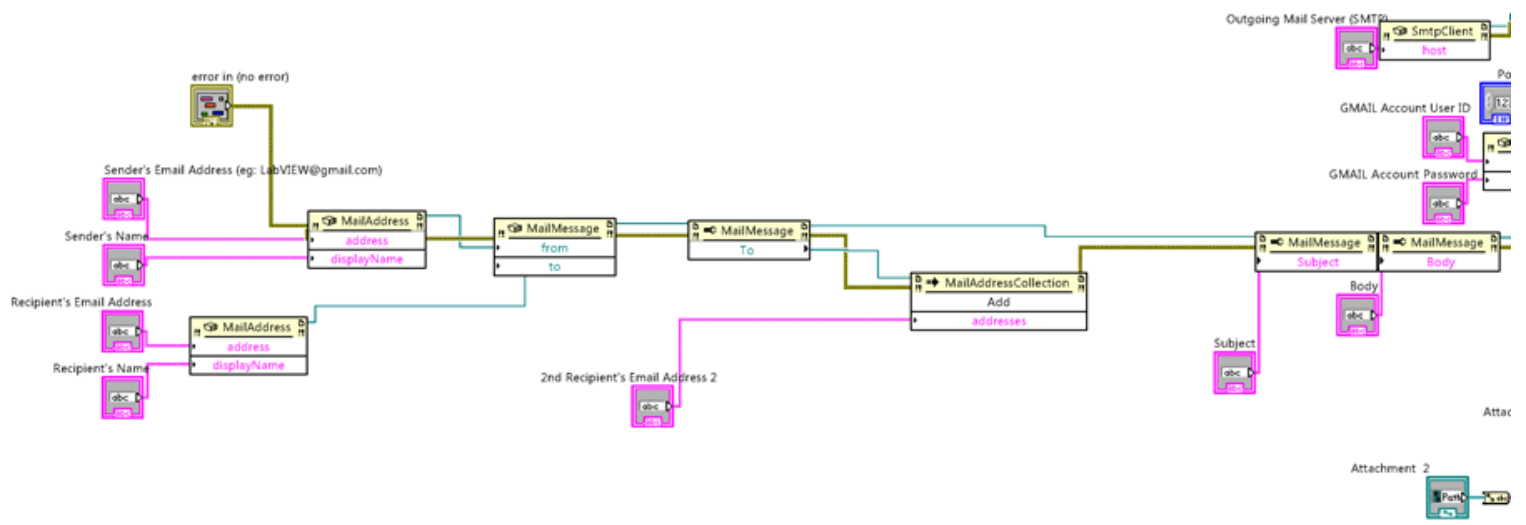


GmailAttachmentSender.vi

Page 3

C:\Users\LabVIEW-user\AppData\Local\Temp\GmailAttachmentSender.vi

Last modified on 5/20/2016 at 6:16 PM

Printed on $5 / 20 / 2016$ at $6: 16$ PM

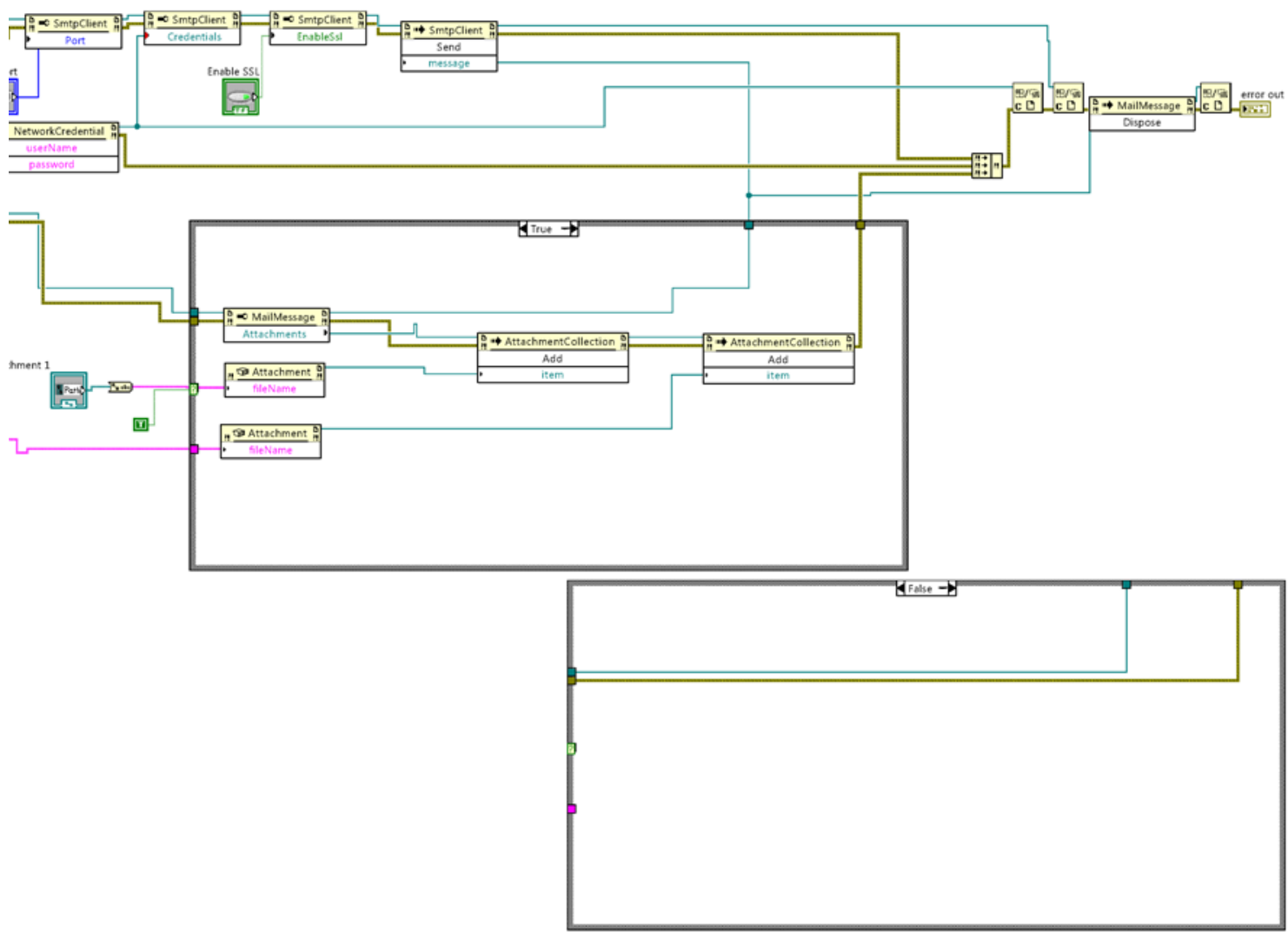


Appendix 43 Header Gen (SubVI) version 2 - run logger attempt to add

comments.vi 
Header Gen (SubVI) version 2 - run logger attempt to add comments.vi

C:\Users \LabVIEW-user\AppData\Local\Temp\Header Gen (SubVI) version 2 - run logger attempt to add comments.vi

Last modified on 5/20/2016 at 6:17 PM

Printed on 5/20/2016 at 6:19 PM

Header Gen (SubVI) version 2 - run logger attempt to add comments.vi
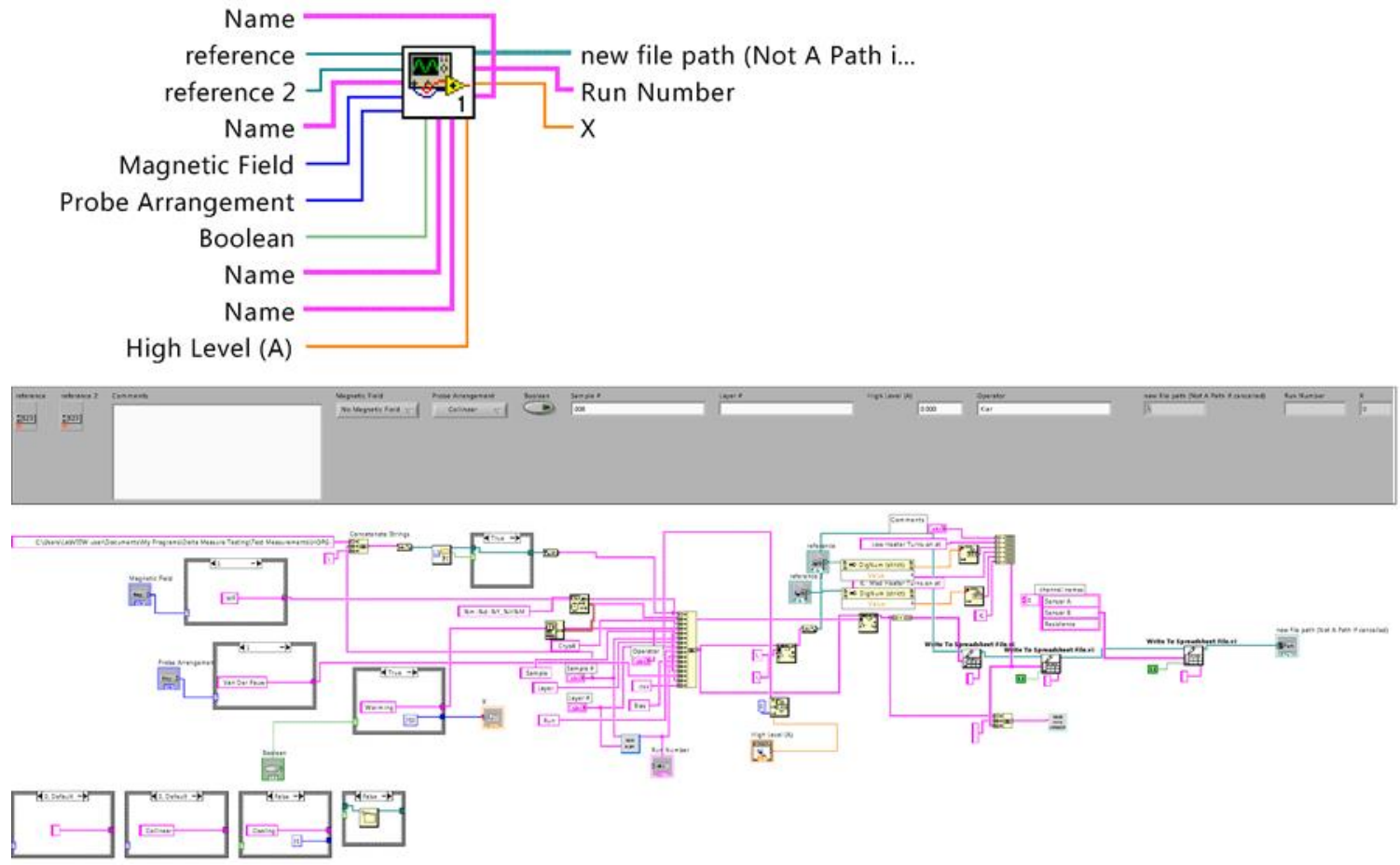
Appendix 44 Periodic Fast Sweeps Temp Dependent v2b SubVI load with

callers Default.vi 
Periodic Fast Sweeps Temp Dependent v2b SubVI load with callers Default.vi

C:\Users\LabVIEW-user\AppData\Local\Temp\Periodic Fast Sweeps Temp Dependent v2b SubVI load with callers Default.vi

Last modified on 5/20/2016 at 6:19 PM

Printed on 5/20/2016 at 6:20 PM

Periodic Fast Sweeps Temp Dependent v2b SubVI load with callers Default.vi
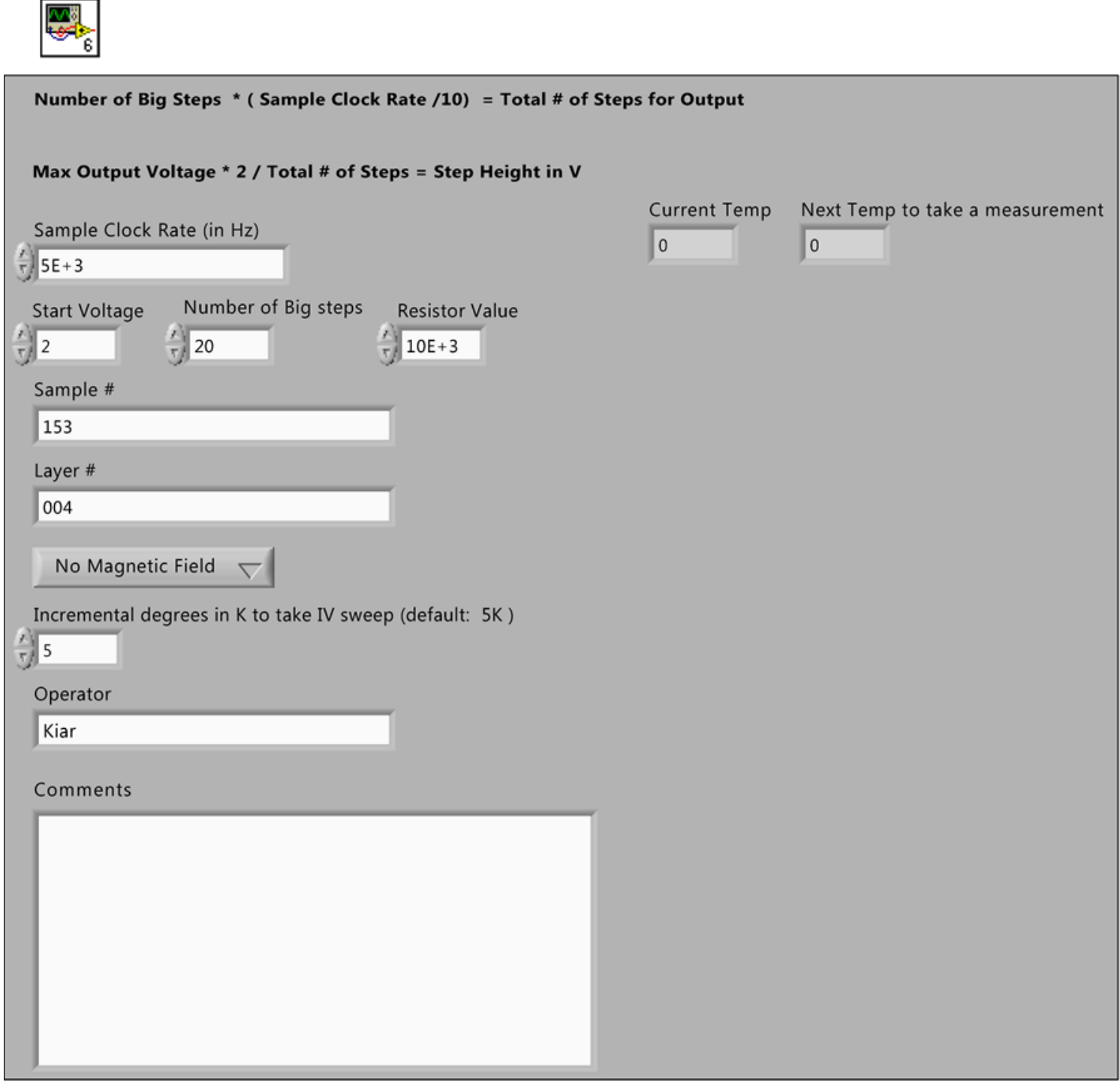
Periodic Fast Sweeps Temp Dependent v2b SubVI load with callers Default.vi

C:\Users \LabVIEW-user $\backslash A p p D a t a \backslash L o c a l \backslash T e m p \backslash P e r i o d i c$ Fast Sweeps Temp Dependent v2b SubVI load with callers Default.vi

Last modified on 5/20/2016 at 6:19 PM

Printed on 5/20/2016 at 6:20 PM

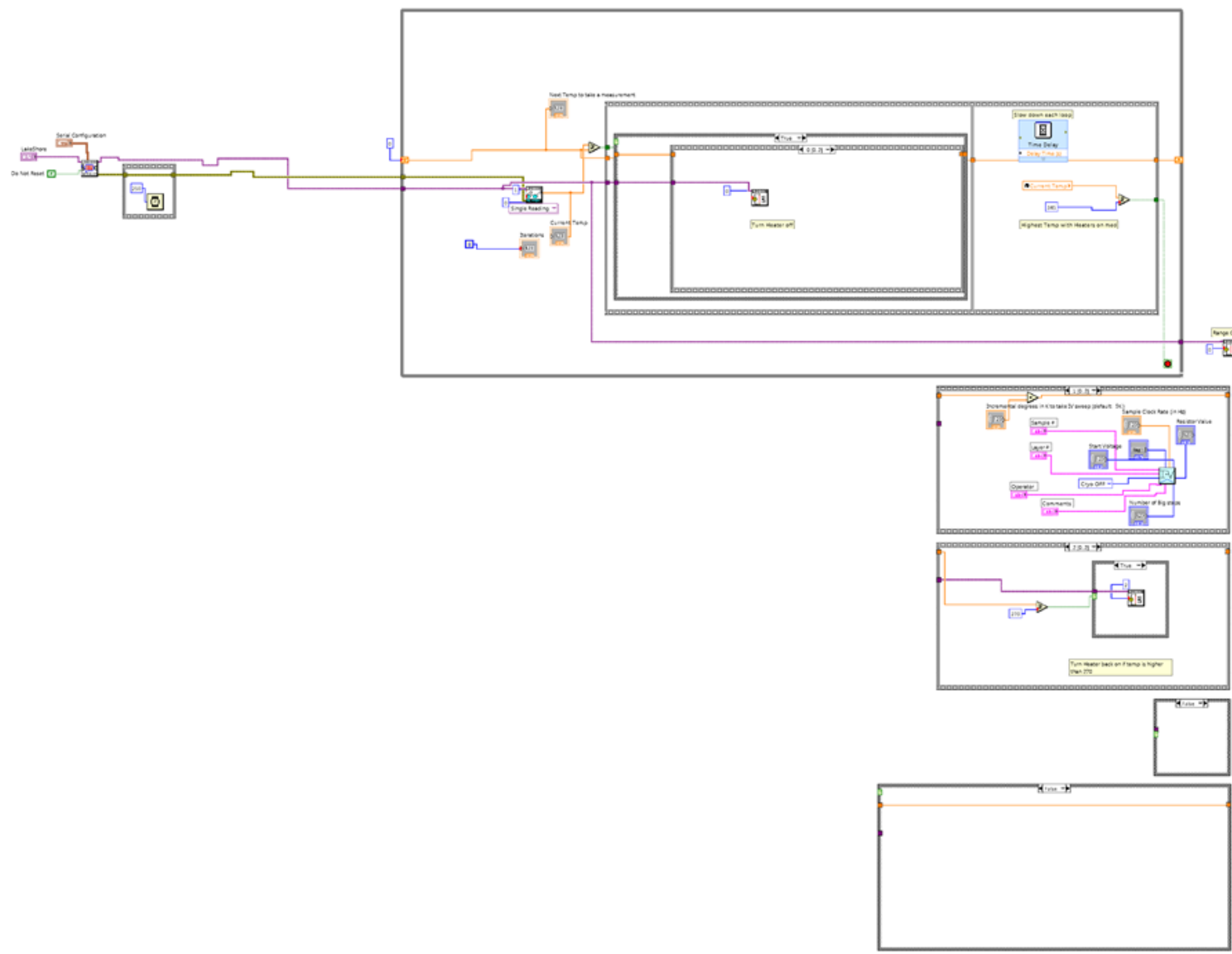

区 Time Delay

Time Delay

Inserts a time delay into the calling VI.

This Express VI is configured as follows:

Delay Time: $20 \mathrm{~s}$ 
Appendix 45 Periodic Sweeps Temp Dependent v4c - Neg to Pos - Num runs

calculated.vi 
Periodic Sweeps Temp Dependent v4c - Neg to Pos - Num runs calculated.vi

C:\Users\LabVIEW-user\AppData\Local\Temp\Periodic Sweeps Temp Dependent v4c - Neg to Pos -

Num runs calculated.vi

Last modified on $5 / 20 / 2016$ at 6:21 PM

Printed on 5/20/2016 at 6:23 PM

Periodic Sweeps Temp Dependent v4c - Neg to Pos - Num runs calculated.vi
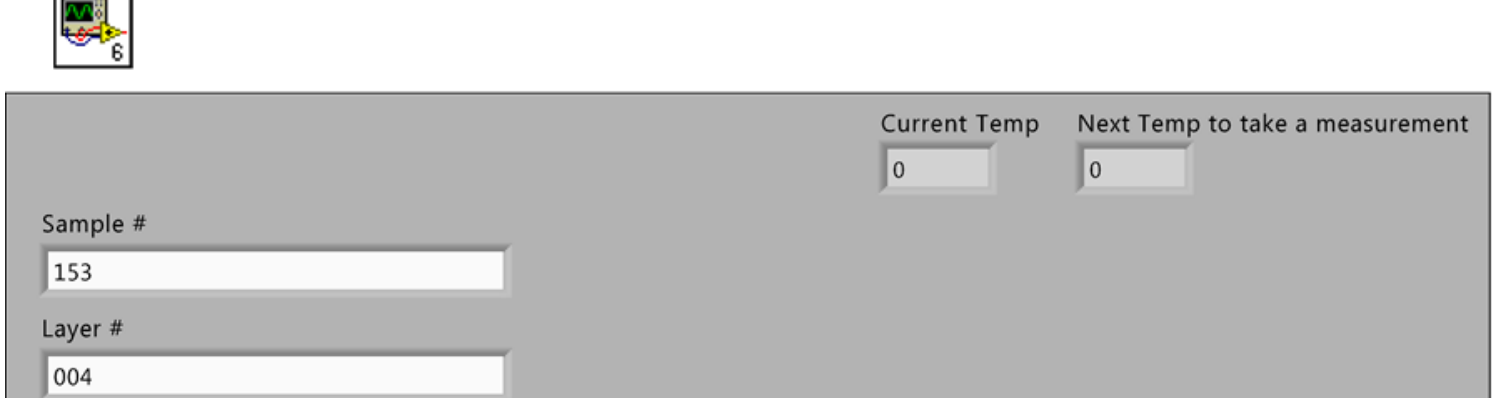

Incremental degrees in $\mathrm{K}$ to take IV sweep (default: $25 \mathrm{~K}$ )

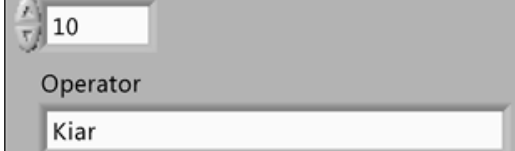

Comments

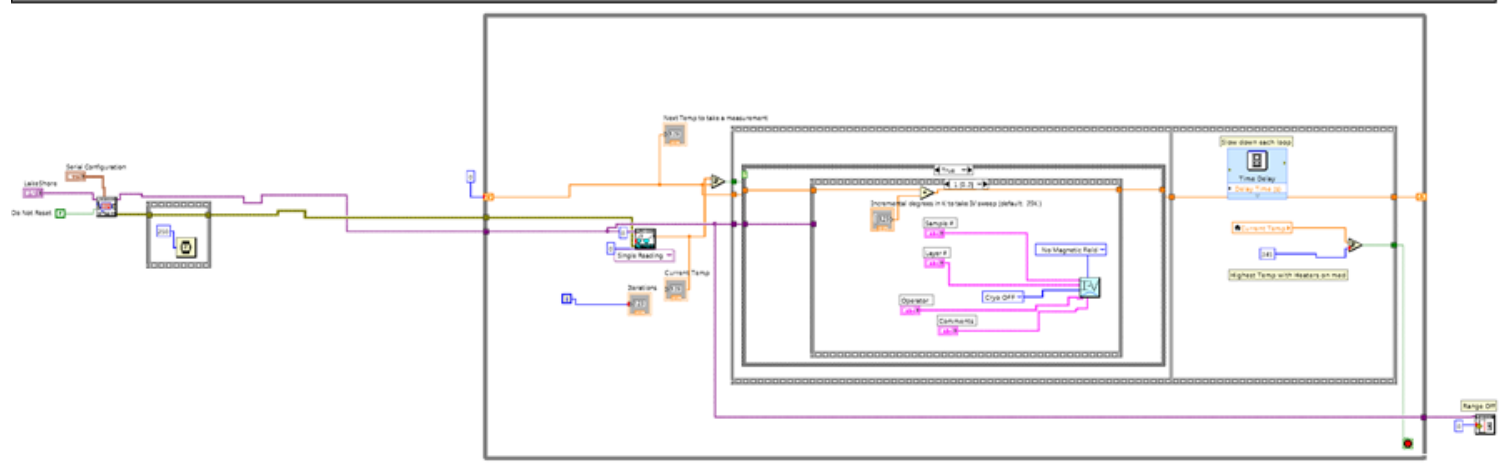


Periodic Sweeps Temp Dependent v4c - Neg to Pos - Num runs calculated.vi C:\Users\LabVIEW-user\AppData\Local\Temp\Periodic Sweeps Temp Dependent v4c - Neg to Pos Num runs calculated.vi

Last modified on $5 / 20 / 2016$ at 6:21 PM

Printed on 5/20/2016 at 6:23 PM

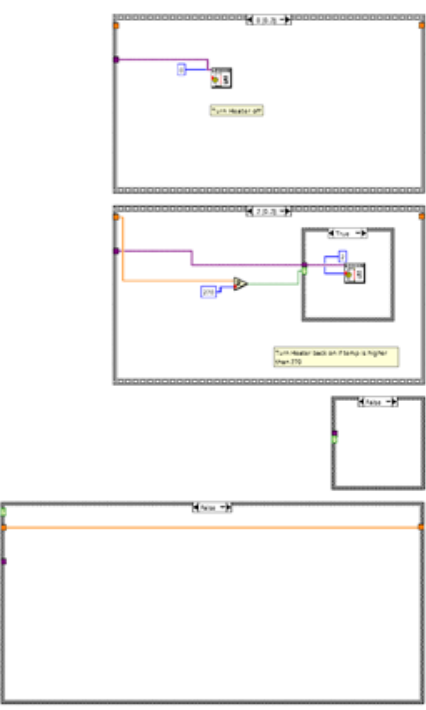

邓 Time Delay

Time Delay

Inserts a time delay into the calling VI.

-

This Express VI is configured as follows:

Delay Time: $20 \mathrm{~s}$ 
Appendix $46 \quad$ RunCalculatorINI.vi 
RunCalculatorINI.vi

C:\Users \LabVIEW-user $\backslash A p p D a t a \backslash L o c a l \backslash T e m p \backslash R u n C a l c u l a t o r I N I . v i$

Last modified on 5/20/2016 at 6:24 PM

Printed on 5/20/2016 at 6:24 PM

\section{RunCalculatorINI.vi}

\begin{tabular}{|c|c|c|}
\hline Sample & RUN & Run Number \\
\hline Laye & NUM & $\begin{array}{l}\text { file created? } \\
\text { found? }\end{array}$ \\
\hline
\end{tabular}

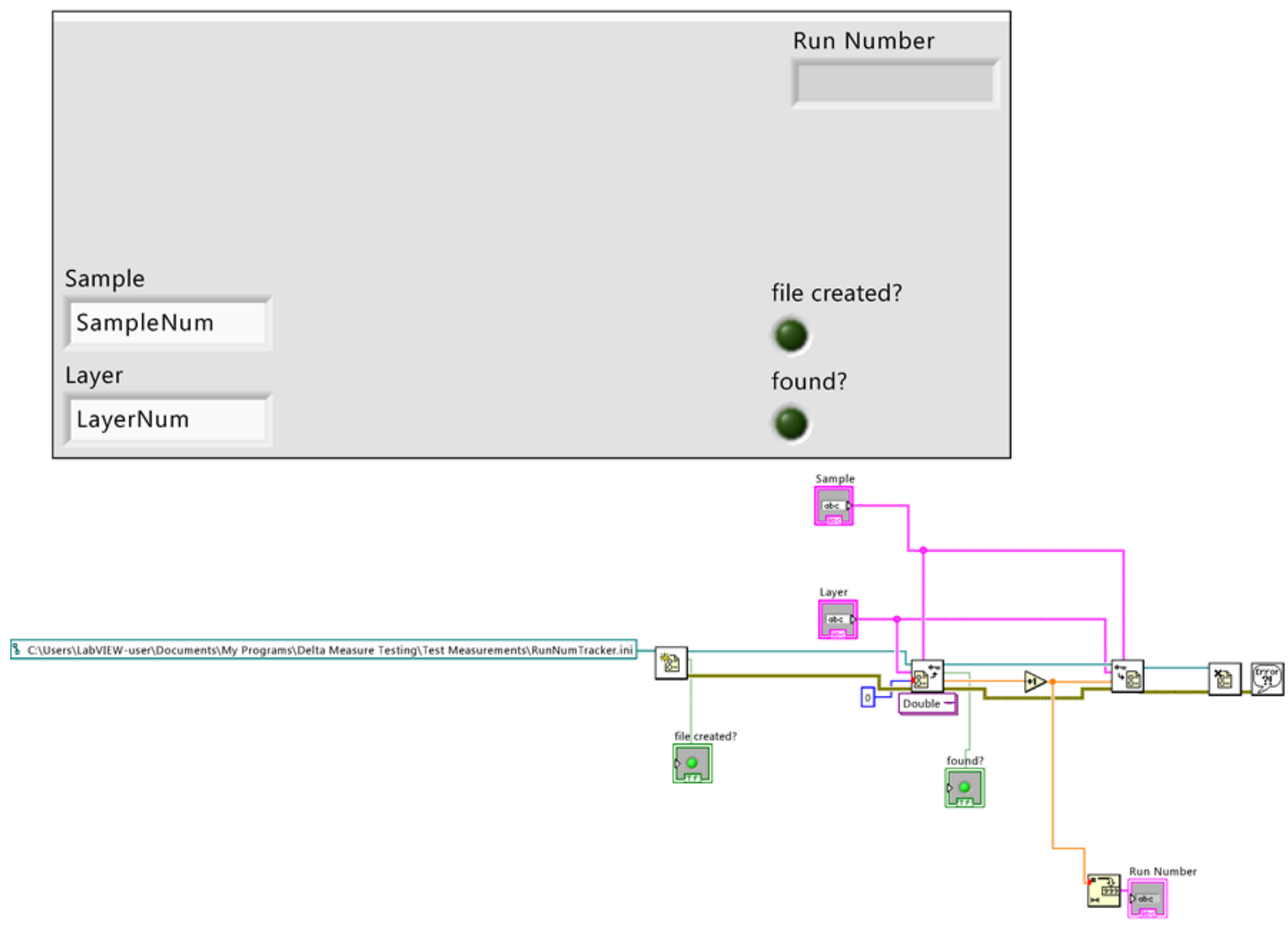


Appendix 47 RunInfoLogger.vi 
RunInfoLogger.vi

C:\Users\LabVIEW-user \AppData\Local\Temp\RunInfoLogger.vi

Last modified on 5/20/2016 at 6:24 PM

Printed on 5/20/2016 at 6:25 PM

RunInfologger.vi

Run Info $\longrightarrow \underset{\substack{\text { RUN } \\ \text { INFO } \\ \text { LOGGER }}}{\text { RUT }}$

Run Info

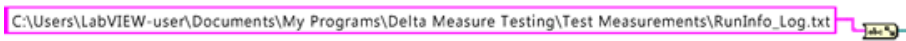

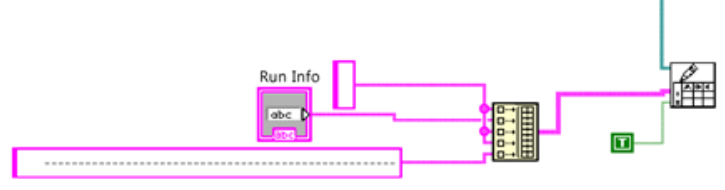


Appendix $48 \quad$ SubVI for $\mathrm{R}$ v $\mathrm{T}$ run v3 - Case structure removed.vi 
Sub VI for R v T run v3 - Case structure removed.vi

Page 1

C:\Users \LabVIEW-user \AppData\Local\Temp\Sub VI for R v T run v3 - Case structure removed.vi Last modified on 5/20/2016 at 6:25 PM

Printed on 5/20/2016 at 6:25 PM

Sub VI for R v T run v3 - Case structure removed.vi

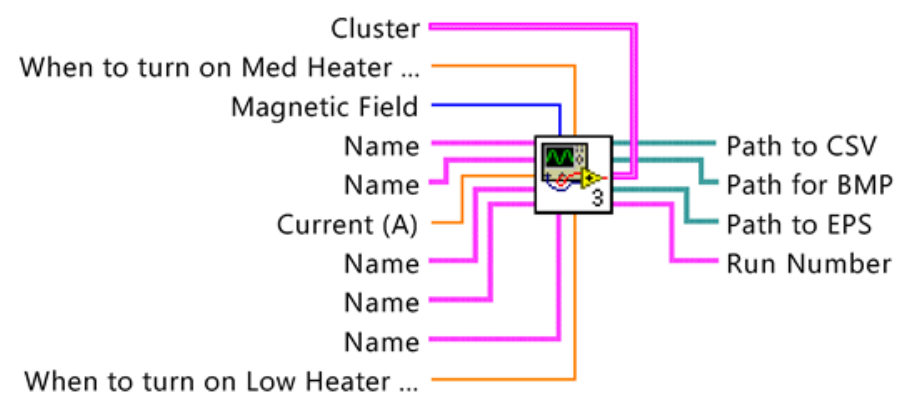

When to turn on Low Heater ... 
Sub VI for R v T run v3 - Case structure removed.vi

Page 2 国

C:\Users \LabVIEW-user \AppData\Local\Temp\Sub VI for R v T run v3 - Case structure removed.vi Last modified on 5/20/2016 at 6:25 PM

Printed on 5/20/2016 at 6:25 PM

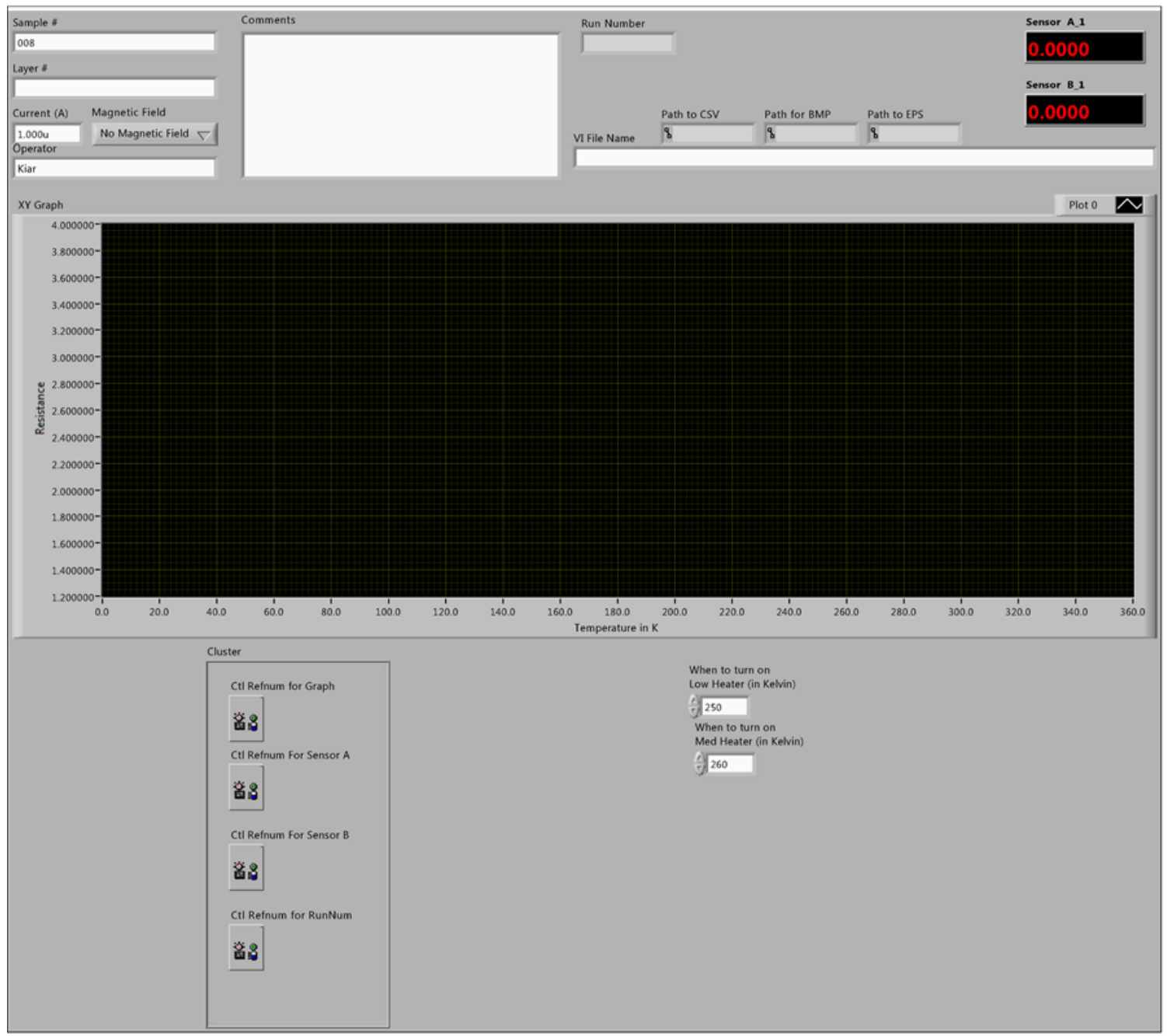


Sub VI for R v T run v3 - Case structure removed.vi

C: $\backslash$ Users $\backslash$ LabVIEW-user $\backslash A p p D a t a \backslash L o c a l \backslash T e m p \backslash S u b$ VI for $R \vee T$ run v3 - Case structure removed.vi

Last modified on 5/20/2016 at 6:25 PM

Printed on 5/20/2016 at 6:25 PM
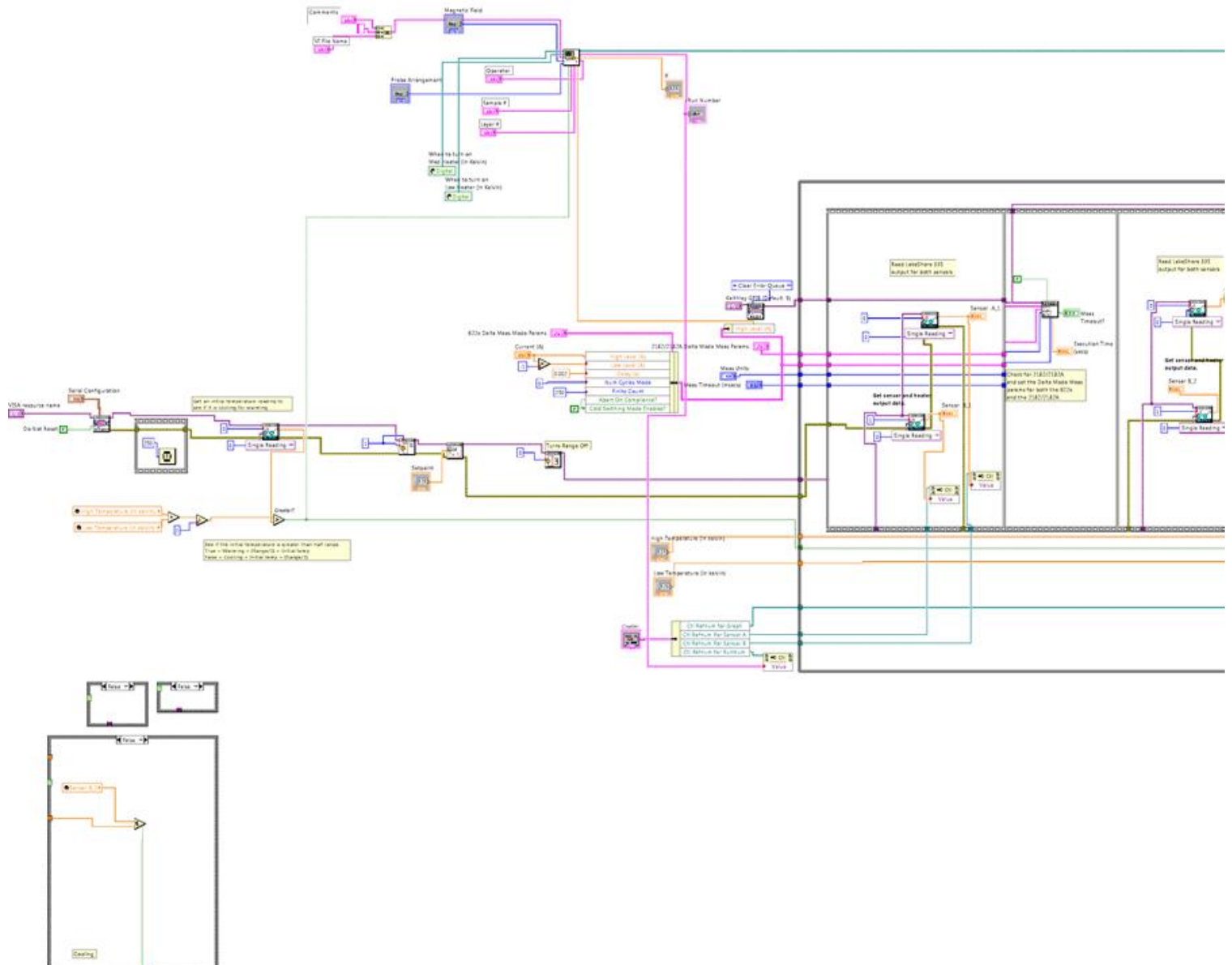

Build XY Graph

Build XY Graph

formats the data displayed on an X-Y Graph.

Convert from Dynamic Data

Convert from Dynamic Data

Converts the dynamic data type to numeric, Boolean, waveform, and array data types for use with other VIs and functions. 
Sub VI for R v T run v3 - Case structure removed.vi

C:\Users \LabVIEW-user \AppData\Local\Temp\Sub VI for R v T run v3 - Case structure removed.vi Last modified on 5/20/2016 at 6:25 PM

Printed on $5 / 20 / 2016$ at $6: 25$ PM

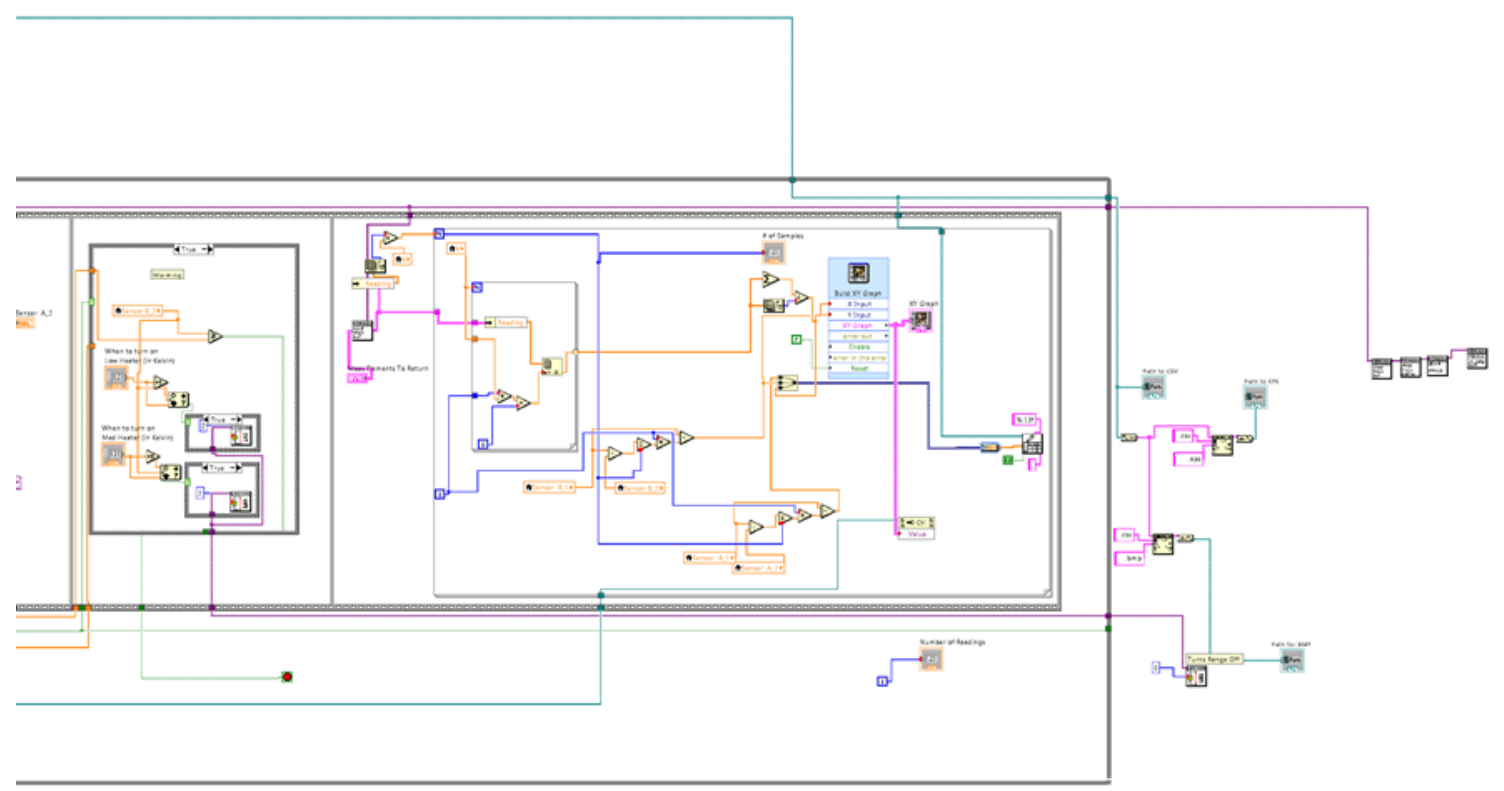


Appendix 49 Sweep Up and Down v5e - Neg to Pos - MF selection and

\author{
CryoState added.vi
}


Sweep Up and Down v5e - Neg to Pos - MF selection and CryoState added..vi

Page $1 \cong-V$

C: $\backslash$ Users $\backslash$ LabVIEW-user $\backslash A p p D a t a \backslash L o c a l \backslash T e m p \backslash S w e e p ~ U p$ and Down v5e - Neg to Pos - MF selection and Cryostate added..vi

Last modified on 5/20/2016 at 6:27 PM

Printed on 5/20/2016 at 6:28 PM

Sweep Up and Down v5e - Neg to Pos - MF selection and CryoState added..vi

Magnetic Field Sample \#

Layer \# Cryo State Operator

Comments

Calculates the current from the total number of steps. 
Sweep Up and Down v5e - Neg to Pos - MF selection and CryoState added..vi

Page $2 \amalg-V$ C: $\backslash$ Users $\backslash$ LabVIEW-user $\backslash A p p D a t a \backslash L o c a l \backslash T e m p \backslash S w e e p ~ U p$ and Down v5e - Neg to Pos - MF selection and CryoState added..vi

Last modified on 5/20/2016 at 6:27 PM

Printed on 5/20/2016 at 6:28 PM

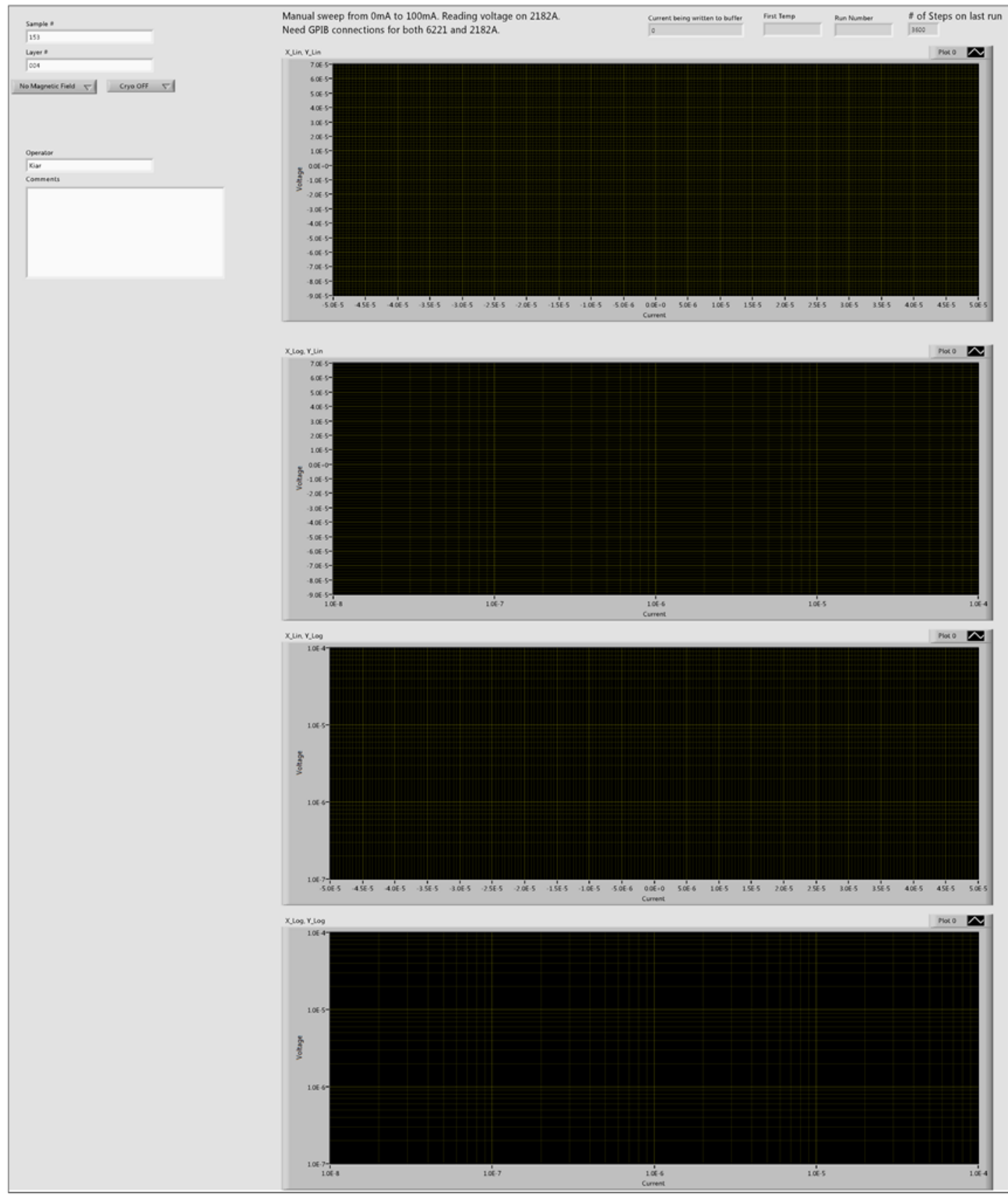


Sweep Up and Down v5e - Neg to Pos - MF selection and CryoState added..vi

C:\Users\LabVIEW-user\AppData\Local\Temp\Sweep Up and Down v5e - Neg to Pos - MF selection and CryoState added..vi

Last modified on 5/20/2016 at 6:27 PM

Printed on 5/20/2016 at 6:28 PM

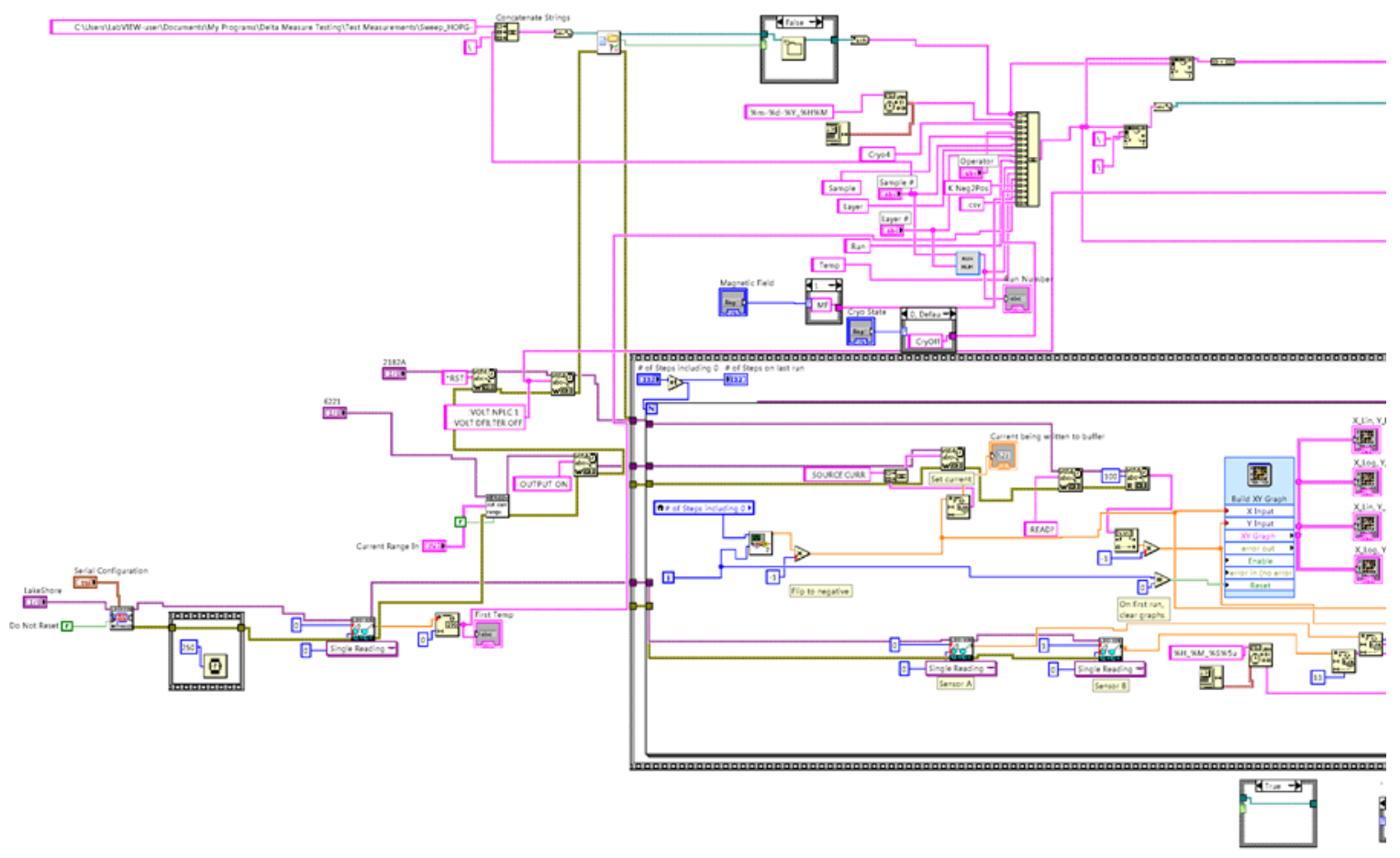

Build XY Graph

Build XY Graph

formats the data displayed on an X-Y Graph. 
Sweep Up and Down v5e - Neg to Pos - MF selection and CryoState added..vi

Page $4 \amalg$

C: $\backslash$ Users $\backslash$ LabVIEW-user $\backslash A p p D a t a \backslash L o c a l \backslash T e m p \backslash S w e e p ~ U p$ and Down v5e - Neg to Pos - MF selection and CryoState added..vi

Last modified on 5/20/2016 at 6:27 PM

Printed on 5/20/2016 at 6:28 PM

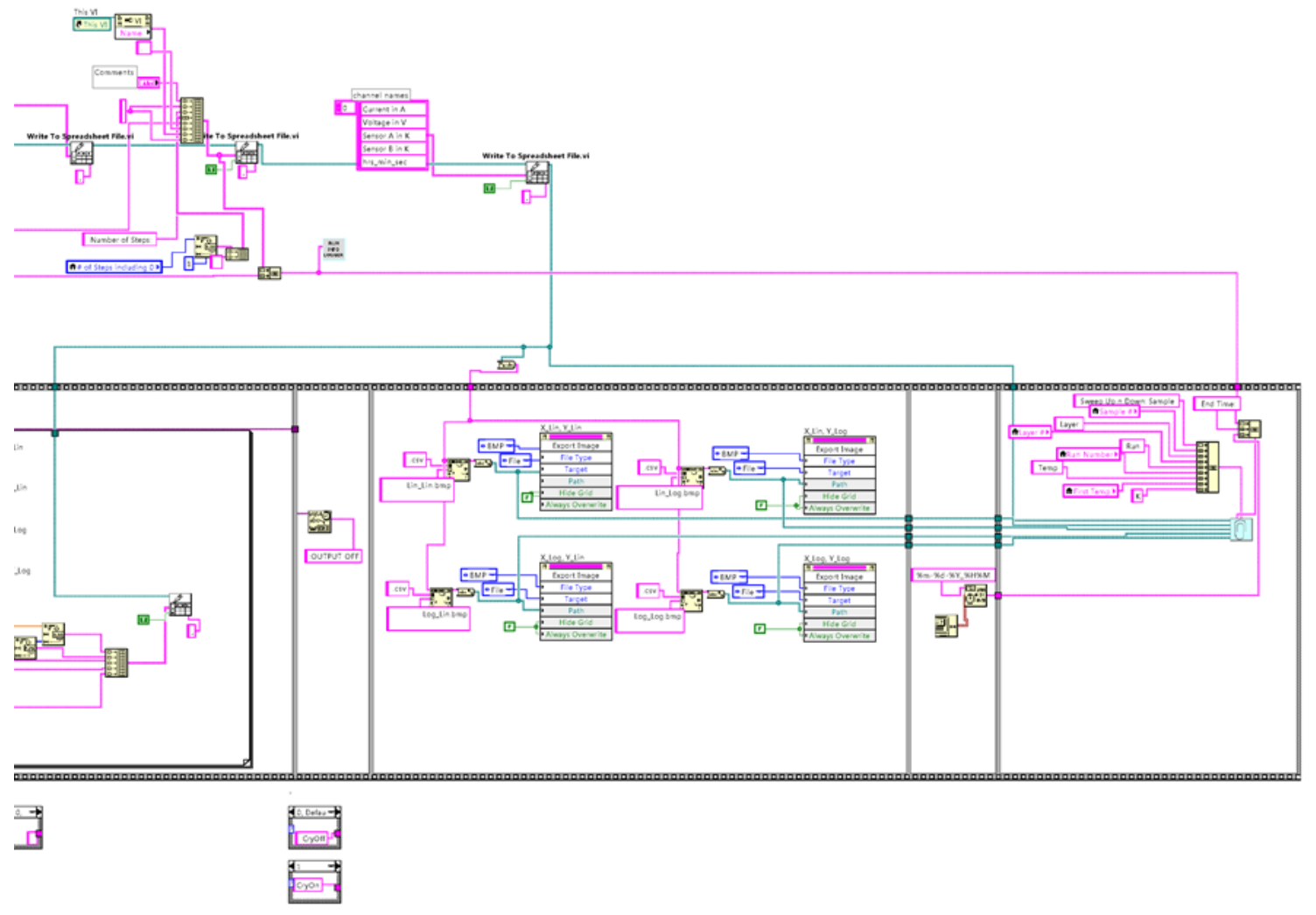


Appendix $50 \quad$ TextMessageGeneral.vi 
TextMessageGeneral.vi

C: $\backslash$ Users $\backslash$ LabVIEW-user $\backslash A p p D a t a \backslash L o c a l \backslash T e m p \backslash T e x t M e s s a g e G e n e r a l . v i$

Last modified on 5/20/2016 at 6:28 PM

Printed on 5/20/2016 at 6:29 PM

TextMessageGeneral.vi

Recipient's Email Address

Extra Email Addresses

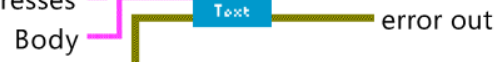

error in (no error)
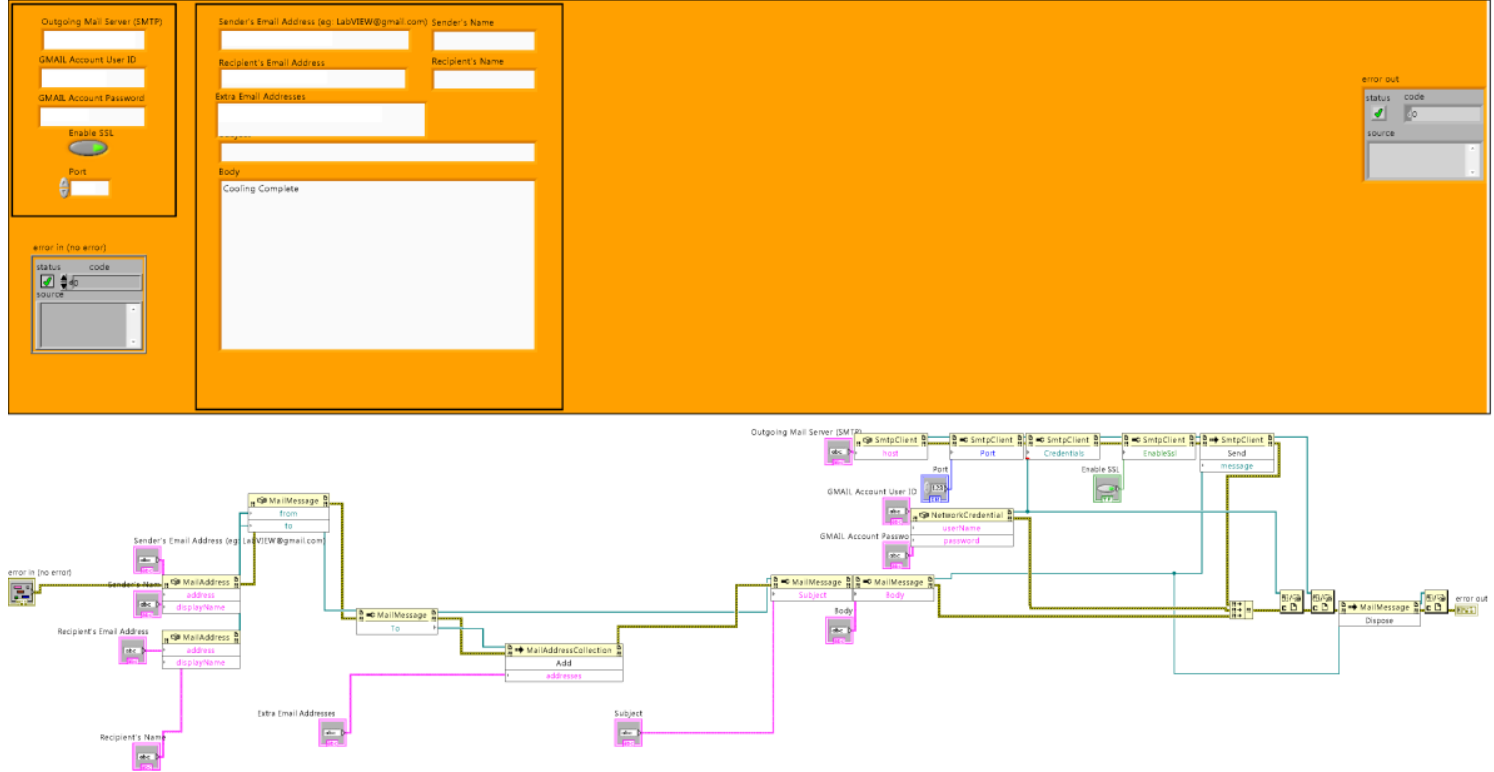
Appendix 51 Voltage reading with DC current always on v4 Compressor

Automatically on and off.vi 
Voltage reading with DC current always on v4 Compressor Automatically on and off.vi

C: $\backslash$ Users $\backslash$ LabVIEW-user $\backslash A p p D a t a \backslash L o c a l \backslash T e m p \backslash V o l t a g e$ reading with $\mathrm{DC}$ current always on $\mathrm{v} 4$

Compressor Automatically on and off.vi

Last modified on 5/20/2016 at 6:29 PM

Printed on 5/20/2016 at 6:30 PM

Voltage reading with DC current always on v4 Compressor Automatically on and off.vi

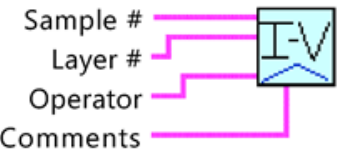


Voltage reading with DC current always on v4 Compressor Automatically on and off.vi C:\Users\LabVIEW-user \AppData\Local\Temp\Voltage reading with DC current always on v4 Compressor Automatically on and off.vi

Last modified on 5/20/2016 at 6:29 PM

Printed on 5/20/2016 at 6:30 PM

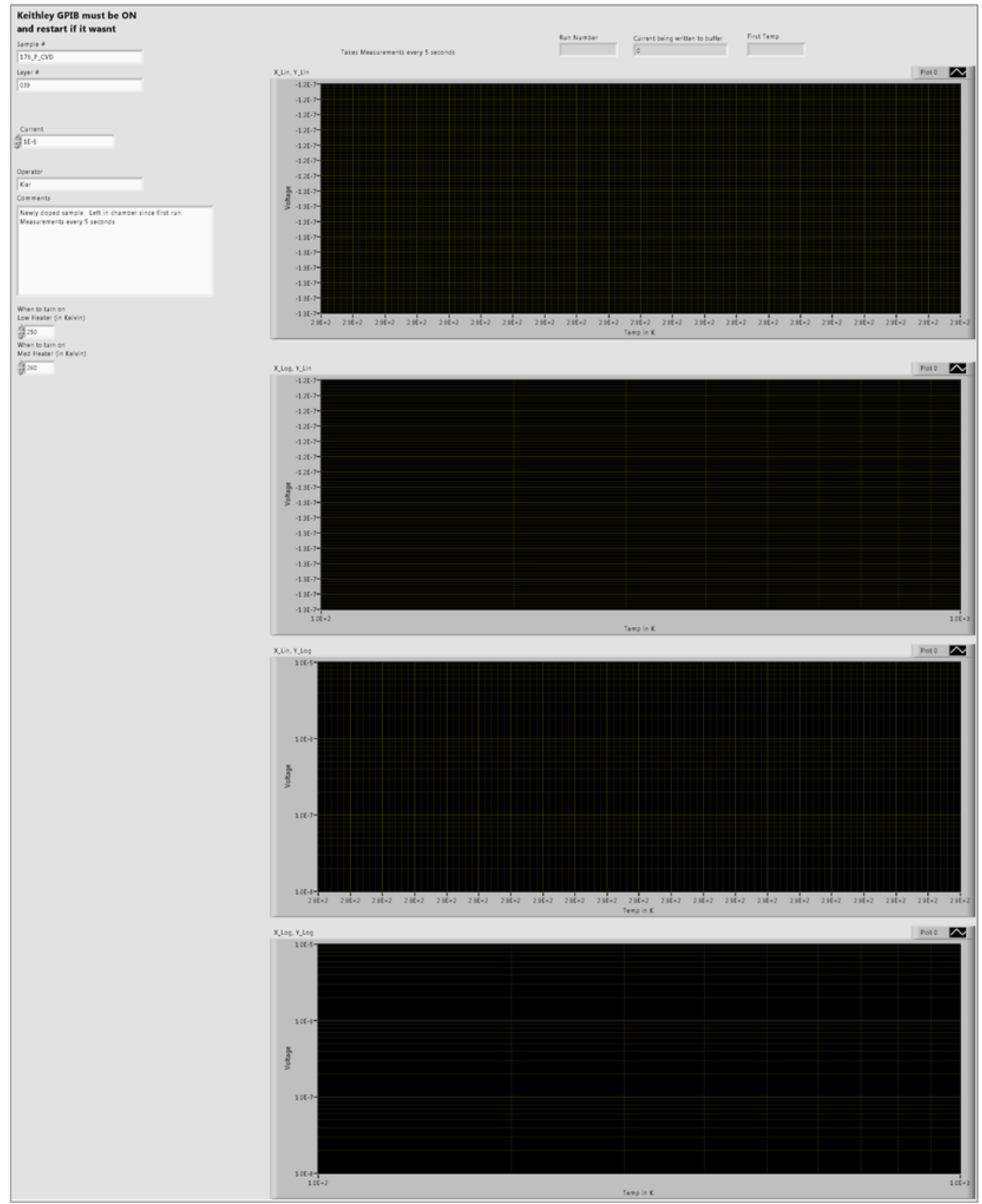


Voltage reading with DC current always on v4 Compressor Automatically on and off.vi

Page $3 \longdiv { - V }$ C:\Users\LabVIEW-user \AppData\Local\Temp\Voltage reading with DC current always on v4 Compressor Automatically on and off.vi Last modified on 5/20/2016 at 6:29 PM Printed on 5/20/2016 at 6:30 PM

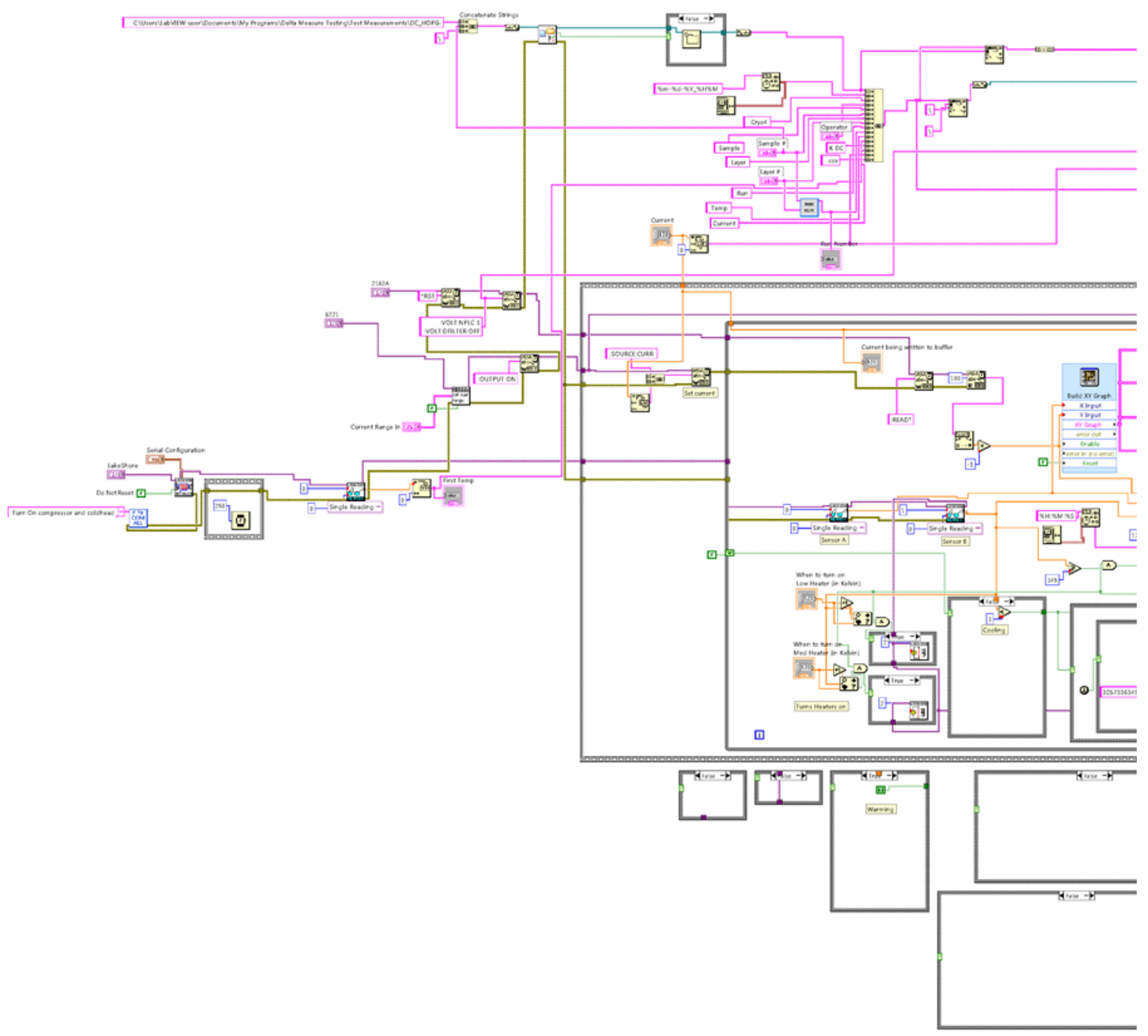

Build XY Graph

Build XY Graph

formats the data displayed on an X-Y Graph. 
Voltage reading with DC current always on v4 Compressor Automatically on and off.vi

Page 4 C:\Users\LabVIEW-user \AppData\Local\Temp\Voltage reading with DC current always on v4 Compressor Automatically on and off.vi Last modified on 5/20/2016 at 6:29 PM

Printed on 5/20/2016 at 6:30 PM
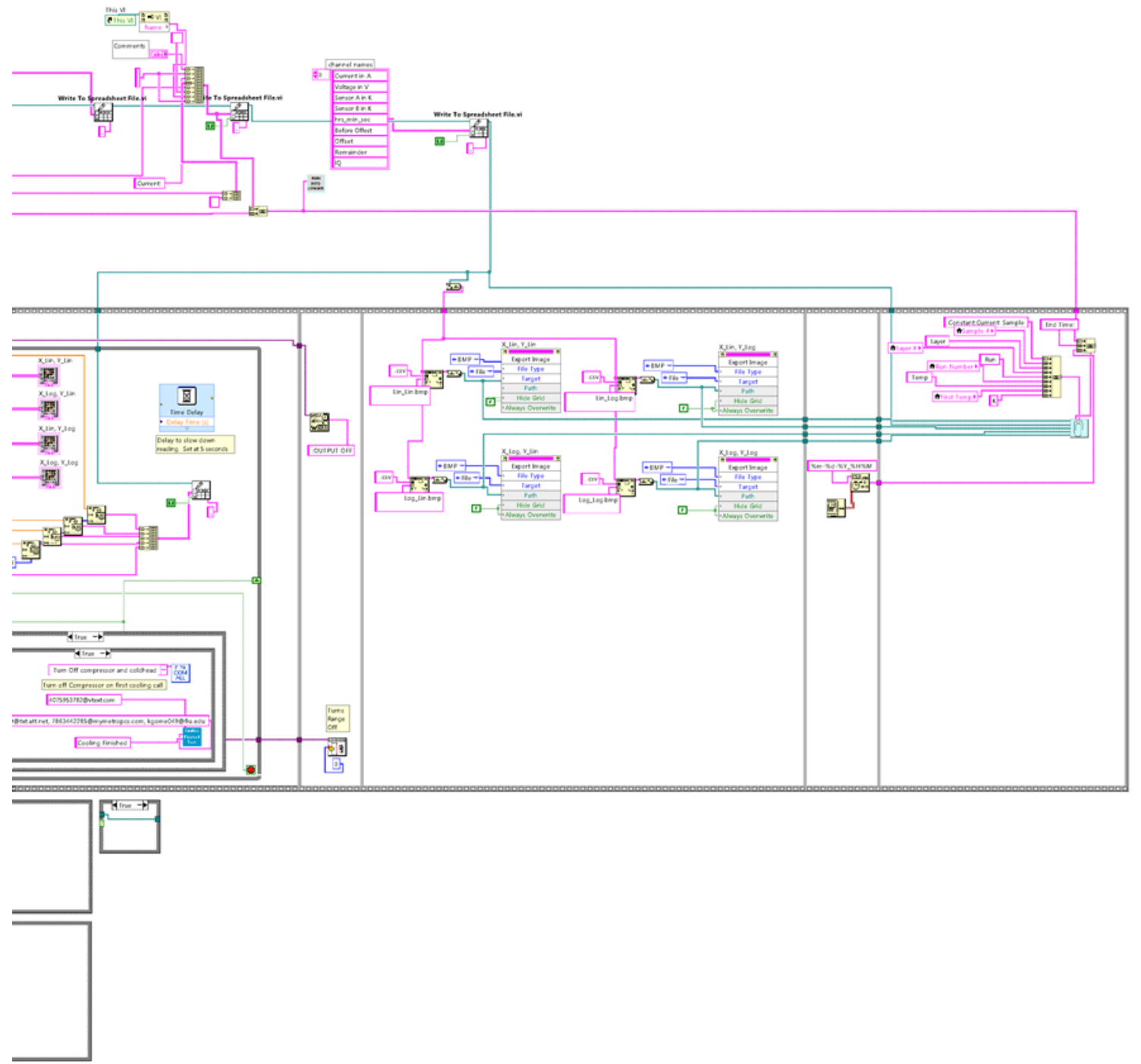
Voltage reading with DC current always on v4 Compressor Automatically on and off.vi

C: $\backslash$ Users $\backslash$ LabVIEW-user $\backslash A p p D a t a \backslash L o c a l \backslash T e m p \backslash V o l t a g e$ reading with DC current always on $v 4$

Compressor Automatically on and off.vi

Last modified on 5/20/2016 at 6:29 PM

Printed on 5/20/2016 at 6:30 PM

\section{区 Time Delay \\ Time Delay \\ Inserts a time delay into the calling VI.}

This Express VI is configured as follows:

Delay Time: $5 \mathrm{~s}$ 
VITA

KIAR HOLLAND

Born, Long Island, New York

2002-2007

Dean's List

Florida International University

Miami, Florida

B.S., Electrical Engineering

Florida International University

Miami, Florida

2005-2009

Nano Bio Sensor G.A. and G.R.A.

Florida International University

Miami, Florida

M.S., Electrical Engineering

Florida International University

Miami, Florida

2013-2014

McKnight Doctoral Fellowship

2010-2016

High Temperature Superconductors G.A. and G.R.A.

Florida International University

Miami, Florida

Doctoral Candidate

Florida International University

Miami, Florida

\section{PUBLICATIONS}

Larkins, G., Vlasov, Y. and Holland, K. (2015) Evidence of Superconductivity in Doped Graphite and Graphene. Superconductor Science and Technology, Volume 29, Number 1. 